Fall 2016

\title{
2016 Miracle Yearbook
}

Cedarville University

Follow this and additional works at: https://digitalcommons.cedarville.edu/yearbooks

Part of the Higher Education Commons, Organizational Communication Commons, and the Public Relations and Advertising Commons

\section{Recommended Citation}

Cedarville University, "2016 Miracle Yearbook" (2016). Yearbooks. 97.

https://digitalcommons.cedarville.edu/yearbooks/97

This Book is brought to you for free and open access by DigitalCommons@Cedarville, a service of the Centennial Library. It has been accepted for inclusion in Yearbooks by an authorized administrator of DigitalCommons@Cedarville. For more information, please contact digitalcommons@cedarville.edu. 


\section{Miracle}

Yearbook

2016 




\section{Cedarville Miracle Yearbook 2016}

$\begin{array}{llll}03 \text { Dedication } & 26 \text { Junior Senior } & 50 \text { Soccer } & 72 \text { Academic Teams } \\ 06 \text { Getting Started } & 29 \text { SoFresh } & 52 \text { Cross Country } & 74 \text { Trustees } \\ 07 \text { Fall Bible Conference } & 30 \text { Elliv } & 54 \text { Volleyball } & 76 \text { Faculty and Staff } \\ 08 \text { Homecoming } & 32 \text { Commencement } & 55 \text { Cheerleaders } & 81 \text { Freshmen } \\ 10 \text { Father of The Bride } & 38 \text { Alt Night } & 56 \text { Basketball } & 92 \text { Sophomores } \\ 14 \text { Moonlight Madness } & 40 \text { Org Wars } & 58 \text { Tennis } & 98 \text { Juniors } \\ 16 \text { Campus Christmas } & 42 \text { Chapel } & 60 \text { Track } & 103 \text { Seniors } \\ 18 \text { Mission Conference } & 43 \text { SGA } & 62 \text { Baseball and Softball } & 116 \text { Yearbook Staff } \\ 20 \text { Cyrano de Bergerac } & 44 \text { Concerts } & 64 \text { Golf and Club Sports } & 118 \text { Colophon } \\ 24 \text { Wit } & 46 \text { Global Outreach } & 66 \text { Orgs } & 119 \text { Letter from Editor }\end{array}$




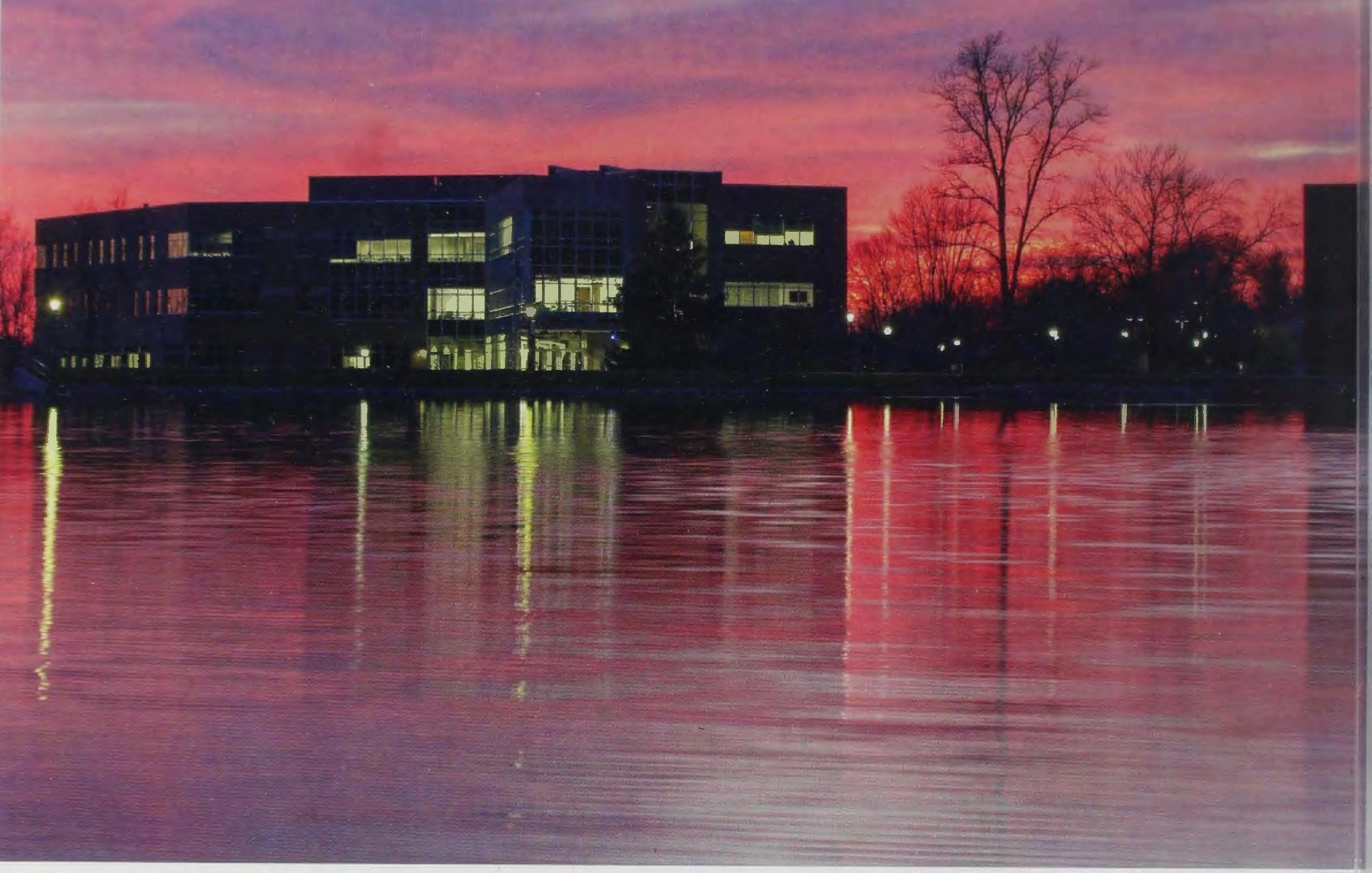

Here we watch as the sun goes down over the Western horizon day after day, and again as it rises in the East. Here we chase adventure like we were made for it, hiking and climbing and seeing all creation, near and far. Here we get late-night snacks from the gas station, and ice cream across the street. Here we shout songs and share tears and devote time to another like no one else before. Here we open doors and share umbrellas and laugh with strangers. These and many more are routine to us. Cedarville has given us a home and a family, and here we have made new traditions that will be remembered for years to come. It is these traditions that make our culture, these routines that we share with all those who have come before us and with those that follow - we build an ever-growing, ever-learning family of timeless culture, sharing our loves and passions and knowledge and growing together forever, seeking the light of Jesus Christ and sharing that light with those around us, near and far.

Even so, it is not us or our words or our works that will last, but the light and the works and the Word of our Father in Heaven, as it is written,

"The grass withers, the flower fades, but the word of our God will stand forever." 

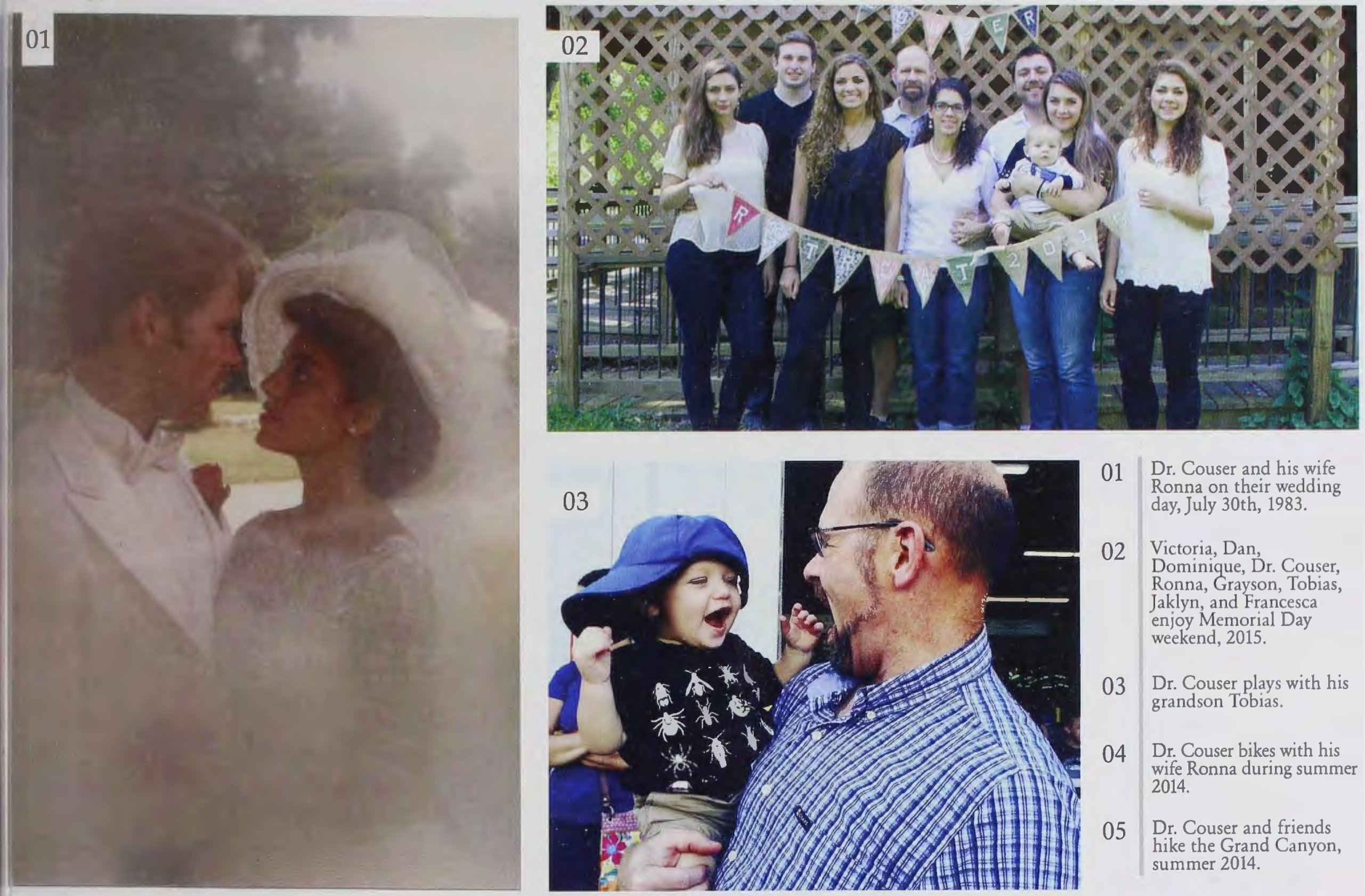

01 Dr. Couser and his wife Ronna on their wedding day, July 30 th, 1983.

02 Victoria, Dan

Dominique, Dr. Couser, Ronna, Grayson, Tobias, Jaklyn, and Francesca enjoy Memorial Day weekend, 2015.

03 Dr. Couser plays with his grandson Tobias.

04 Dr. Couser bikes with his wife Ronna during summe 2014.

05 Dr. Couser and friends hike the Grand Canyon, summer 2014.
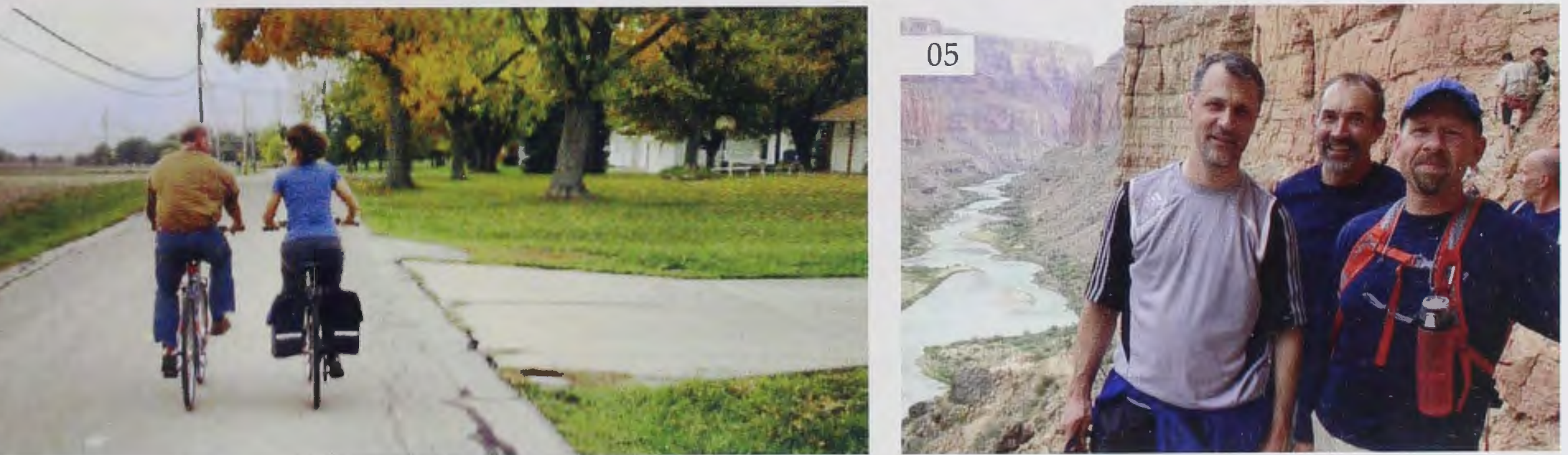

\section{Dedication}

Greg Couser, Senior Professor of Bible and Greek, is a blessing and a providence to the

Cedarville community; one cannot cross paths with this esteemed and loved professor without being encouraged, growing in wisdom, and witnessing the true joy of the Lord in a committed follower of Jesus Christ. Dr. Couser is a loving husband of over thirty years to Ronna Couser, father to four daughters, Jaklyn, Francesca, Victoria, and Dominique, grandfather of two, and confers his loving and caring person also to his teaching at Cedarville University. He has a gentle and fatherly temperament, and his love is for spending time with others in service and growth. His dedication to wisdom and a knowledge of God are evidenced in his love for reading, his commitment to learning, and his desire to engage and encourage others toward a mature relationship with God. To this end, Dr. Couser ministers in the community by teaching and serving as an elder at Emmanuel Baptist Church, by mentoring students and couples on campus, and by dedicating time to various organizations on campus. Cedarville University would be at a great loss without the ministry of Dr. Greg Couser. 


\section{fall}

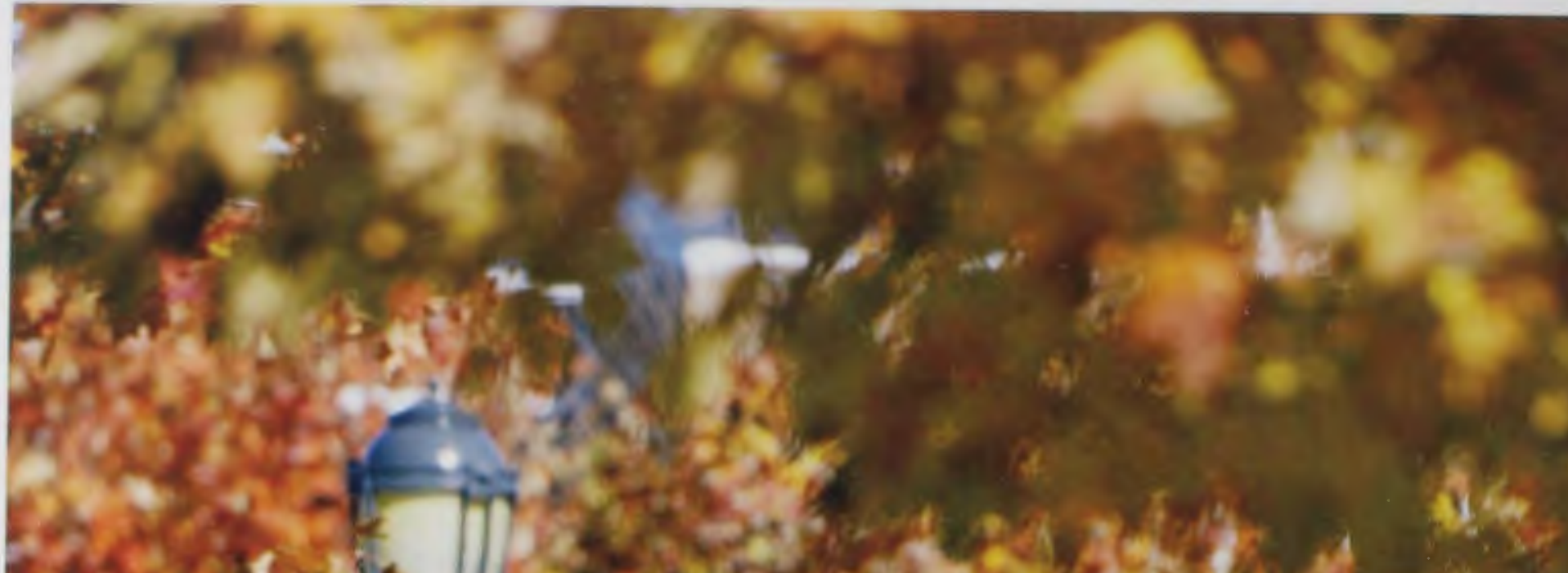

(2)

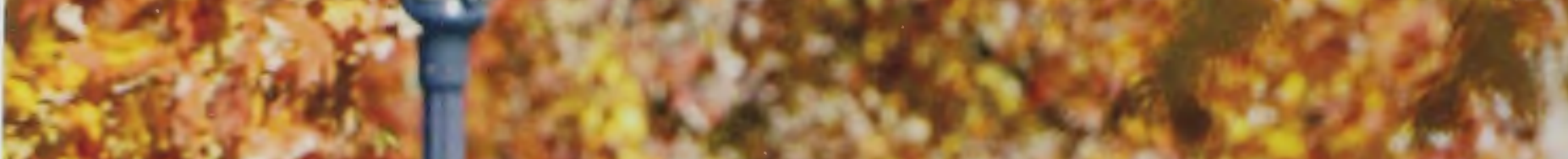

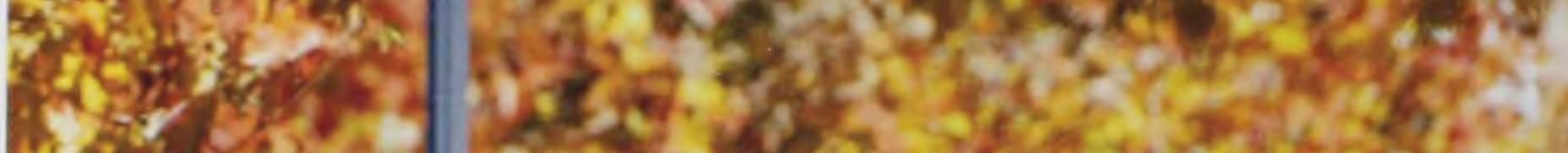

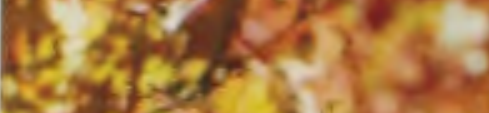

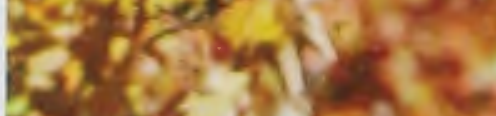

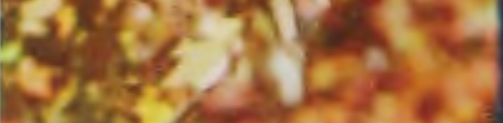

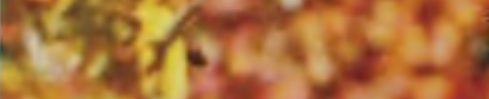

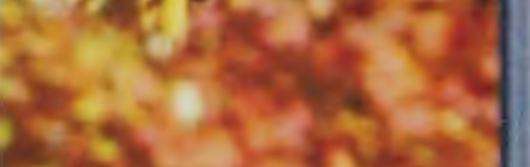

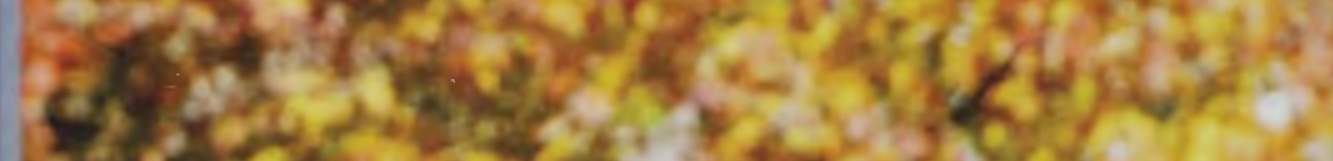

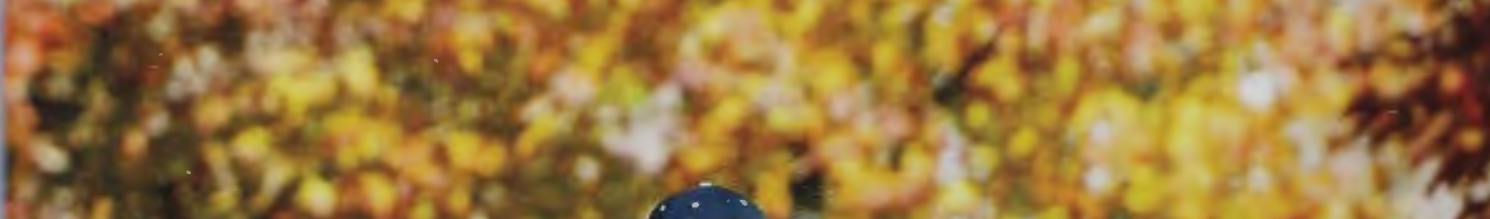

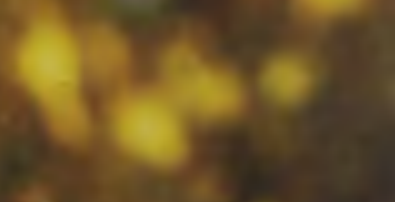
5 $=11$

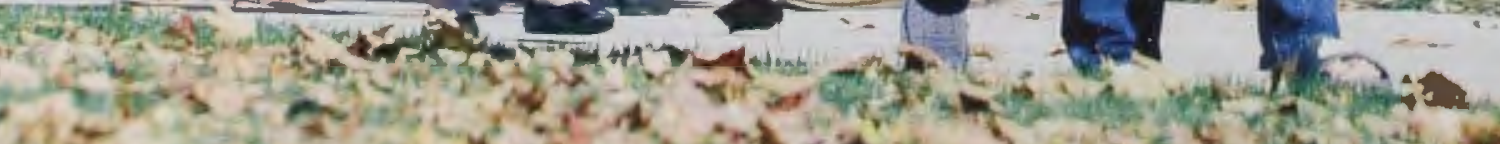

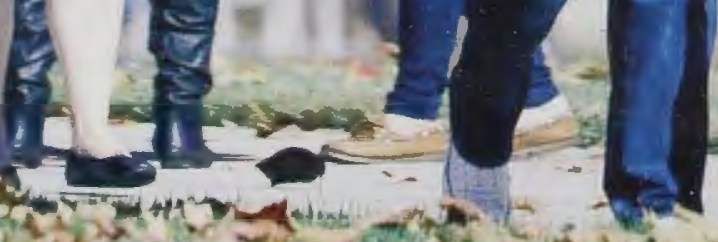

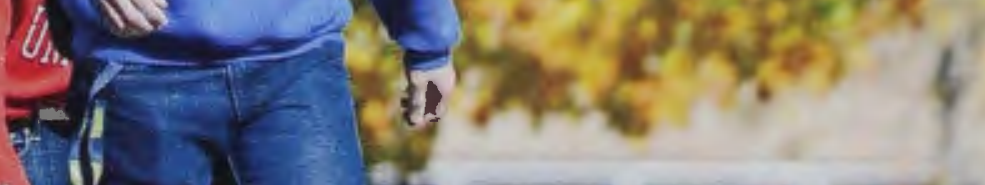

(1)

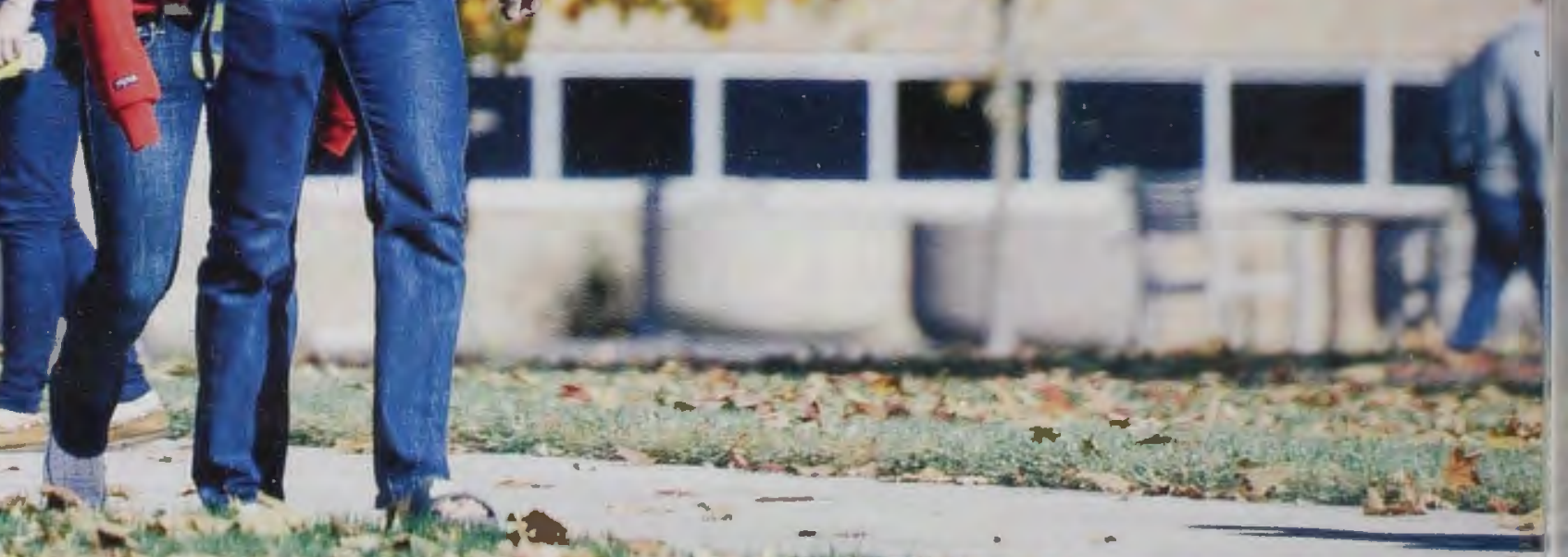

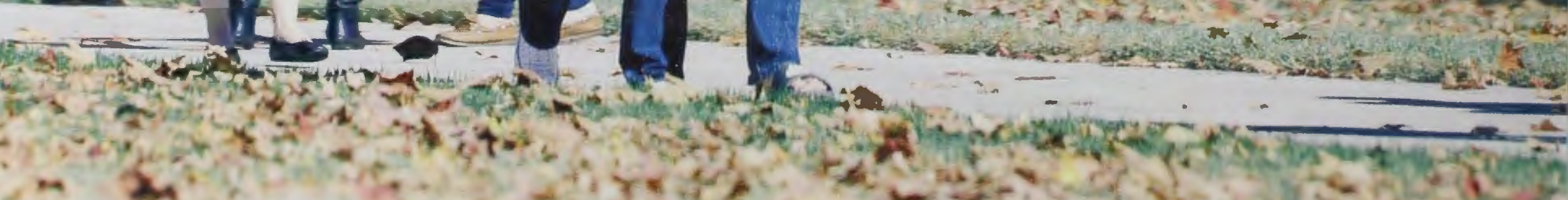
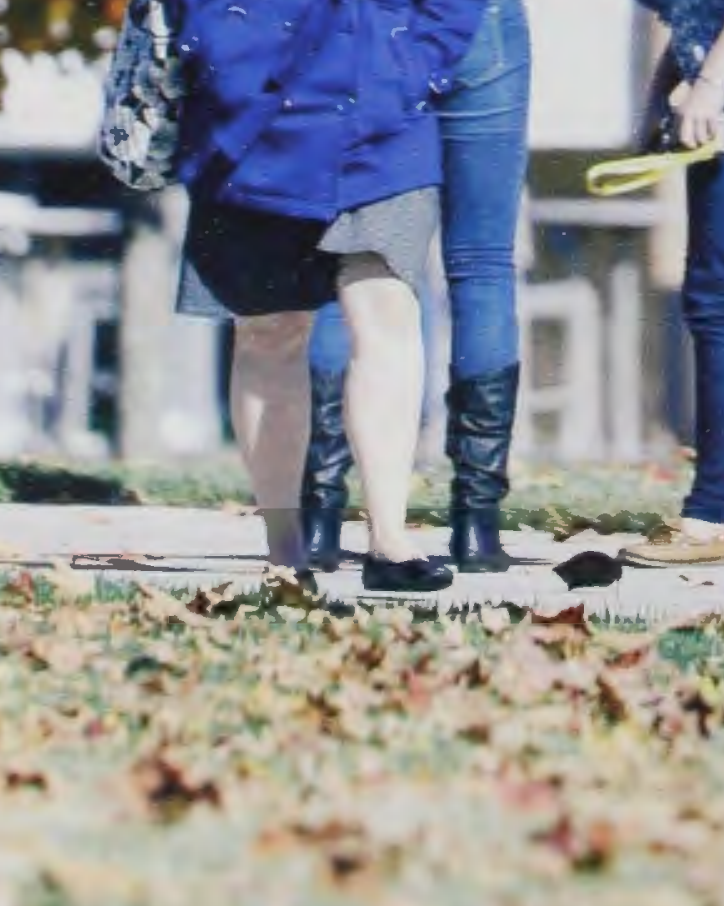

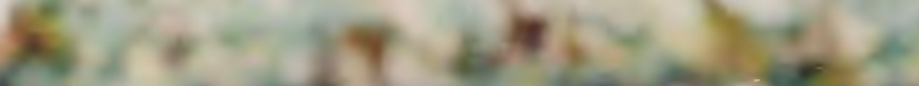



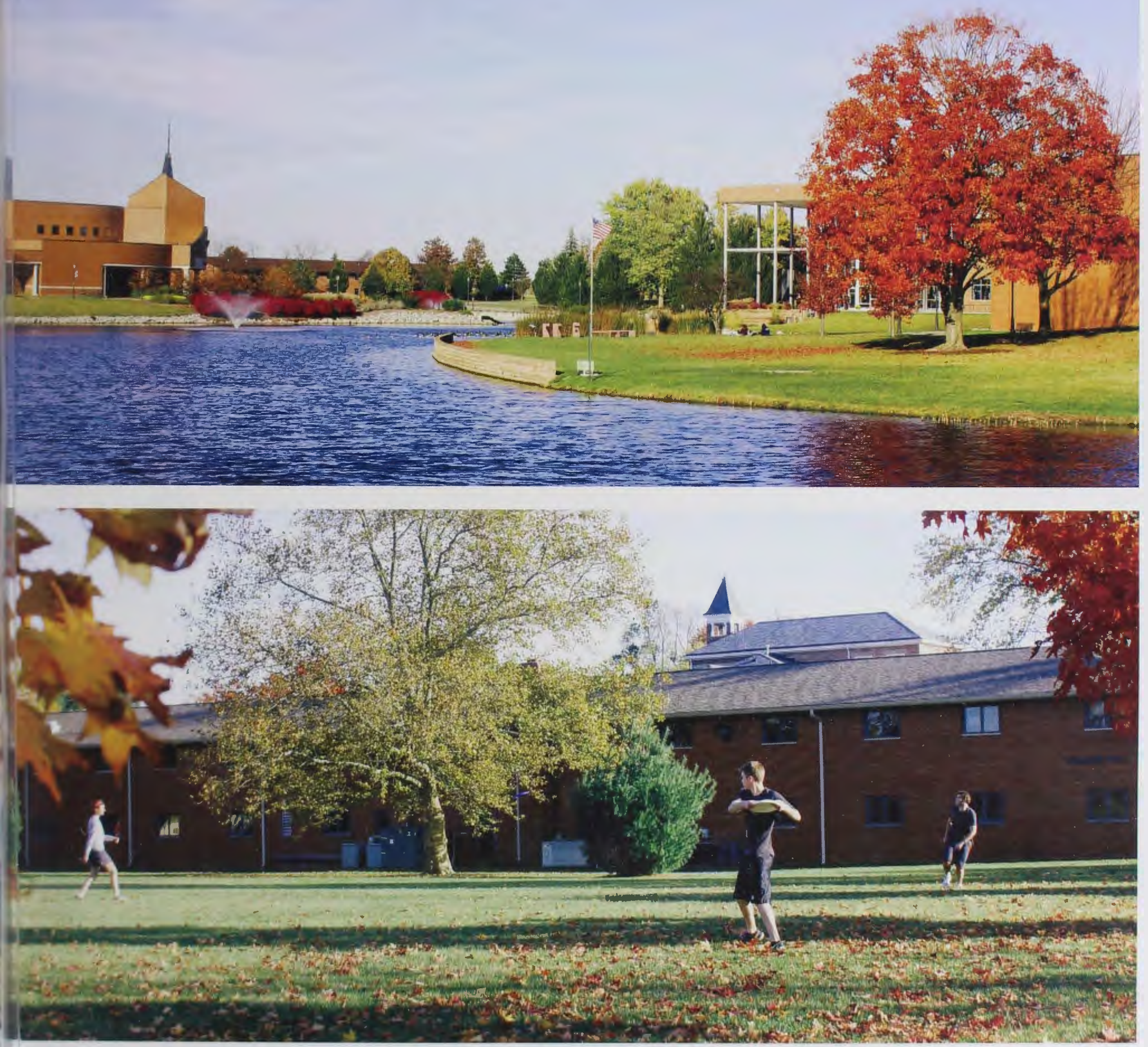

"I was drinking in the surroundings: air so crisp you could snap it with your fingers and greens in every lush shade imaginable offset by autumnal flashes of red and yellow." 

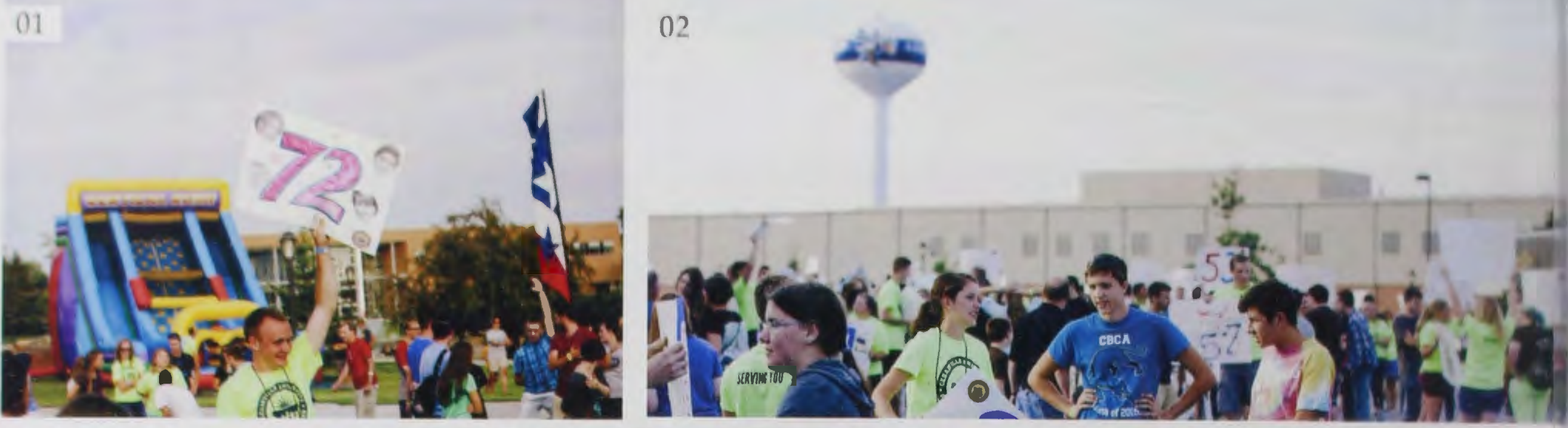

\begin{tabular}{l|l}
01 & $\begin{array}{l}\text { STING Leaders reach } \\
\text { out to freshmen arriv- } \\
\text { ing on campus. }\end{array}$ \\
02 & $\begin{array}{l}\text { STING Leaders prepare } \\
\text { for their small groups } \\
\text { to arrive. } \\
\text { Zach Horst gets ready } \\
\text { to unpack a new stu- } \\
\text { dent's van. }\end{array}$ \\
04 & $\begin{array}{l}\text { Scott Stephens greets } \\
\text { families moving stu- } \\
\text { dents into Lawlor Hall. } \\
\text { New and old students }\end{array}$ \\
05
\end{tabular}
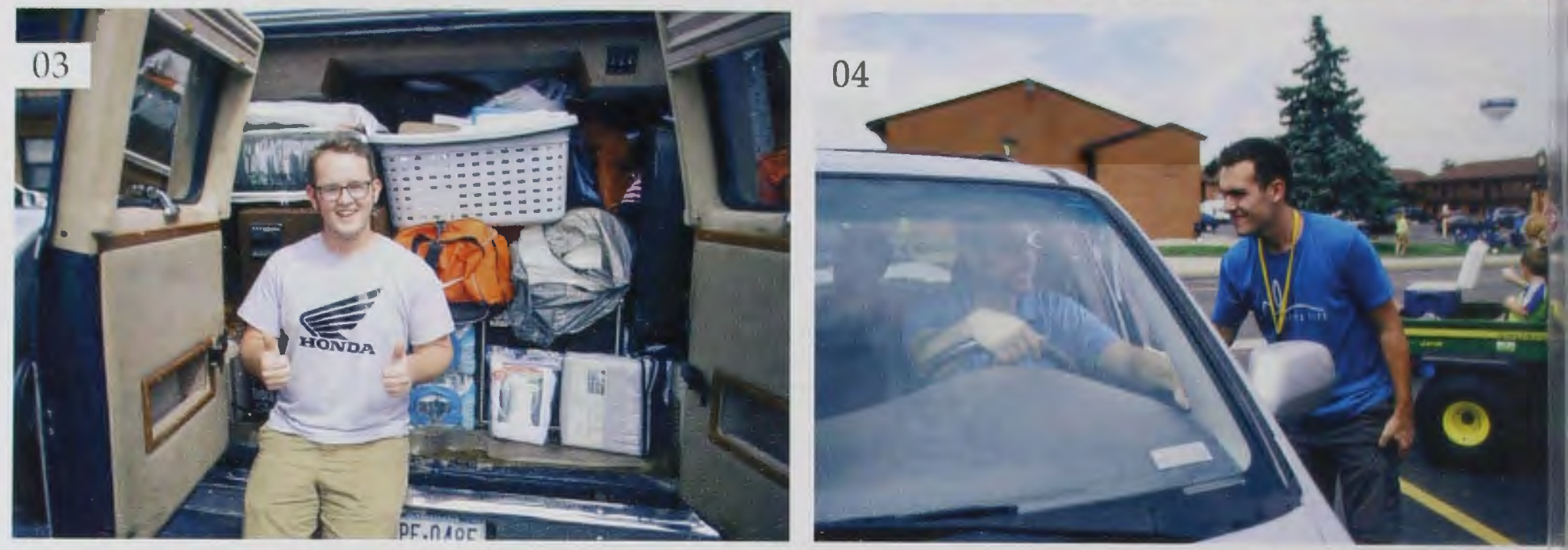
participate in the Jacket

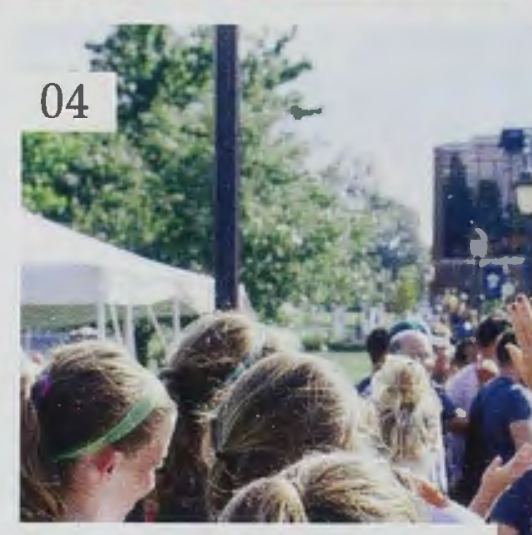

"Getting started showed me how much of a community Cedarville is. There were so many people helping, it felt like home."
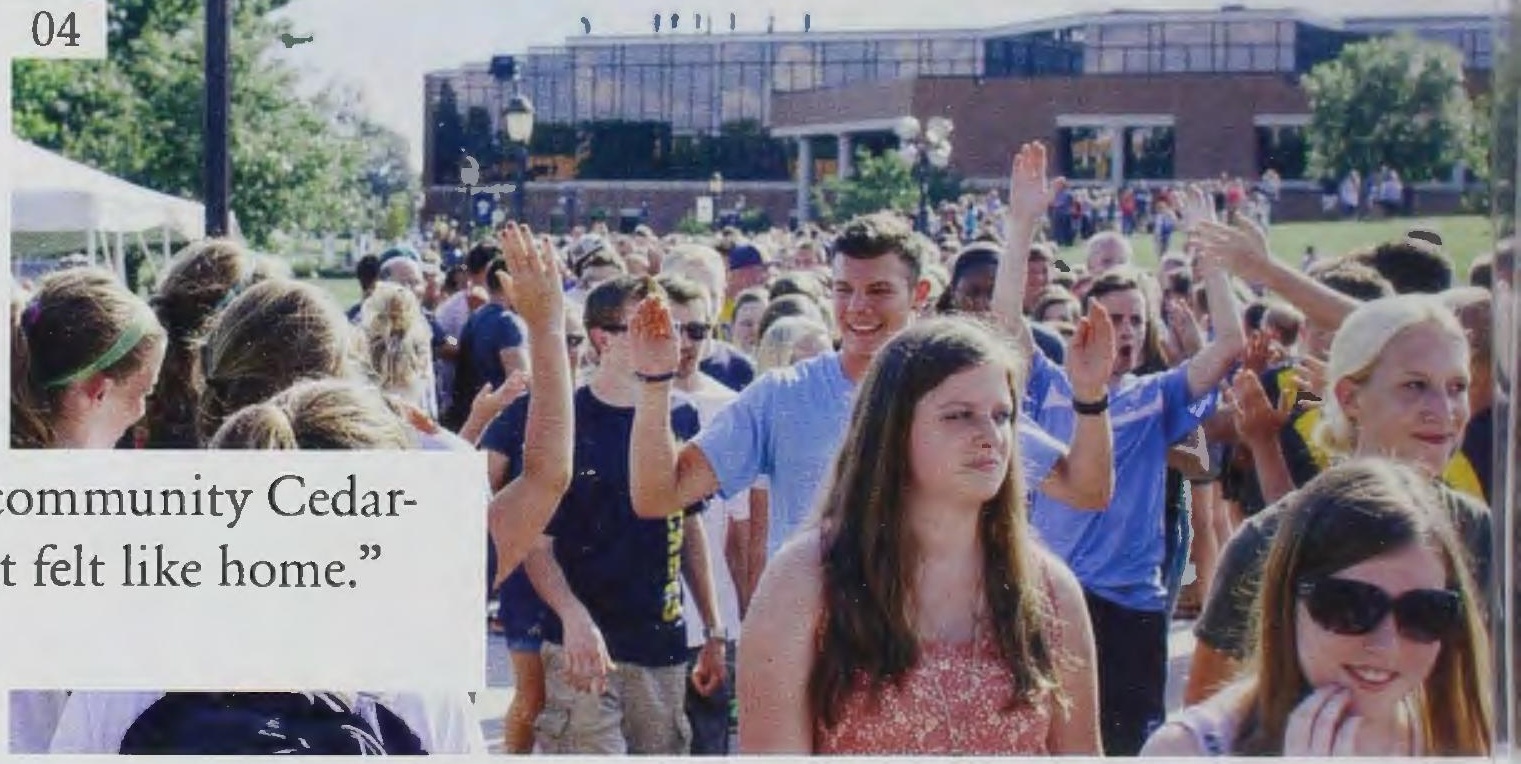

\section{Getting Started}

The Cedarville campus is bustling and crowded with people and the energy of something new. Our family comes together here and now to grow and learn and move forward in life and unity. It is during Getting Started weekend that life-long friends are found, community is sown, and a home is made. No matter how much time is spent on Cedarville's campus, students always remember what happened their first few days here: meeting Dr. White, getting their key, bonding with their roommate, meeting the faculty they will work with for the next several years, and preparing themselves for the life to come. They do not know how important these moments are to the rest of their lives. 

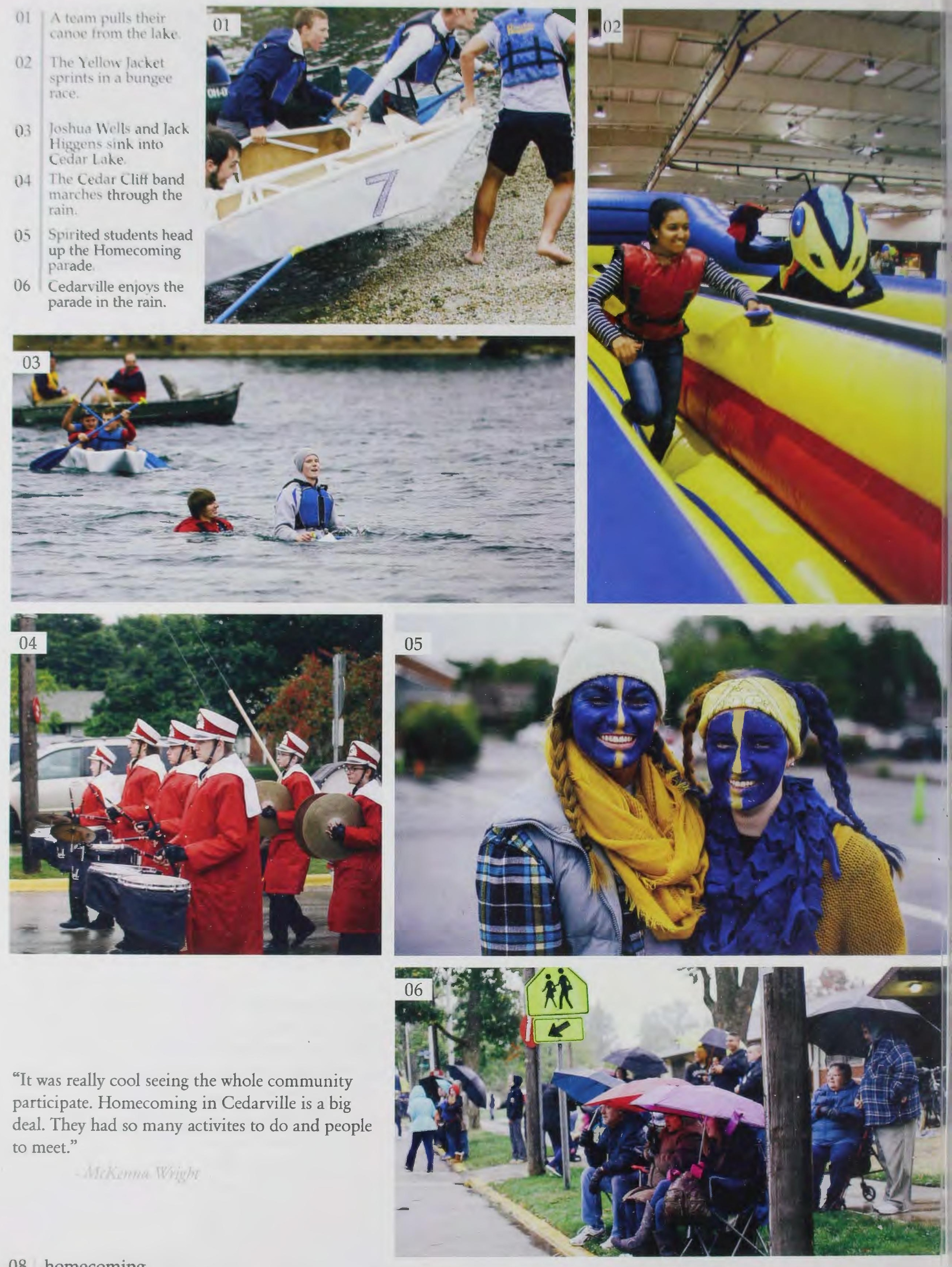

"It was really cool seeing the whole community participate. Homecoming in Cedarville is a big deal. They had so many activites to do and people to meet." 


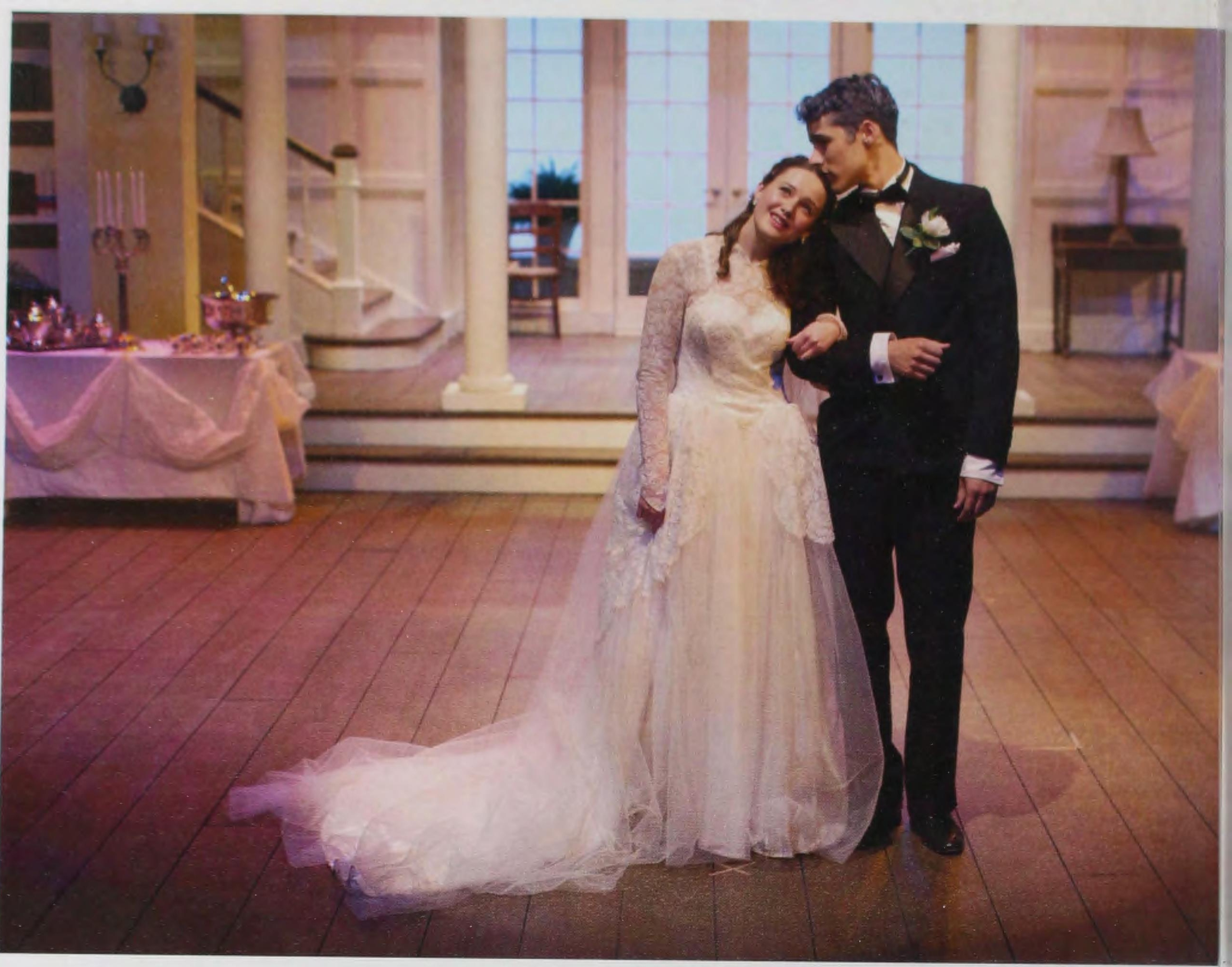

\section{Fall Play}

Presented homecoming weekend by a cast of 14 to an audience of alumni, families, and students, was the Cedarville theatre department's fall production, Father of the Bride. Set in the ' 50 s, it was a comedic tale of the chaotic process of wedding planning and its effects of the family, with the spotlight on the father, frantically juggling the demands of the wedding and struggling with the growth of his little girl. Cedarville University's theatre department cherishes the privilege to share their gifts, talents, and passions with their community and their audience, providing entertainment for others and truly connecting people with their art. 


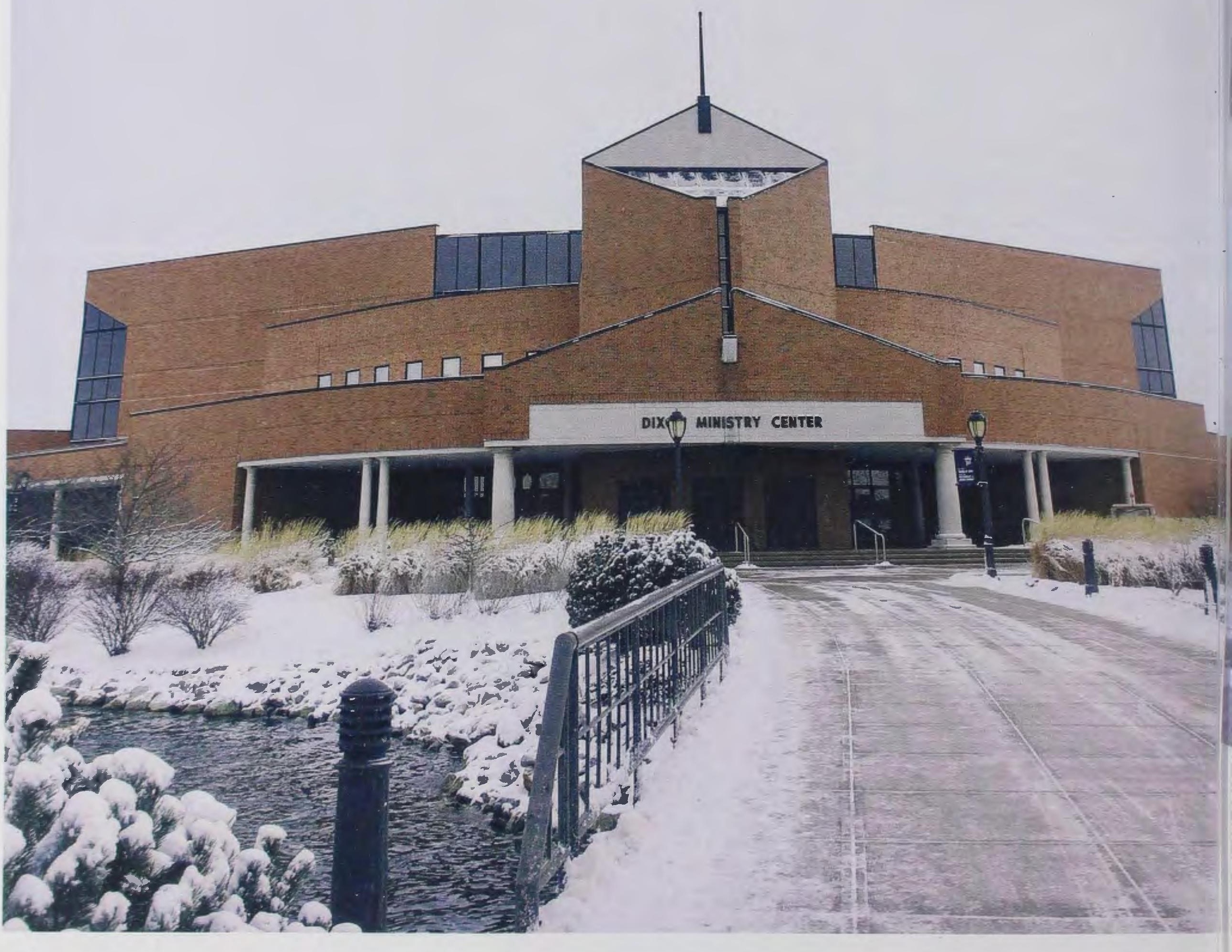




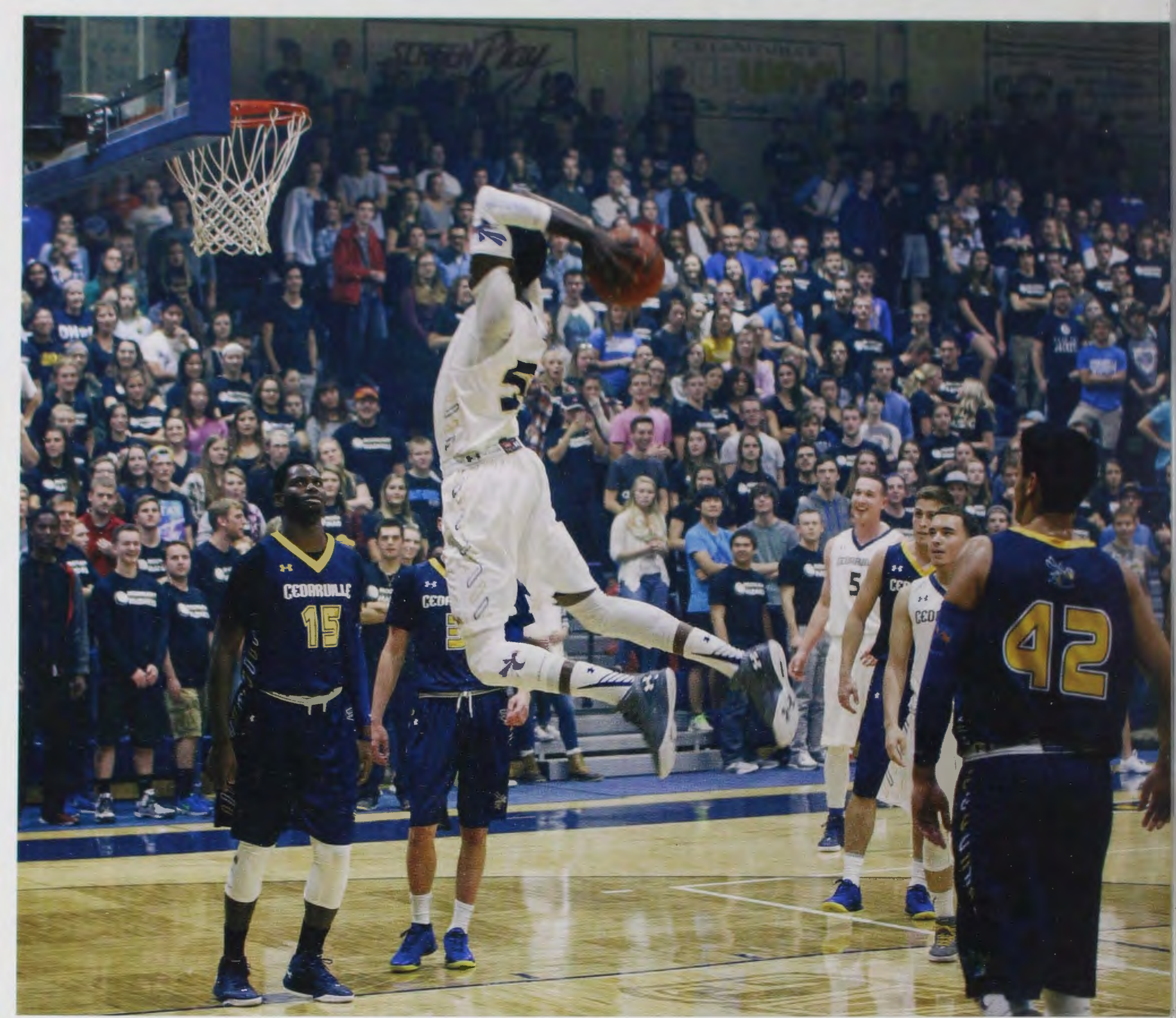

\section{Moonlight Madness}

Moonlight Madness kicks off the Yellow Jackets varsity basketball season each year. The basketball teams go all out in a night of excitement, lights, action, and noise to introduce the year's men's and women's varsity teams. The community gets hyped for the season with scrimmages, a showdown game

between the students and faculty, audience contests, the Yellow Jacket Pep Band, and the beloved dunk contest to top it all off. 


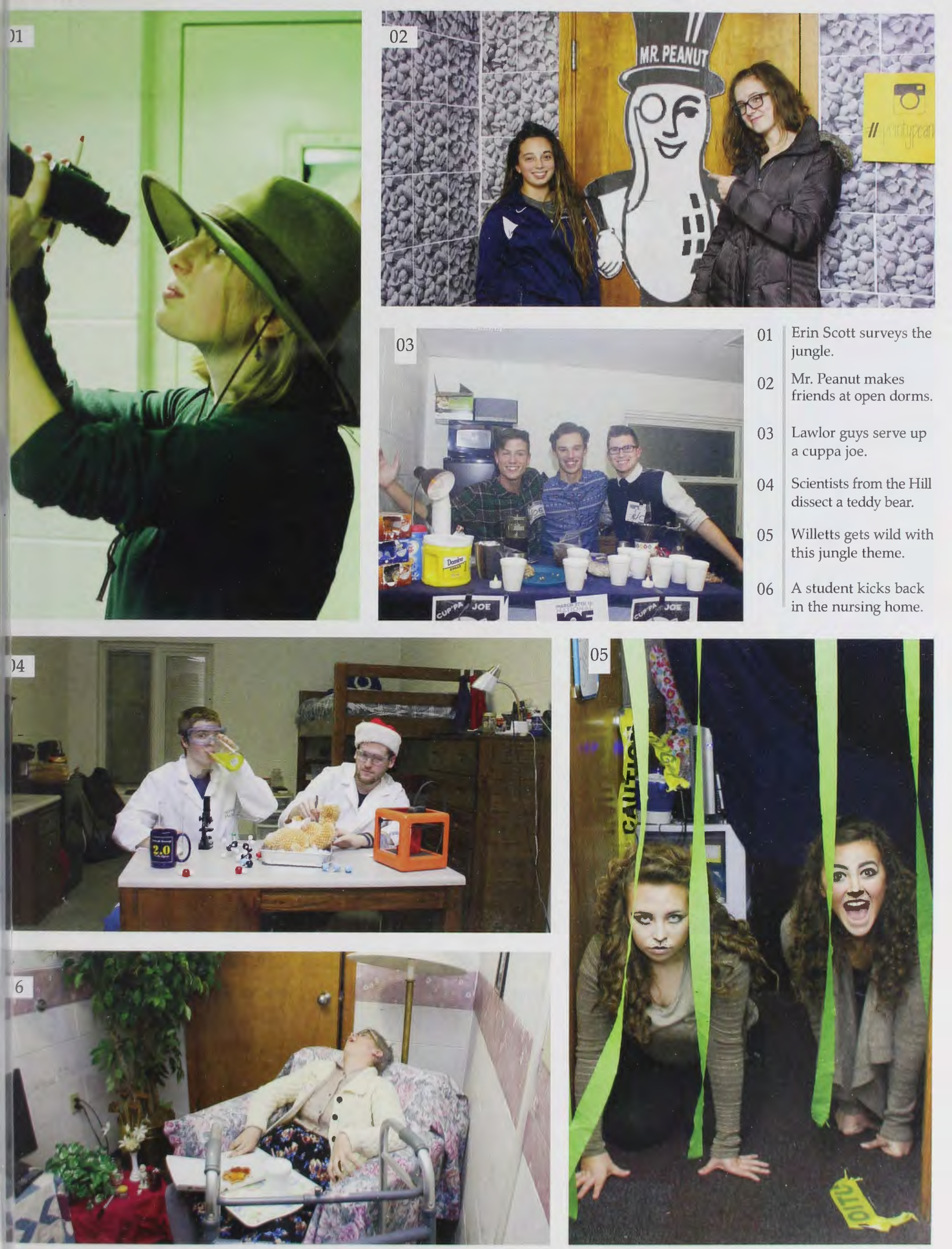




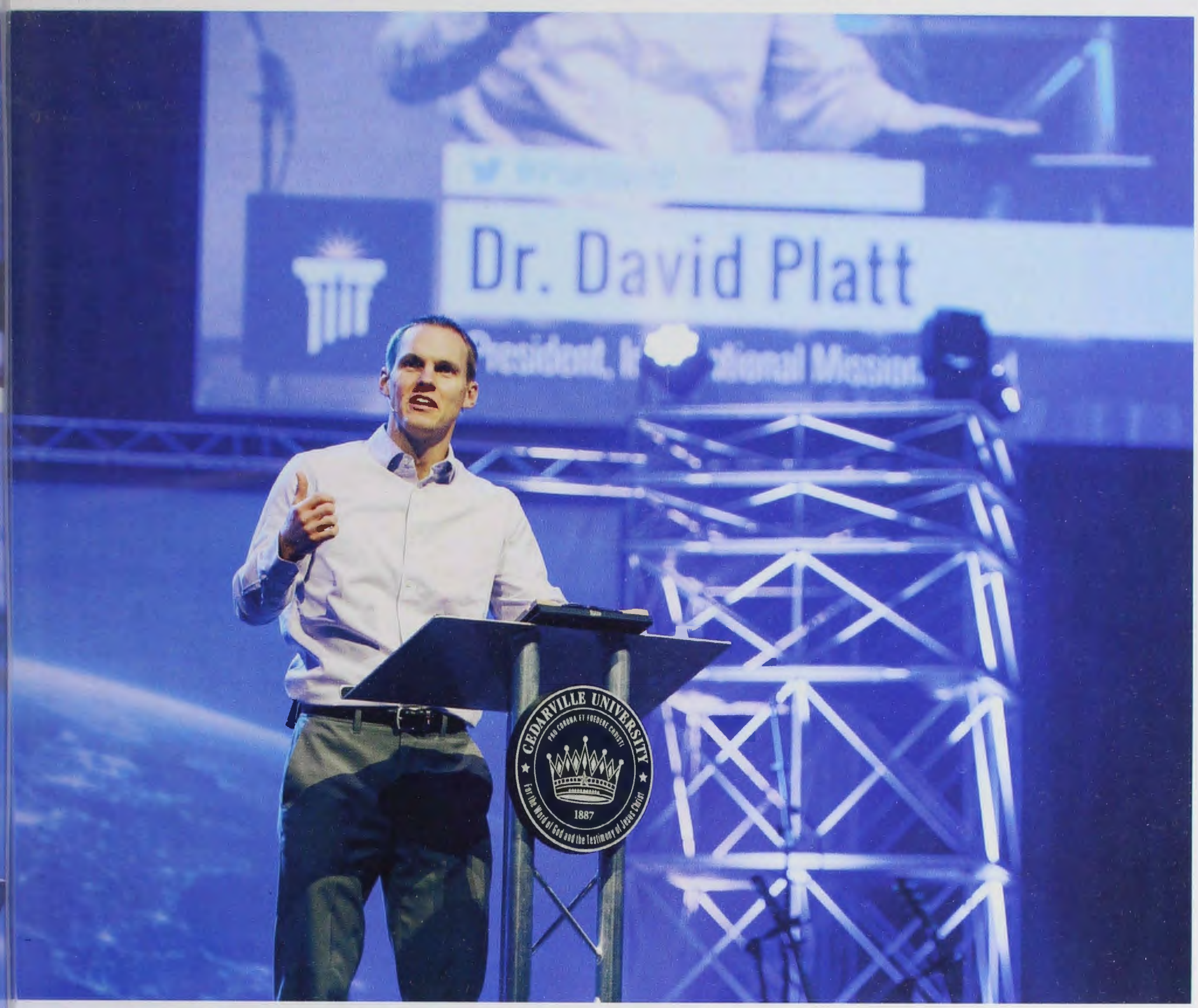

\section{Mission Conference}

The annual Mission

Conference brings the

Cedarville community

together as a reminder of the great, deep need for the relief, peace, and love of God in the world. Missionaries and organizations came to speak to students about their work and the opportunities they have to bring the gospel and God's love to the nations. David Platt, president of the International Mission Board, pleaded the great urgency for Christians to share the gospel with the desperate world that needs it and reminded those listening that there are some in the world who have never heard about the great love and care the Lord has for them. Harry Gebert, Rob Turner, and Scott Dunford also offered wisdom and exhortation on the character of mission-minded Christians and the call to God's mission for all Christians. 


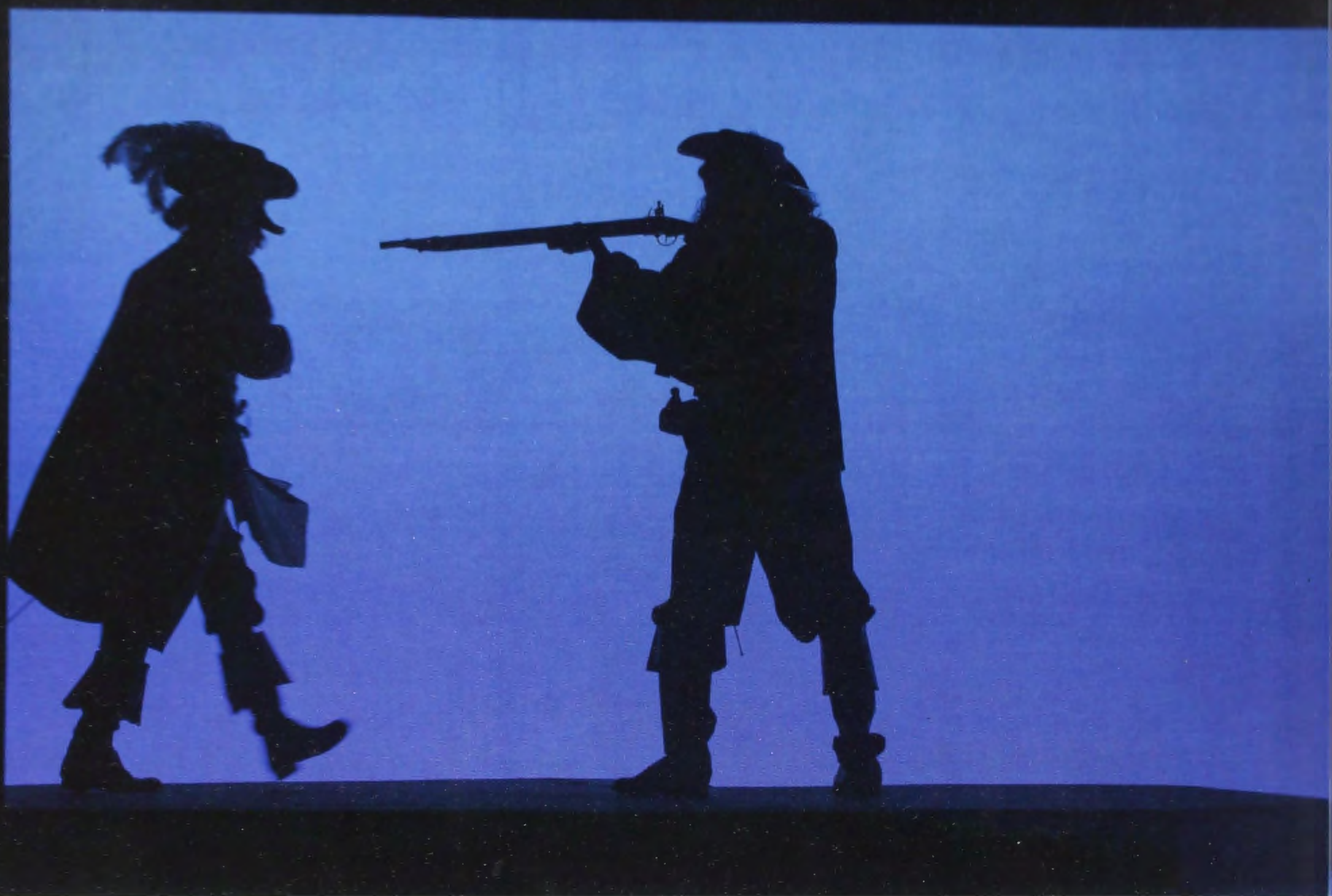

\section{Cyrano de Bergerac}

Cedarville's Theatre

Department performed

Cyrano de Bergerac, a

romantic story, during

the week of Valentine's

Day. Taking Place in the

17th Century, Cyrano,

a big-hearted and big-

nosed man attmepts to win over his distant cousin and love, Rox- love. Throughout the anne. Cyrano, played by play, Cyrano's other David Widder-Varhegyi portrays the struggle between Cyrano's big heart and his self-doubt due to his large nose. His large nose is his biggest obstacle and gets in the way of his search for desireable traits are

shown, like his dueling and musician-like skills, but he still doubts his appearance, which makes him believe that he is unlovable by any woman. 


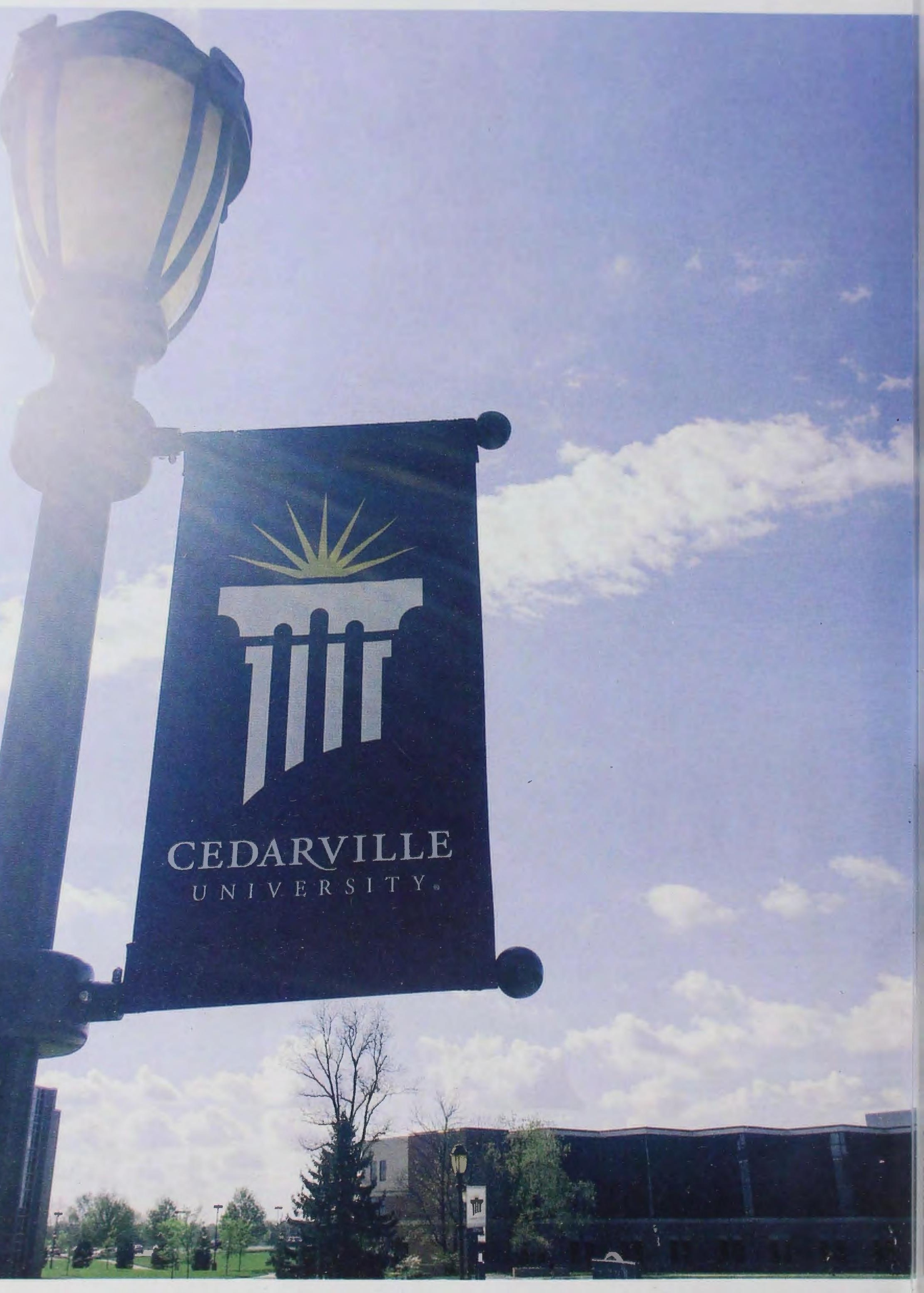



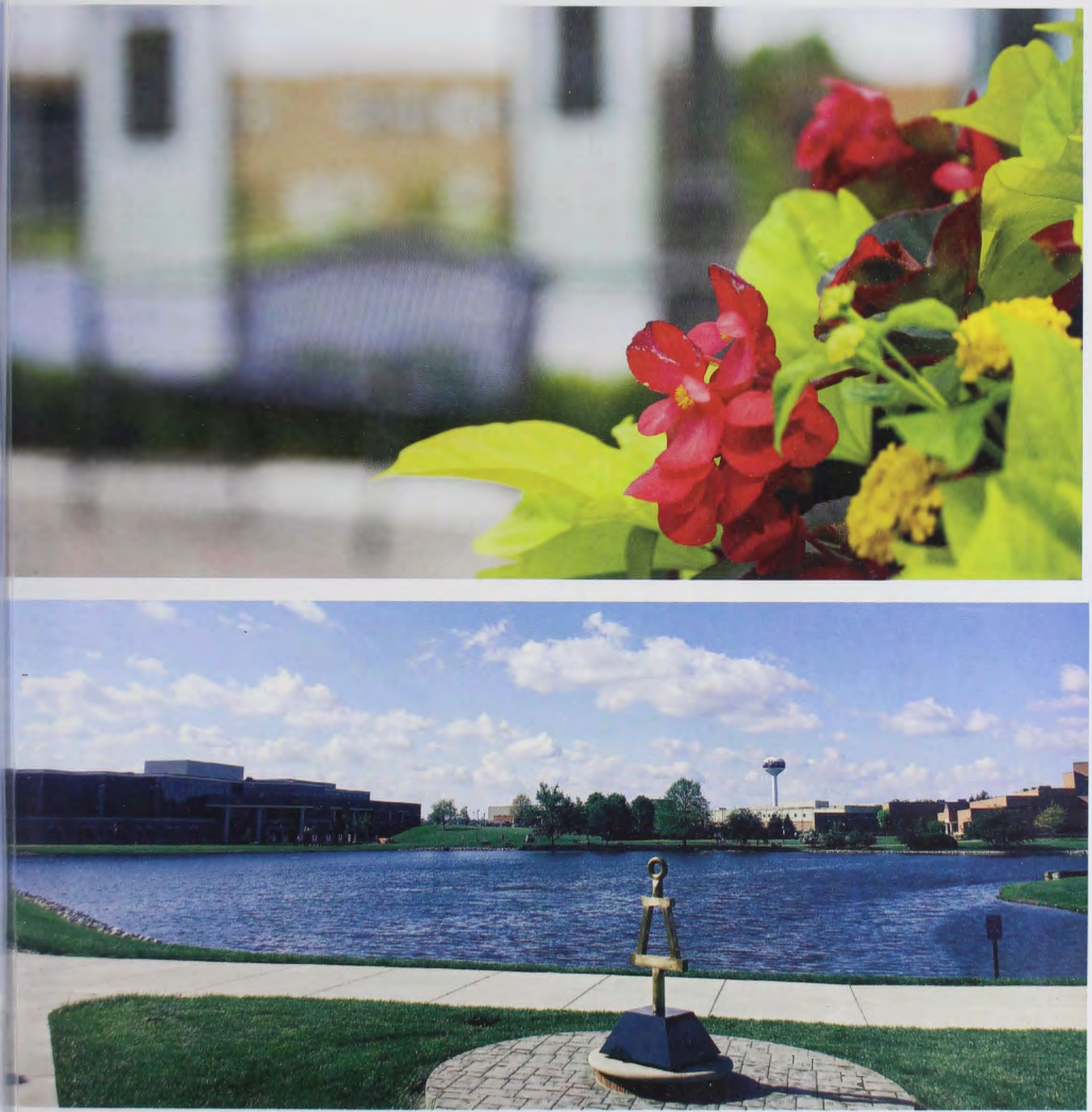

"And make us happy in the darting bird That suddenly above the bees is heard, The meteor that thrusts in with needle bill, And off a blossom in mid air stands still."
"For this is love and nothing else is love, The which it is reserved for God above To sanctify to what far ends He will, But which it only needs that we fulfill." 


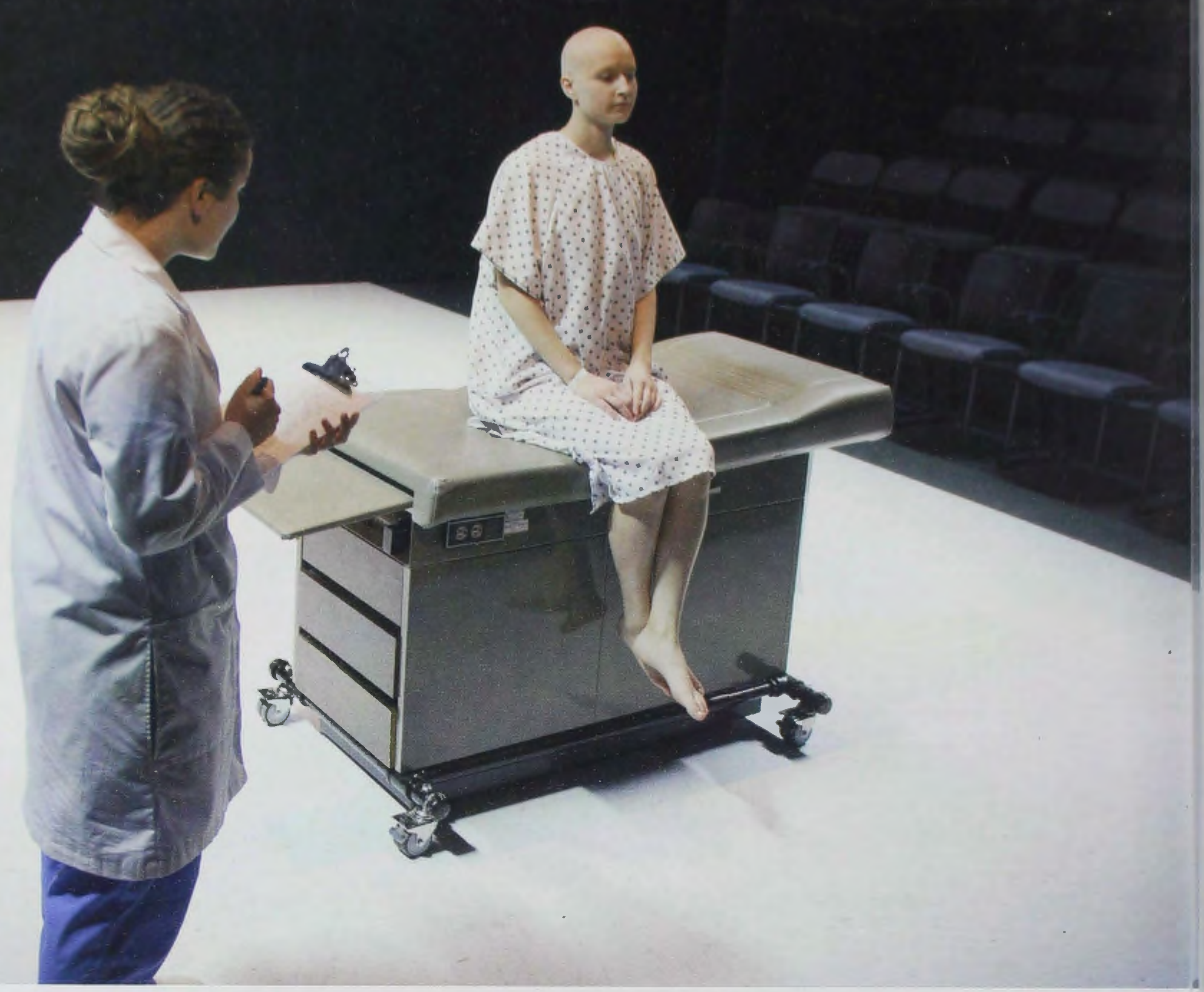

Wit

The last play of the school year was performed by the theatre department from March 31-April 10. The theatre department performed their second play of the spring on Wit. The story of Wit follows the life of a brilliant university professor who is diagnosed with terminal cancer. The play follows her life, through treatments which bring understanding of her work, as well as her life. Wit was such a captivating play because it touches on important aspects of life that affect everyone: pain, suffering, and death. It challenged the audience as well as the actors on the stage as to what things in life are so valuable that they are worth holding on to. 


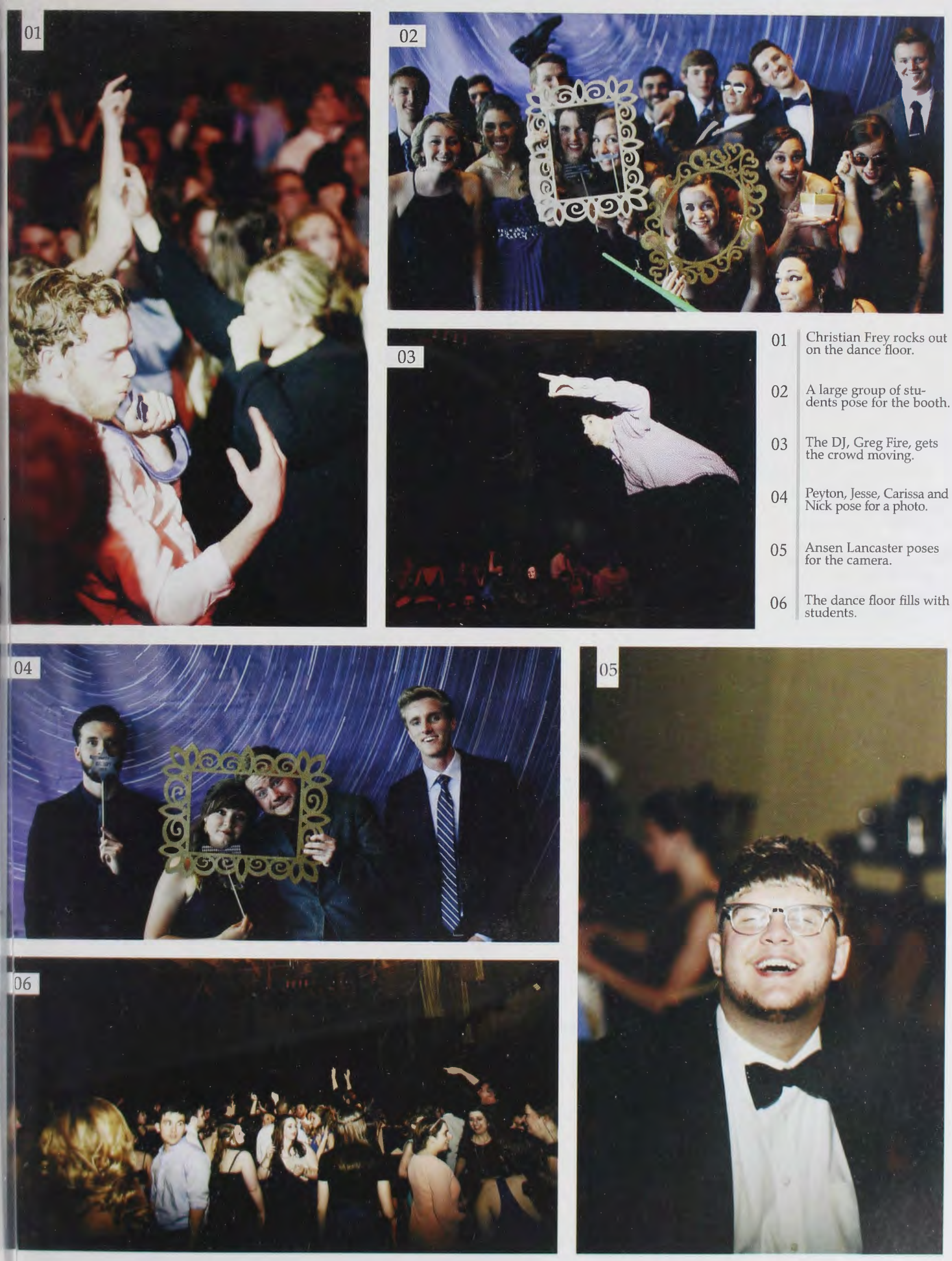



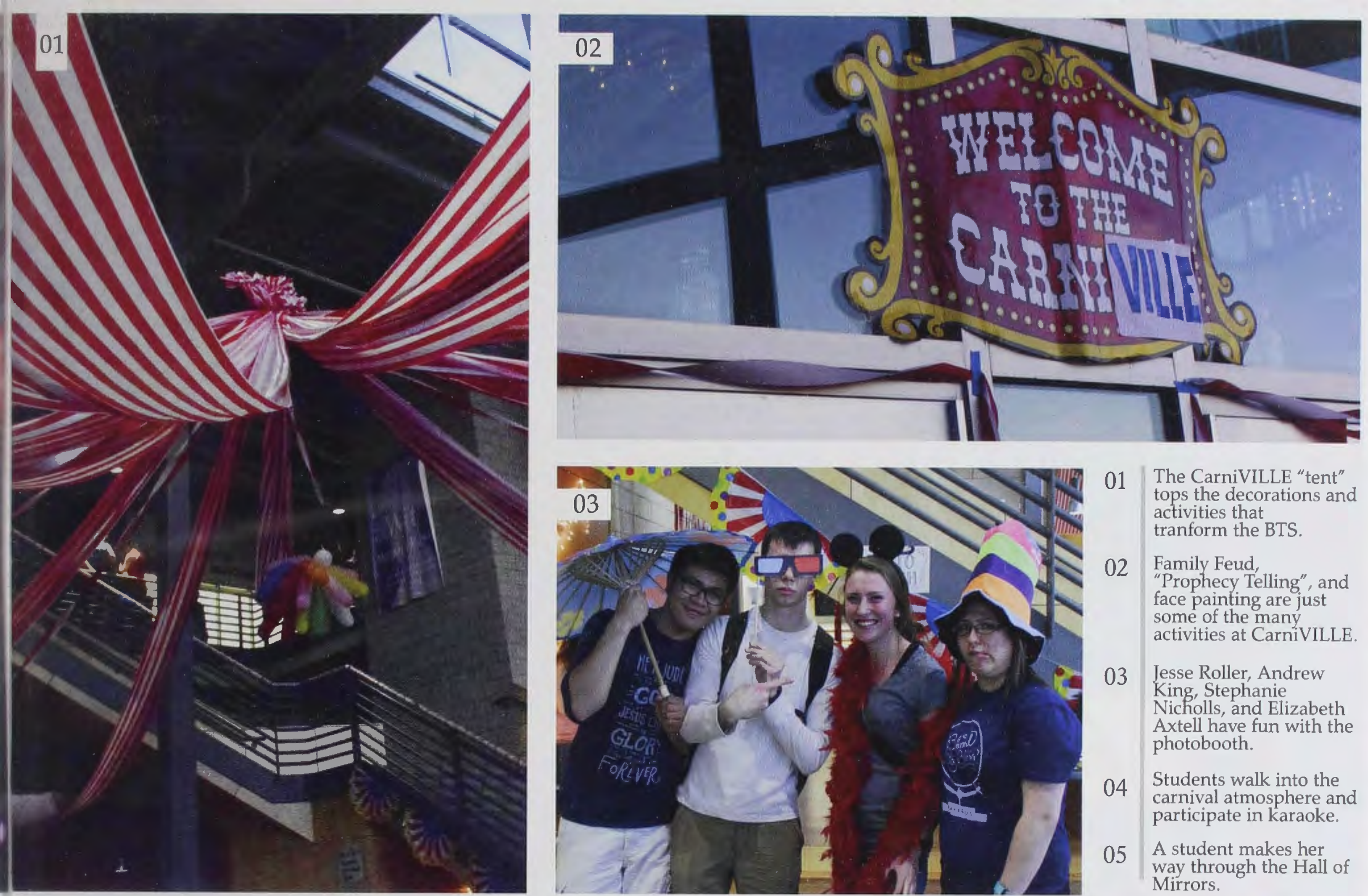

01 The CarniVILLE "tent" tops the decorations and activities that tranform the BTS.

02 Family Feud,

"Prophecy Telling", and face painting are just some of the many activities at CarniVILLE.

03 Jesse Roller, Andrew King, Stephanie

Nicholls, and Elizabeth Axtell have fun with the photobooth.

04 Students walk into the carnival atmosphere and participate in karaoke.

05 A student makes her way through the Hall of Mirrors.
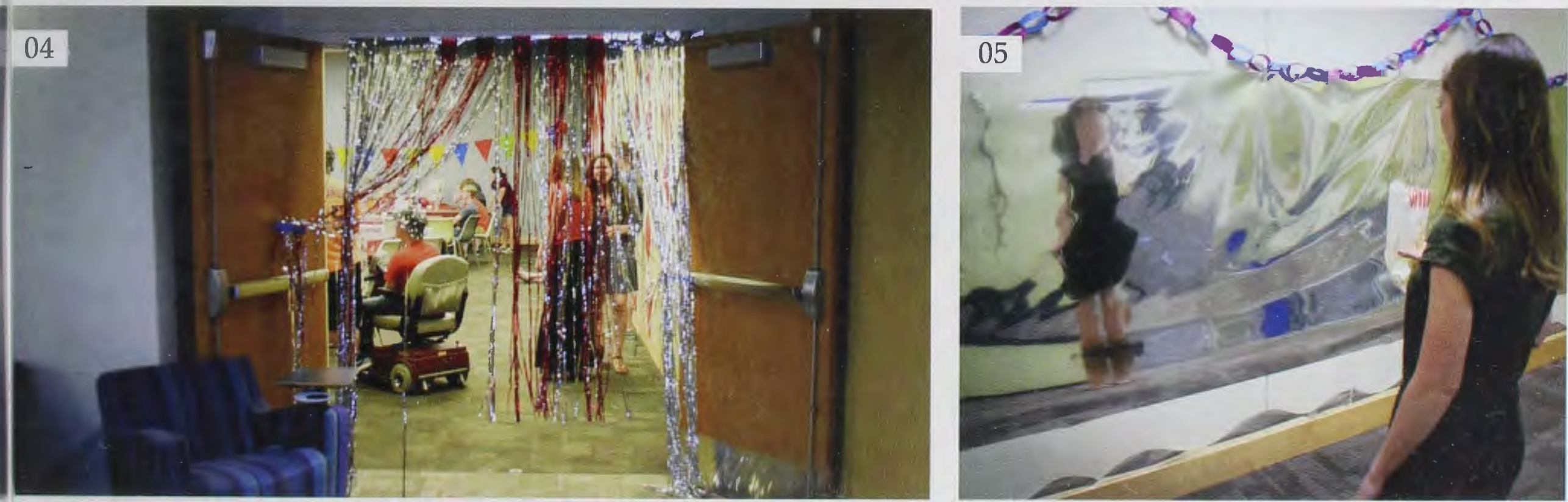

\section{SoFresh}

SoFresh is an event made just for sophomore and freshman students during JuniorSenior weekend. As the other students travelled to a dance, these students participated in activities held on campus. This year's theme was CarniVILLE, which was set up all over the Biblical and Theological Studies building. Members of both class councils came together to provide these students with a great weekend.

There were carnival games set up, food, and other activities for students to participate in. This was a totally free event for students not able to attend JuniorSenior. 

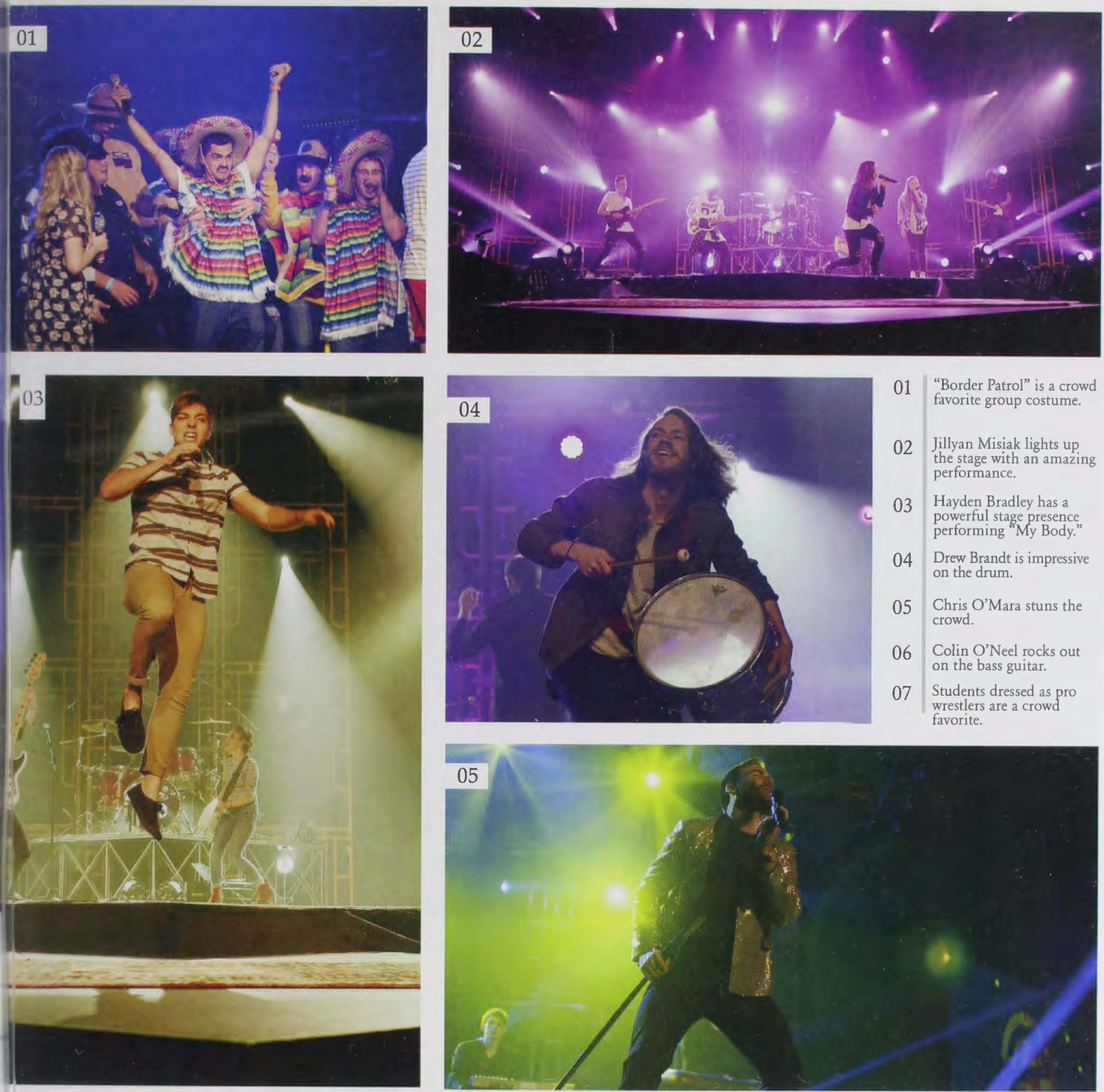

01 "Border Patrol" is a crowd favorite group costume.

02 Jillyan Misiak lights up the stage with an amazin performance.

03 Hayden Bradley has a powerful stage presence
performing "My Body."

04 Drew Brandt is impressive on the drum.

05 Chris O'Mara stuns the crowd.

06 Colin O'Neel rocks out on the bass guitar.

07 Students dressed as pro wrestlers are a crowd favorite.
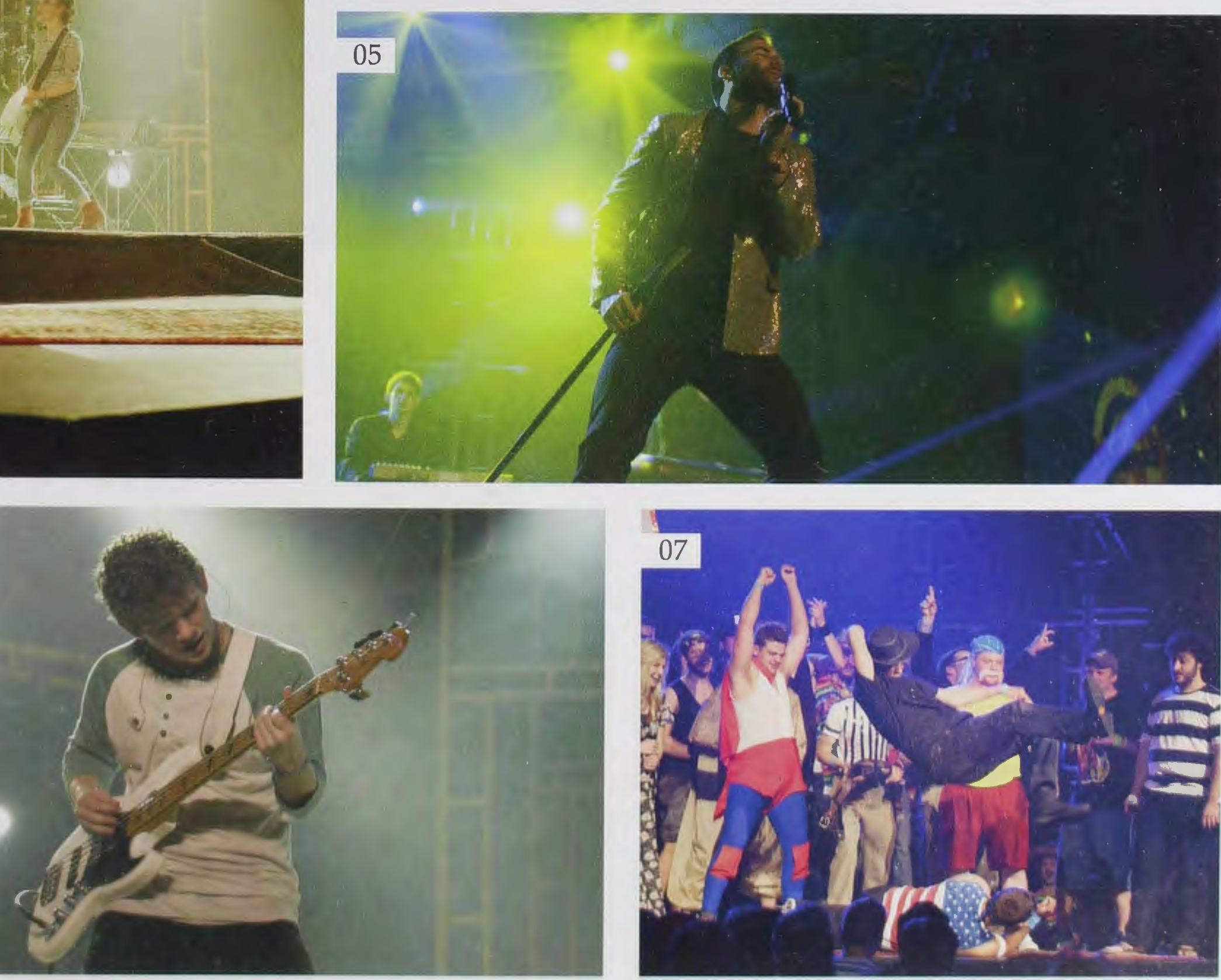


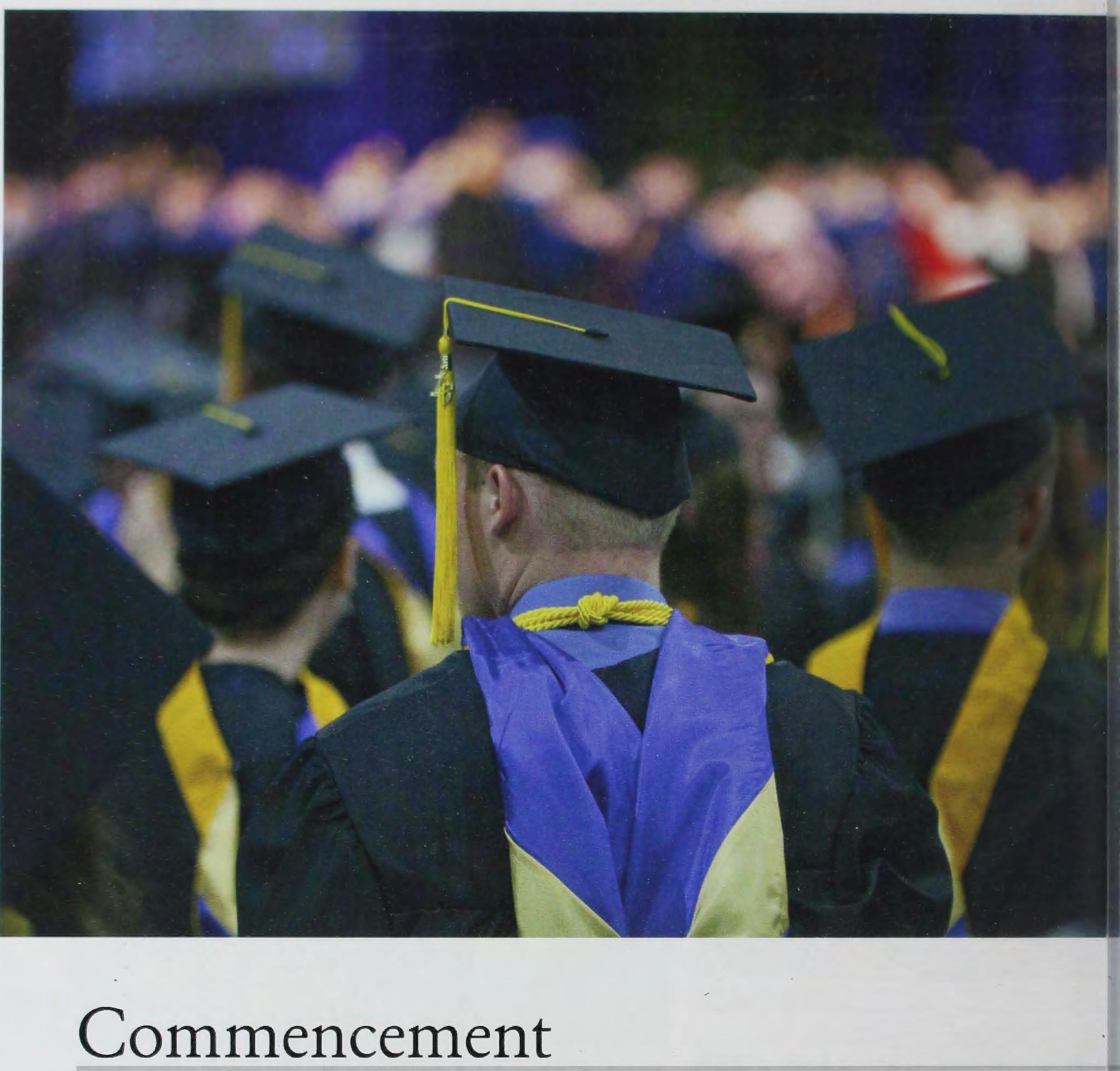

If you asked a senior during the spring semester how many days there were until graduation, you'll always get the answer, sometimes down to the minute. This year's commencement did not disappoint those who were excited. It was a fitting tribute to the individuals in the class and the work that they had done. This year's speaker was Dr. Thomas White. His challenging charge to the graduates encouraged them to be good at what they do, For the Glory of God.
It was perhaps the hymn that was sung that was the most fitting tribute to this class. "Be Still My Soul" was the hymn, and its words reminded the graduates of God's faithfulness in thier lives over the past four years. 


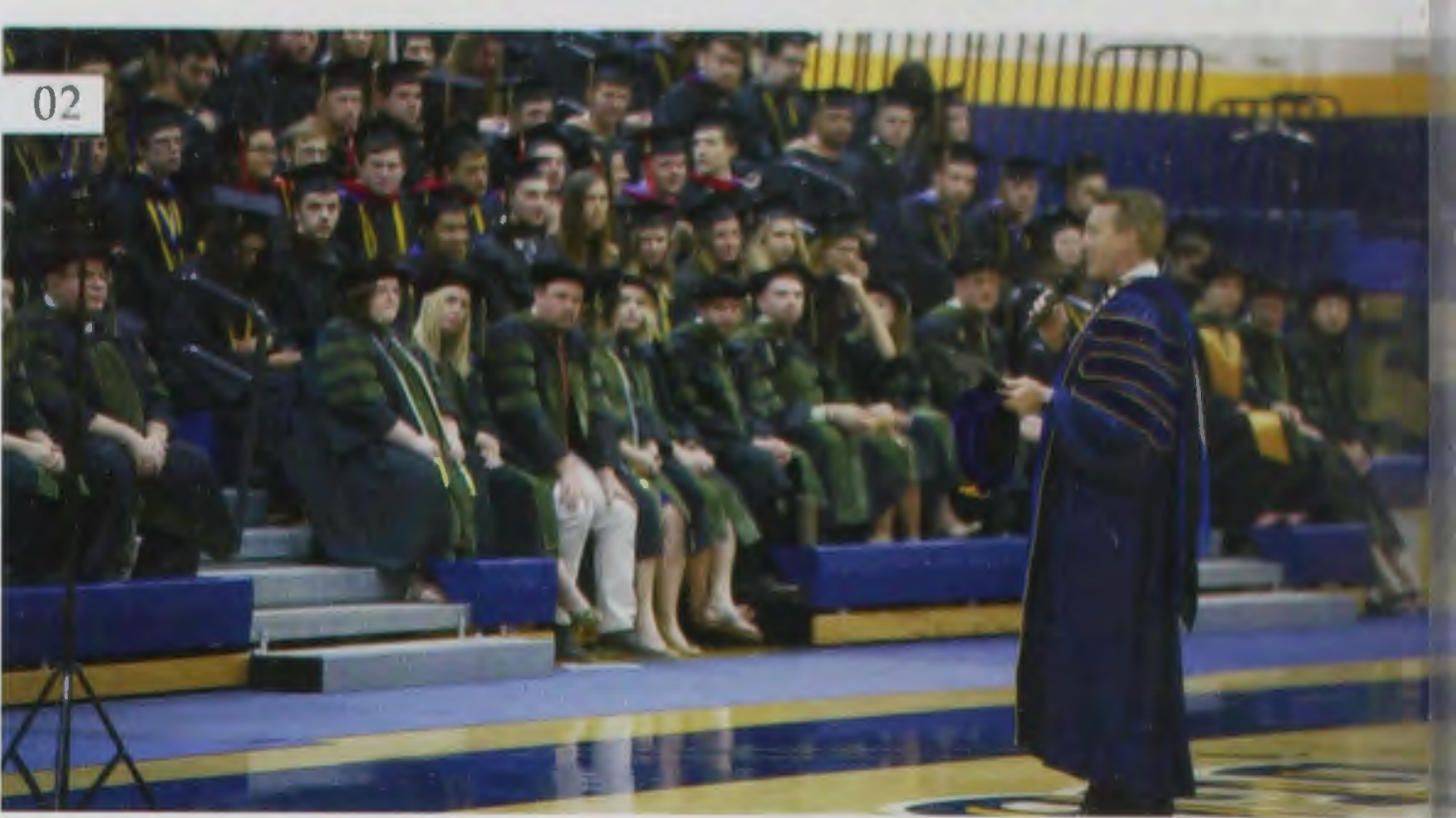
01 Class advisor Loren
Kuhn addresses the class
tor the last time.

Dr. White prepares students for the ceremony.

03 Proud parents take photos of their kids.

04 Every parent, grandparent, and friend wants a ticket to graduation.

05 SGA chaplain, Steve Clark, reads a passage of Scripture

Loren Reno gives a challenege to the class.
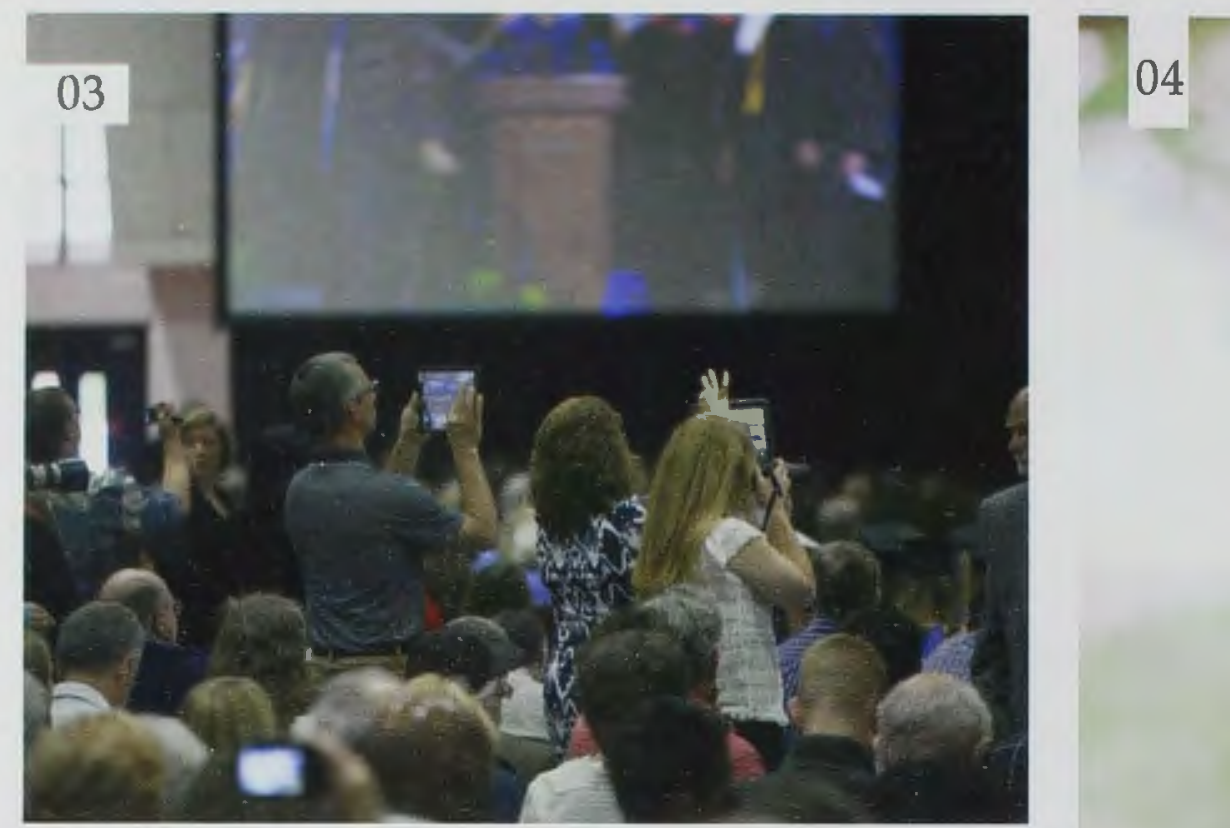

"Graduation is a chance to celebrate each little piece of Cedarville that four years ago we had no idea we couldn't have lived without. God turned strangers into family, and the hardest times into lessons, but mostly Ohio into home."

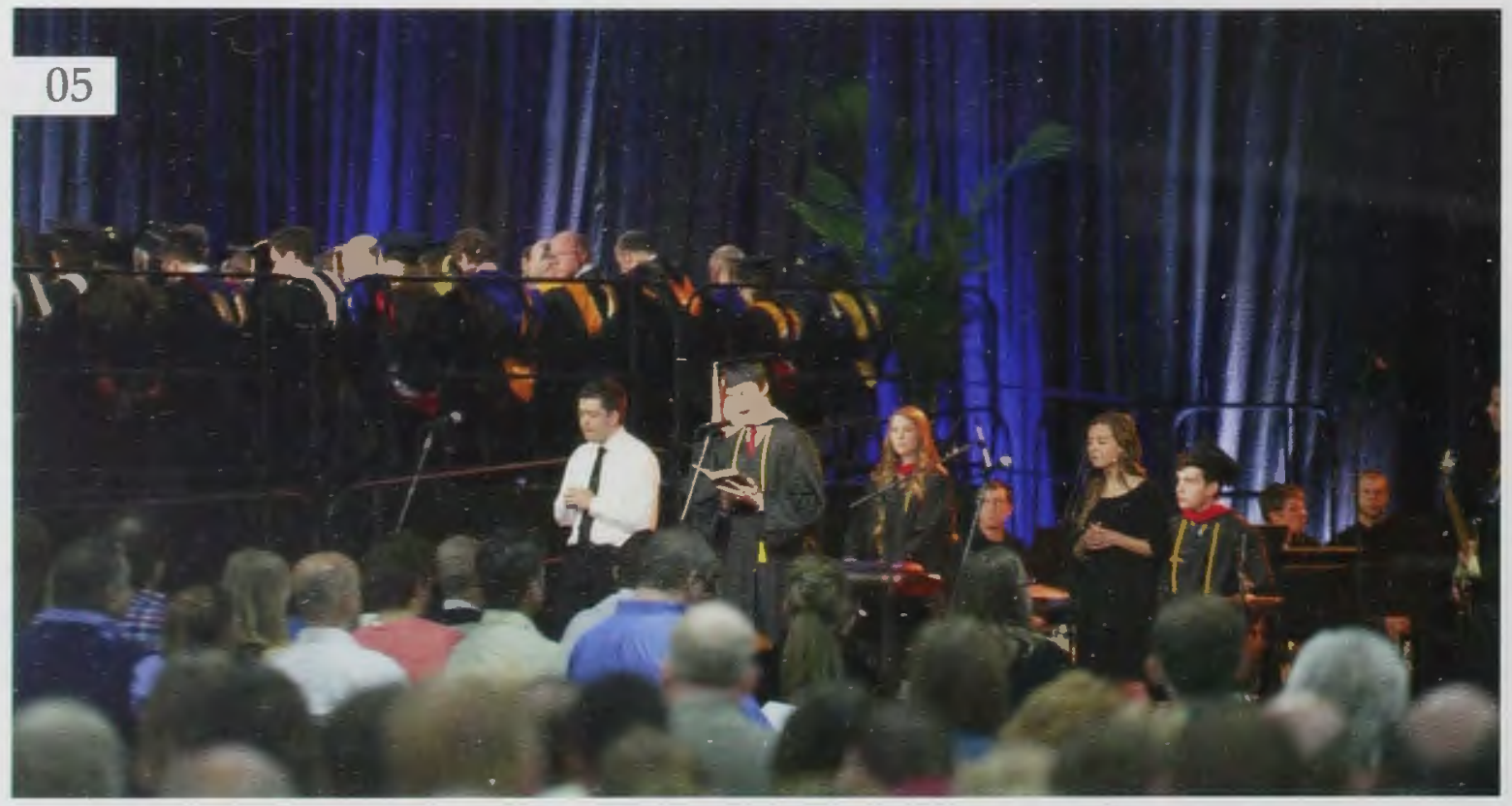

06

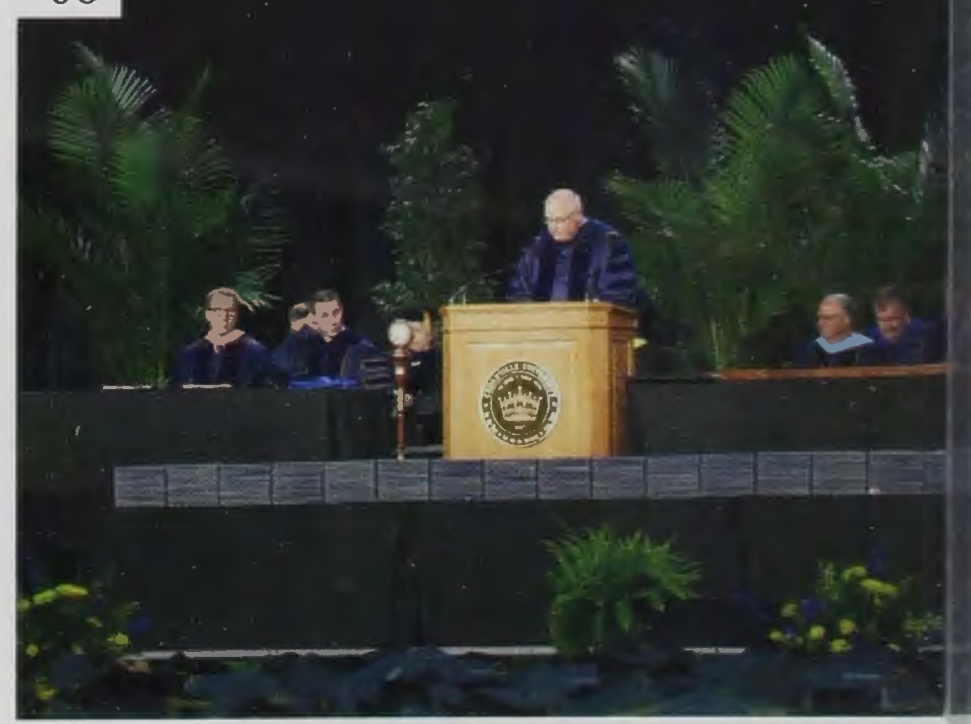




\section{student life}

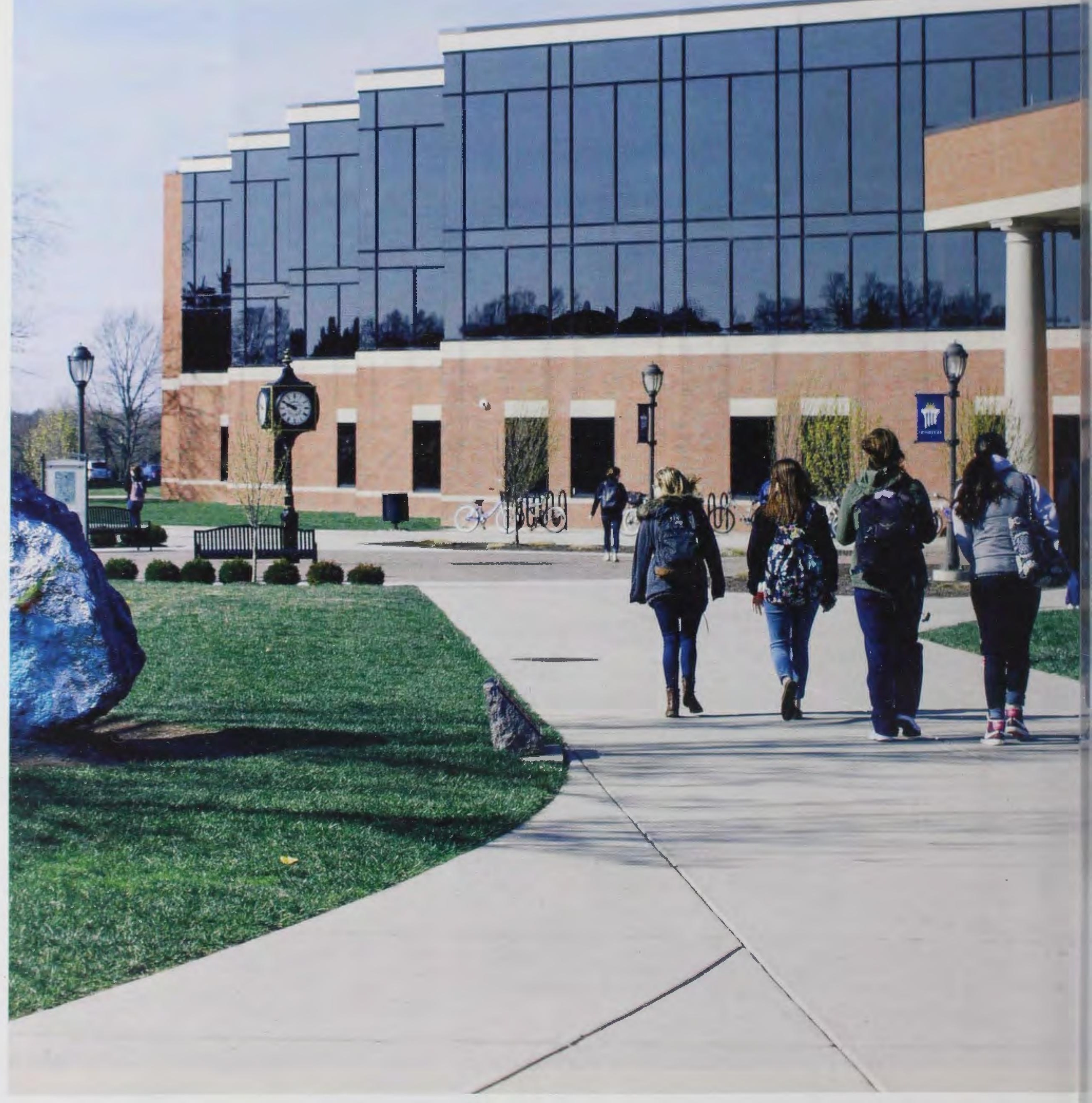




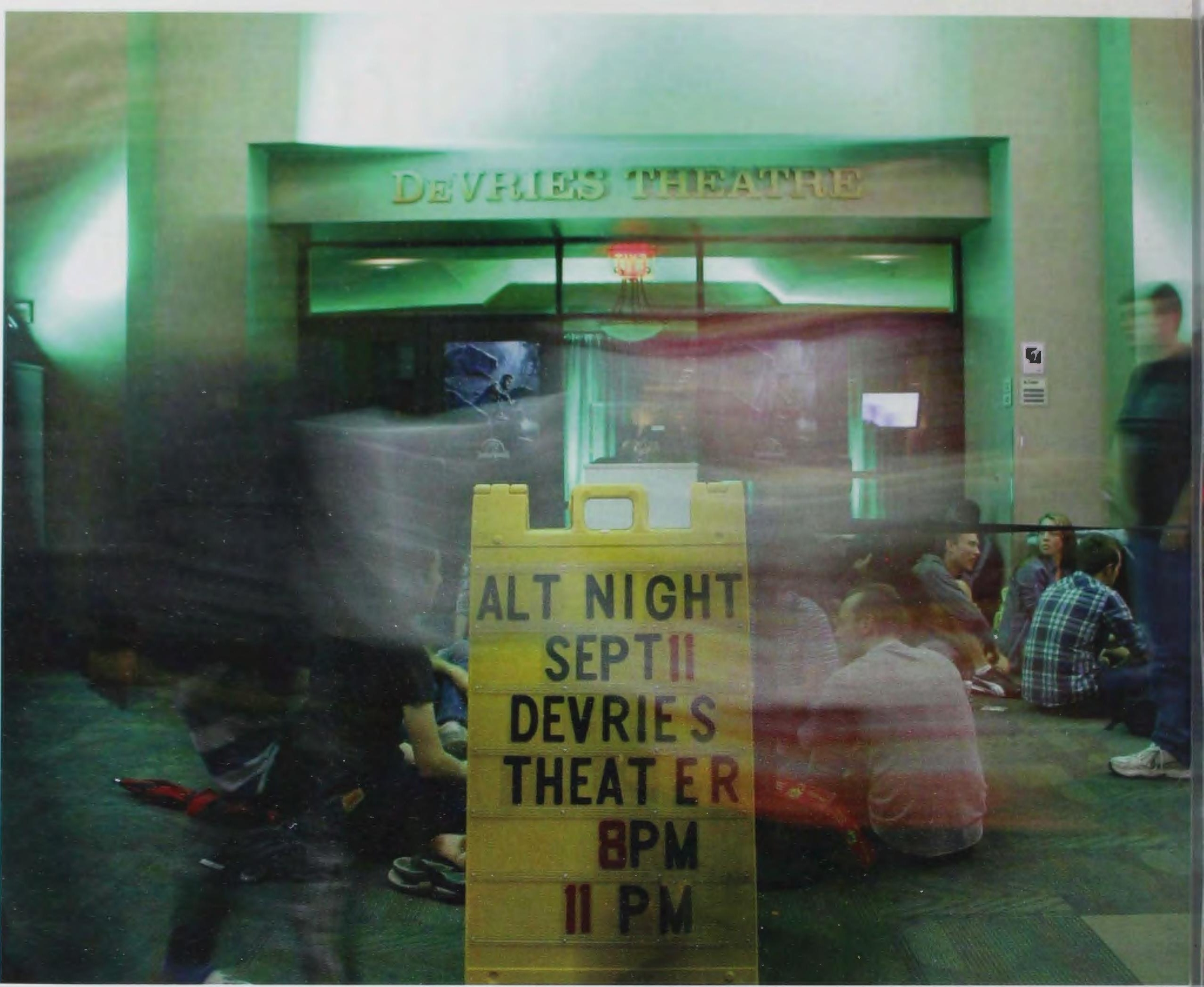

\section{ALT Night}

It is easy to get a little stir-crazy in these corn fields - and sometimes hard to find entertainment to alleviate that. For the weekends of freedom from papers, exams, and projects, the
Student Center

Activities Board provided an alternative to spending that last check from parents or just taking a three-hour nap. Alt. nights gave students an opportunity to get their heads out of their textbooks and spend time with their friends without breaking the bank, providing movies and food and fun activities without students having to leave campus. 

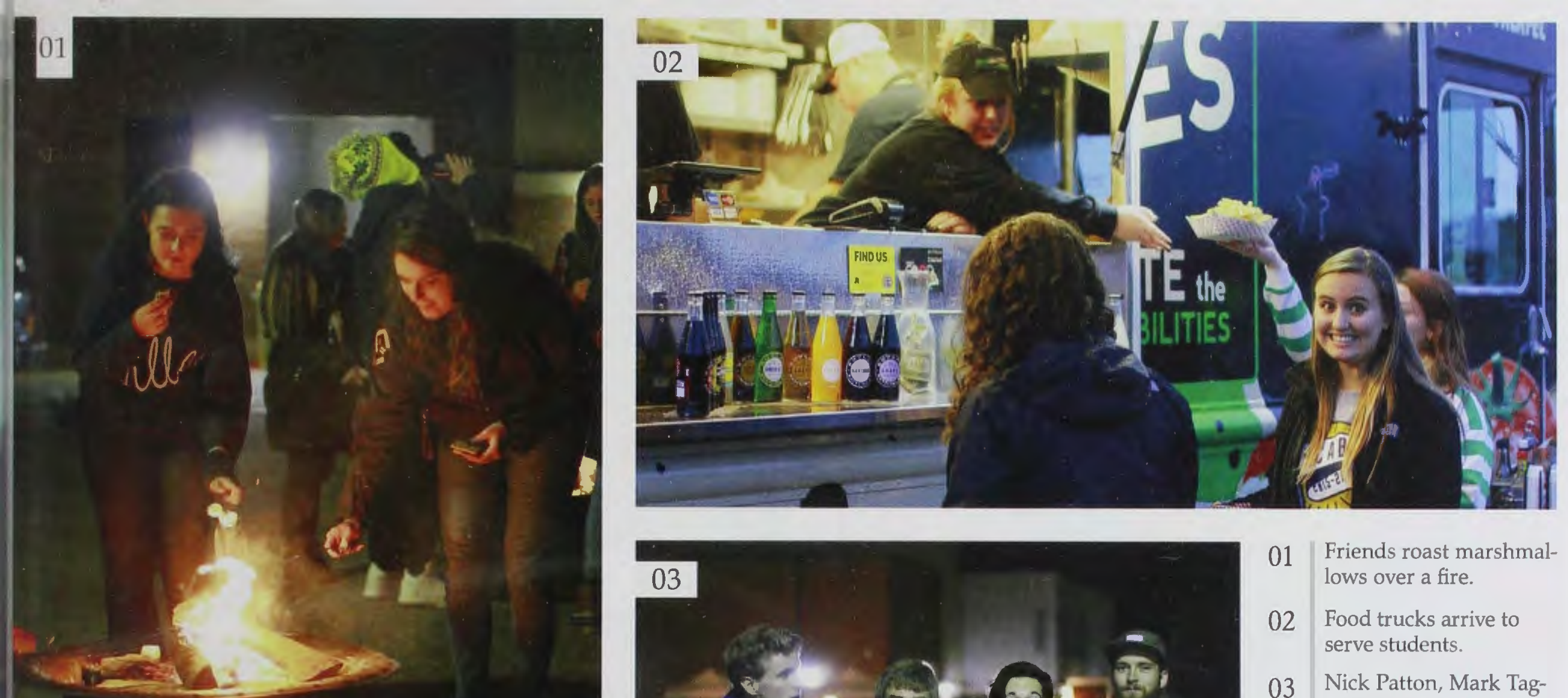

\section{3}

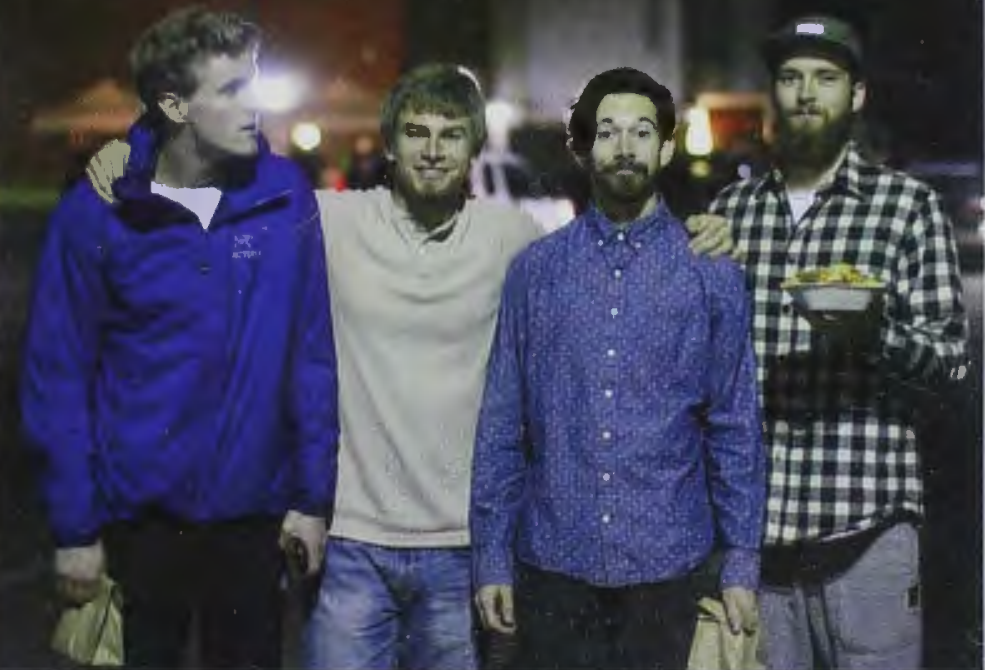

01

02

03

04 Student Life staff have a boxing match

05 Students and little sibs become archers for Catching Fire.

06 Students play Dutch Blitz while waiting in line.

07 Friends enjoy the Autumn air and a Hive meal.
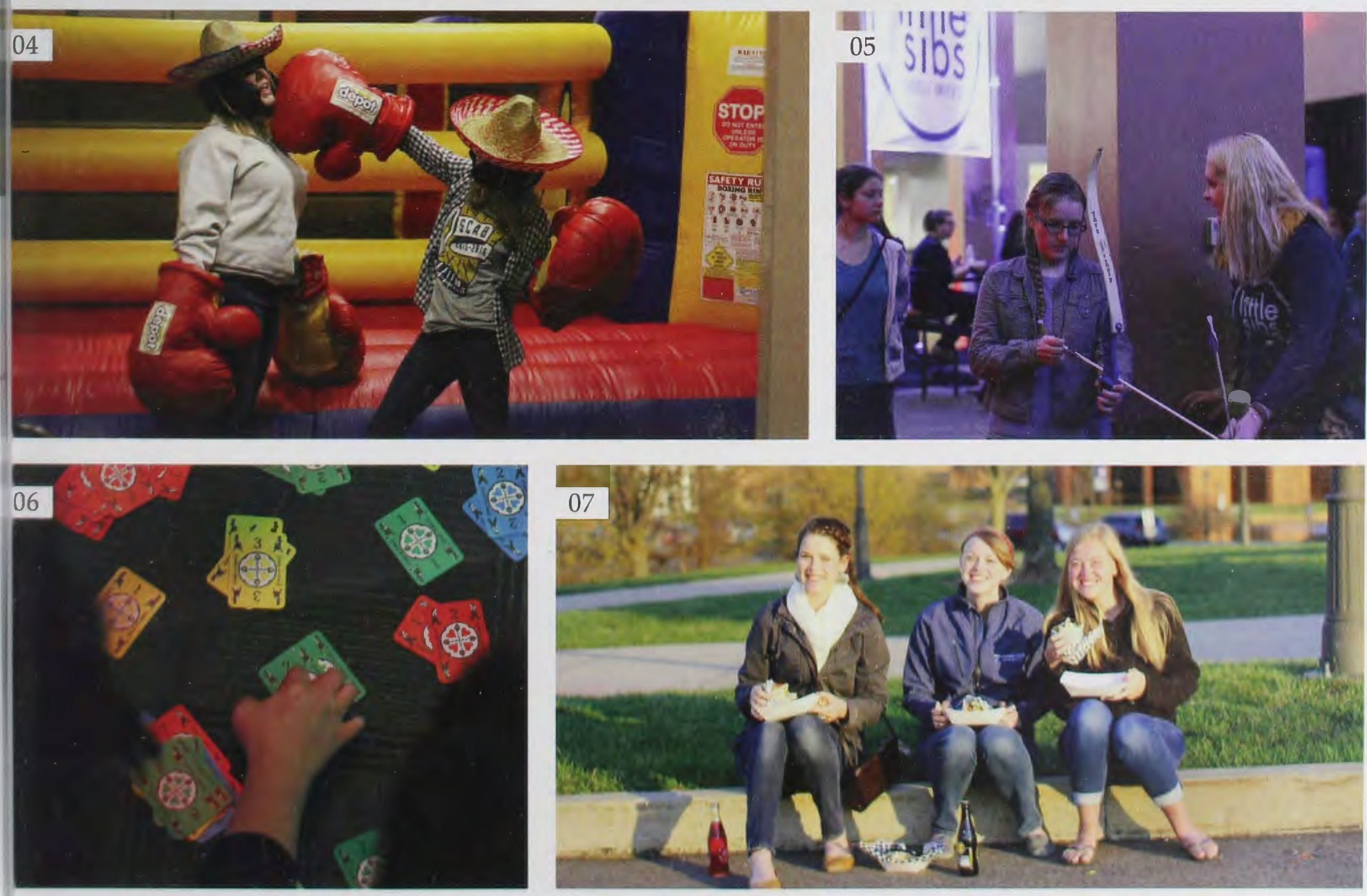


\begin{tabular}{l|l}
01 & $\begin{array}{l}\text { One student faces the } \\
\text { dreaded Fear Factor } \\
\text { challenge. }\end{array}$ \\
02 & $\begin{array}{l}\text { DTK demands to be } \\
\text { taken seriously. }\end{array}$ \\
03 & $\begin{array}{l}\text { An interpretive dance } \\
\text { intimidates foes. }\end{array}$ \\
04 & $\begin{array}{l}\text { Some housewives } \\
\text { prepare for the wars. }\end{array}$ \\
05 & $\begin{array}{l}\text { Members of OPE } \\
\text { watch the action. }\end{array}$ \\
06 & $\begin{array}{l}\text { Org costumes show } \\
\text { their spirit. }\end{array}$ \\
07 & $\begin{array}{l}\text { Orgs prepare for a } \\
\text { messy challenge. }\end{array}$
\end{tabular}

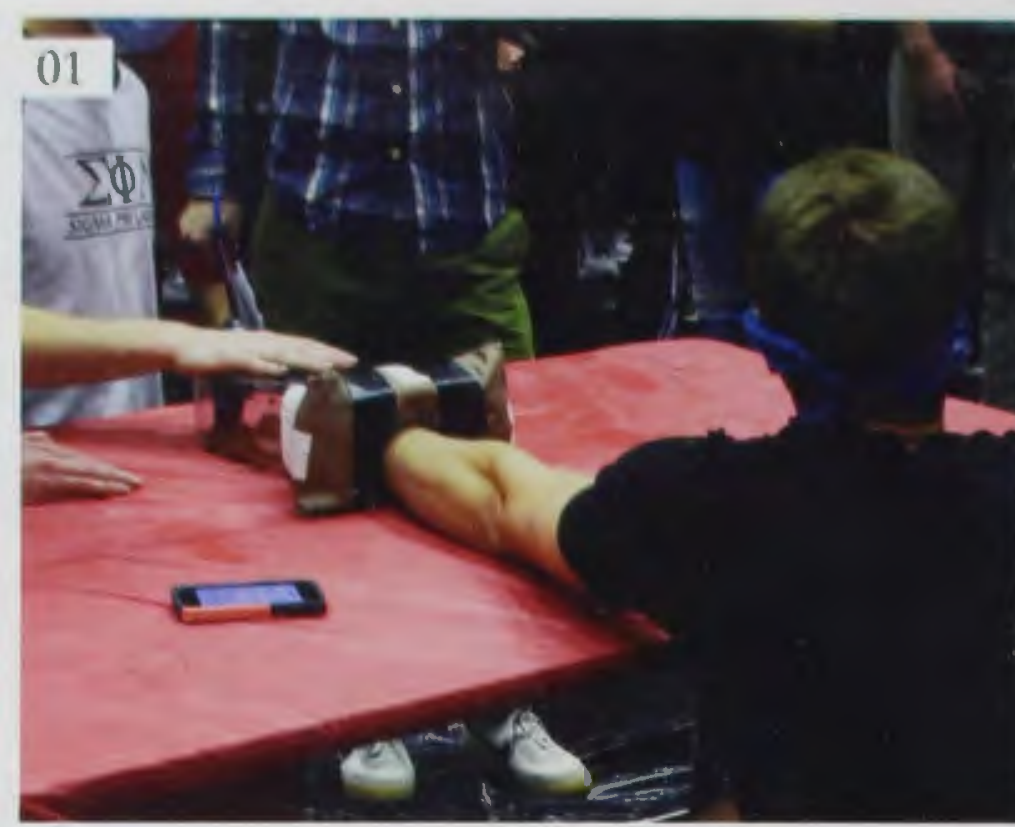

03

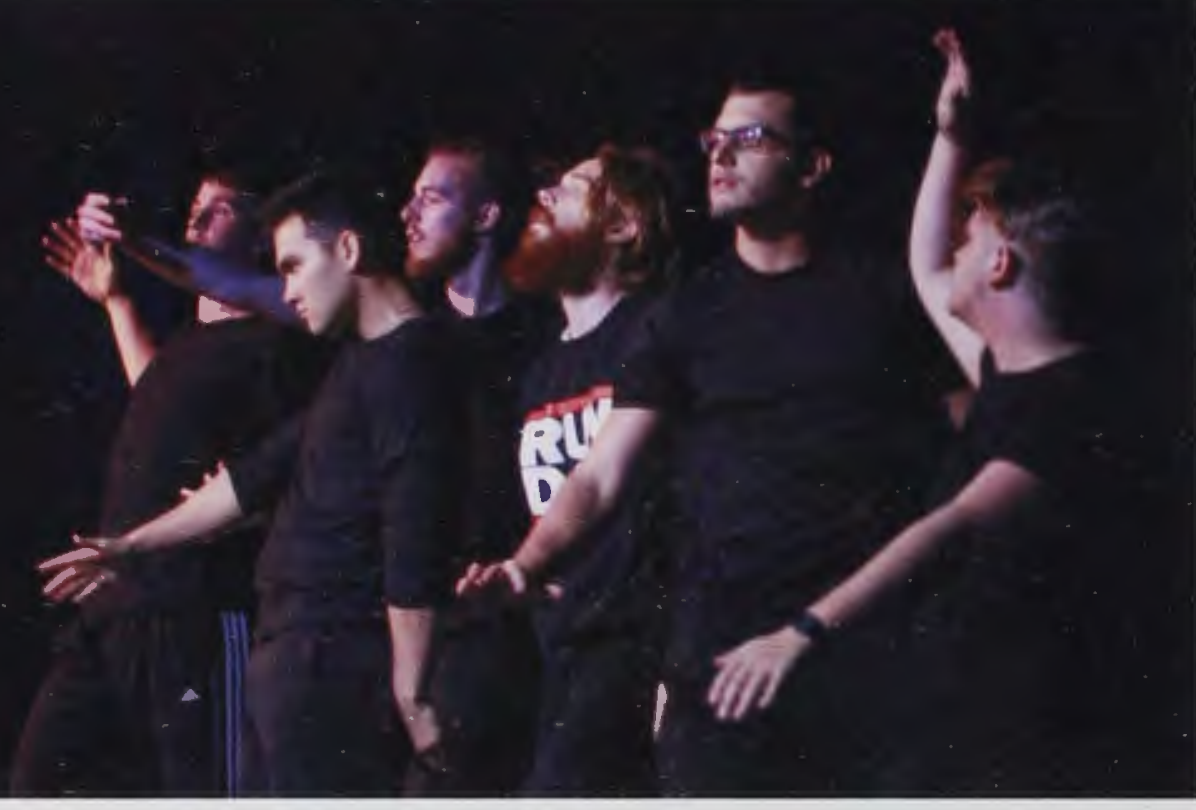

"Even though the road was a bit rocky, I think it was an engaging experience that gave our org an opportunity to grow closer and really come together for some really fun challenges. It is most definitely one of my most memorable experiences at Cedarville."
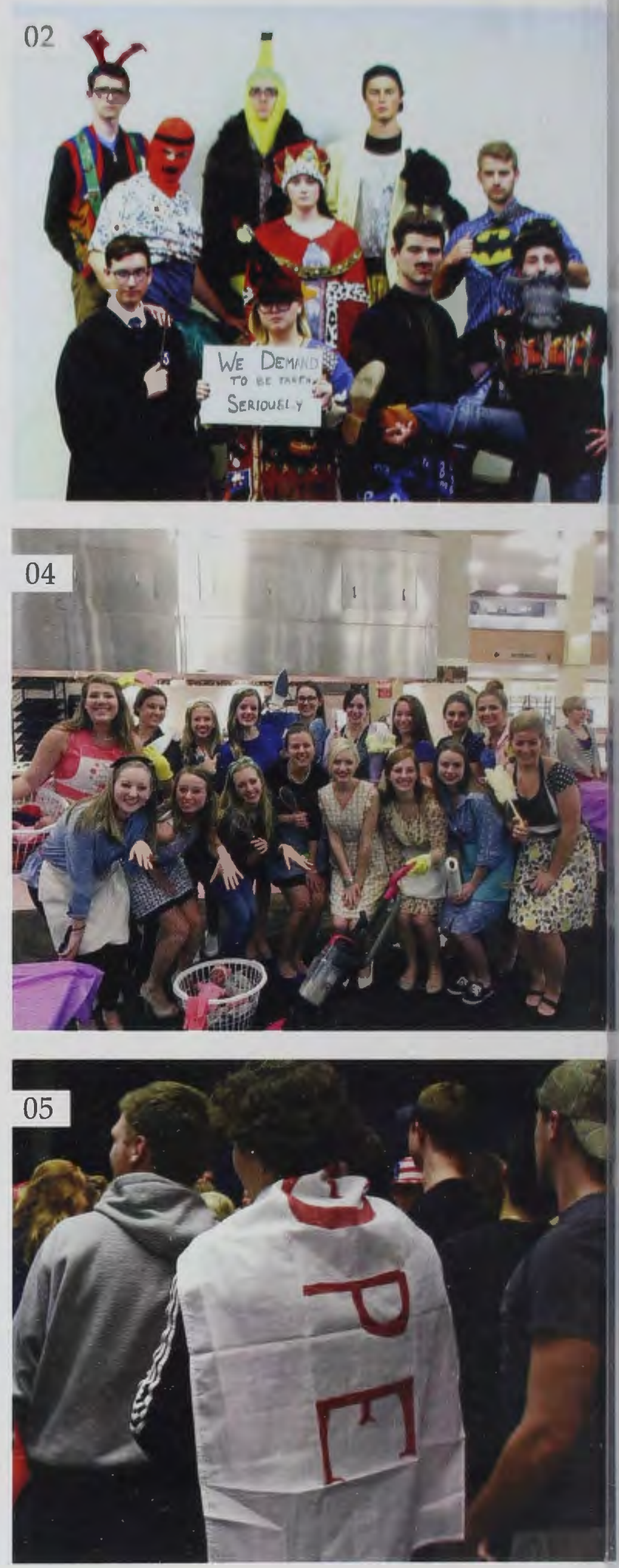
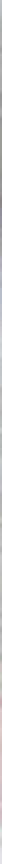

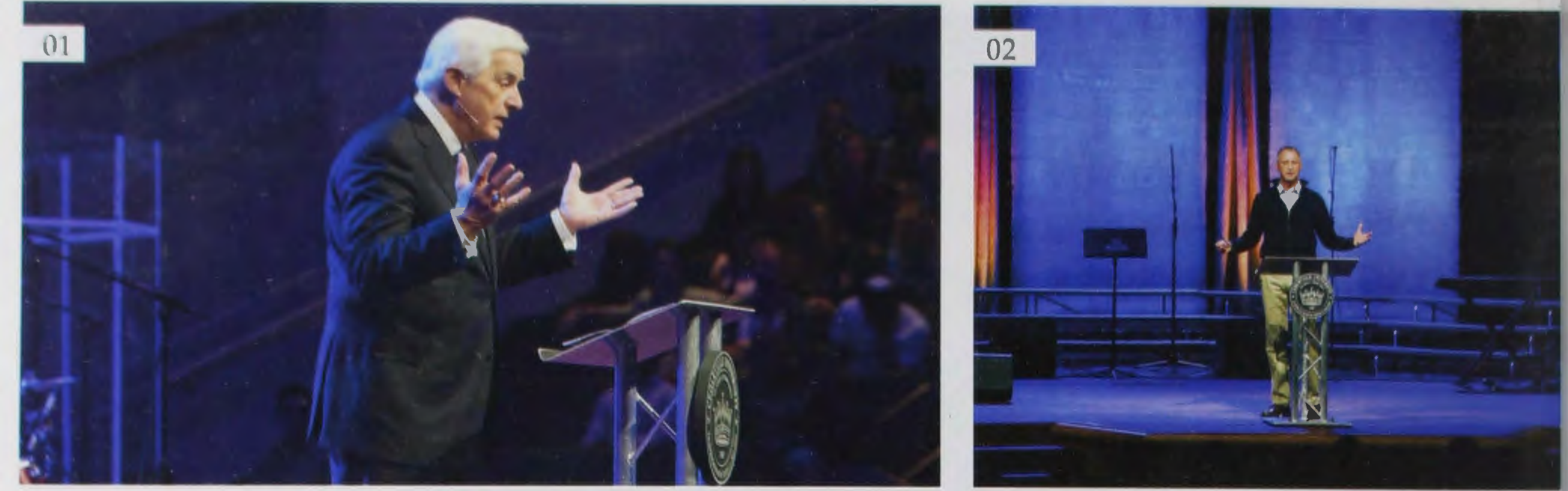

01 Dr. David Jeremiah preaches from Isaiah 40:31.

02 Duke Crawford, Senior Pastor of Emmanuel Baptist Church.

03 Rev. James D. Parker speaks on "What the Love of God Does."

04 Dr. Rob Turner, Lead Teaching Pastor at Apex Community Church.

05 Dr. Jon Wood, VP for Student Life and Christian Ministries.
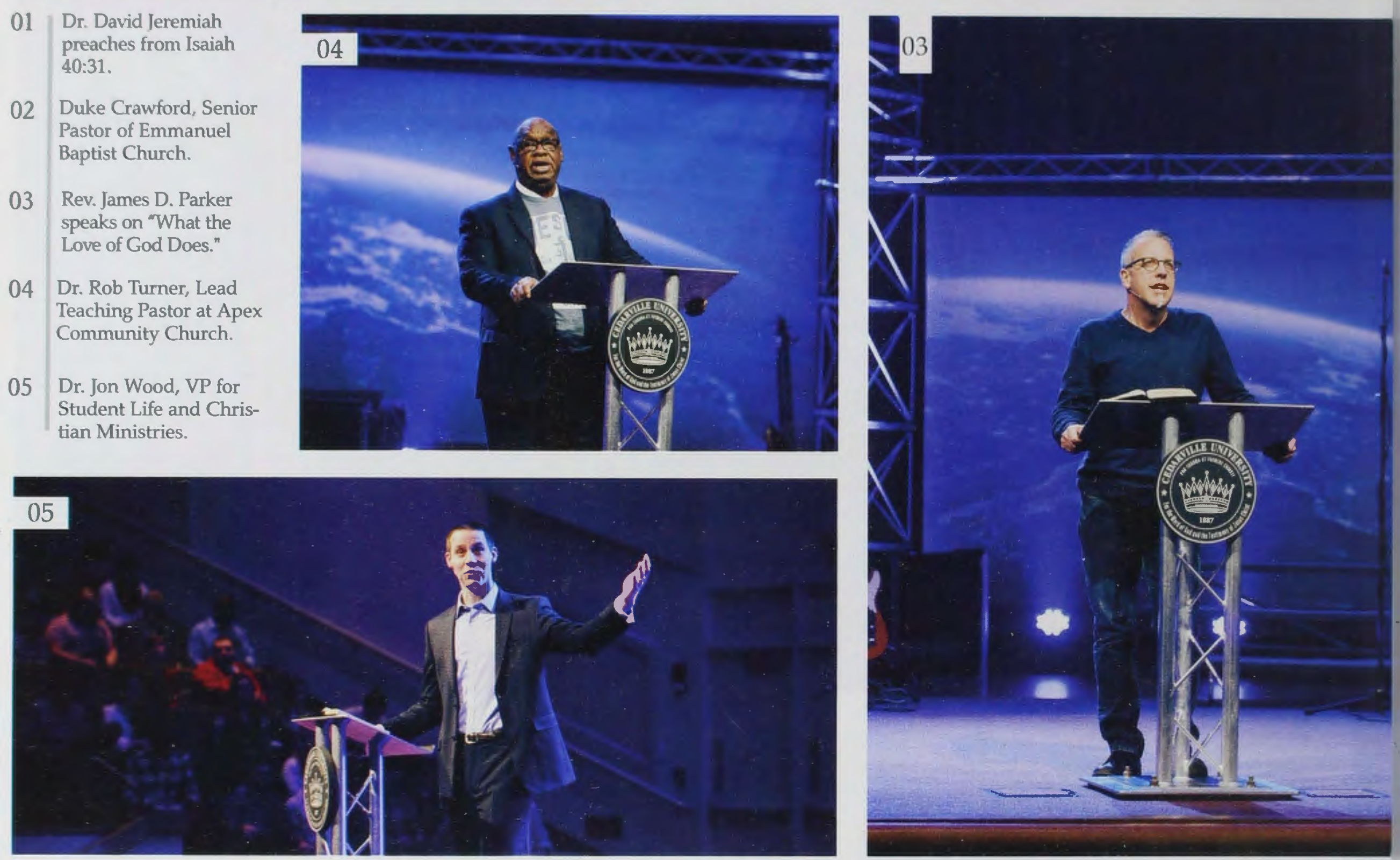

\section{Chapel}

For many students, chapel holds a significant place in the memories of their time at Cedarville. Each weekday at $10 \mathrm{am}$, students, faculty, and staff headed over to the DMC for a time of worship, reflection, and teaching.
Because of Cedarville's commitment to enriching students both intellectually and spiritually, speakers from around the world came to share parts of their lives and pieces of their wisdom. Throughout the year, our very own Dr. White presented his thoughtprovoking series, "We

Believe." Overall, chapel was the constant, unwavering source of fellowship and knowledge that brought students closer to each other and to their Savior throughout their years at Cedarville. 


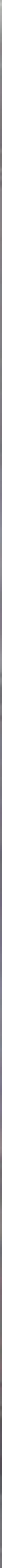

\section{Concerts}

One of Cedarville

University's core values

is Excellence in Effort.

That standard is no different when it comes to the performing arts, especially musically. Cedarville's music program allows students who study in all majors to be a part of music groups, as well as those who are studying their passion. The culmination of these efforts was captured throughout the year in their concerts. One of the biggest concerts of the year was the annual POPS concert. The POPS Concert was a tribute to Great Britain and the influence they have had on music. The concert featured nearly all of the choirs and bands at the university. In an effort to pay full tribute to Great Britain, they even finished with the National Anthem of Britain. Concerts are just another way students at Cedarville can express themseves. 


\section{sports}

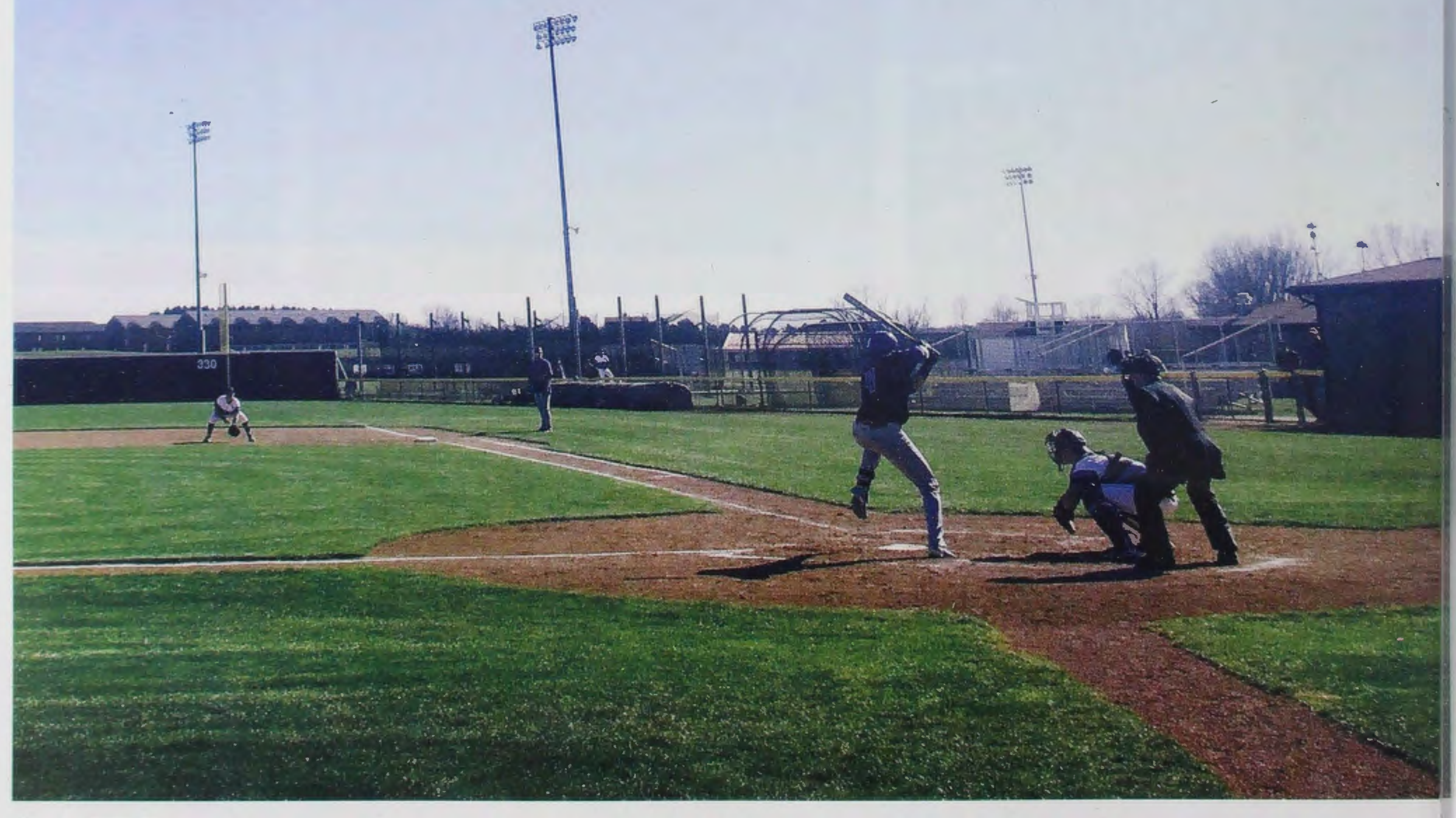




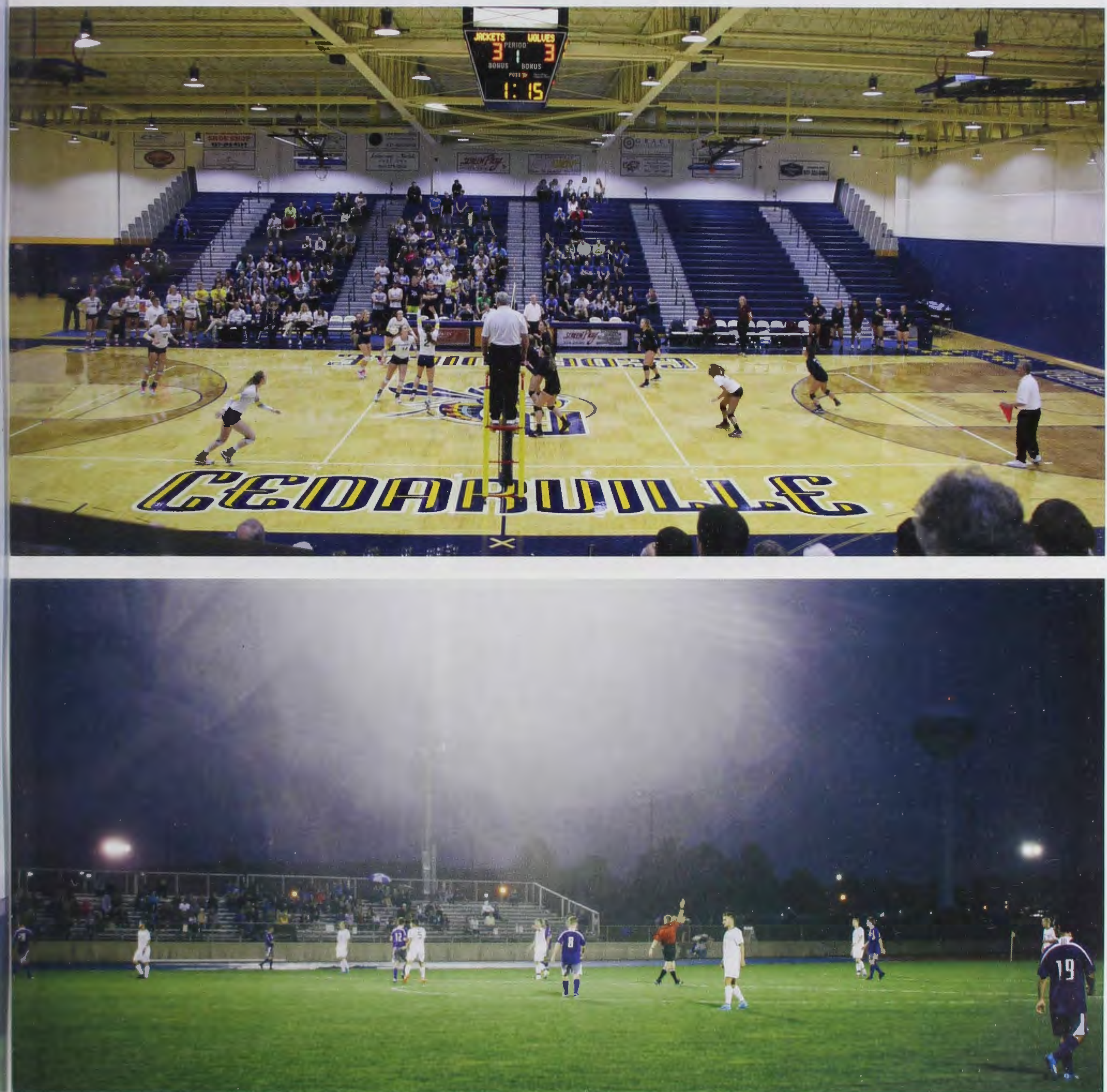

"We all have dreams. But in order to make dreams come into reality, it takes an awful lot of determination, dedication, self discipline, and effort." 

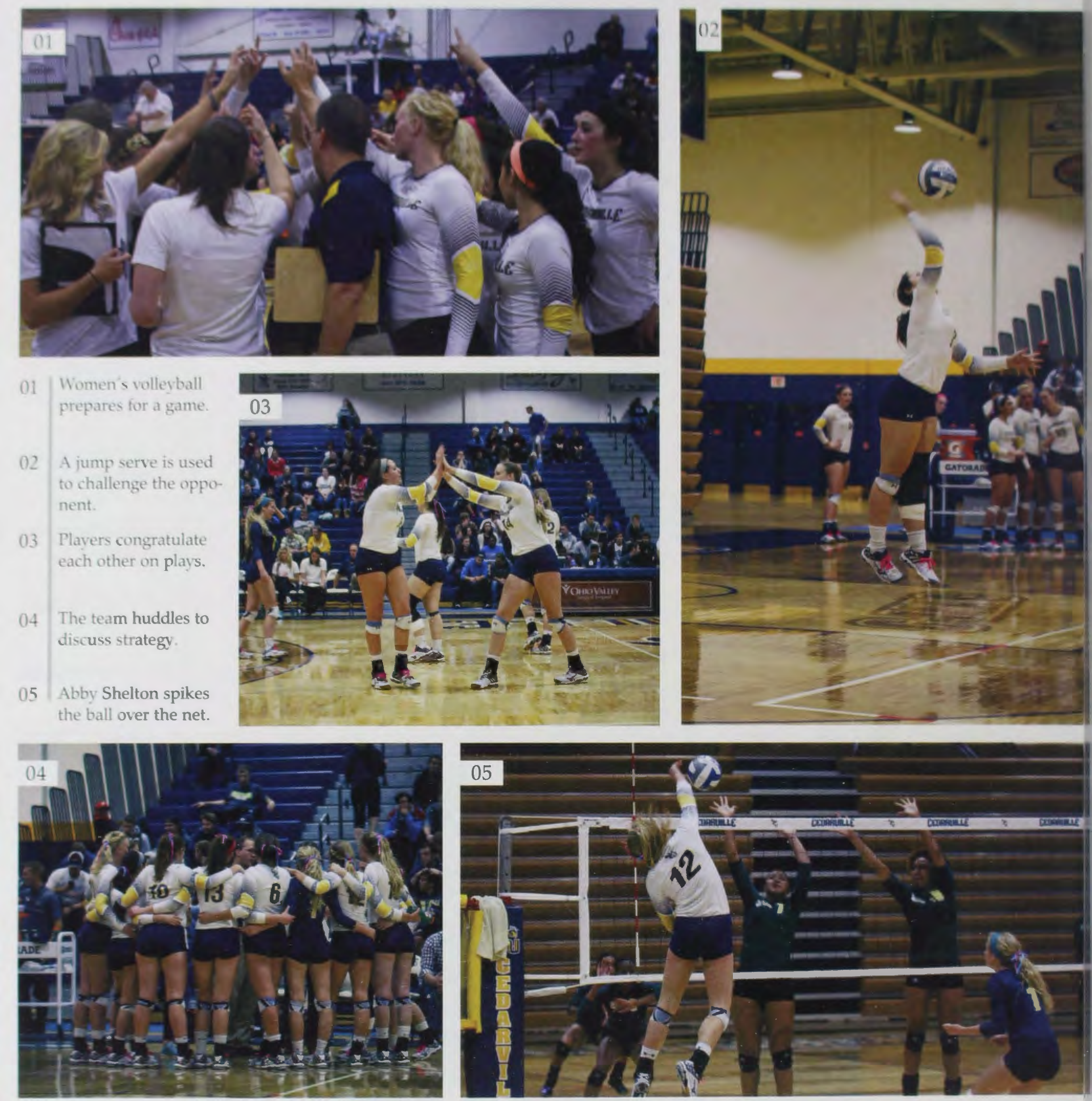

\section{Volleyball}

" The best part of the season was going 9-0 at home. It was really cool being able to form relationships with not only my teammates but also girls from other teams." 


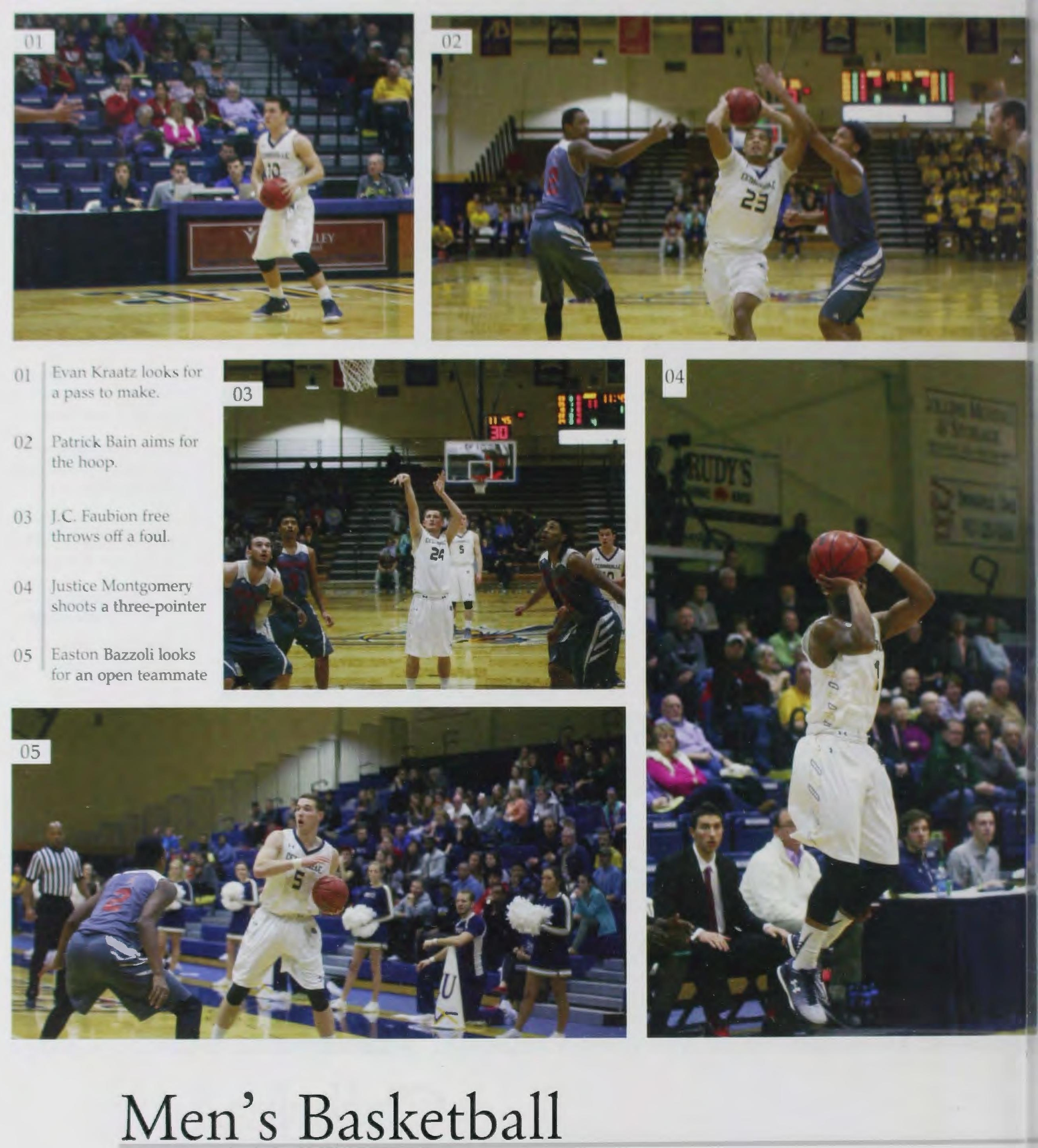

"I feel like one of the gifts God has given me is my athletic ability. I feel like I am glorifying Him when I play for Him." 


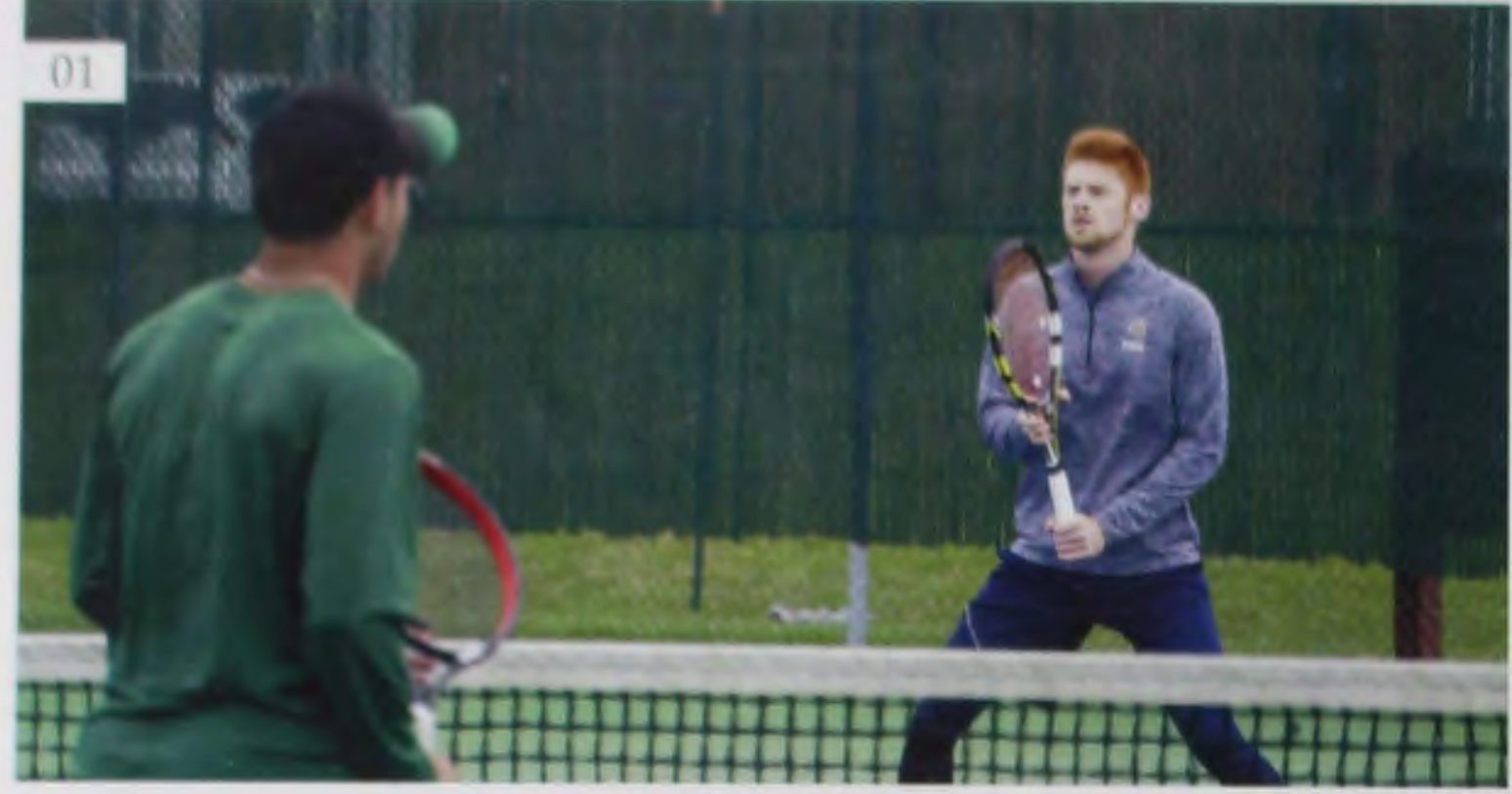

01 | Jonathan Class prepares for the serve.

02 Matt Brumbaugh returns the volley.

03 Stephen Combs concentrates during a home match.

04 Ryan Shomo extends his racket to hit the ball.

05 Joel Clark backhands a winner.
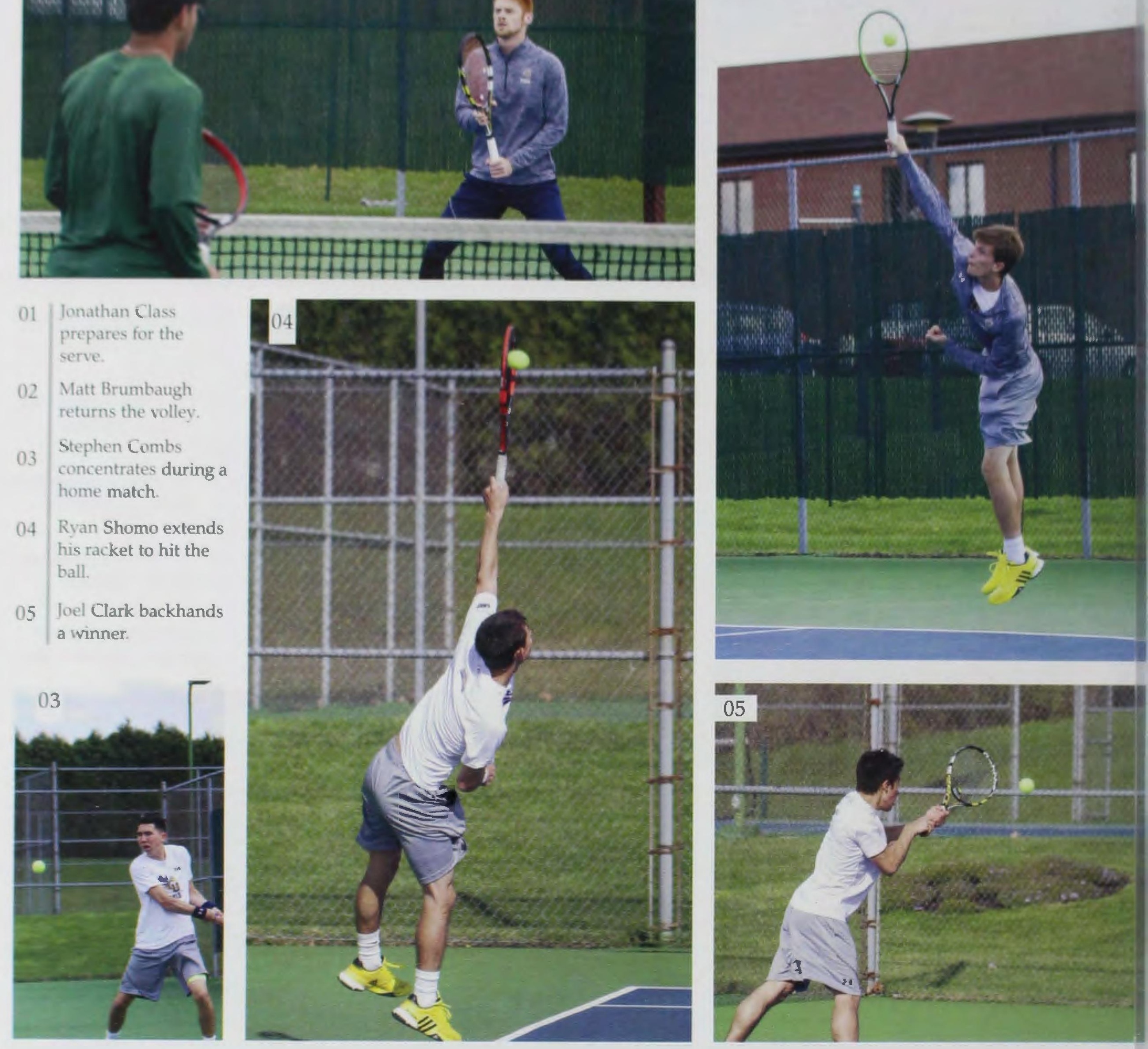

\section{Men's Tennis}

"We had a really good season. We were able to to win the G-MAC for the first time in Cedarville's history, and that just pushes us towards our next season." 

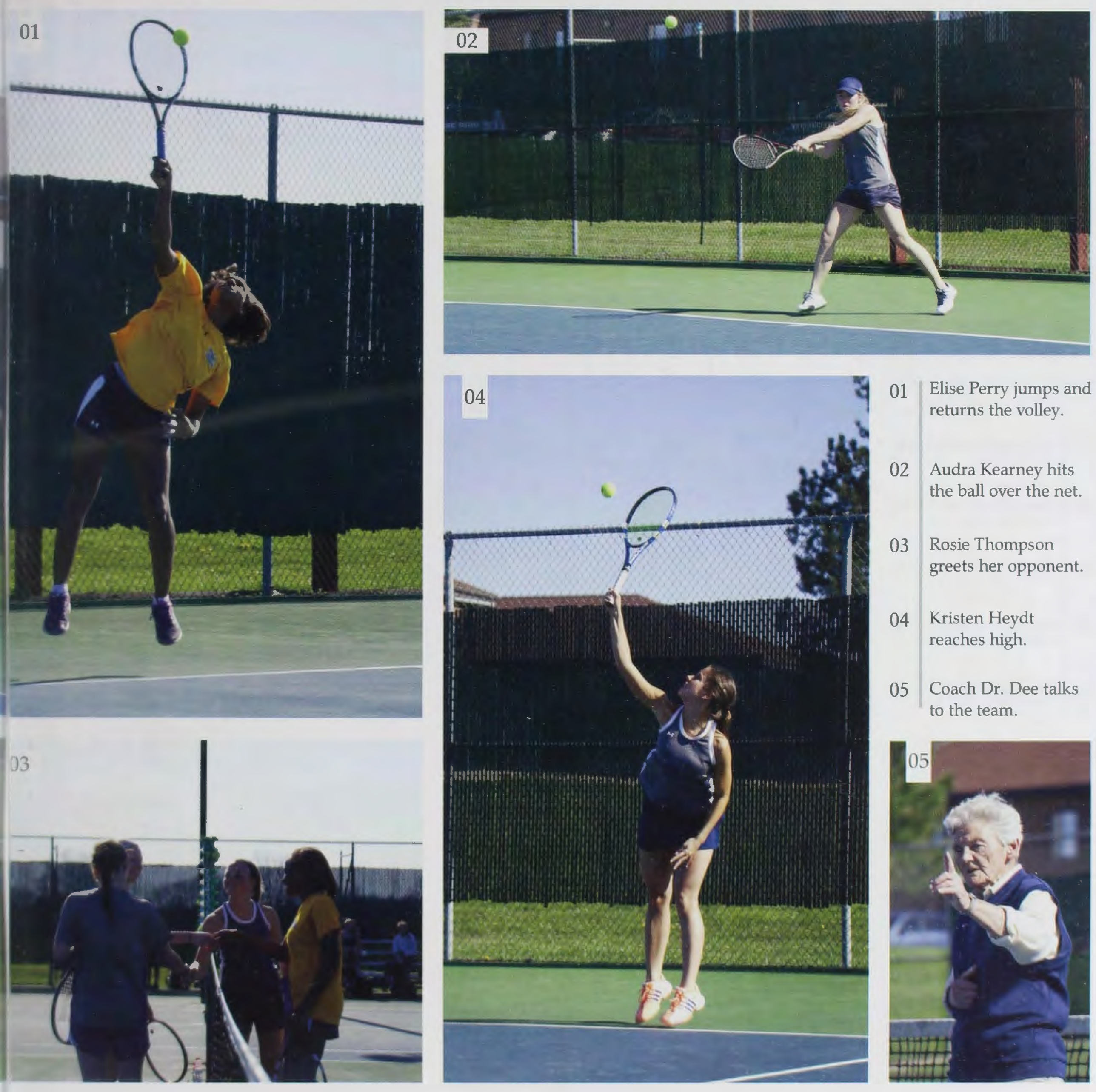

01 Elise Perry jumps and
returns the volley.

02

Audra Kearney hits the ball over the net.

03 Rosie Thompson greets her opponent.

04 Kristen Heydt reaches high.

05 Coach Dr. Dee talks to the team.

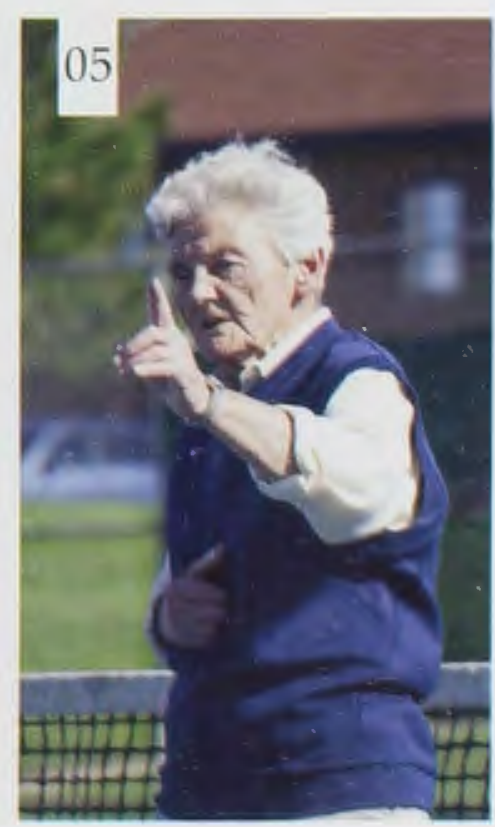

\section{Women's Tennis}

"Our time in Florida over spring break made us grow as a team. We had a night of sharing testimonies with each other, and it made us grow to understand each other's differences. I really loved that we came away from that trip so much closer and having more of a gospel outlook on it because of all the things Christ had done in our lives as individuals." 

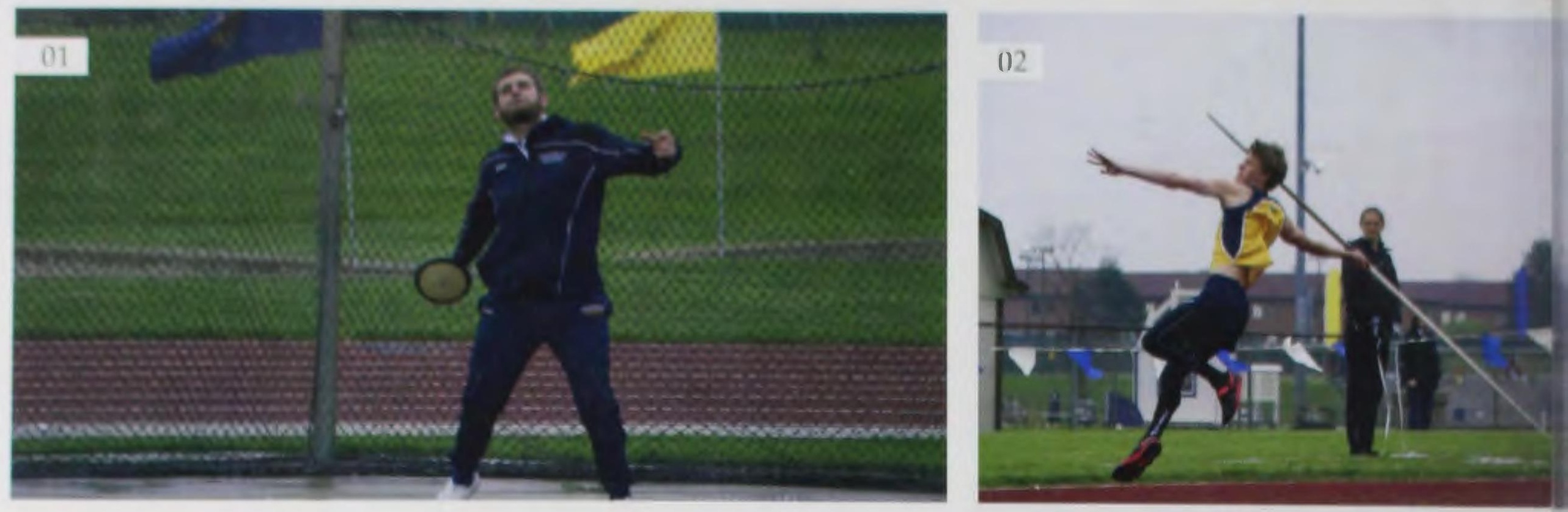

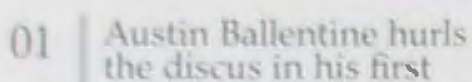

vear as a Yellow lacket

03

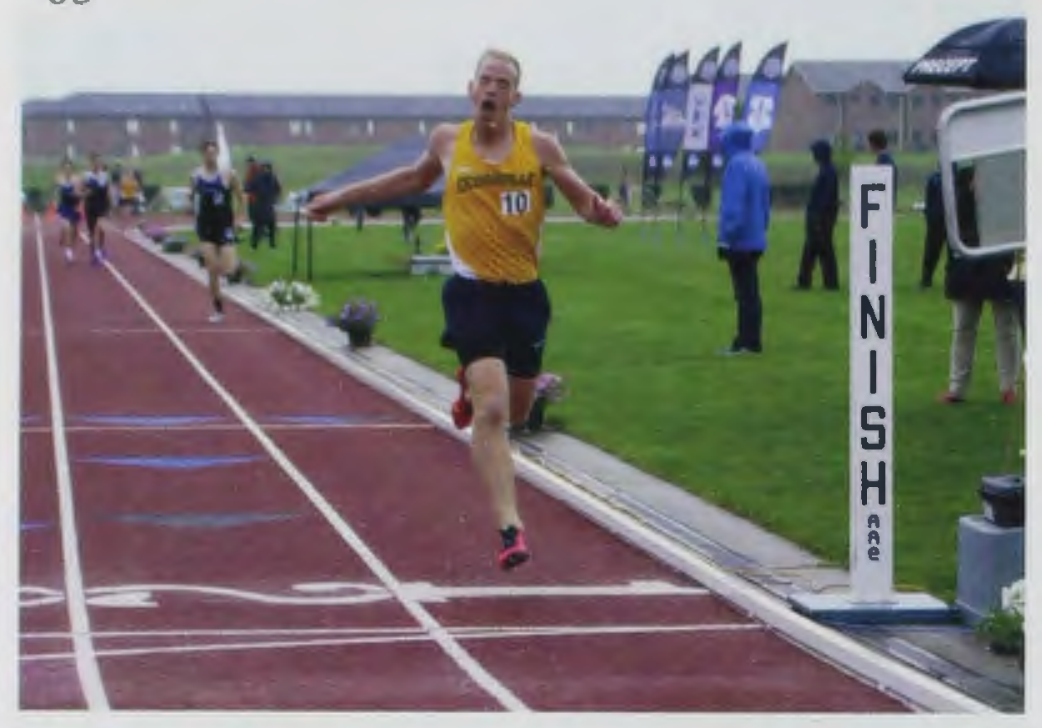

05

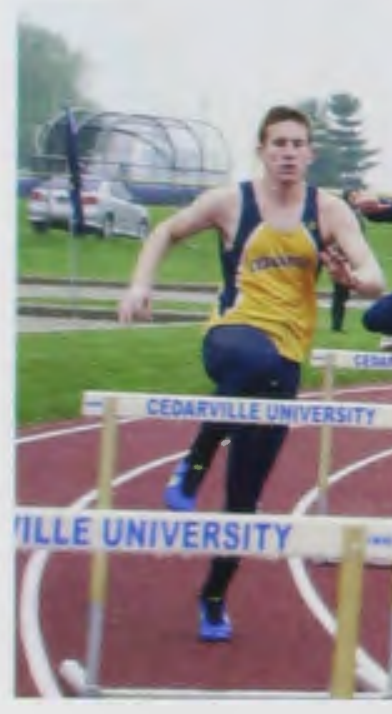

\section{Men's Track}

"The coaches pushed us harder in our daily practice, and our daily faith. For me, it's easy to forget the fun I can have with track and field, but having fellow Christian athletes who can alwats keep things fun and competitive is what draws me back every day." 


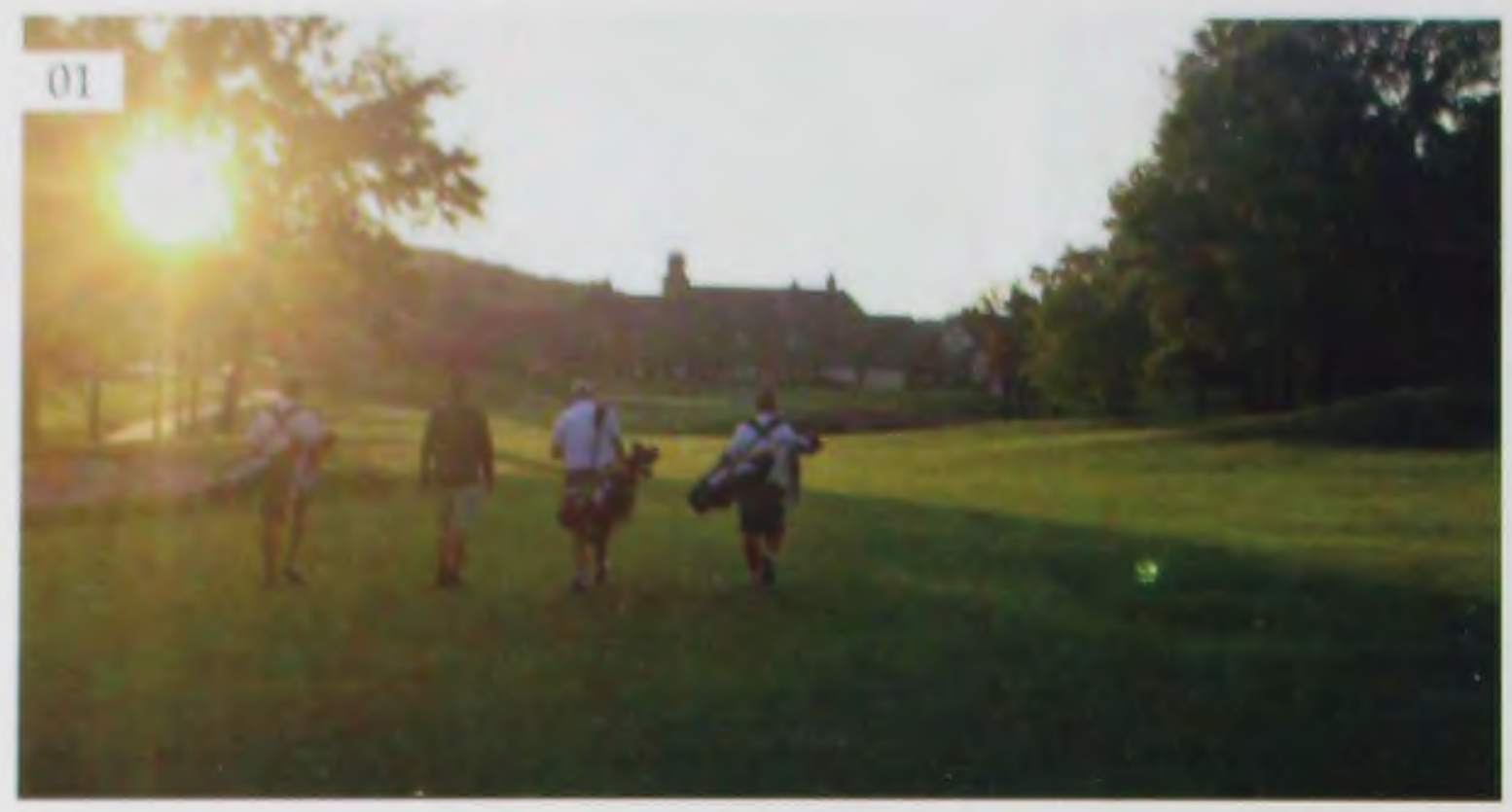

01 The team wraps up another round from their trip to Florida.

02 Jordan Glick powers a drive into the fairway

03 Taylor Holt watches his putt from the fringe intently.

04 Derek Truninger tees up an iron.

05

The Yellow Jackets warm-up on the range.
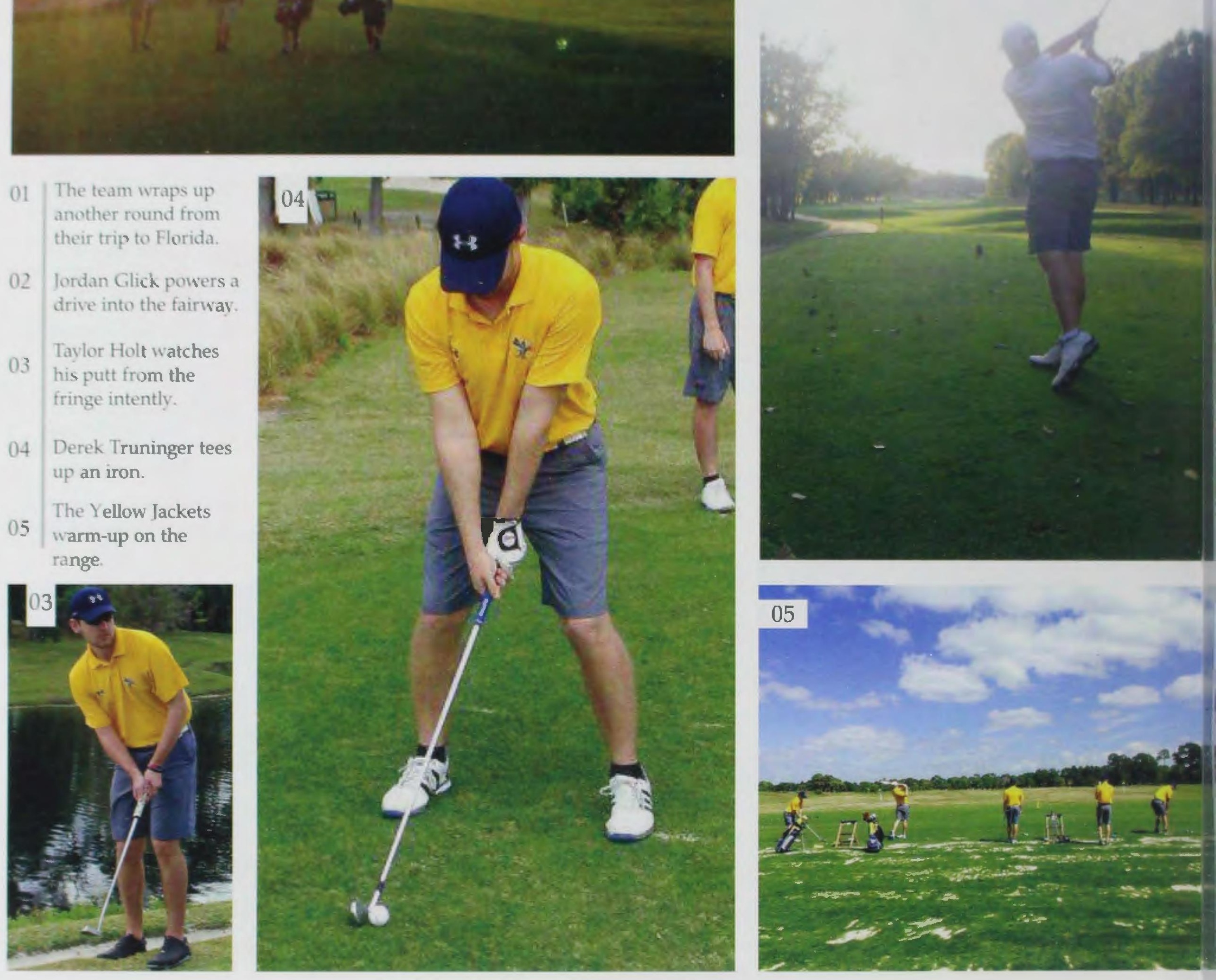

\section{Golf}

"The men's golf team had a strong fall season, finishing runner-up in two events and qualifying for the NCCAA National Championship in Panama City, Florida. Our spring season was highlighted by a second place finish at the G-MAC Championship. Sophomore Jordan Reese and junior Taylor Holt were selected to G-MAC All Conference Team." 

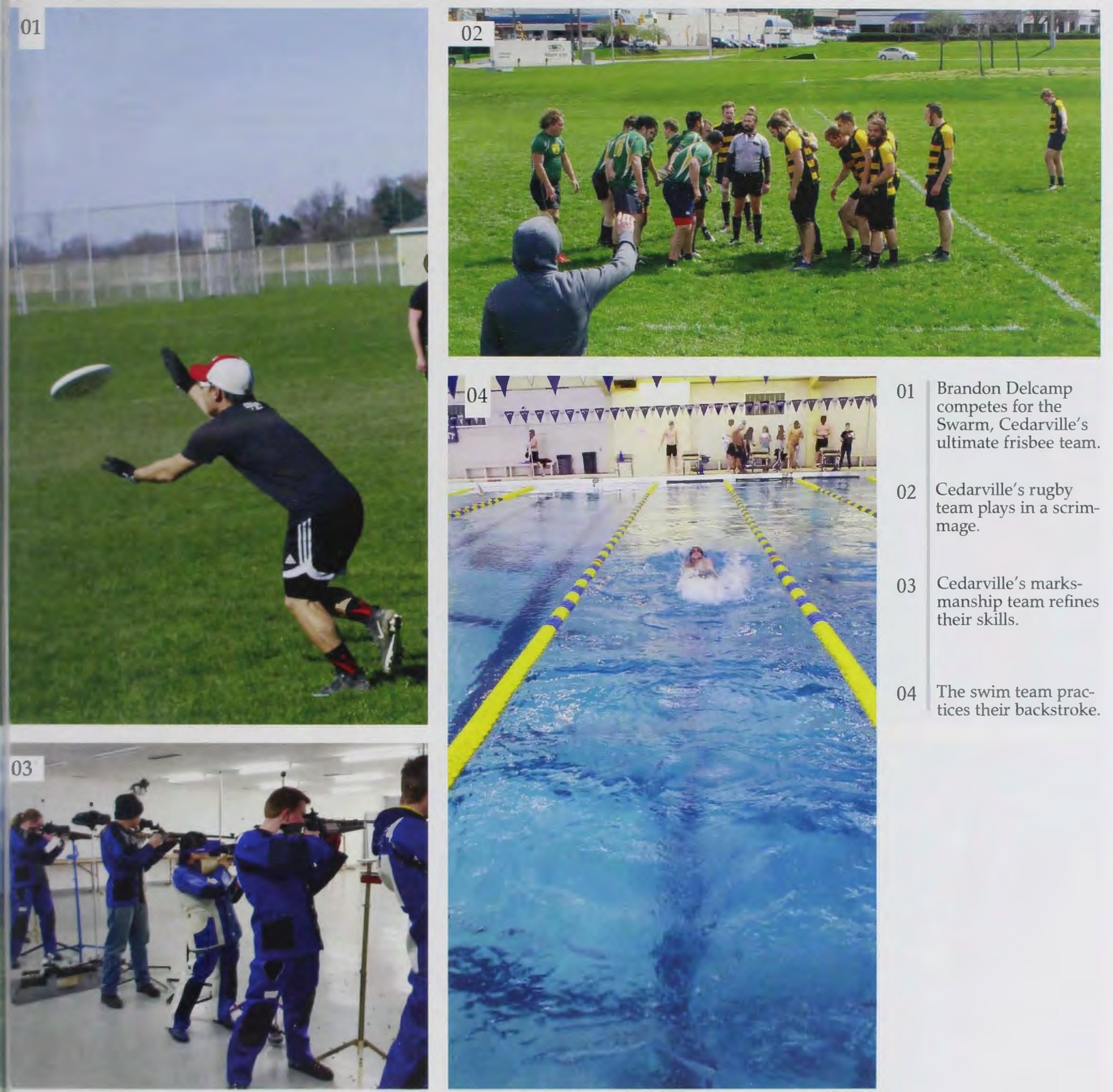

01 Brandon Delcamp competes for the Swarm, Cedarville's ultimate frisbee team.

02 Cedarville's rugby team plays in a scrimmage.

Cedarville's marksmanship team refines their skills.

04 The swim team practices their backstroke.

\section{Club Sports}

"I had an amazing time being able to play ultimate frisbee this year. It's such a blessing playing with such talented and handsome guys. The friendship and bonds we've created together are unbreakable. To be able to be a witness for Christ on the field is a great privilege." 

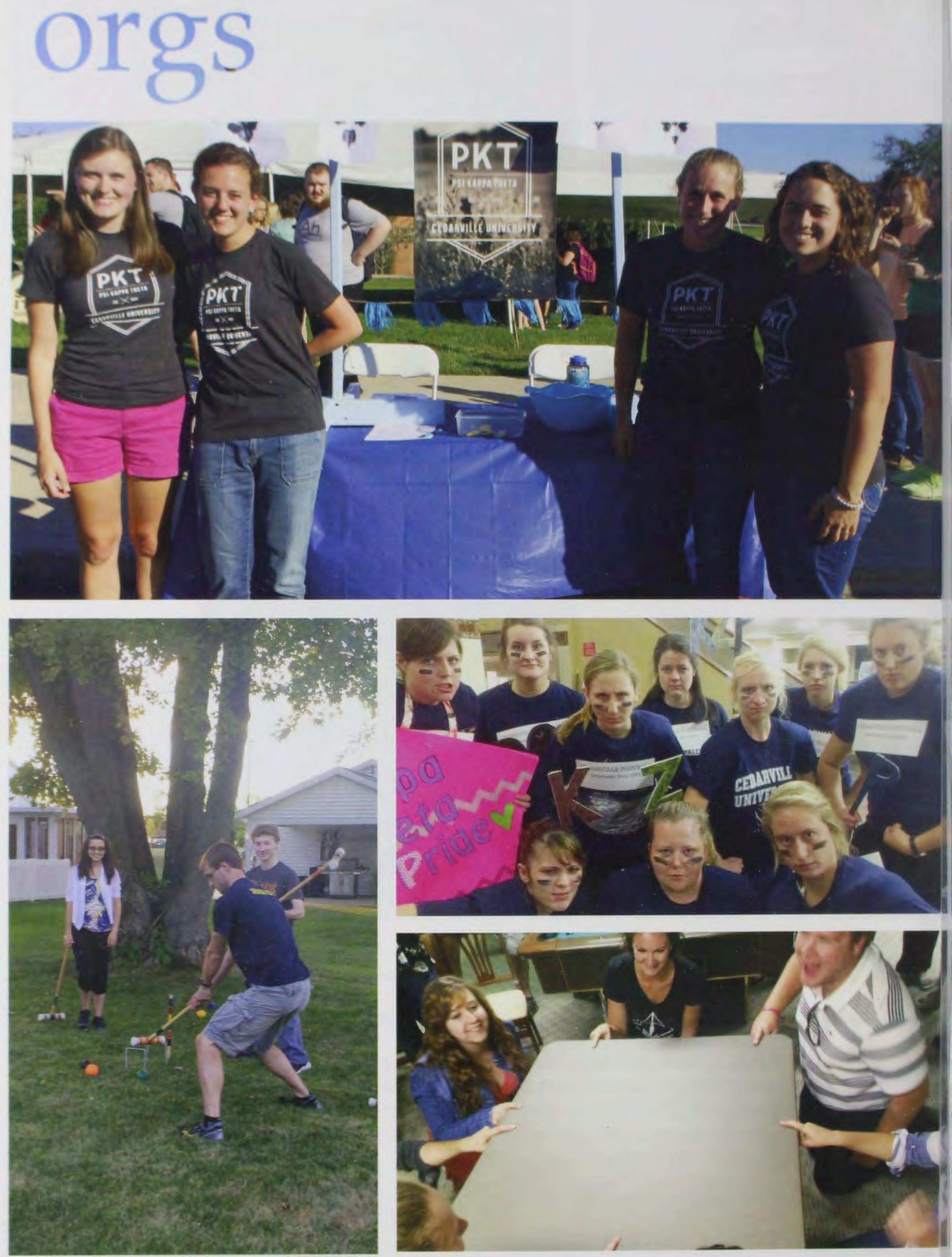

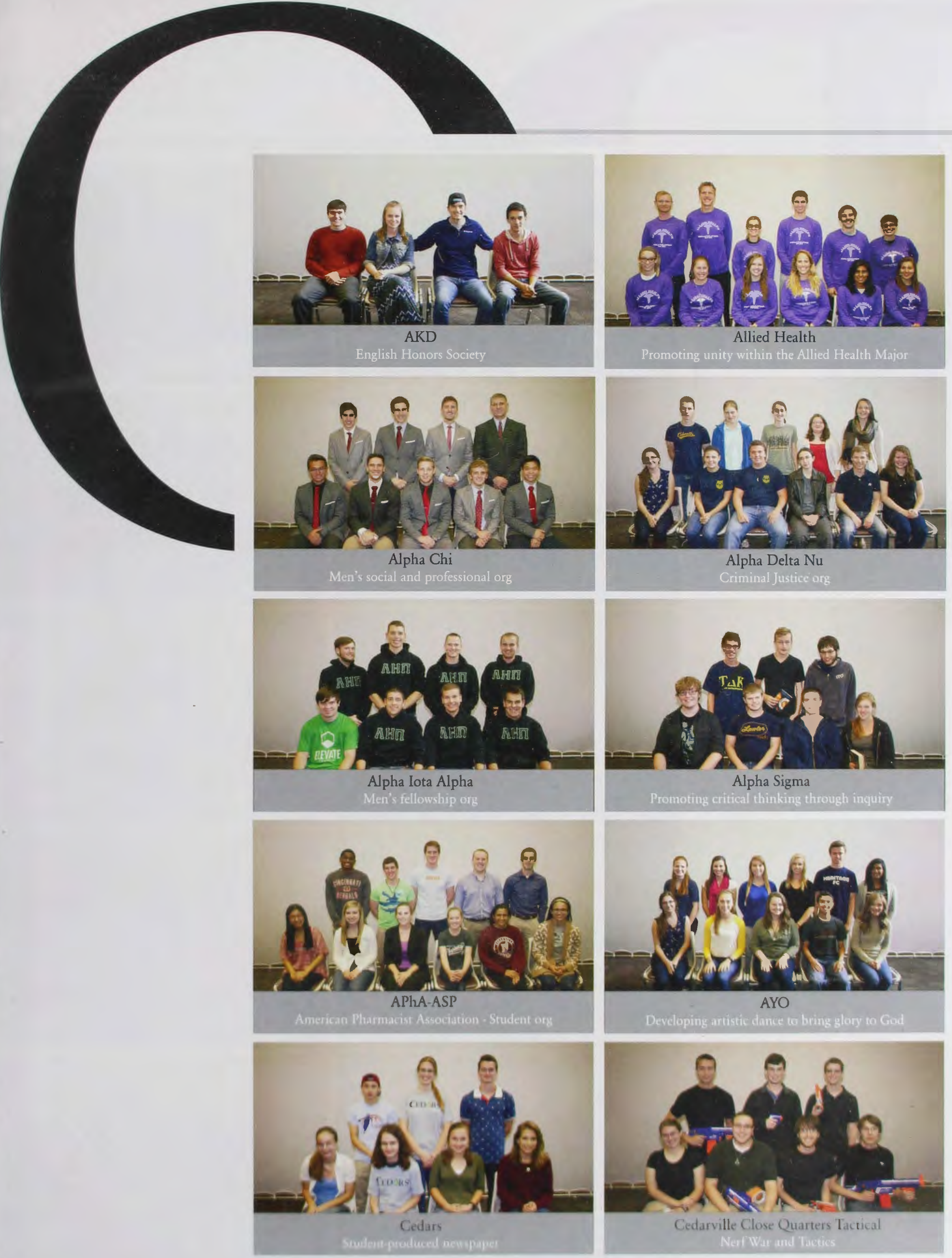

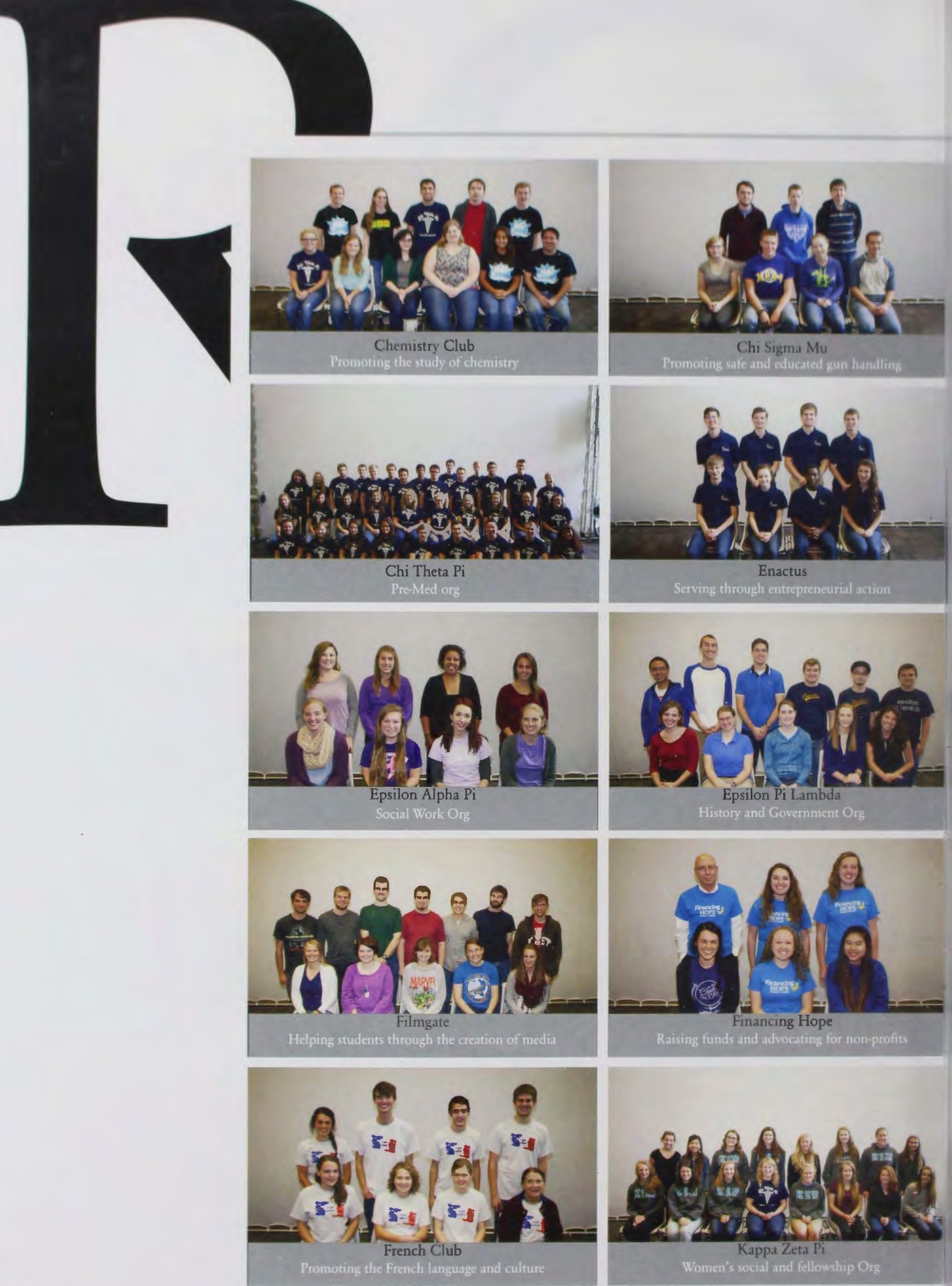

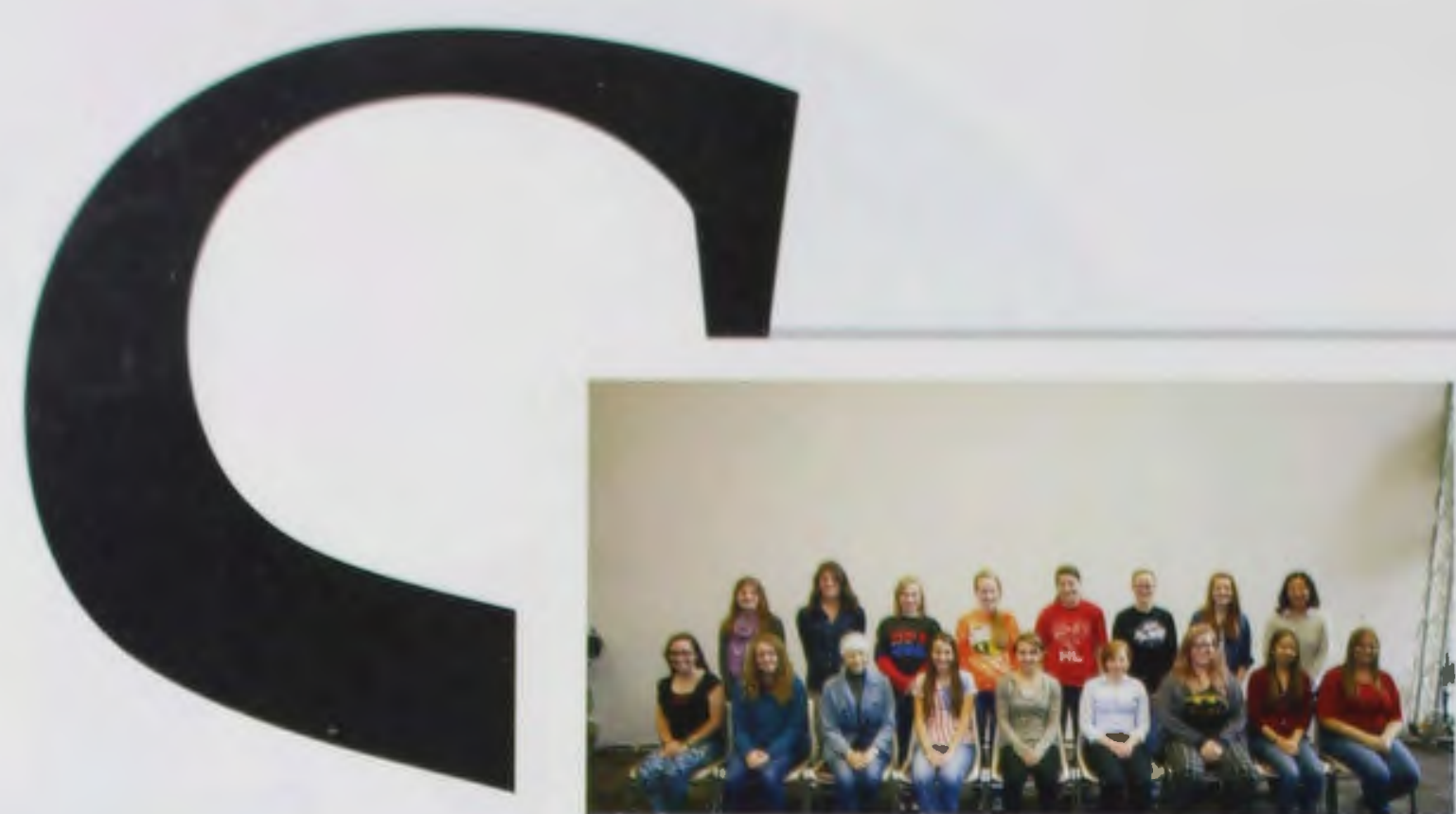

Society of Women Engineers

Women engineers community and service org
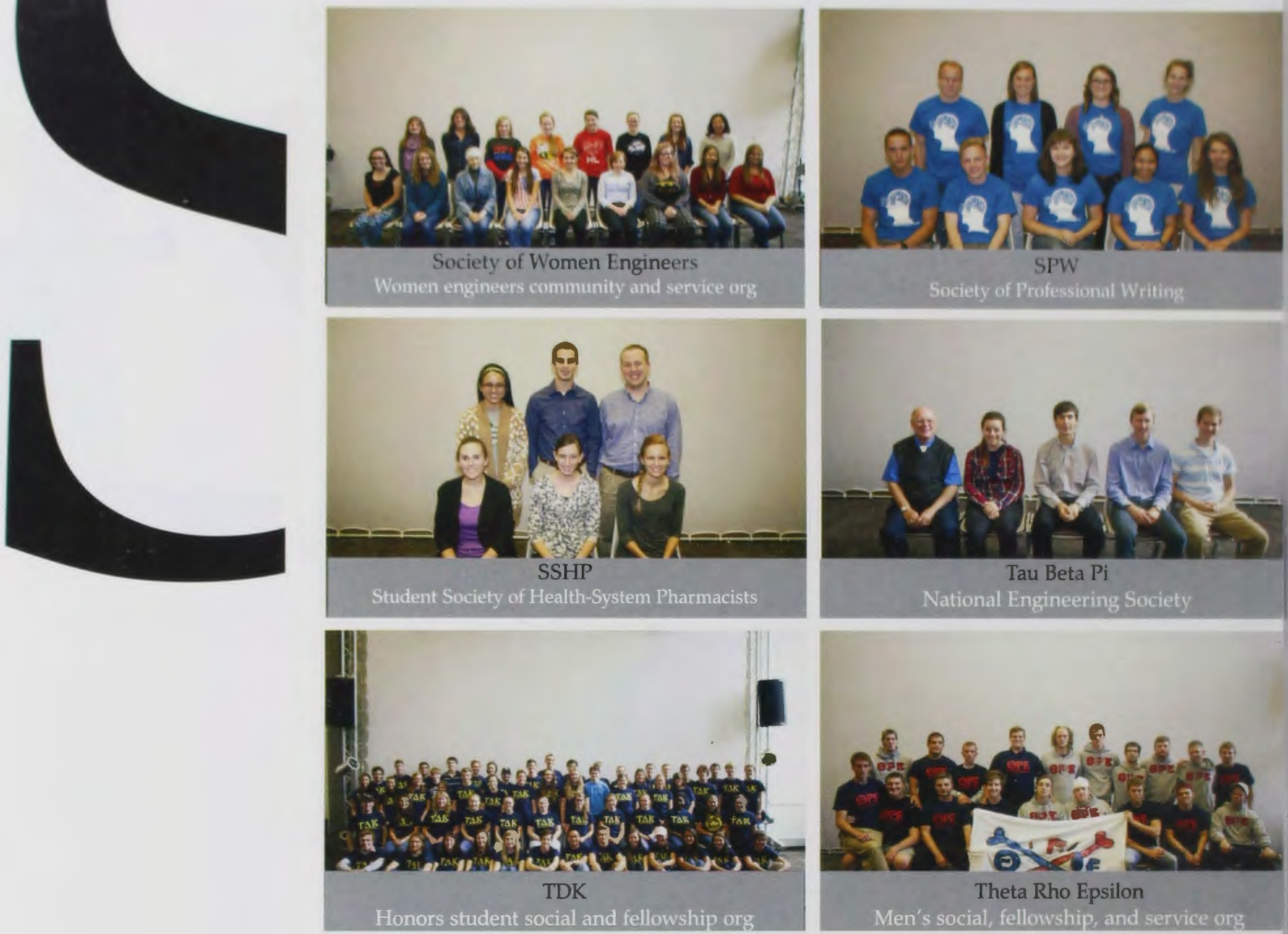

Theta Rho Epsilon

Men's social, fellowship, and service org

"There is nothing in this world that can compare with the Christian fellowship; nothing that can satisfy but Christ." 


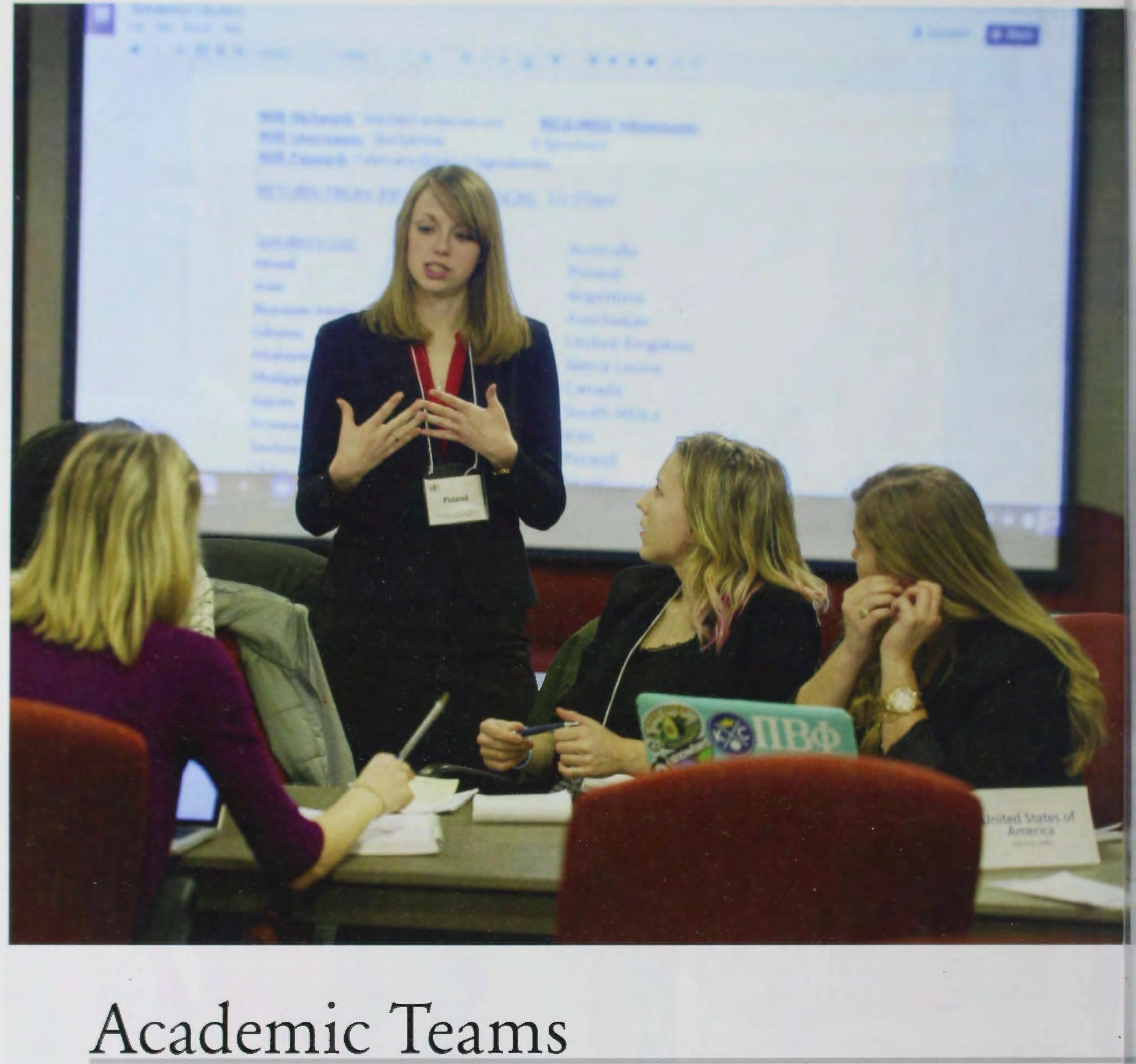

As a Christ-centered learning community, Cedarville is committed to cultivating the mind and utilizing the intellect to serve and glorify God. One area where students could practice this outside the classroom was through academic teams. Groups such as Forensics, Model UN, Debate, and Supermileage travelled across the country as representatives of Cedarville and of Christ, using their talents to create, present, and perform in various regional and national competitions.
Led by experienced professors and advisors, these student "athletes of the mind" proved they could think deeply, communicate effectively, develop academically and professionally, engage for Christ, and glorify God. 
Faculty and Staff

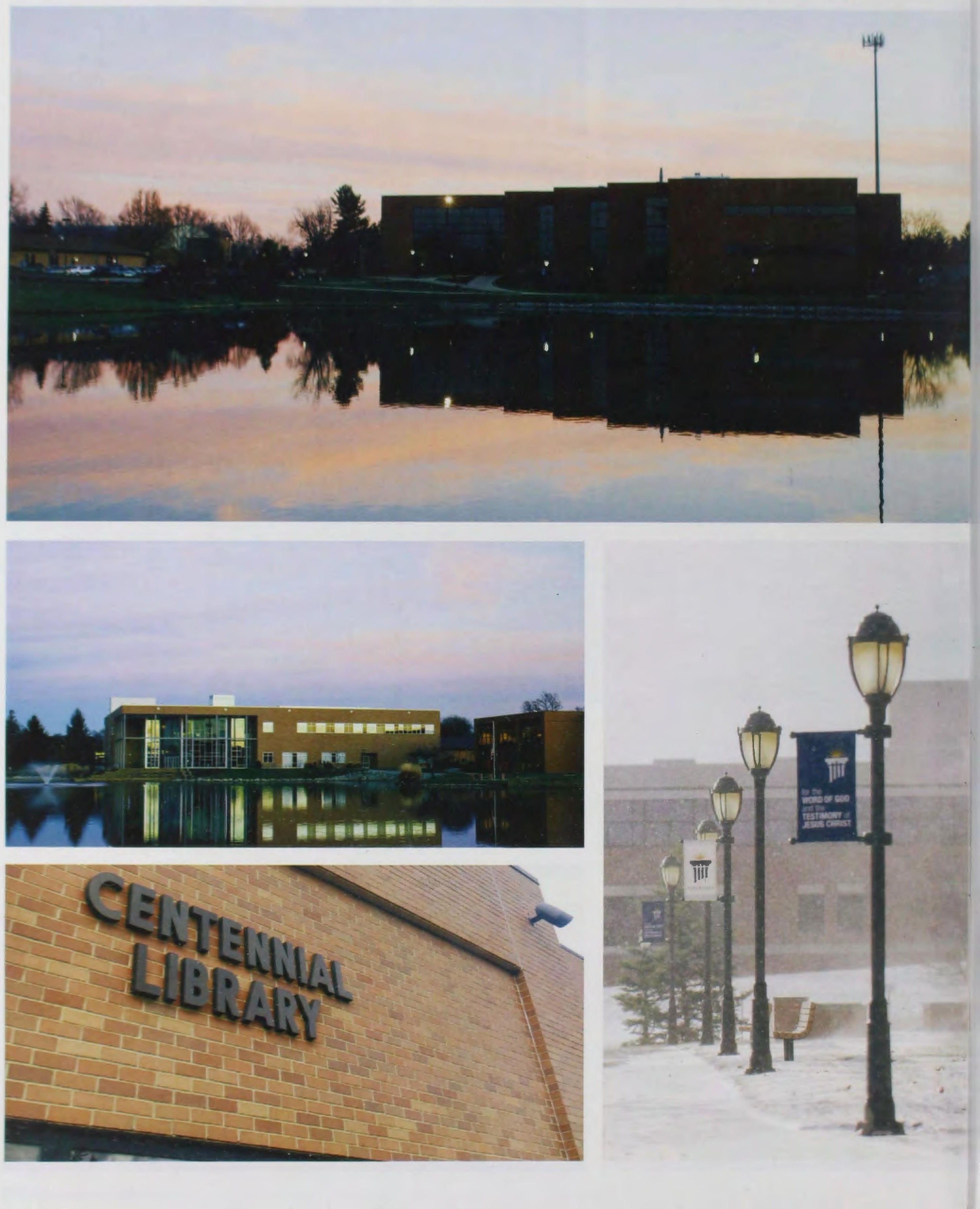




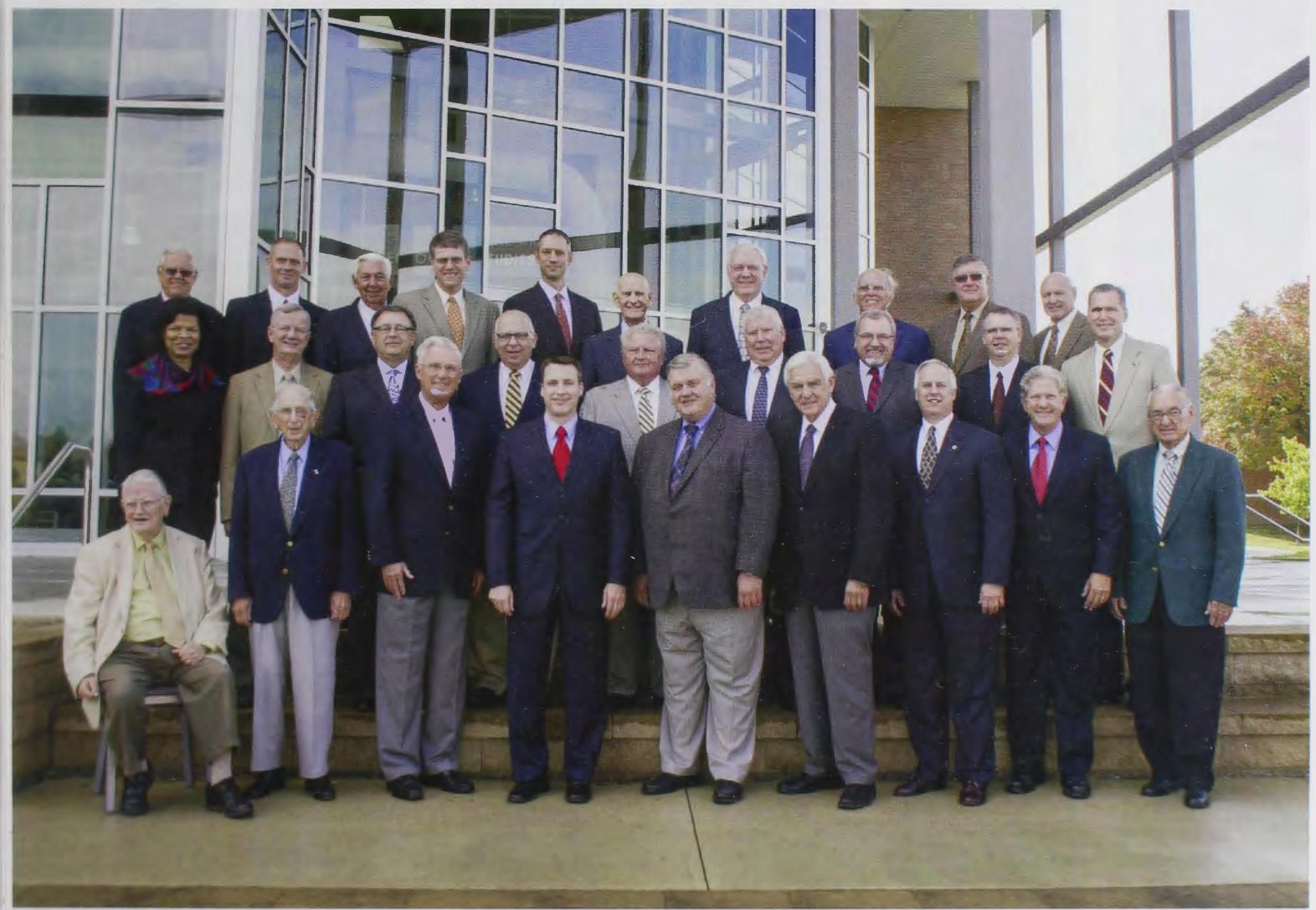

\section{Trustees}

Corey Abney, Daniel Akin, Timothy Armstrong, Chip Bernhard Jr.

John Blodget, David Carr, Dave Dykema, Evan English, David Gower, David Graham, Gina Headrick, Warren Jenkins, Deforia Lane, David Lodwick, Don Lough Jr, Dominic McKinley, Randy Patterson, Paige Patterson, Dan Petek, Lorne Scharnberg, Mark Vroegop, Dave Warren, Hayes Wicker, Jeff Willetts, Rob Wynalda Jr., Bill Bolthouse 
Faculty \& Staff

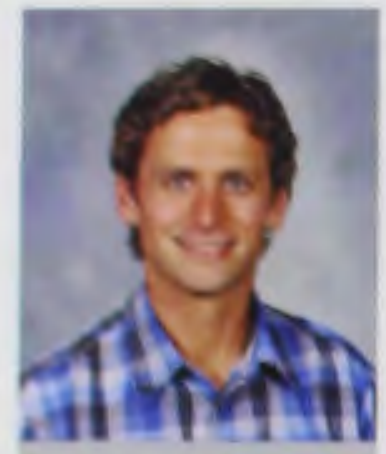

Chris Bruno

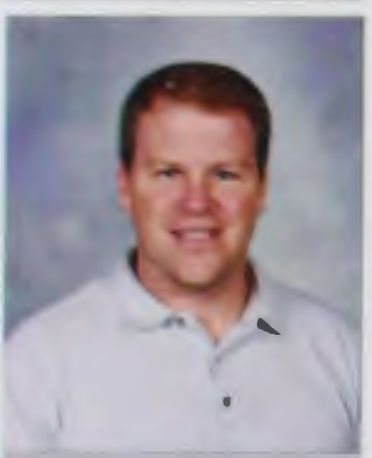

Andrew Wonders

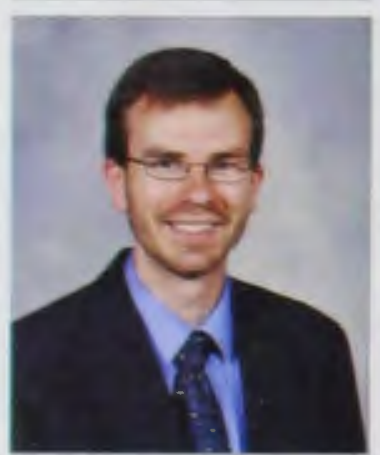

Patrick Dudenhofer

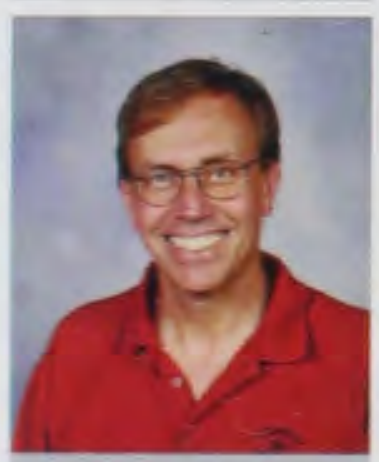

Jay Kinsinger

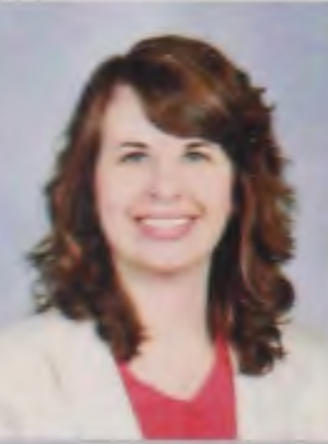

Sarah Murphy

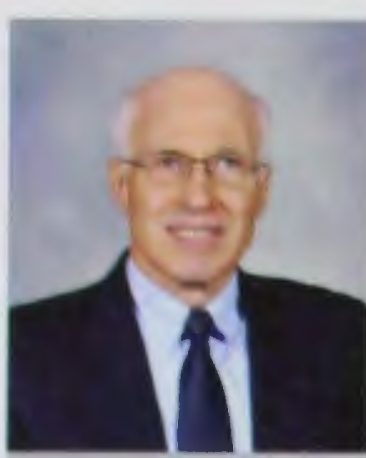

Dan Fstes

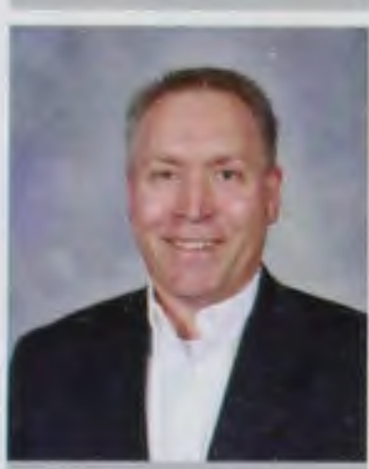

Jeremy Ervin

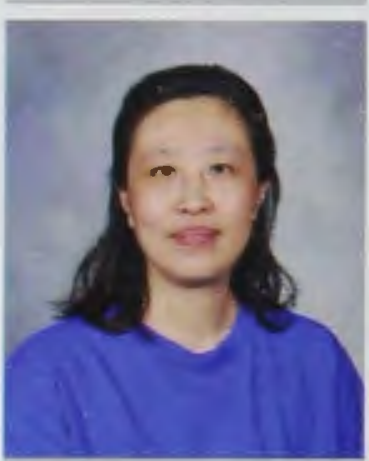

Vicky Fang

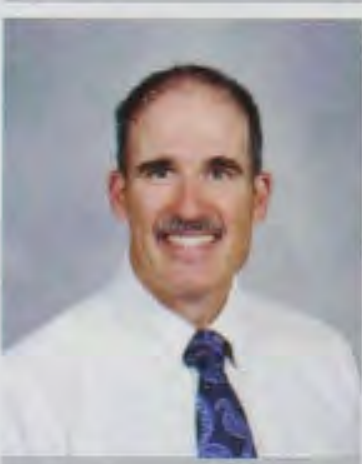

Keith Shomper

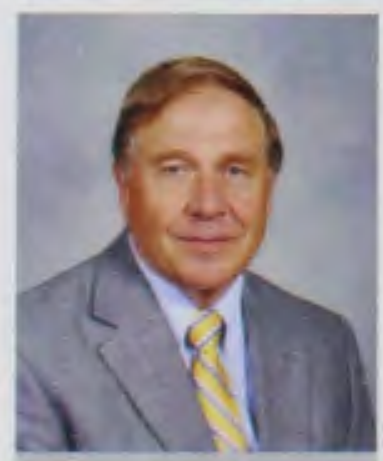

Jim Phipps

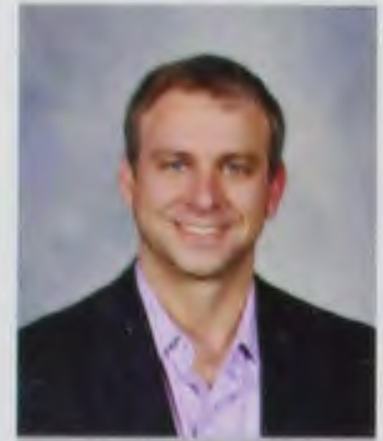

William Marsh

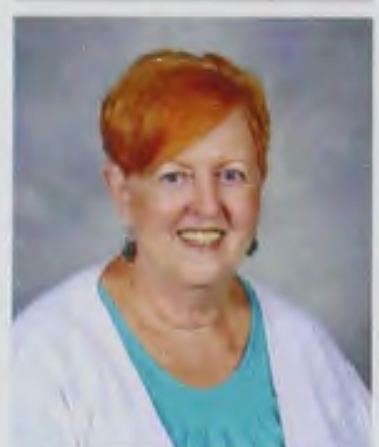

Brenda MacKay

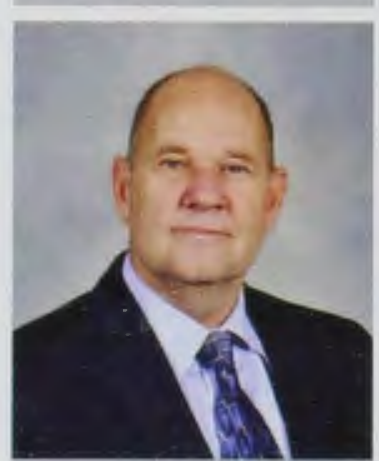

David Gallagher

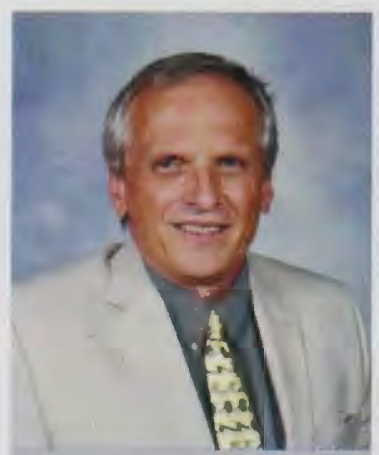

Jeff Shortt

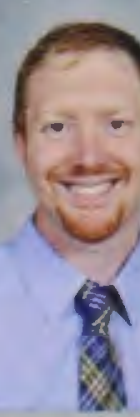

Jeff Simon

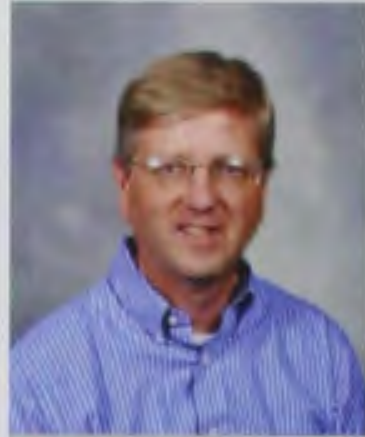

Joel Williams

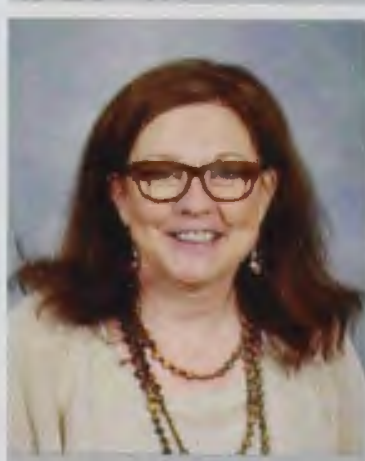

Lynn Roper

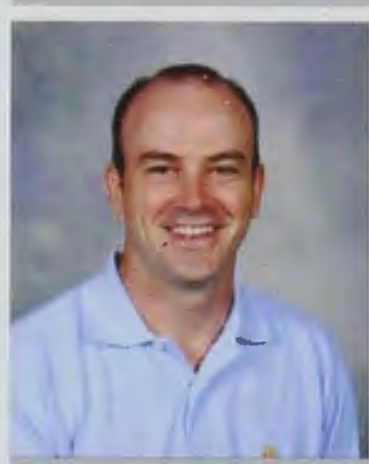

Seth Hamman

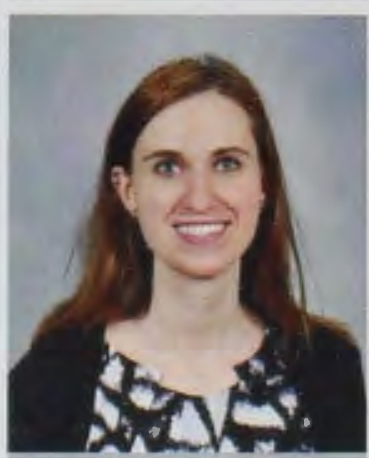

Aleda Chen

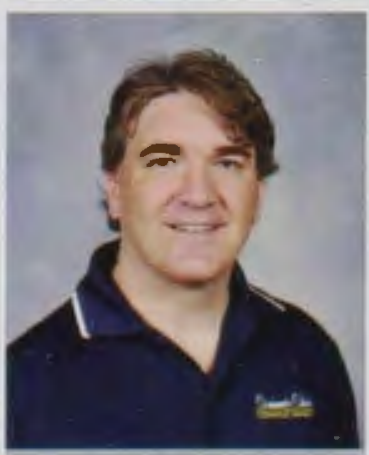

Donald Deardorff

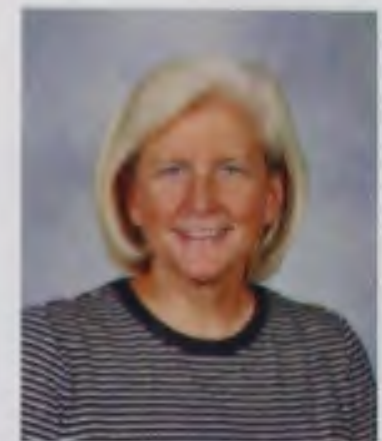

Sue Terkelsen

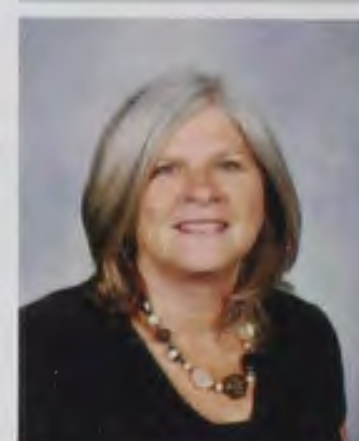

Ruth Sylvester

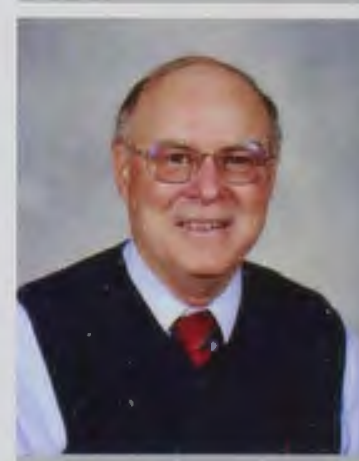

Hardy Hegna

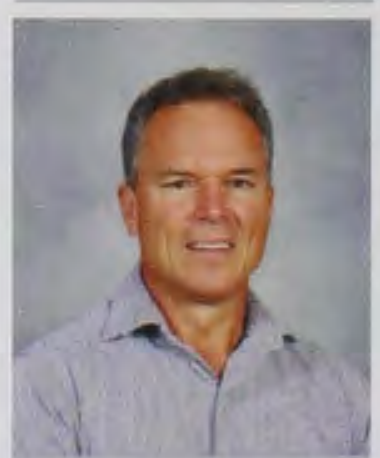

Rocco Rotello

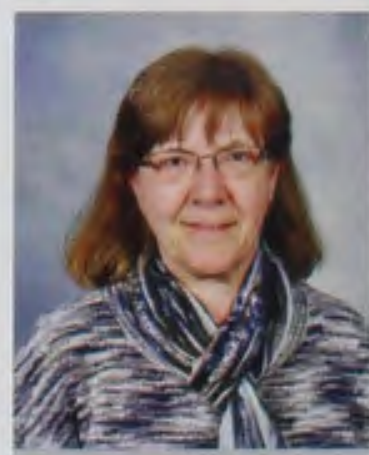

Barbara Loach

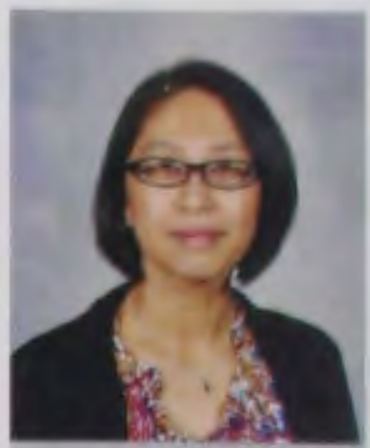

Phoebe Tsai

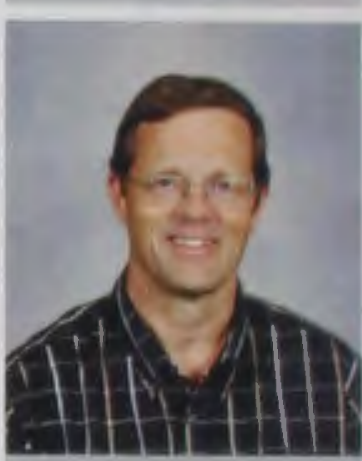

Gerald Brown

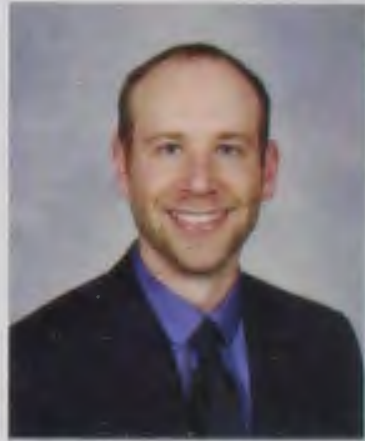

Darren Holland

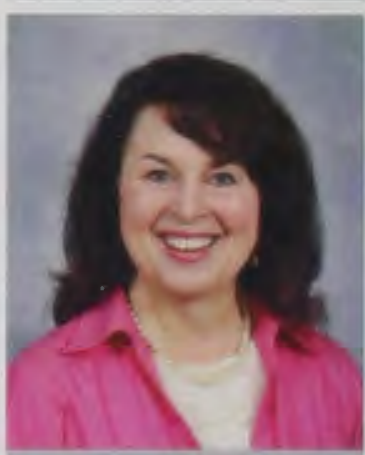

Rebecca Baker

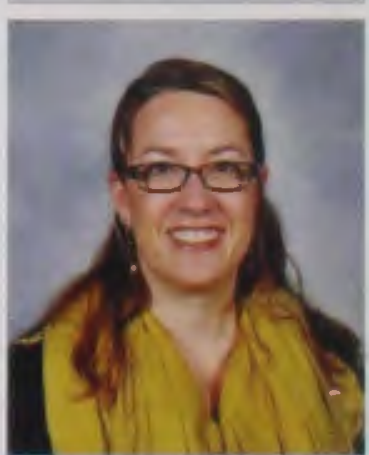

Cyndi Messer 


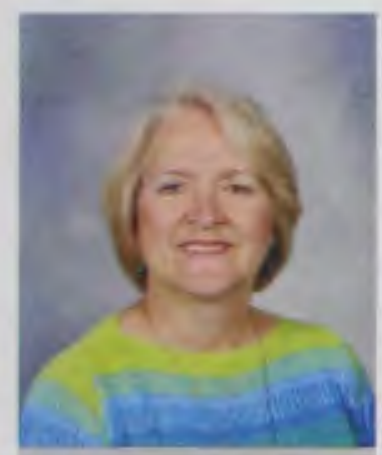

Annis Shaver

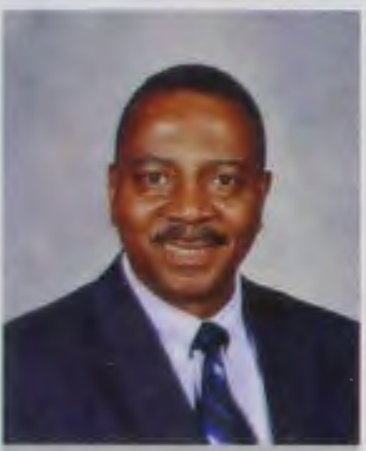

Patrick Oliver

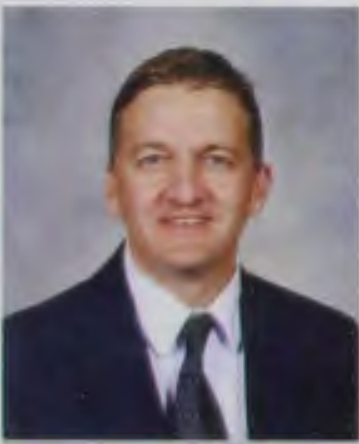

Robert Vaughn

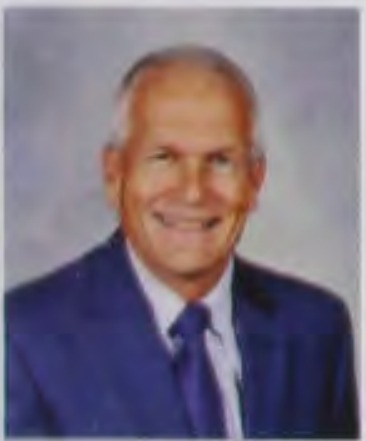

John MeGillivray

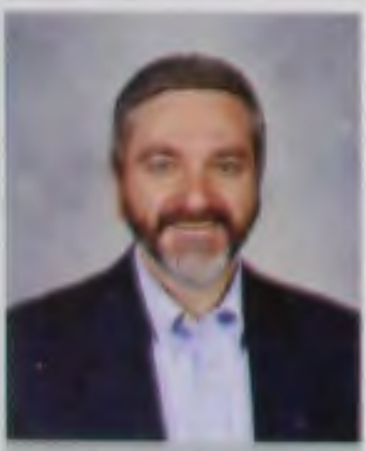

Michael Firmin

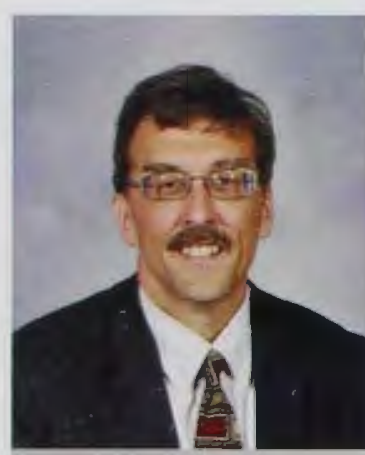

Marc Clauson

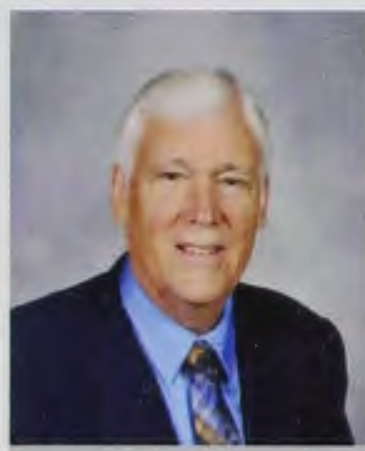

Bob Parr

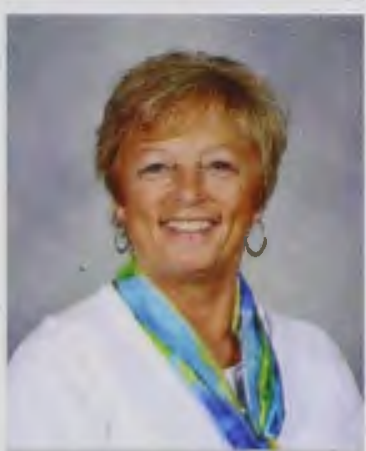

Teresa Clark

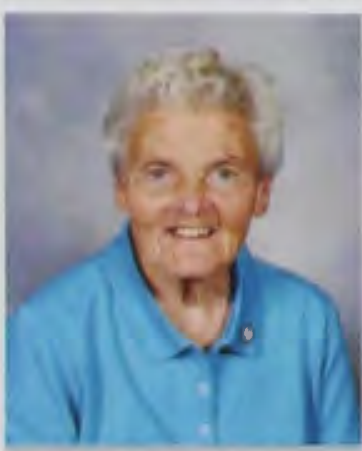

Dee Morris

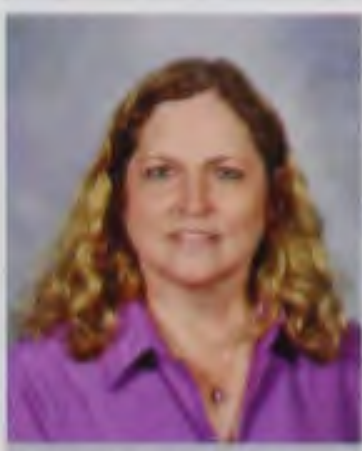

Ruth Markham

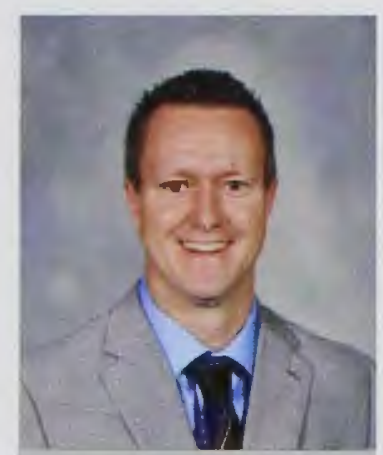

Glen Duerr

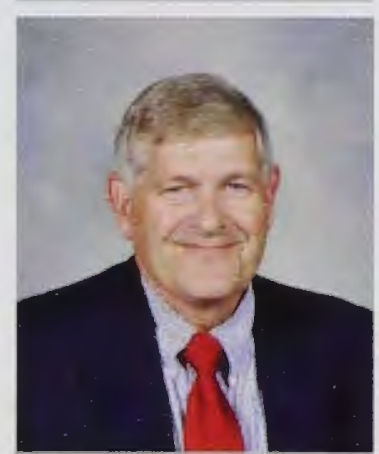

David Rich

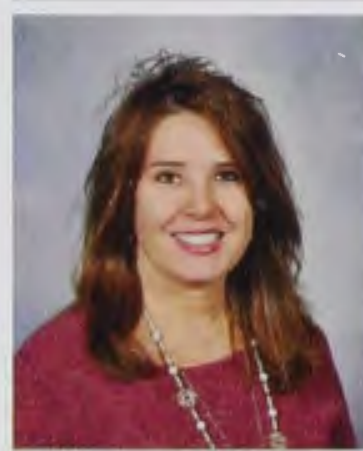

April Crommett

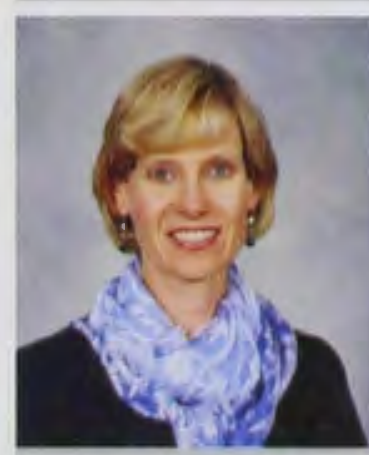

Elizabeth Sled

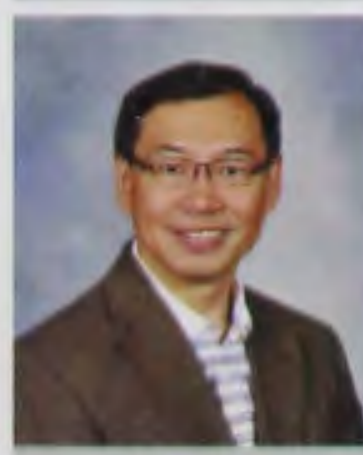

Luke Tse

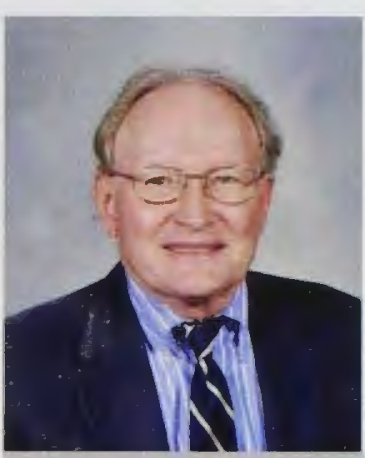

Frank Jenista

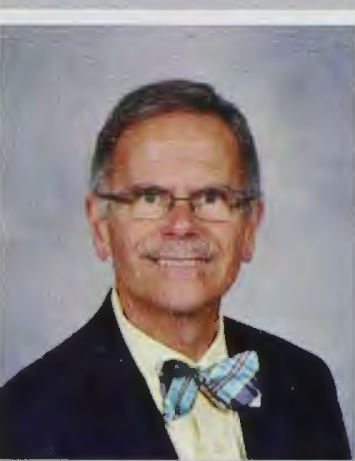

Kevin Sims

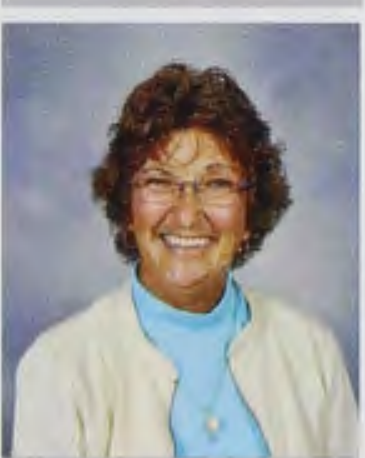

Kathy Freese

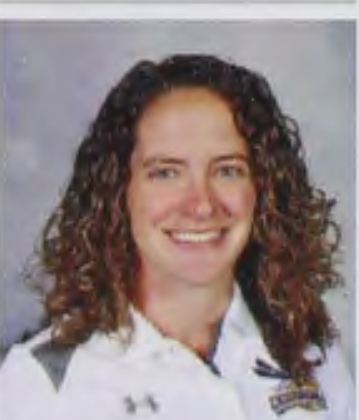

Hannah Stedge

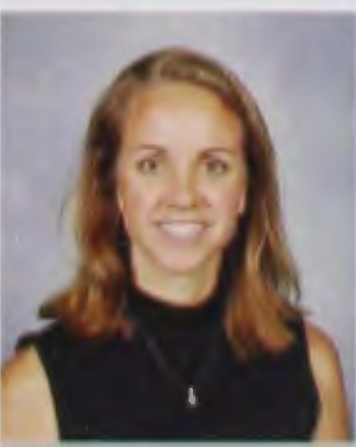

Melissa Burns

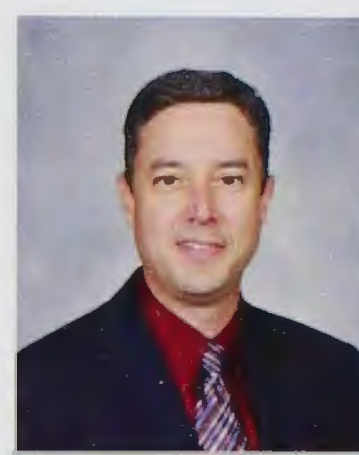

Tom Mach

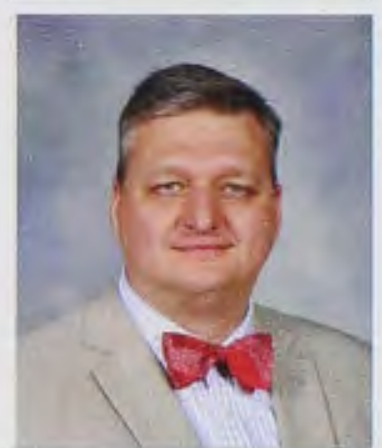

Mark Smith

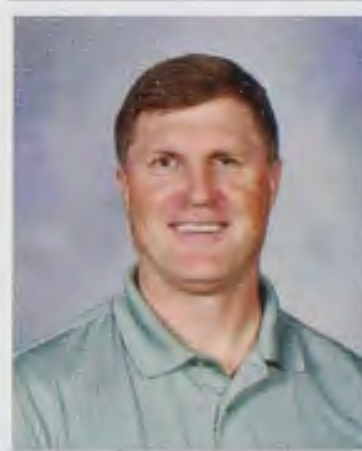

Evan Hellwig

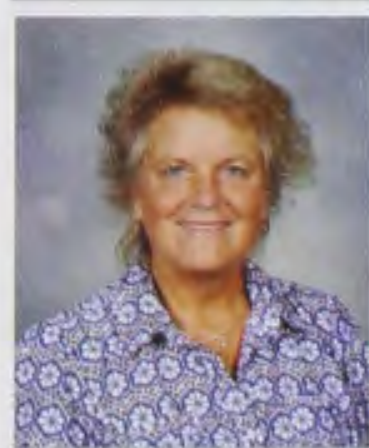

Kristin DeWitt

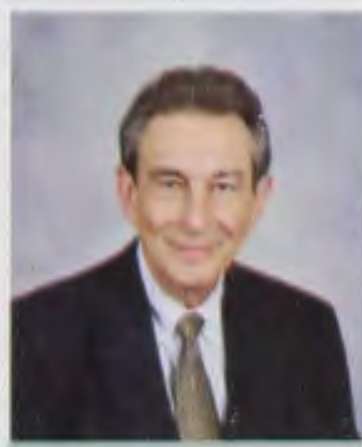

Dunnis Flentge

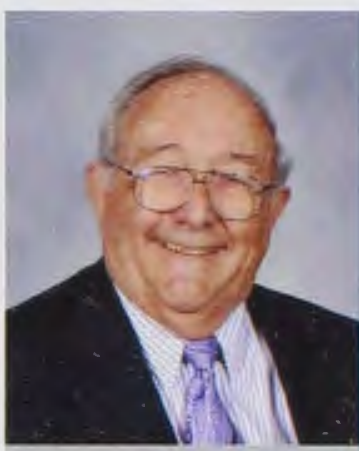

Murray Murdoch

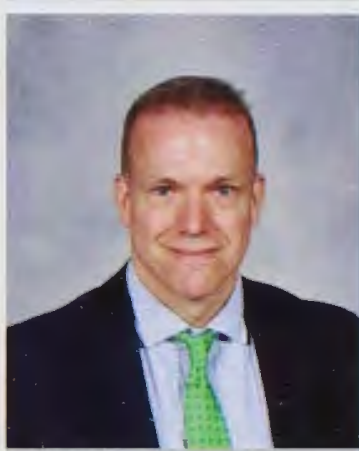

Rick Tison

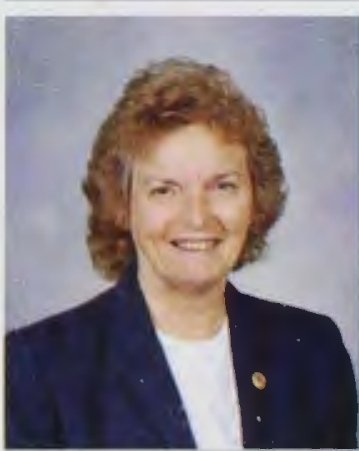

Pam Johnson

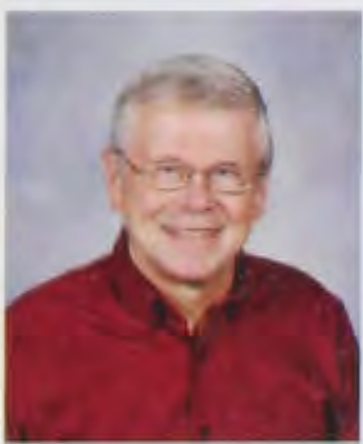

Chuck Dolph

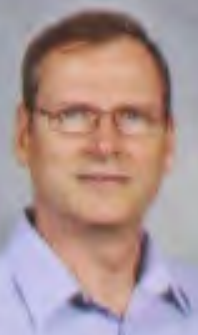




\section{Faculty \& Staff}

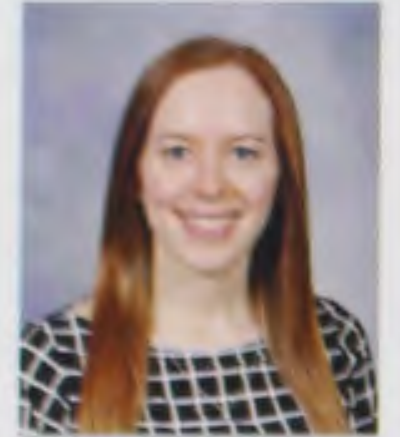

Ashley Holland

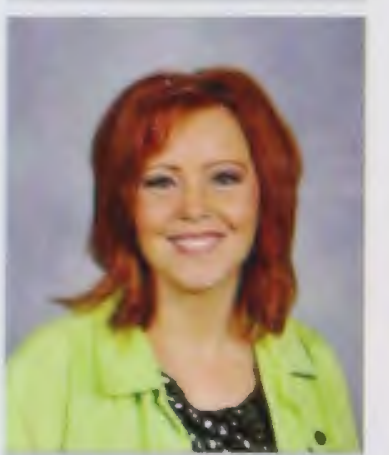

Melissa Brown

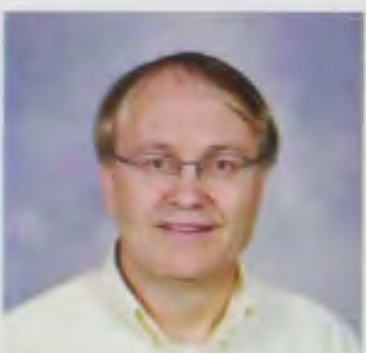

Jeff Gates

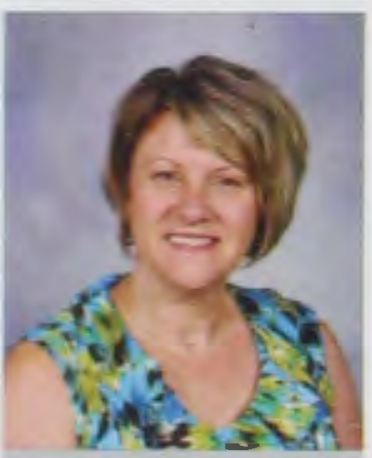

Sue Beikert

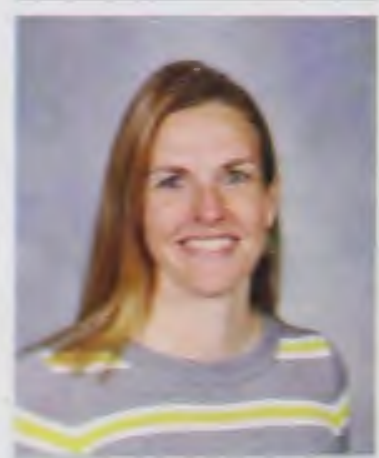

Tricia Clark

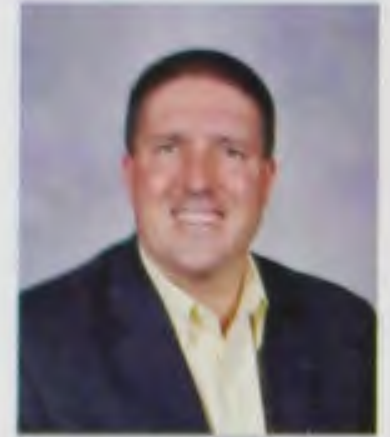

Geoff Holloway

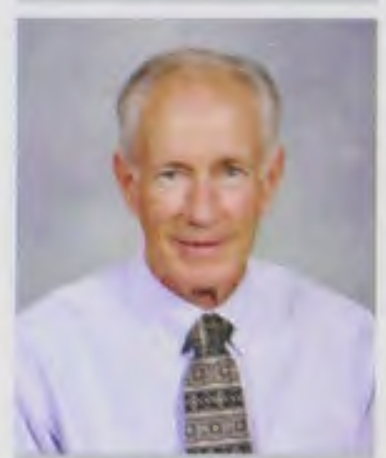

George Huff

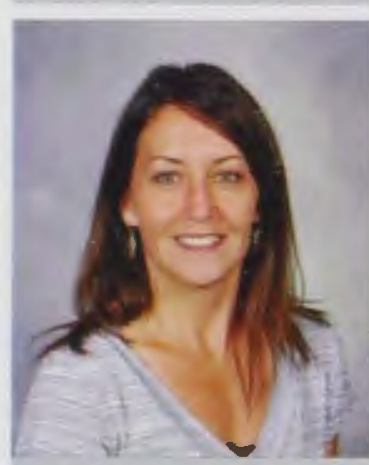

Sharon Kerestes

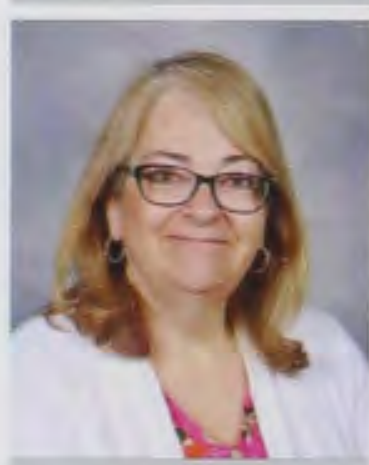

Âmy Bielek

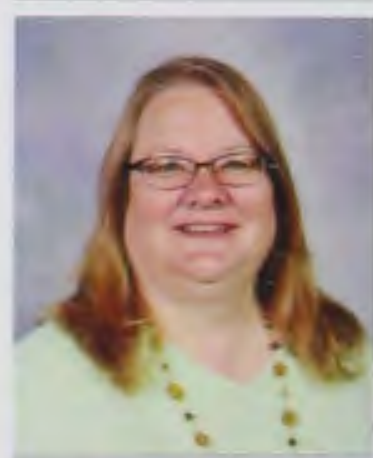

Renee Coffman

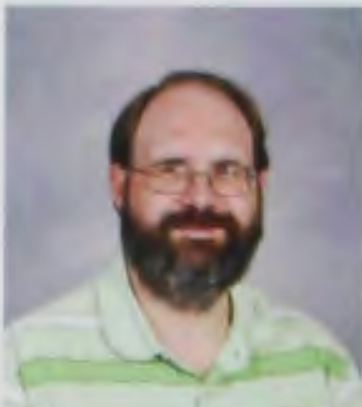

Aaron Hutchison

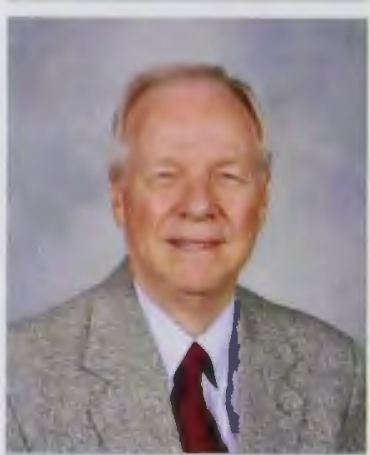

Lynn Brock

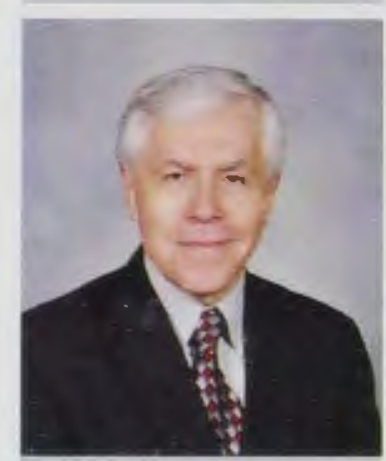

Gregory Martin

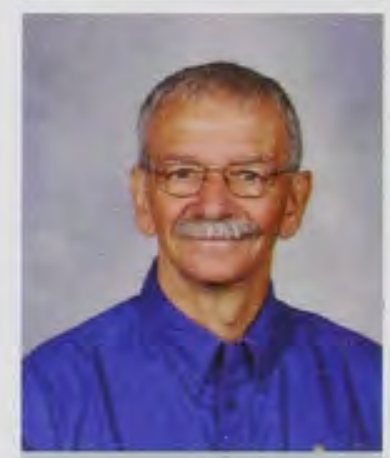

Robert Bielek

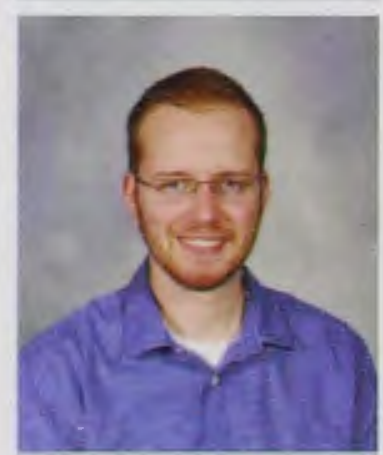

Luke Comers

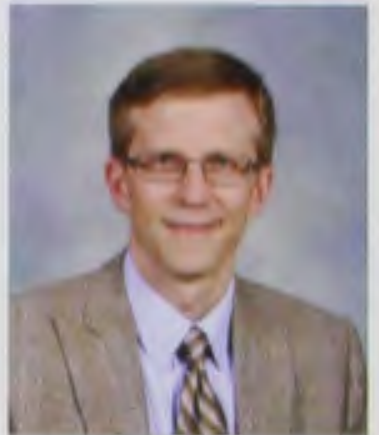

Mark McClain

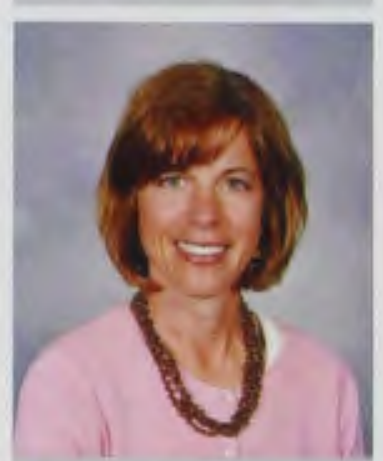

Kristi Coe

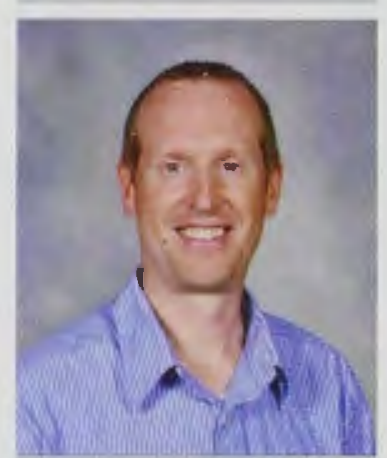

Josh Michael

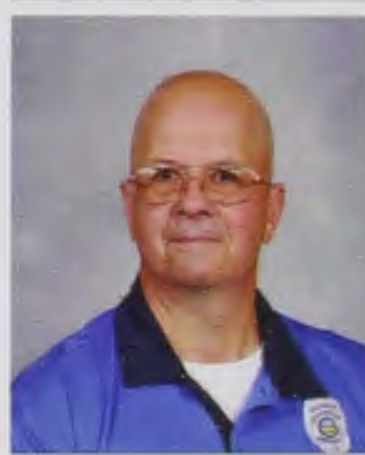

James Bowersox

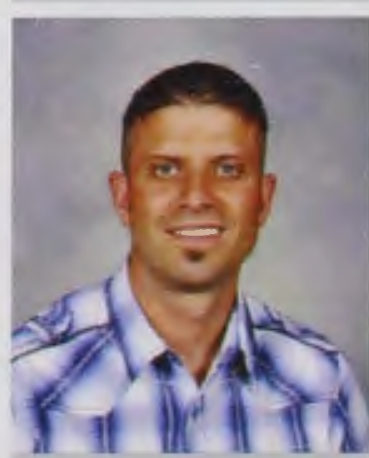

Aaron Cook

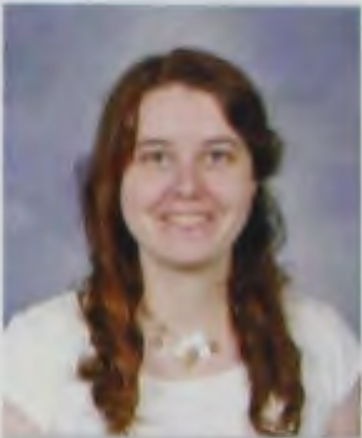

Nina Sekerak

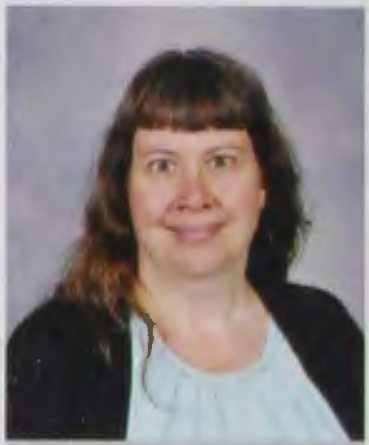

Julie Deardorff

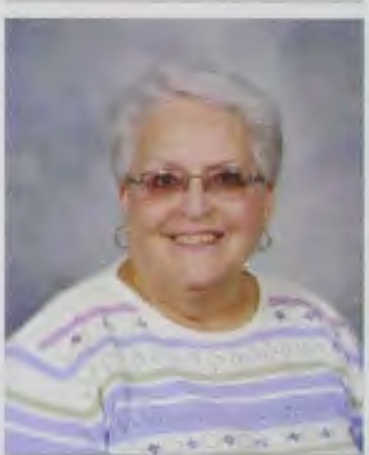

Michal Bader

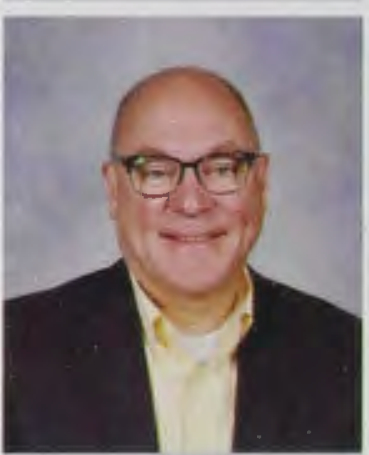

Jim Cato

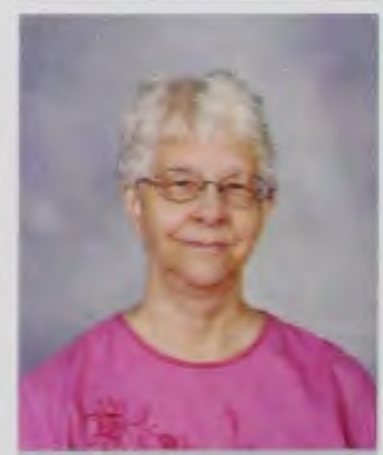

Linda Divan

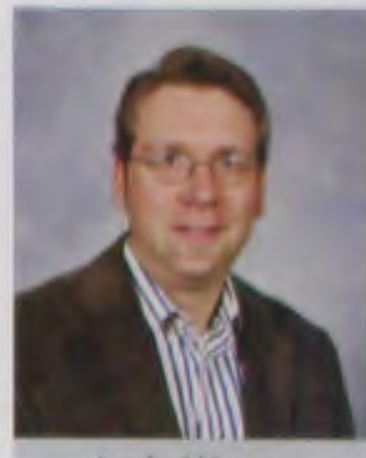

Andy Wagers

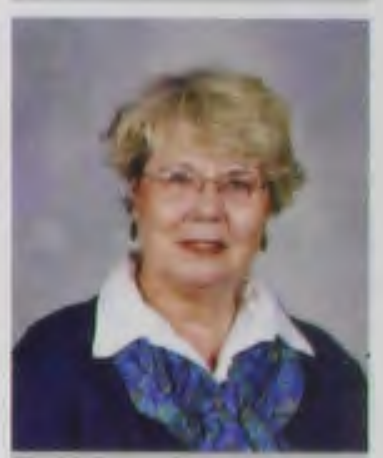

Lynne Funtik

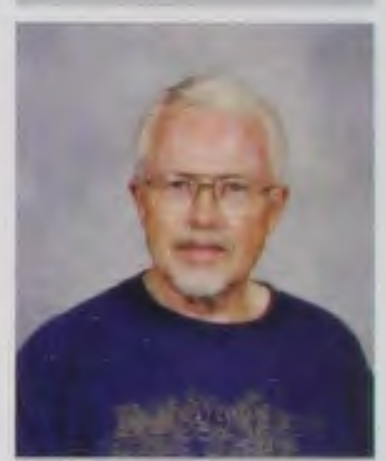

Ron Bader

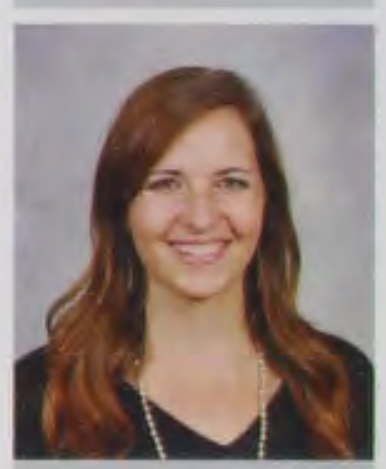

Rebekah Ciccart:llo

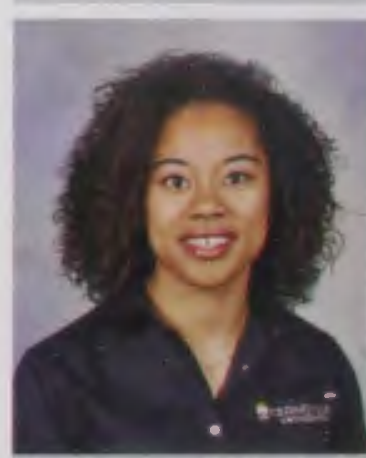

Jessi Dyson 


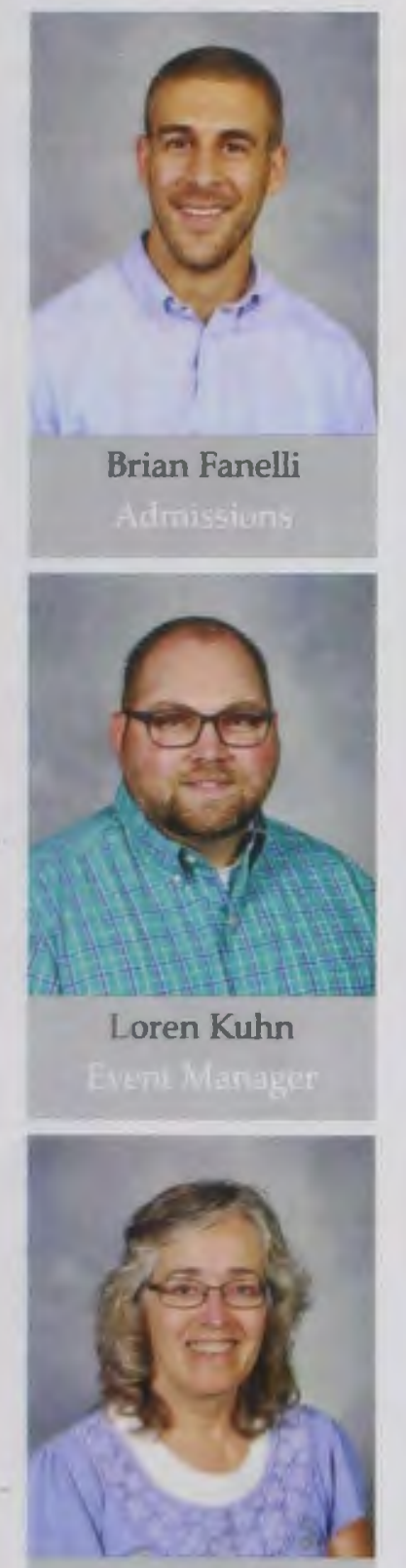

Kay McFarlane

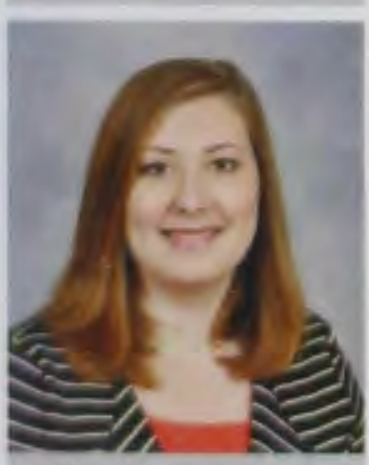

Kirsten Setzkorn

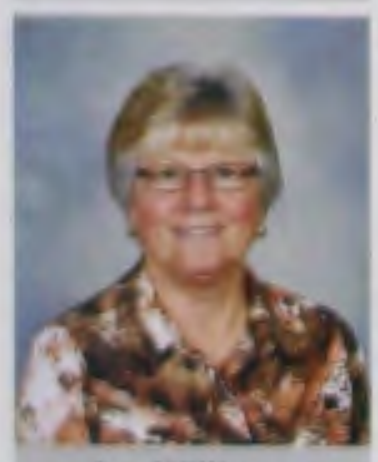

loy Williams

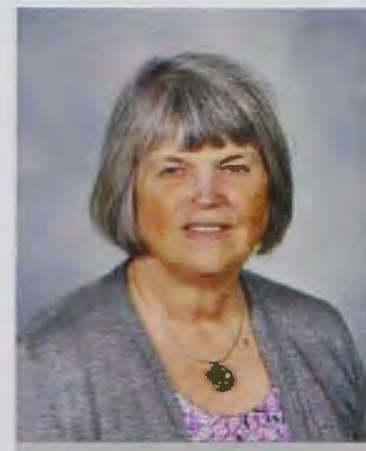

Cindy Gayer

duroation Staif

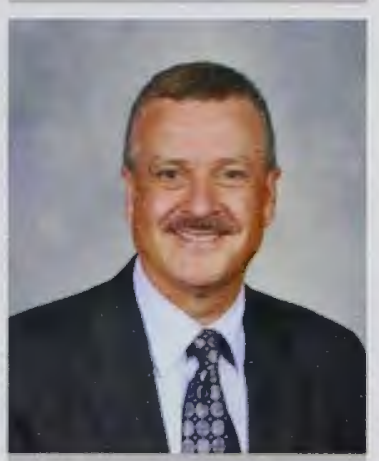

Brian Kuyatt
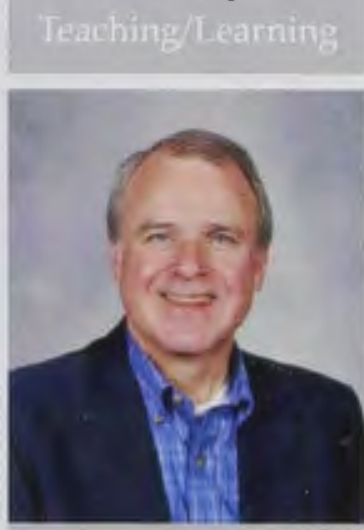

Brian Nester

Chabal wintmen

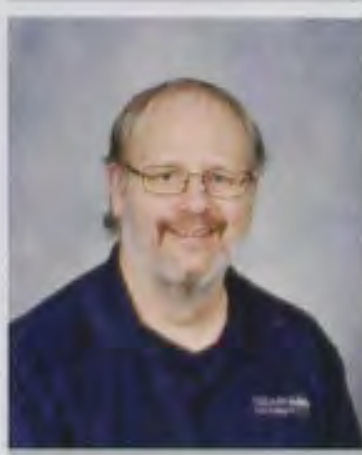

Larry Scott

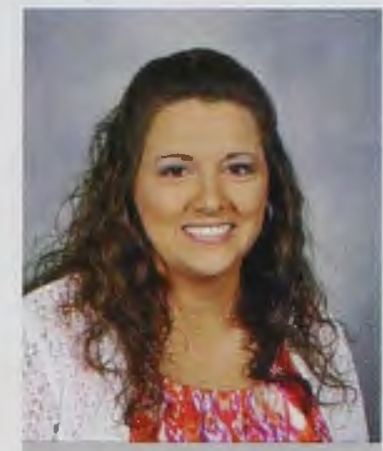

April Hand

Techinical Asst.

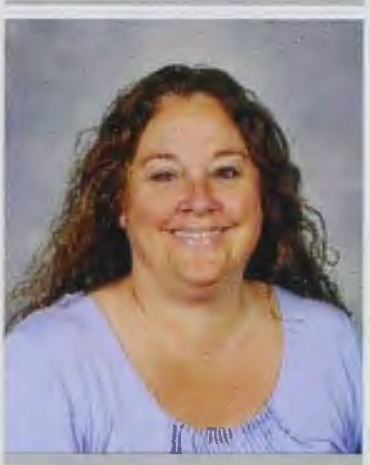

Laura LeMaster

Technical Asst.

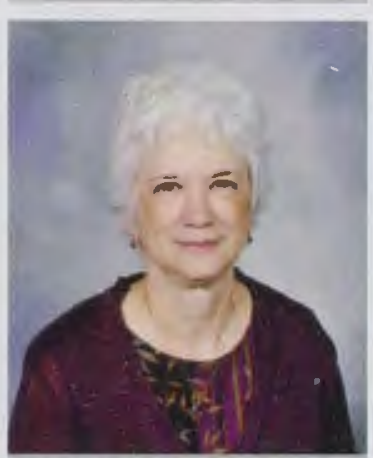

Luann Nicholas

Librain Staff

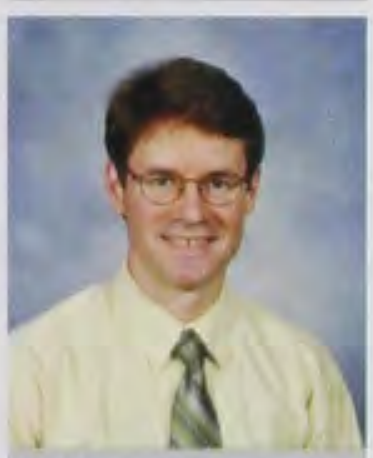

Michael Shepherd

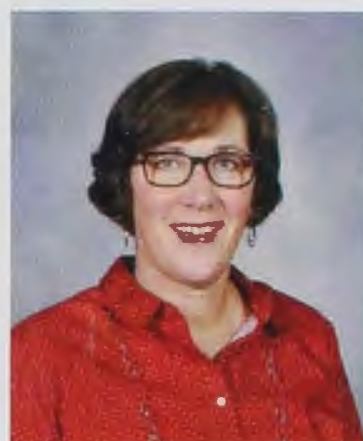

Val Harmon
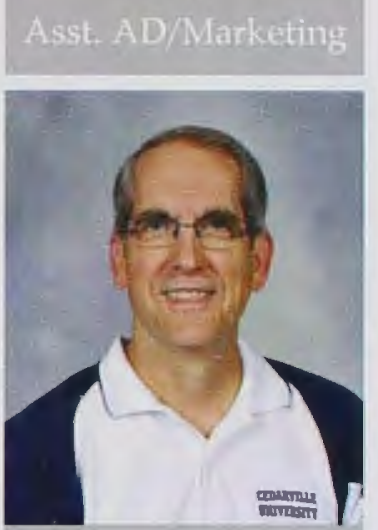

Mark Mathews

Campus Res

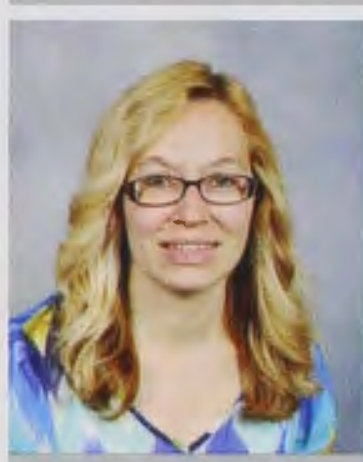

Christina Penrose

Adinn. Asst.

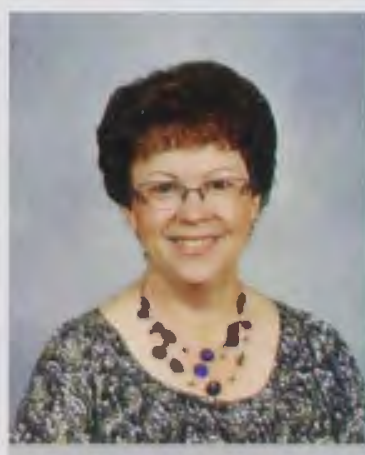

Cheryl Shupe
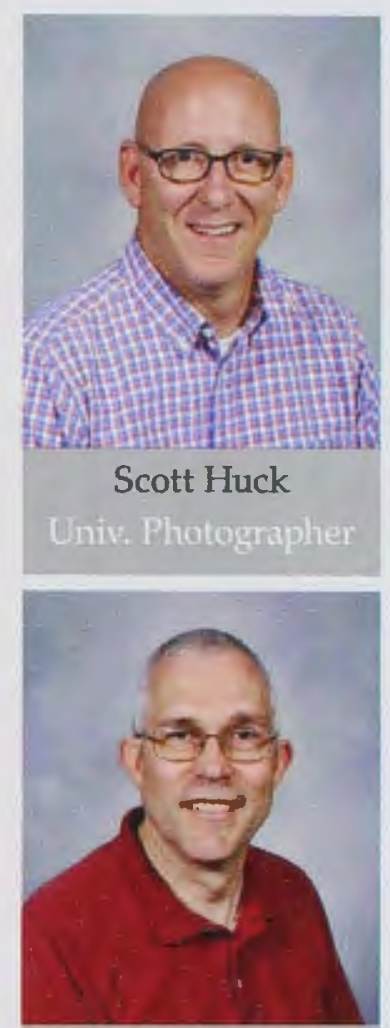

Alan McCain

Information Tech.

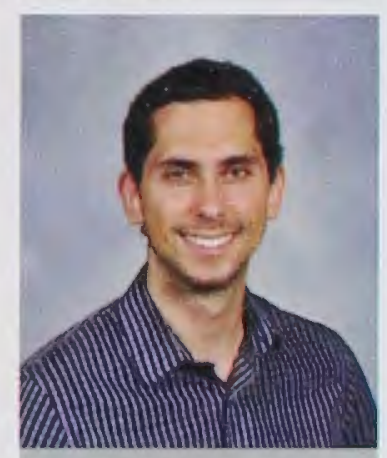

Stephen Port

Admissions

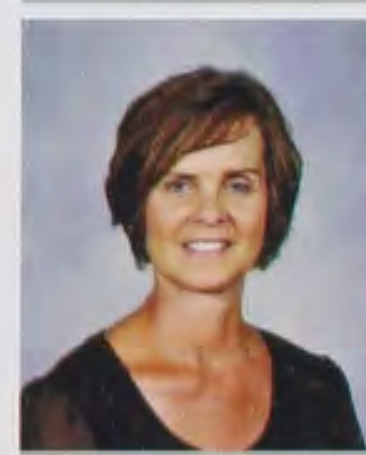

Kimberly Wade

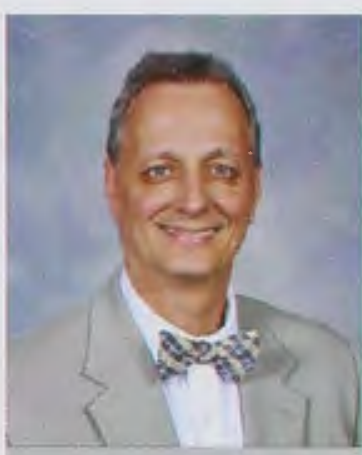

Kim Jenerette

ctrinamotal A A

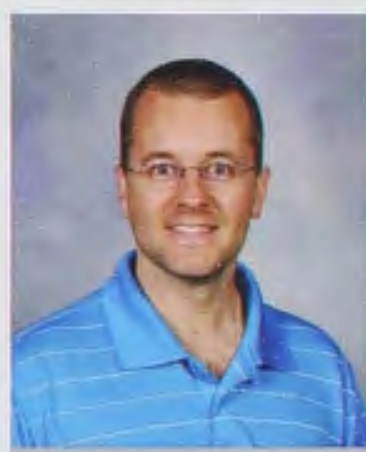

Devon McCarty
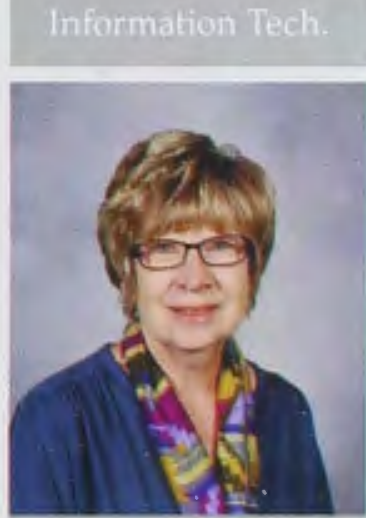

Jackie Pyles

Admn. Asst.

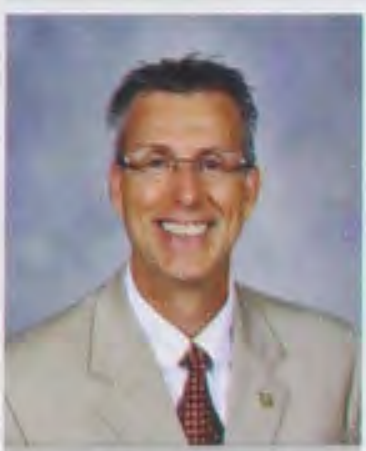

Mark Weinstein 


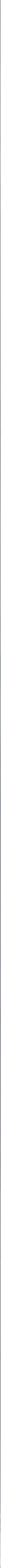




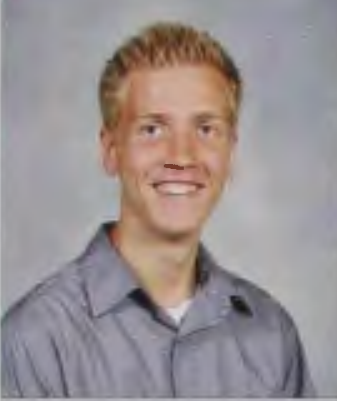

Benjamin Adam

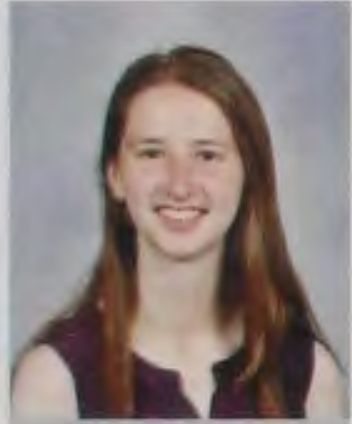

Rebecca Amacher

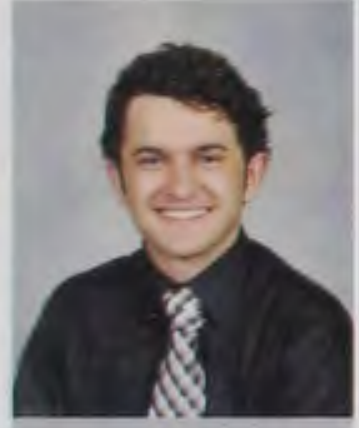

Jesse Arn

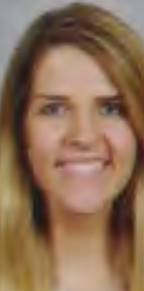

Julie Ballentine

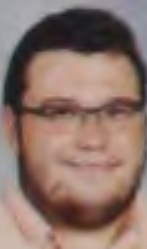

Andrew Beckett

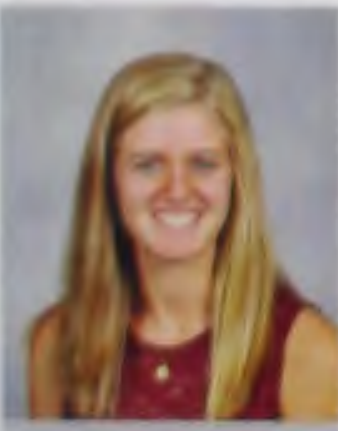

Hannah Boardman
Jacob Adams

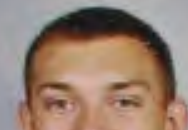

$\Leftrightarrow$ Ais

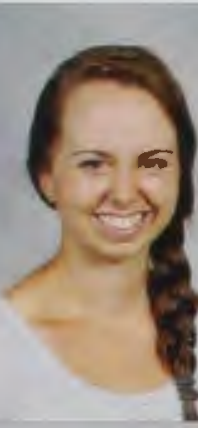

Olivia Ancil

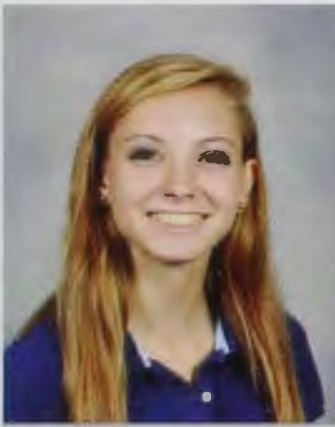

Erin Ashley

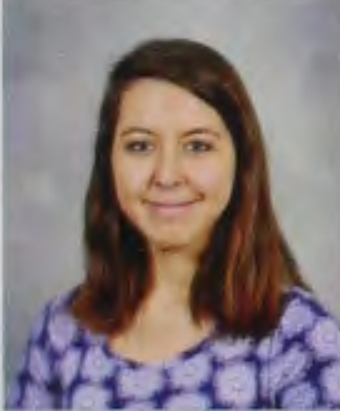
Amanda Barahona Maurer

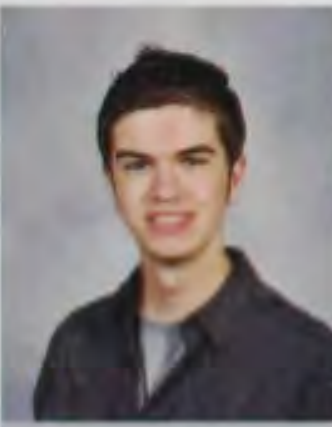

Christopher Bell

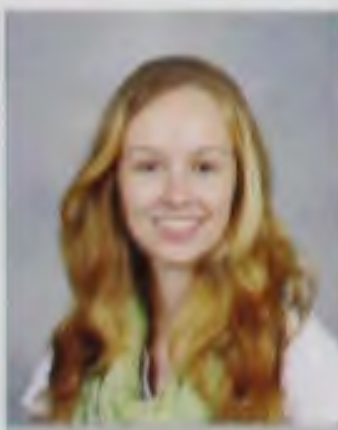

Julia Bonifield

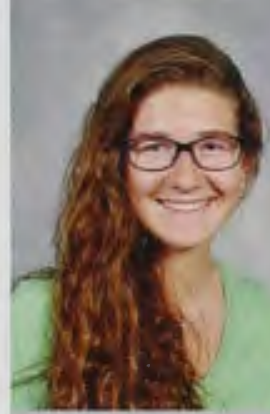

John Barfell
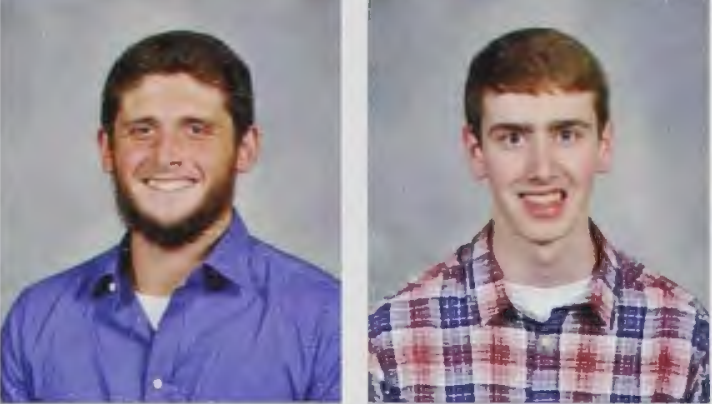

Bryson Adkins

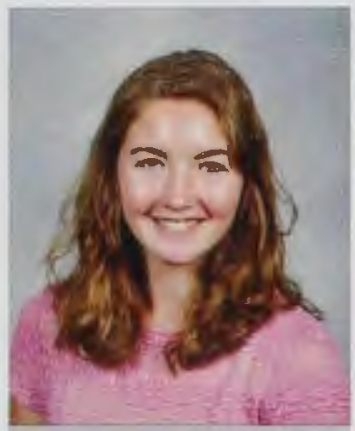

Emily Anderson

Rebekah Athineos
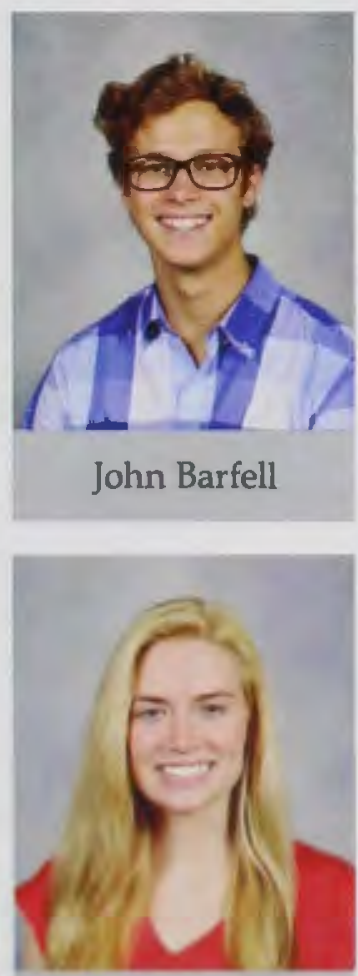

Madison Bender

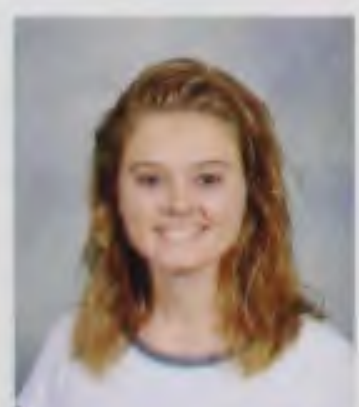

Taylor Bormann
Corey Adkins

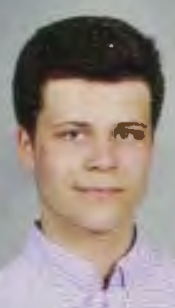

Jared Andre

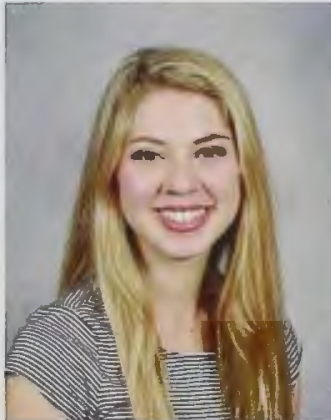

Emma Baccus

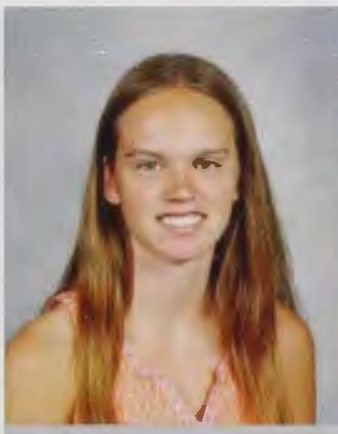

Julia Barker

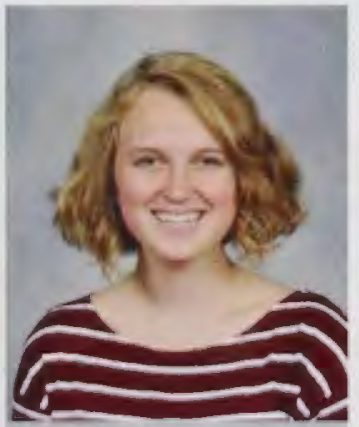

Alyssa Bergthold

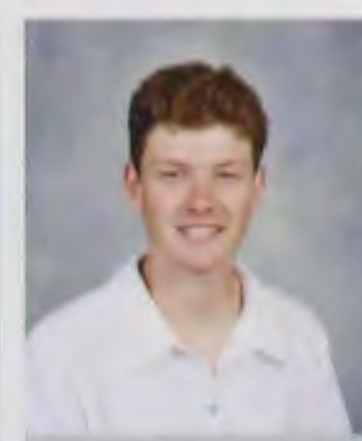

Campbell Bortol
Nathaniel Albrecht

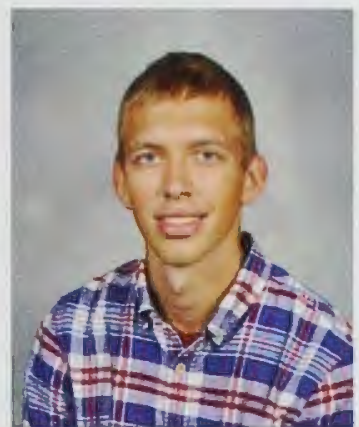

Caden Andrick

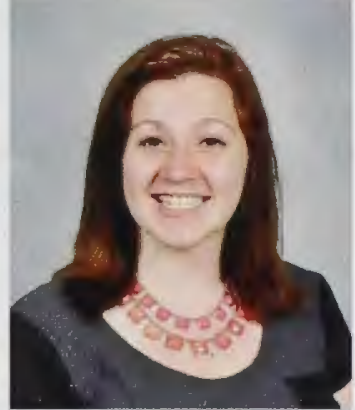

Alexis Baird

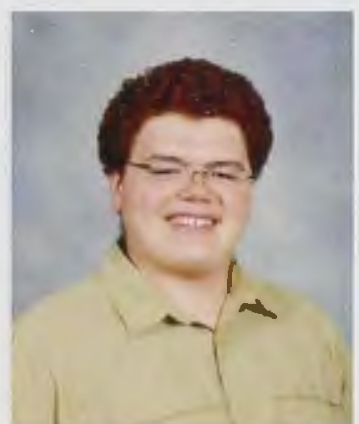

Nathan Barlow

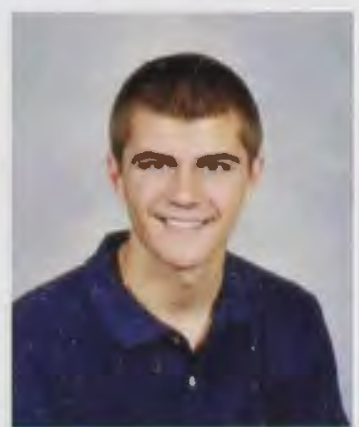

Stephen Berrill

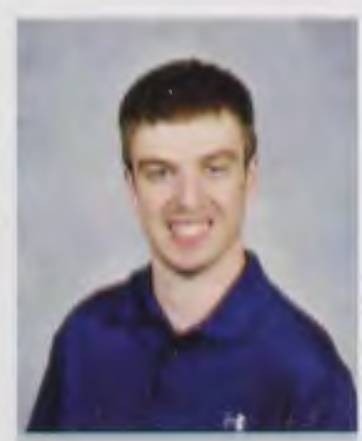

Berjamin Bowden
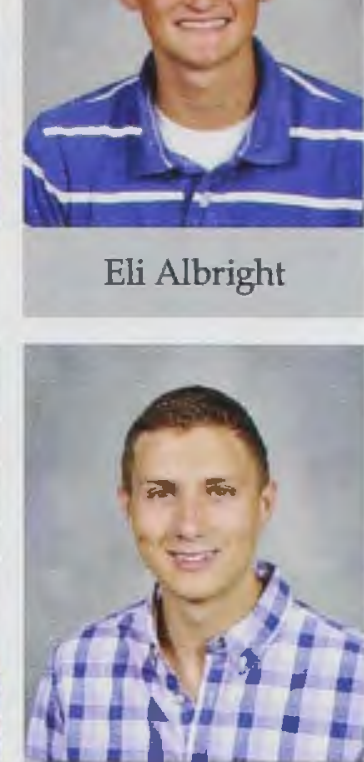

Eli Albright

Alexander Angus

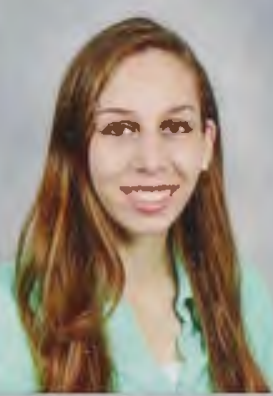

Rachel Balentine

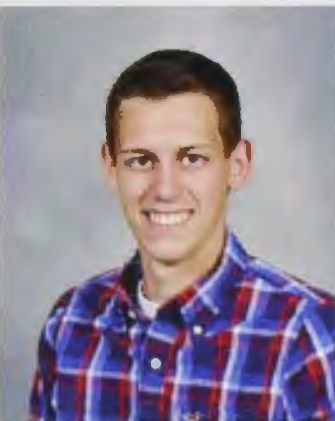

Trey Barnes

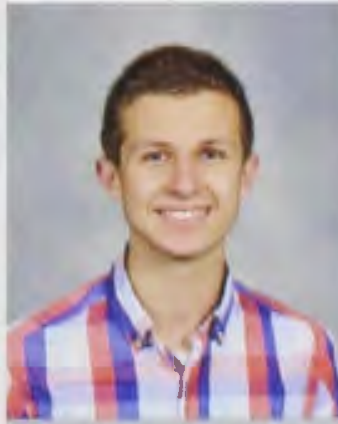

Zachary Bindus

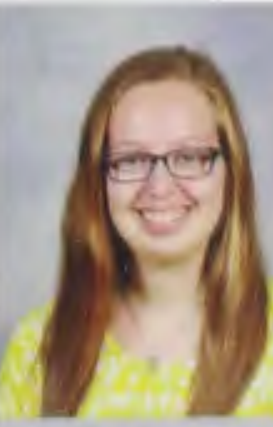

Trane Bowersock

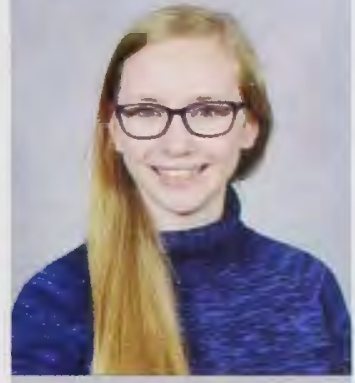

Christine Applegate

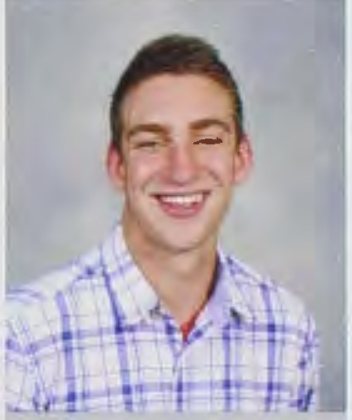

Andrew Ballentine

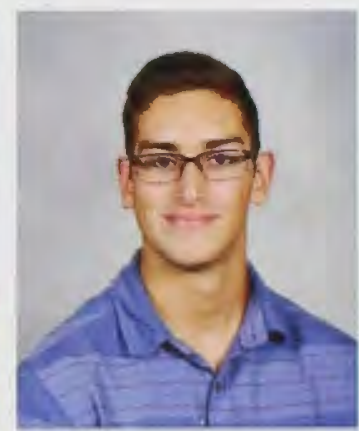

Andre Baumann

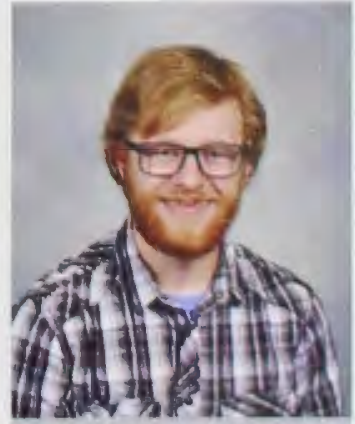

Phillip Birt

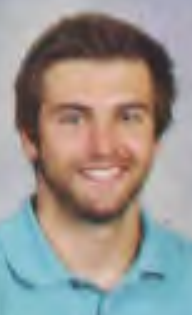




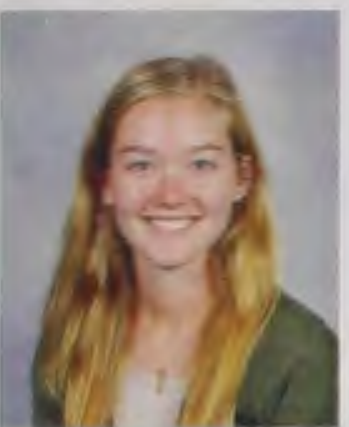

Lindsey Dunn

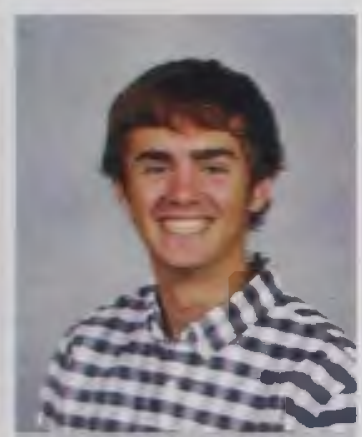

Vance Ehrlich

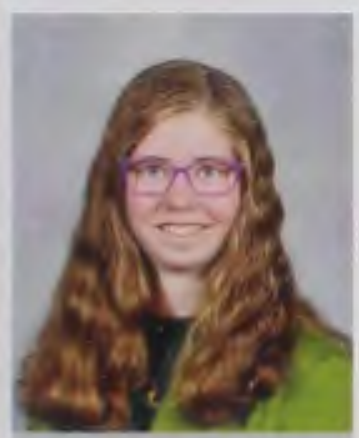

Jessica Evans

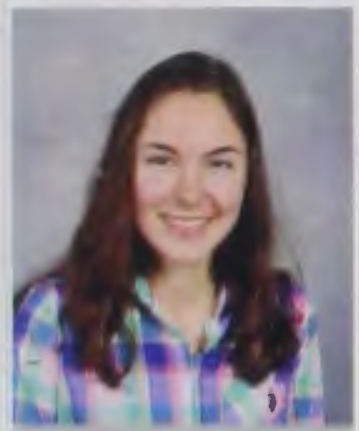

Grace Flint

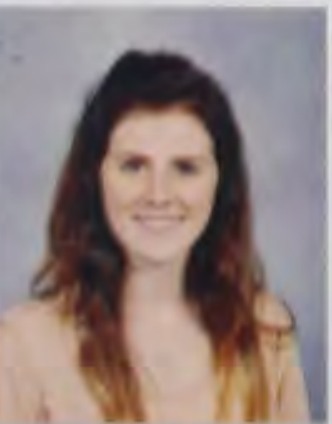

Francesca Garnica

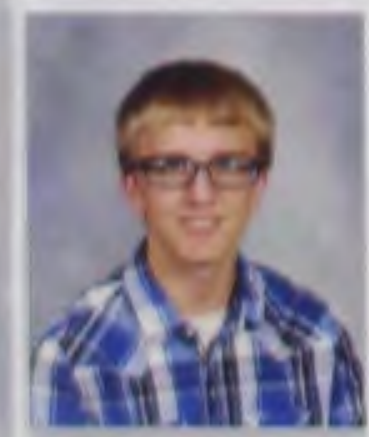

Matthew Glick

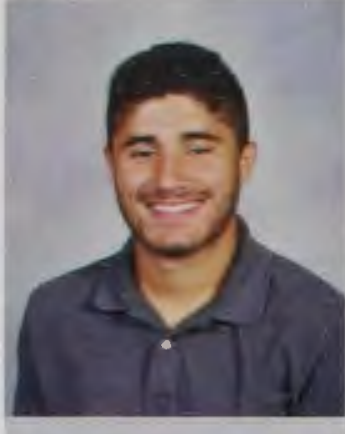

Sebastian Duque

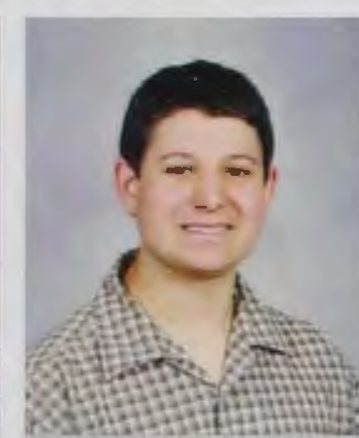

Samuel Ellicott

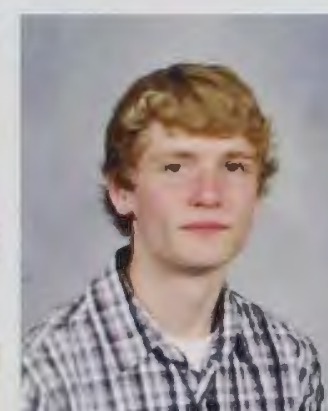

Garrett Fairburn

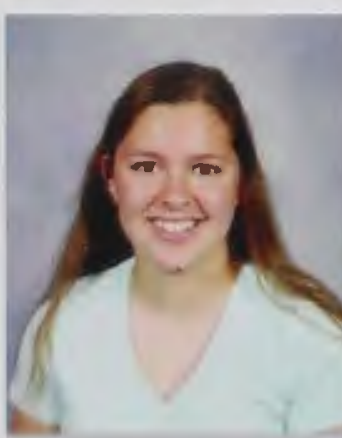

Alexis Ford

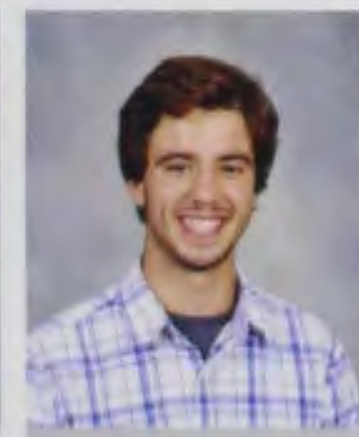

Jonathan Gaunt

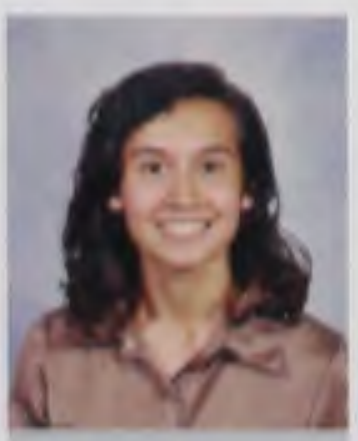

AsiaMay Cood

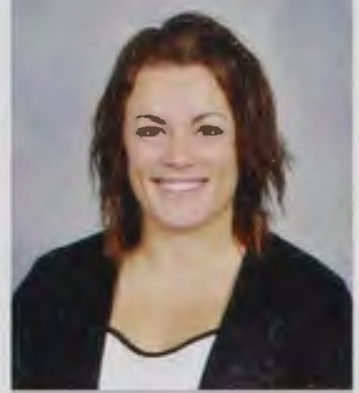

Julie Dyer

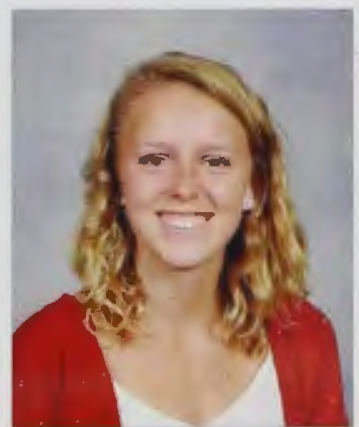

Brianna English

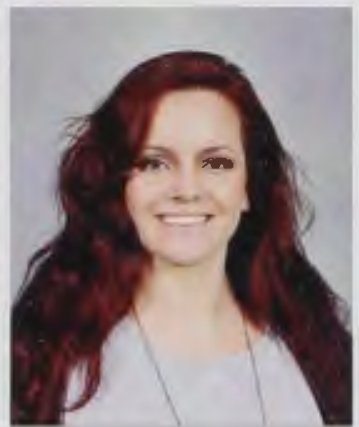

Masie Fedako

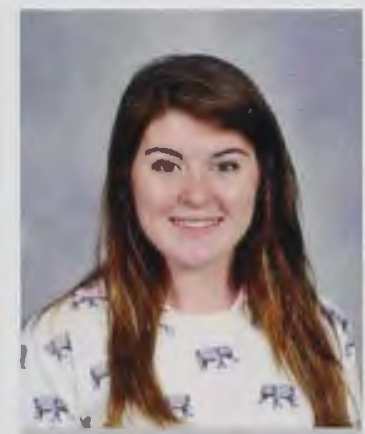

Robinn Fornwalt

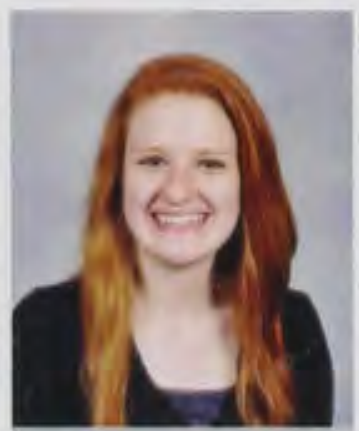

Shannon Gebauer

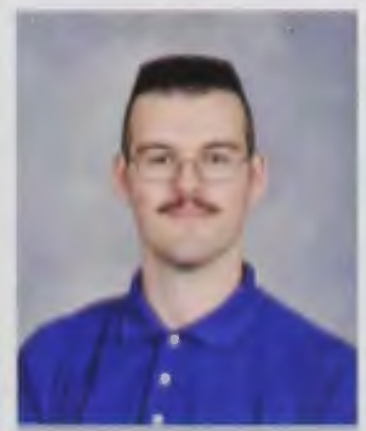

Ryan Gordon

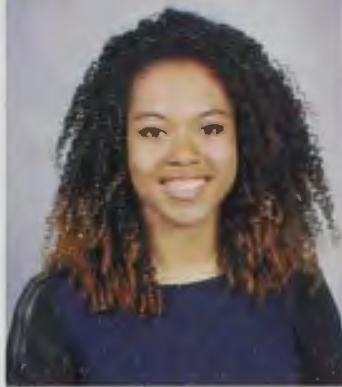

Miranda Dyson

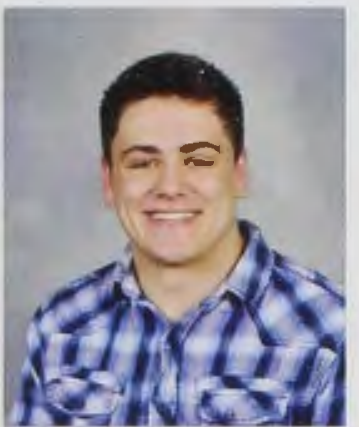

Dean English

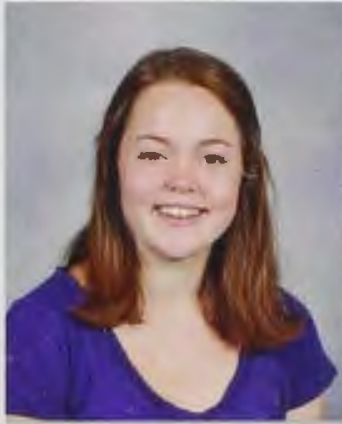

Madeline Feiser

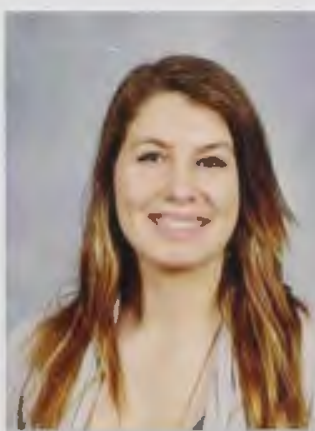

Hannah Fritz

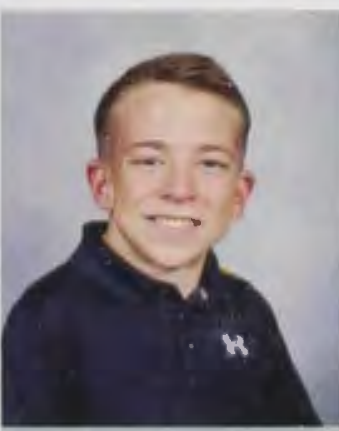

Brennon Gee

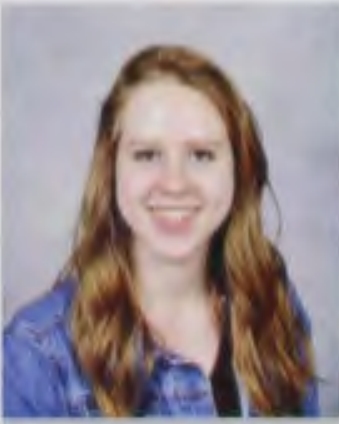

Abigail Gray

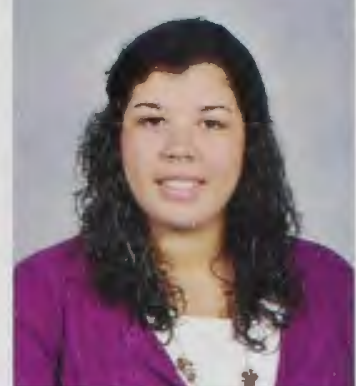

Emily Edwards

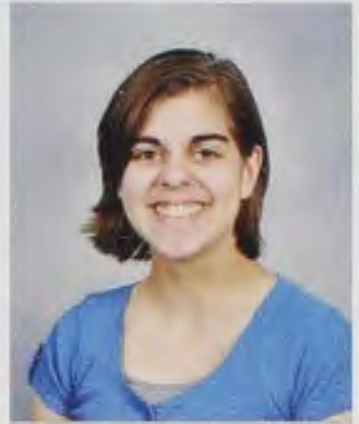

Emily Entner

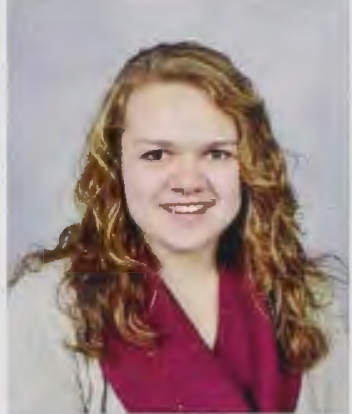

Katelyn Fisher

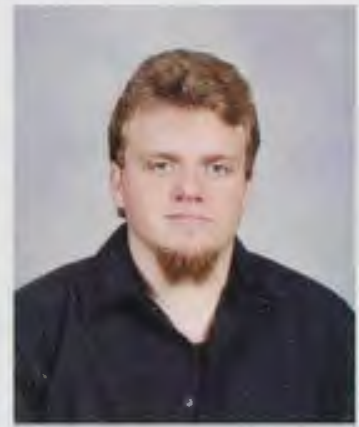

James Frizzell

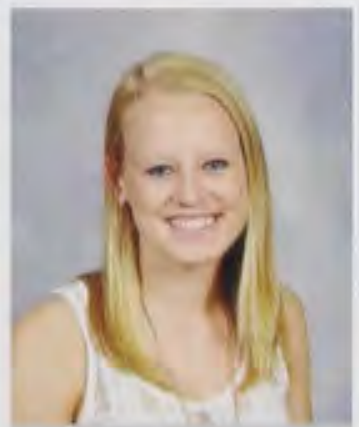

Marisa George

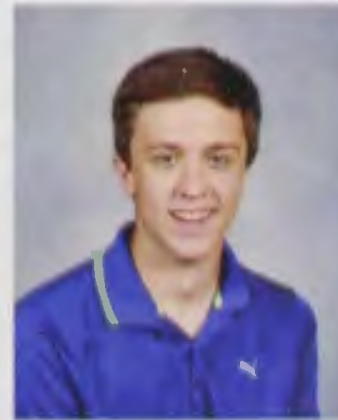

[David Green

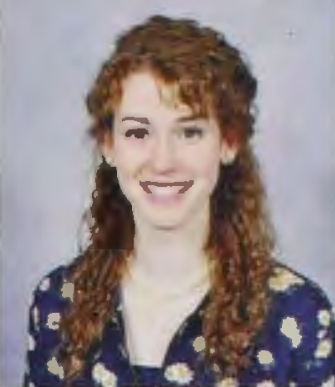

Susanna Edwards

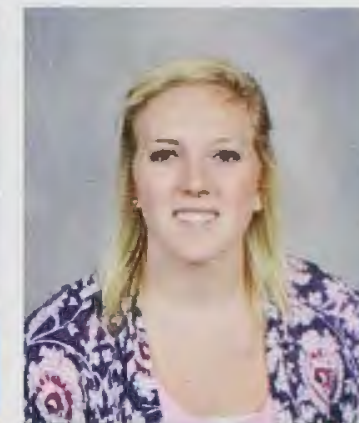

Courtney Eppard

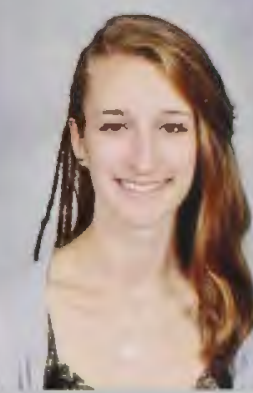

Rachel Fletcher

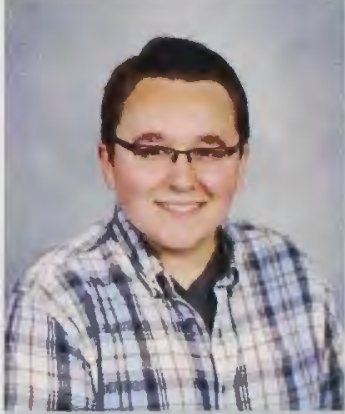

Aaron Gagne

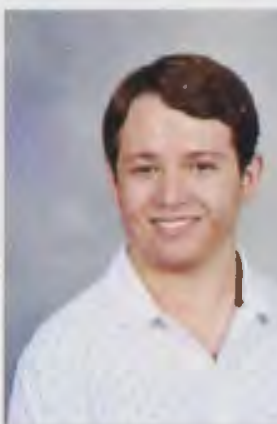

Nickolas Gerwig

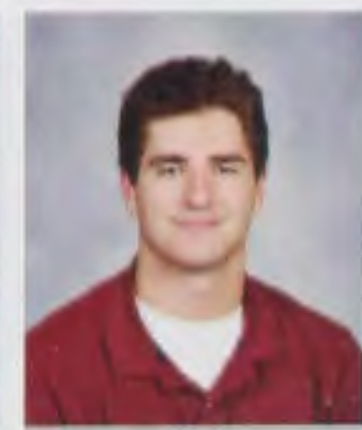

Tamner Green

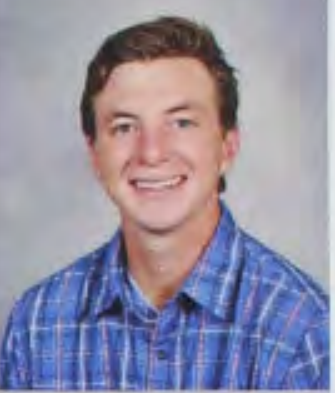

Carter Ehms

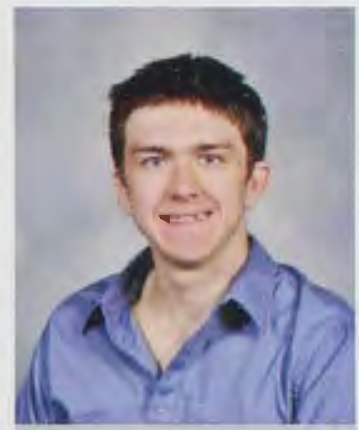

Robert Eppley

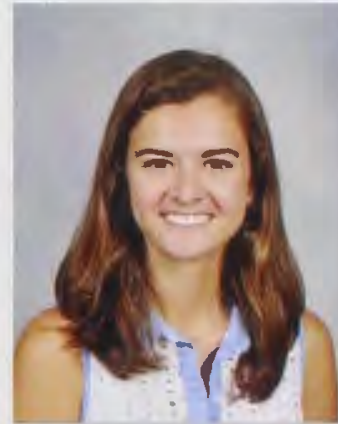

Andreya Flinn

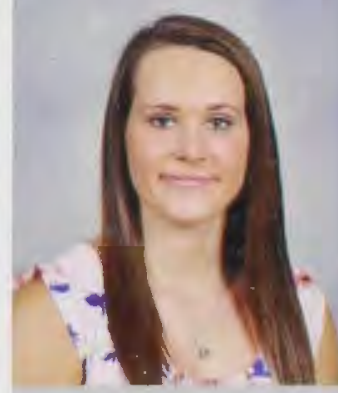

Ashley Gamber

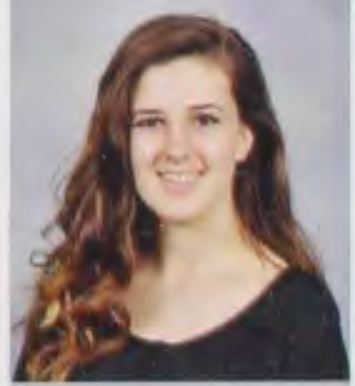

Julia Glaccum

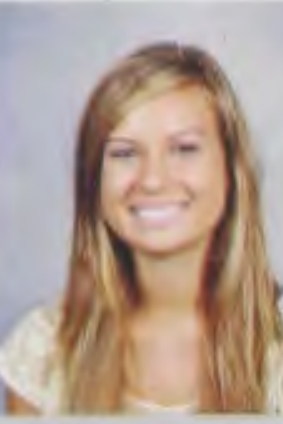

Inna Griffith 


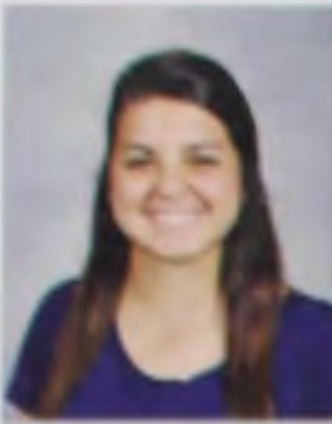

Hope Griffith

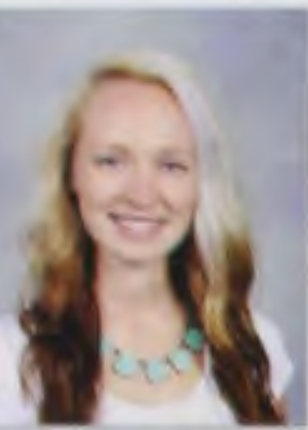

Danielle Haeffner

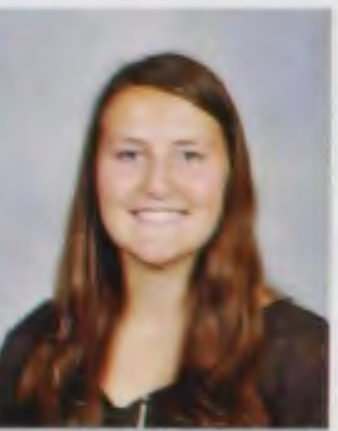

Rebekah Haught

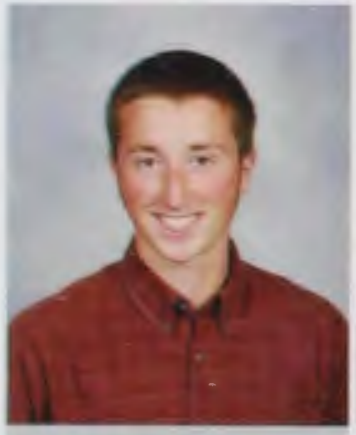

Nathan Herr

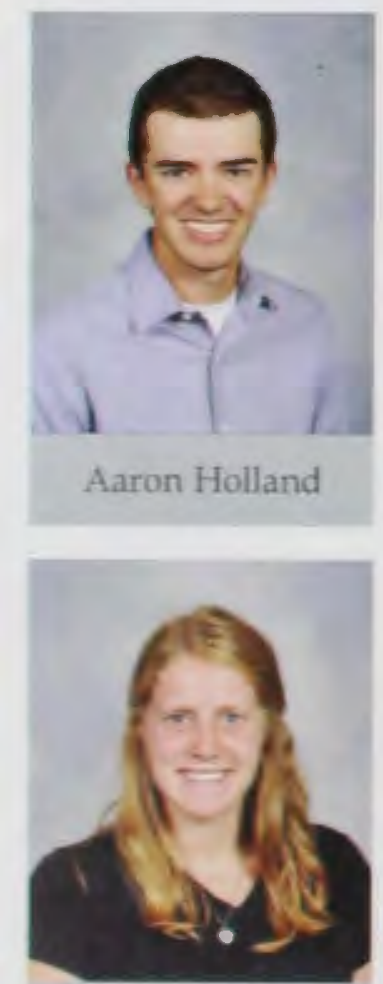

Claire Hoyt

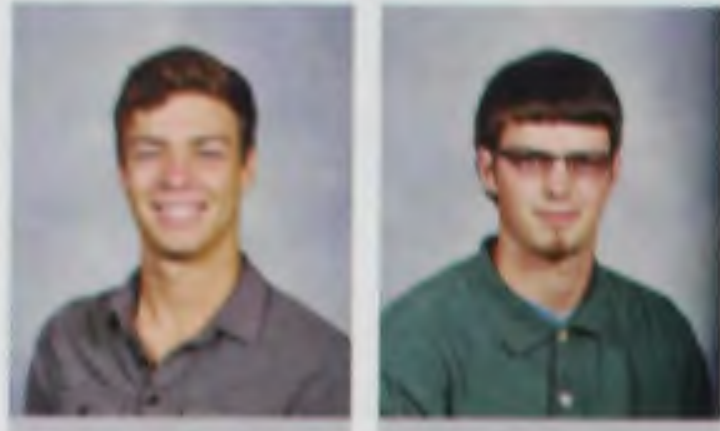

Zachary Ciritfith

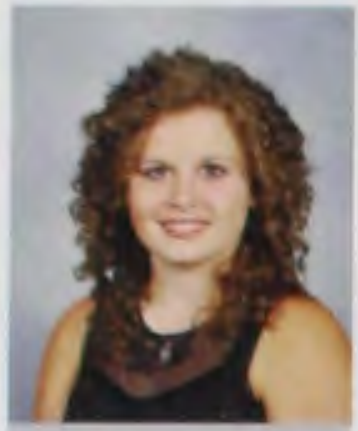

Stephanie Hafler

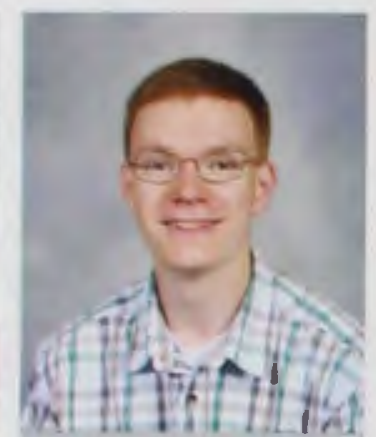

Michael Hayes

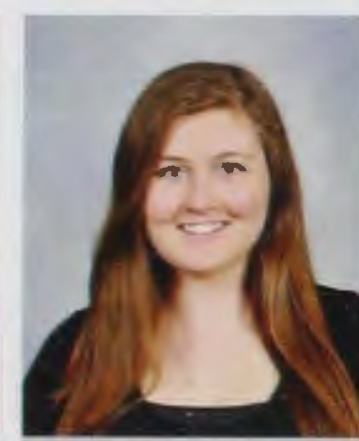

Samantha Higgins

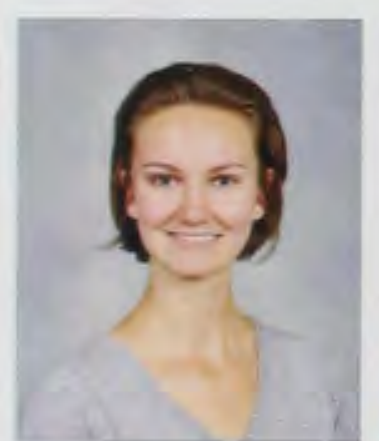

Michaela Holloway

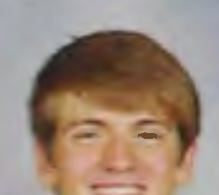

Logan Huck

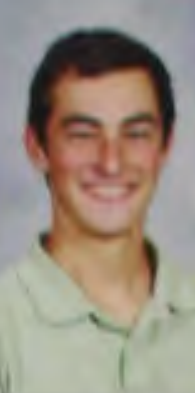

Daniel Grimes

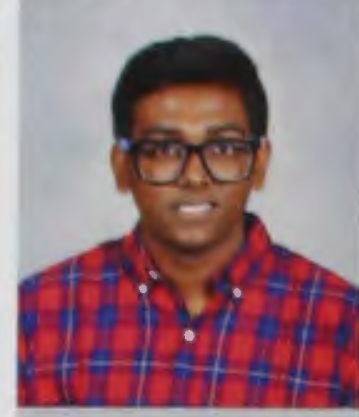

Pius Halder

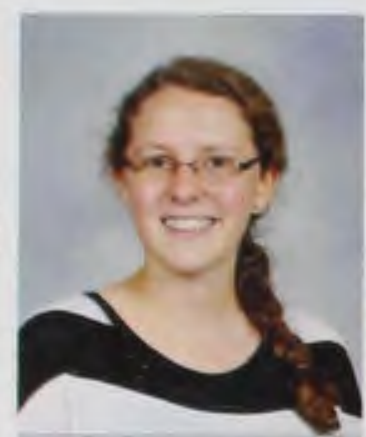

Elena Hein

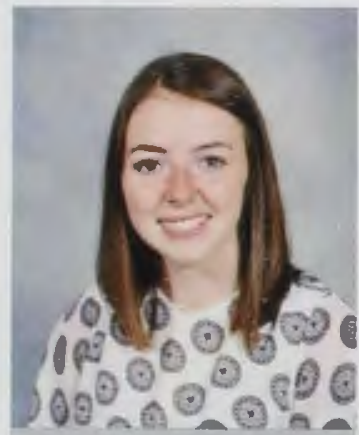

Madison Hille

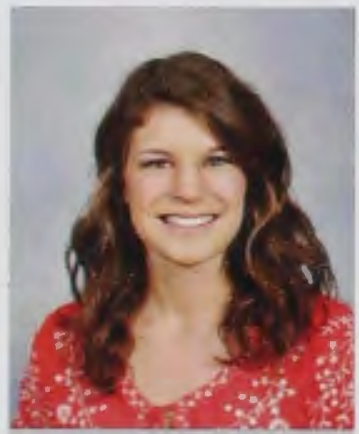

Victoria Holmes

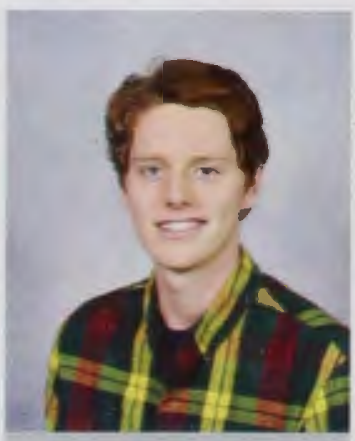

Luke Ingles

Kyle Hooper

Laura Isaacs

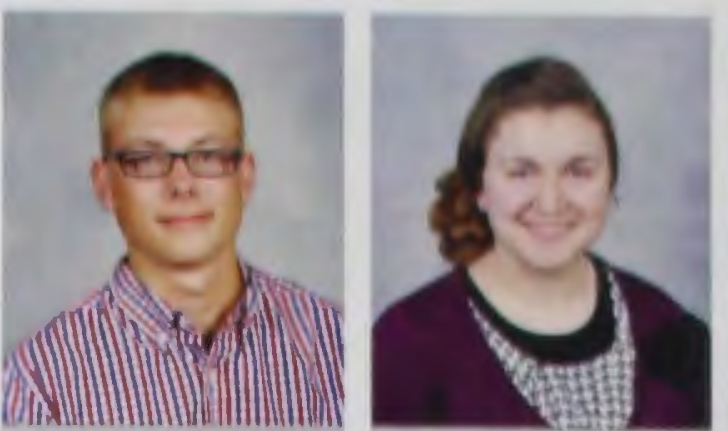

Scoll Grimes

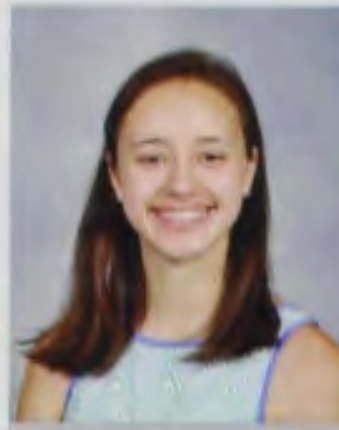

Autumn Hankins

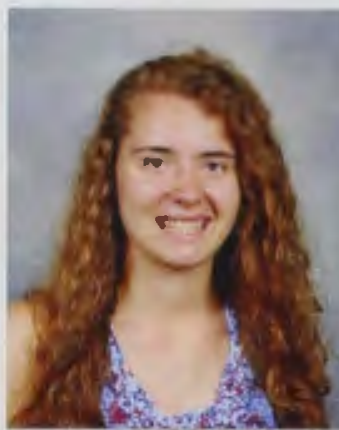

Samantha Heinrich

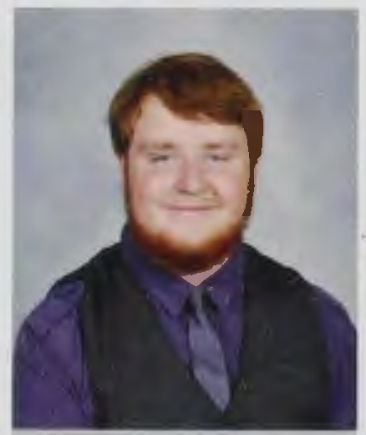

Nathan Hiser
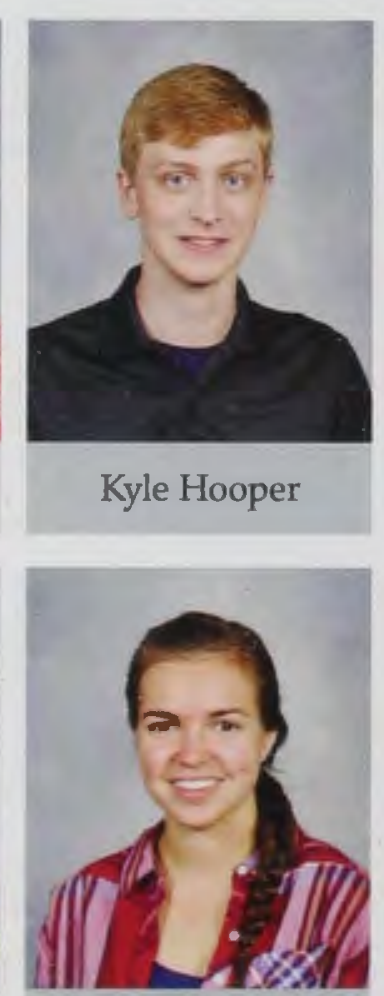

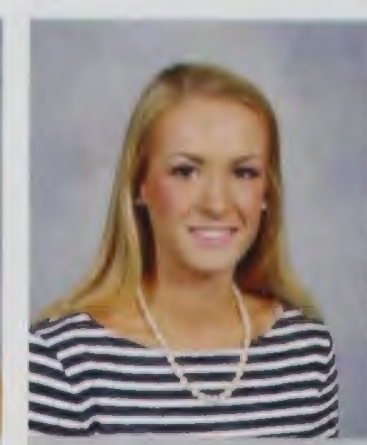

Abigail Hannay

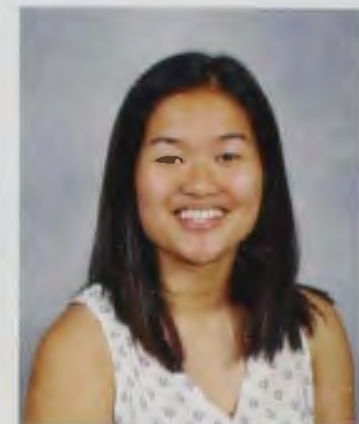

Lily Hellwig

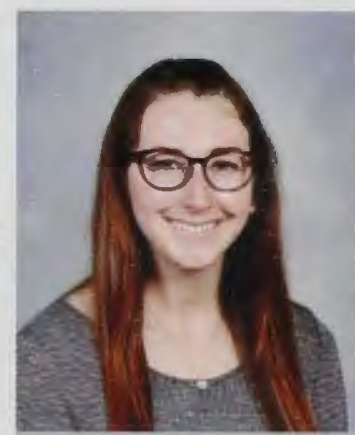

Hannah Holbrook

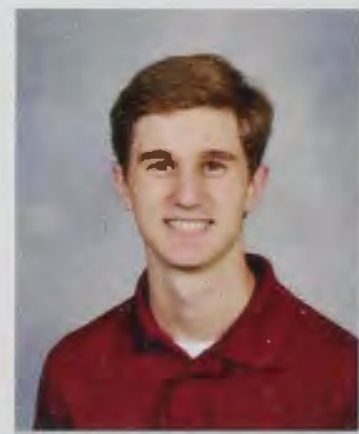

Caleb Hoover

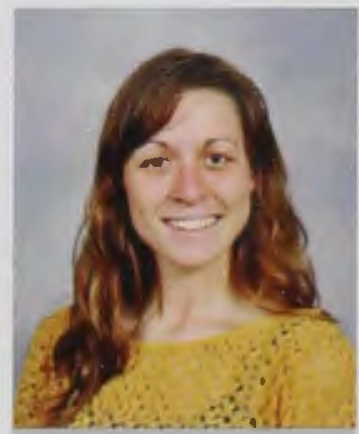

Kourtnie-Sue Ison
Samantha Ciross

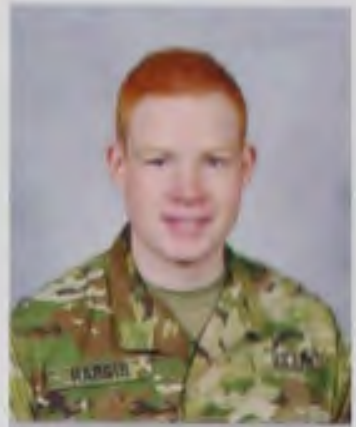

Jordan Hargis

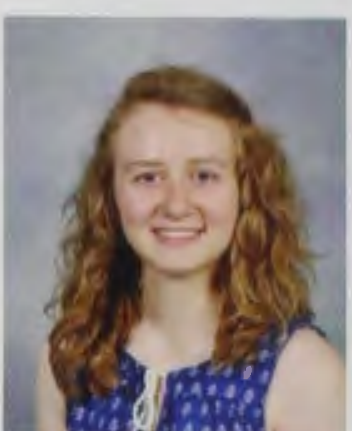

Clara Hendrickson

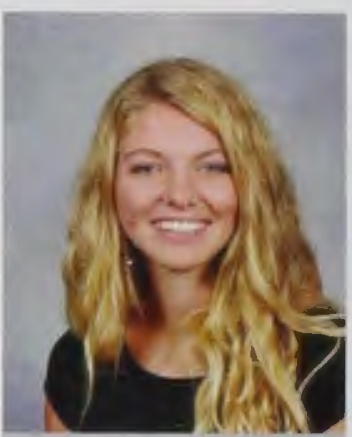

Miranda Holbrook

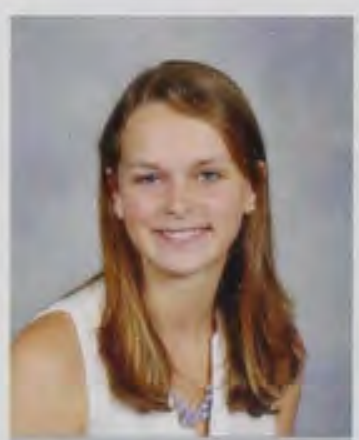

Amanda Horne

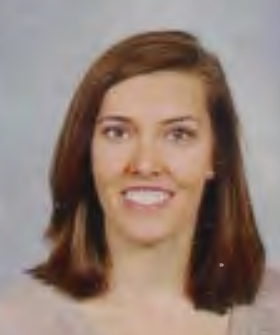

Christiana Jackson

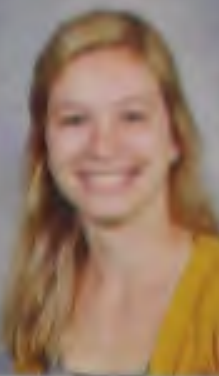

Meagan Cirotte

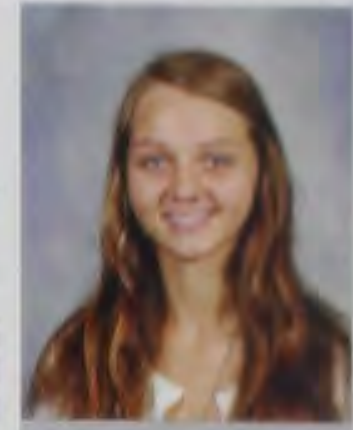

Elizabeth Harkness

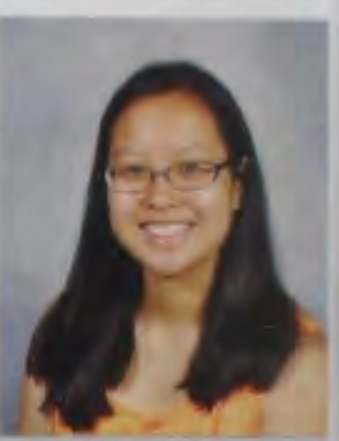

Stephanie Hermann

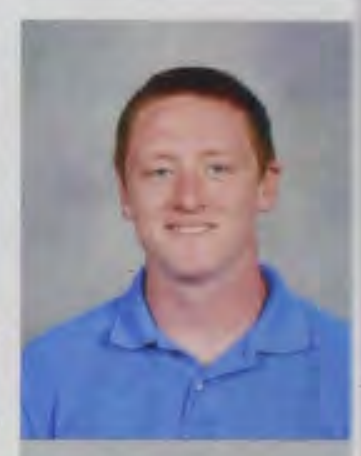

Cameron Holcomb

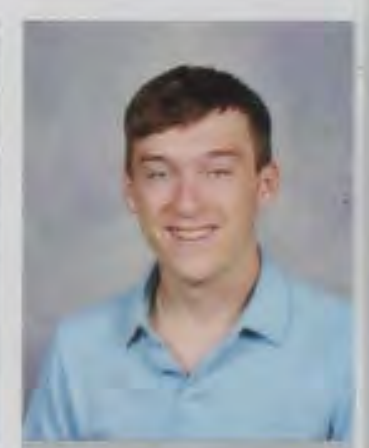

Alexander Hotz

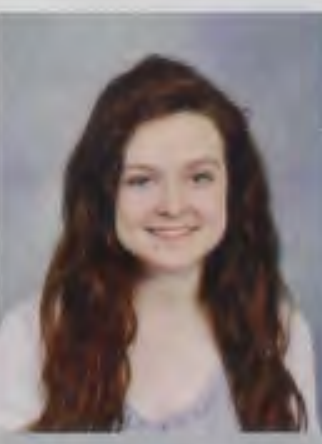

Kristen Jarboe 


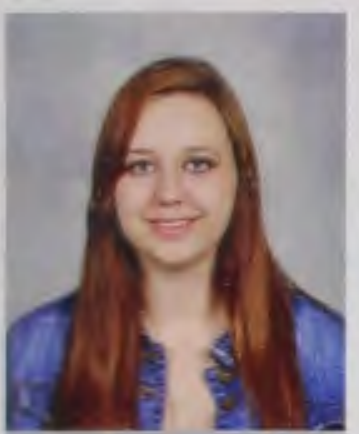

Mikaela Johnson

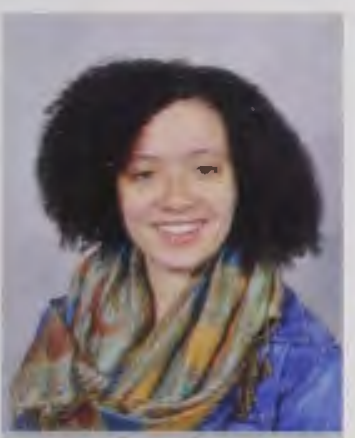

Theresa Jones

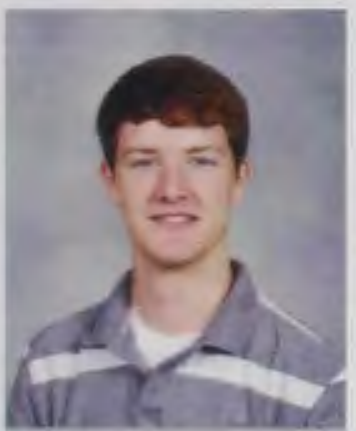

Devan Kienitz

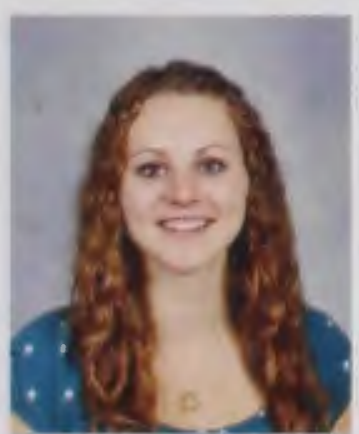

Hannah Klabunde

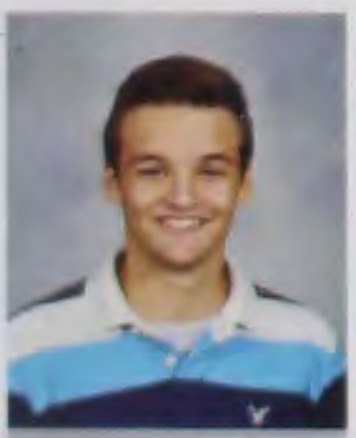

Robert Krupnek.

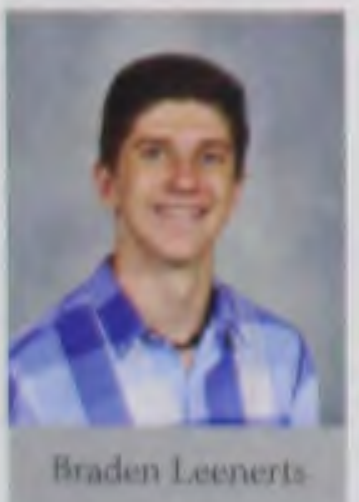

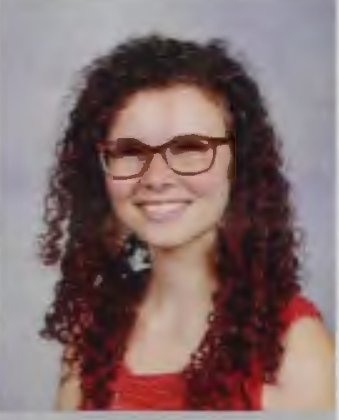

Quintanya Johnson

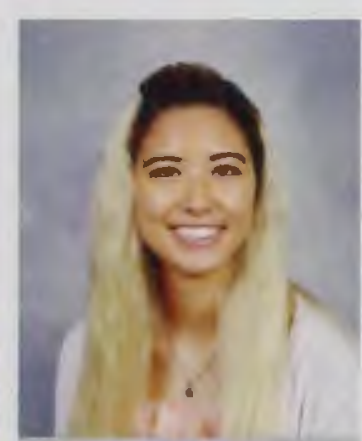

Ayriana Kasper

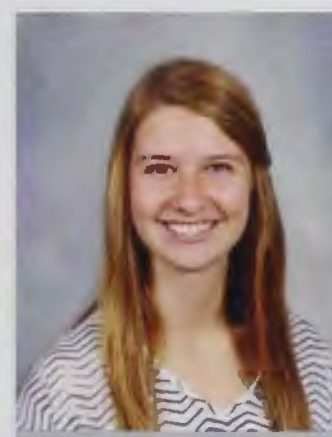

Megan Kimball

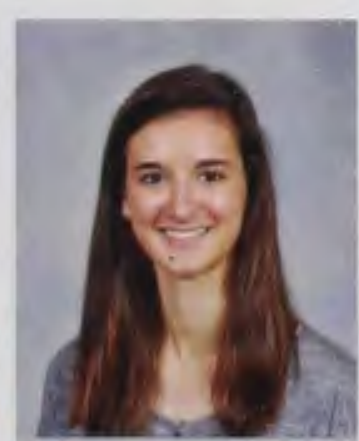

Emily Klein

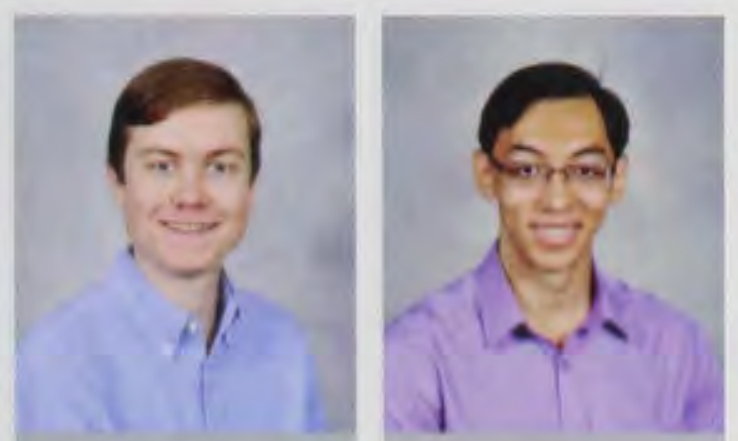

John Kuhn

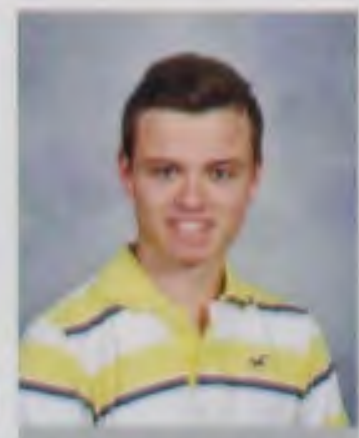

Christopher Leick

Samuel Johnson

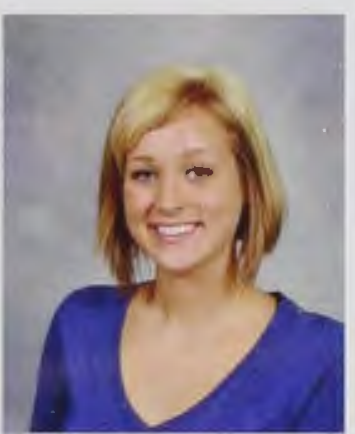

Bethany Kauppila

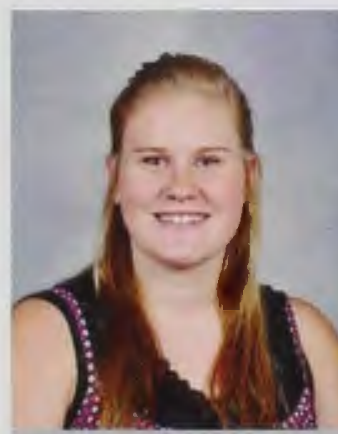

Katelyn Kime

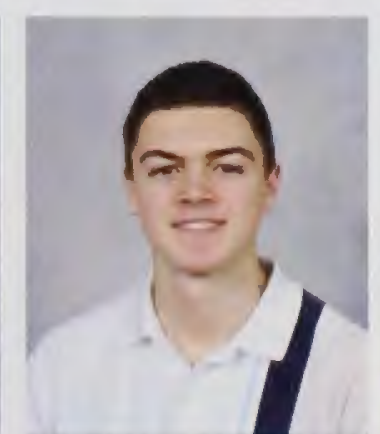

Jared Klimek

Mark LaBarr

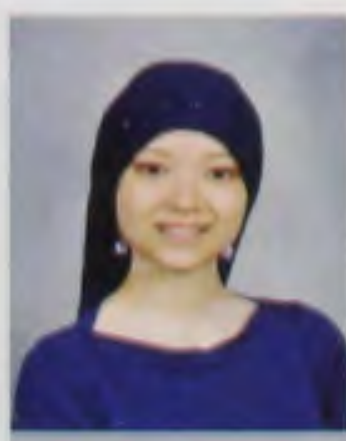

Ailin Leong

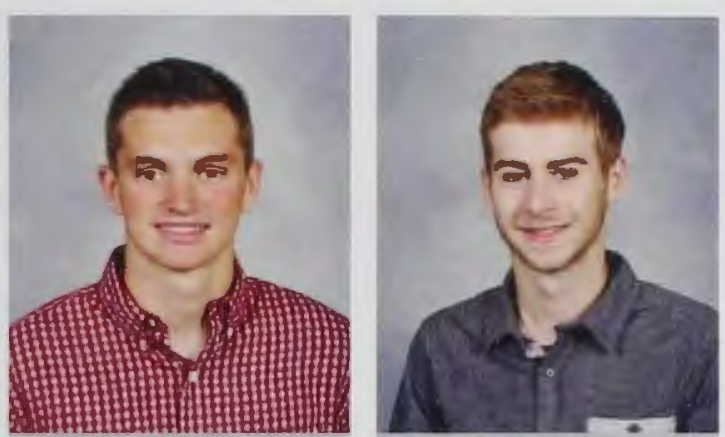

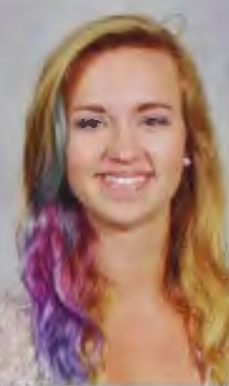

Emily Jones

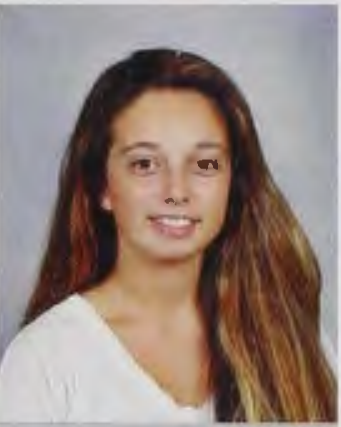

Kirstyn Kelley

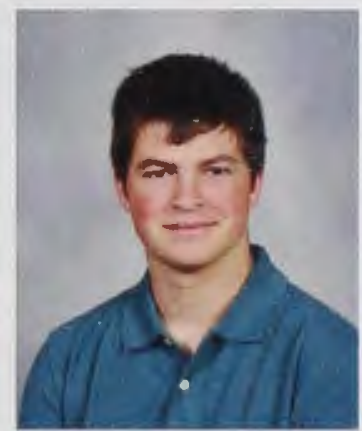

John Kindig

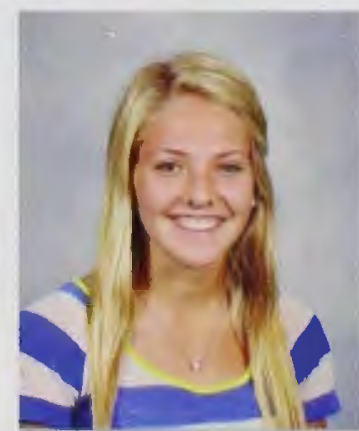

Jenah Korthuis

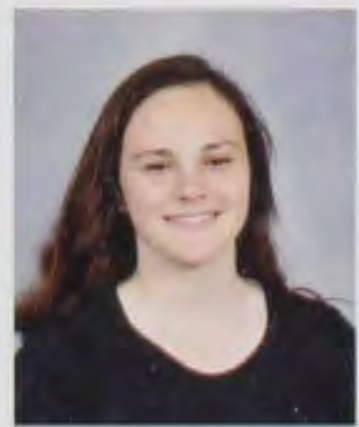

Jaylyn LaFlamme
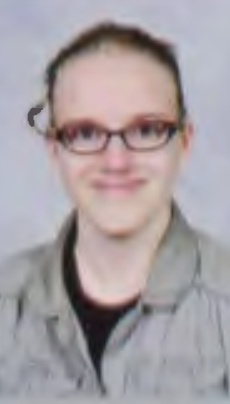

Hen Lewis

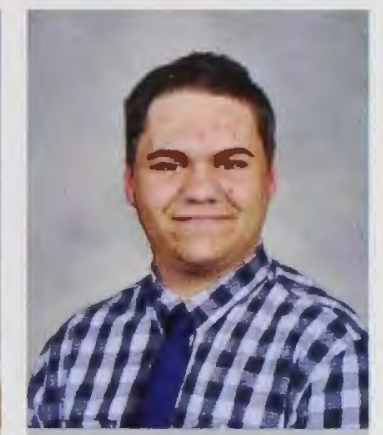

James Kelly

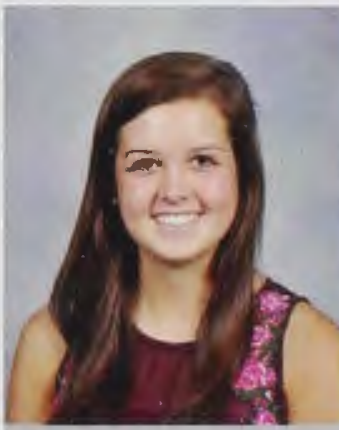

Jenna King

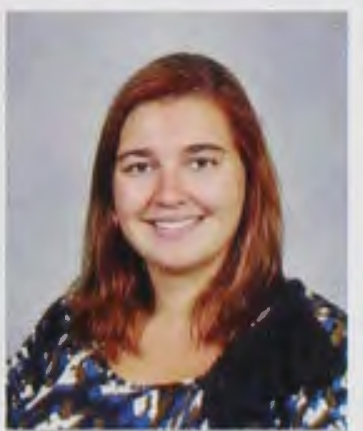

Hannah Kosmicki

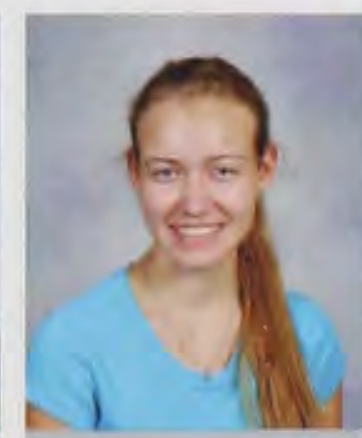

Kristina Lail
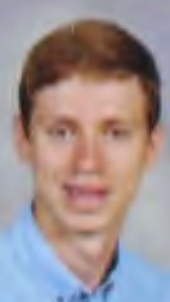

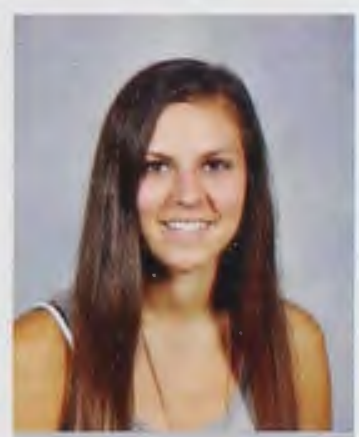

Jordan Kratche

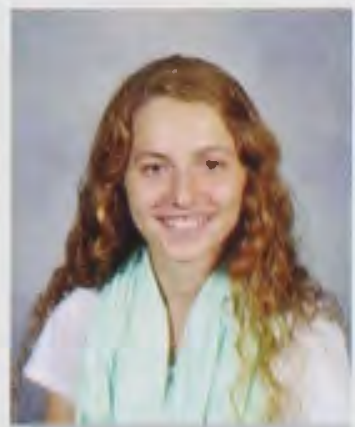

Chloe Lance

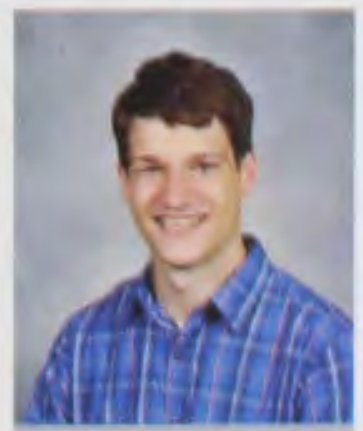

Nathan L inville

Matthew Longest
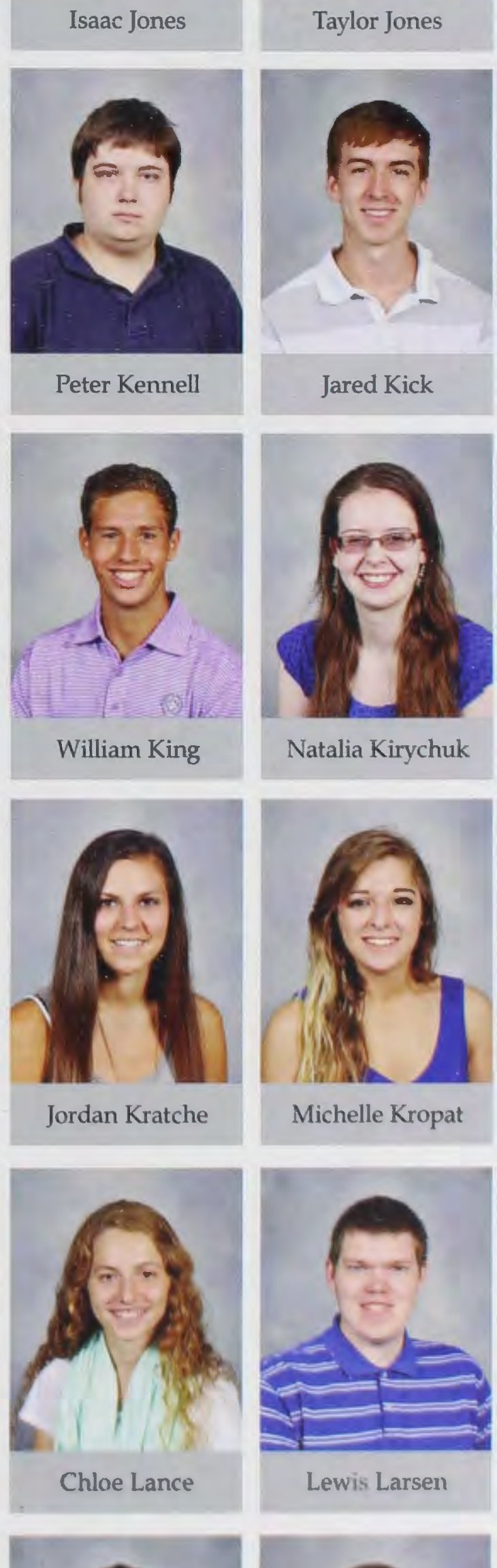

Natalia Kirychuk

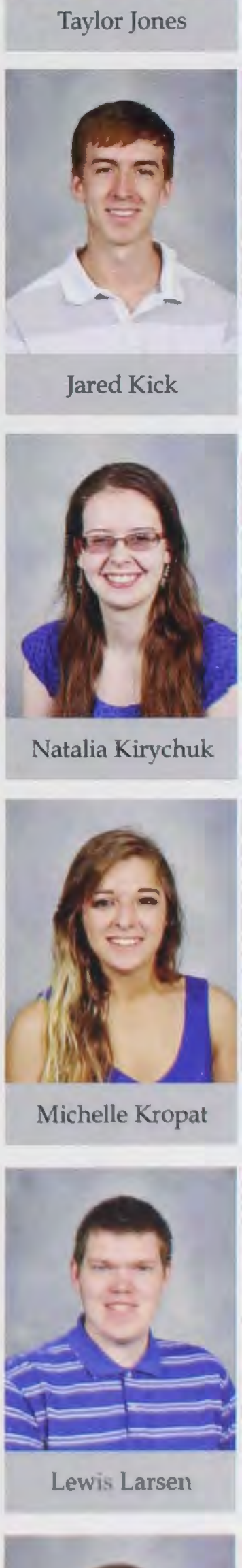

Michelle Kropat

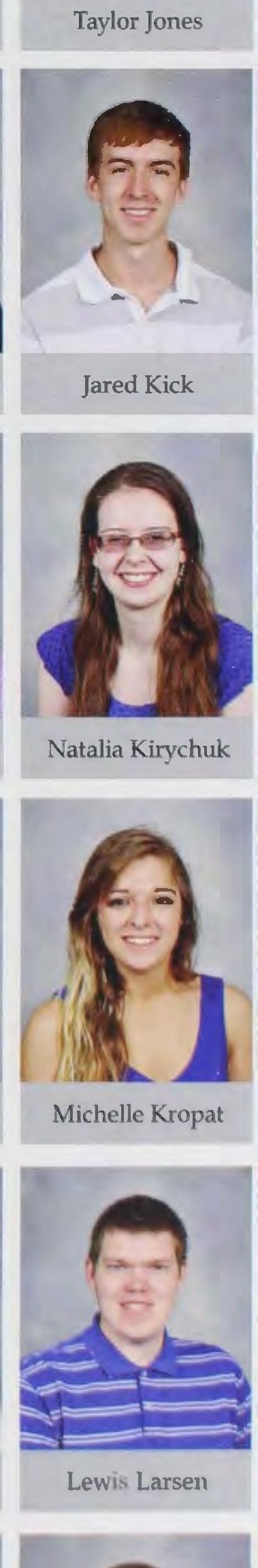

Lewis Larsen

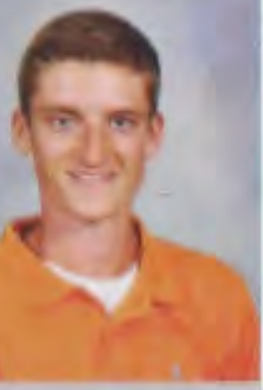

Addison Love 


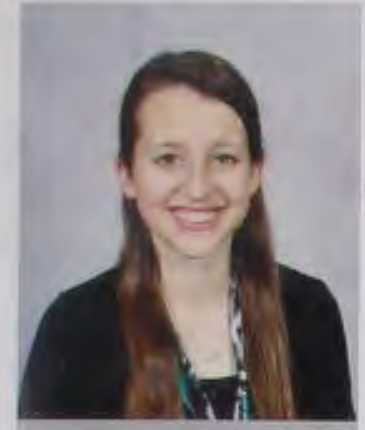

Kristy Parks

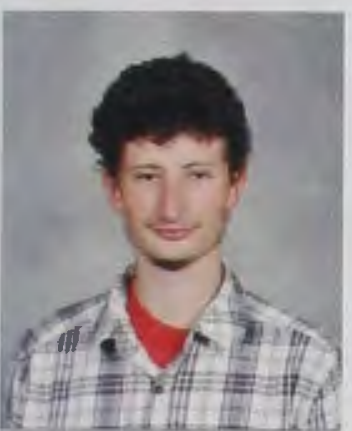

Tyler Penko

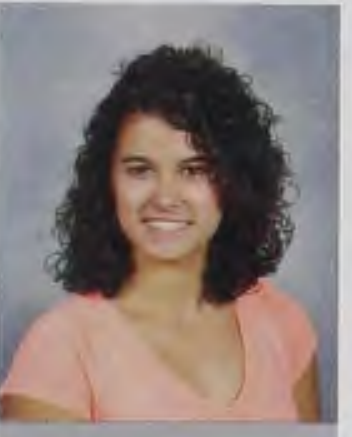

Andriana Polsdorfer

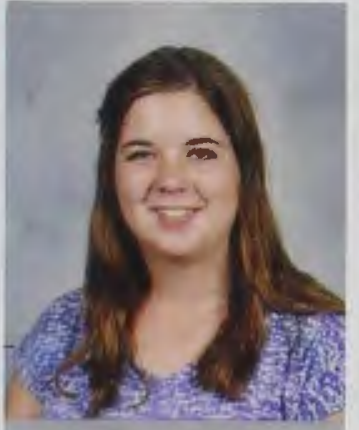

Kaelyn Ratcliffe

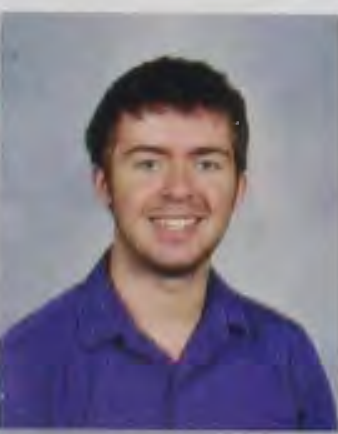

Daniel Ringenoldus

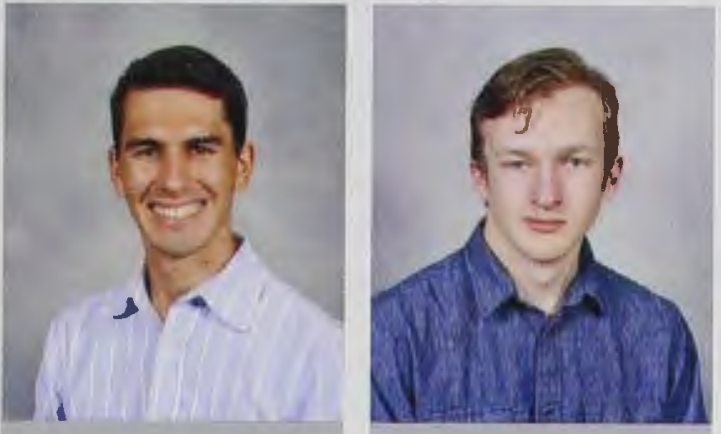

Daniel Parschauer
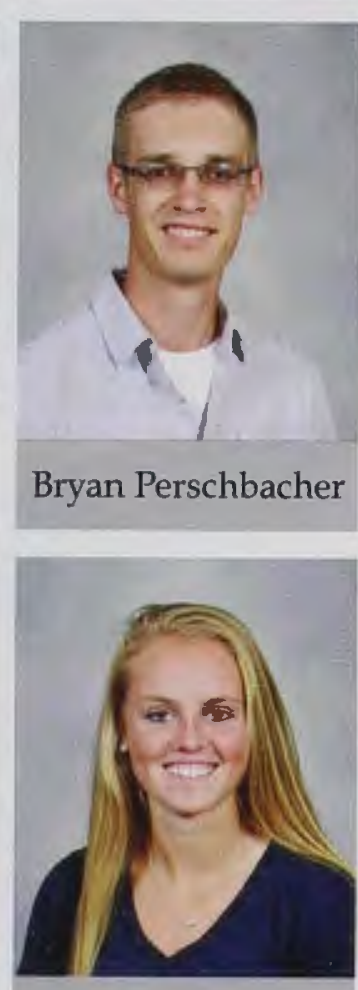

Kelly Poole

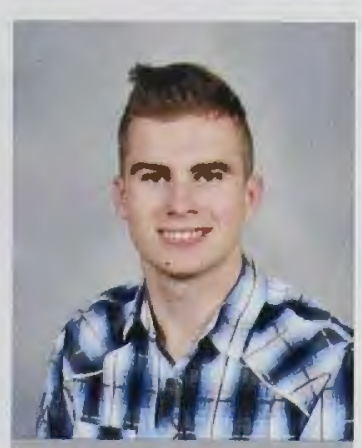

Joshua Ratliff

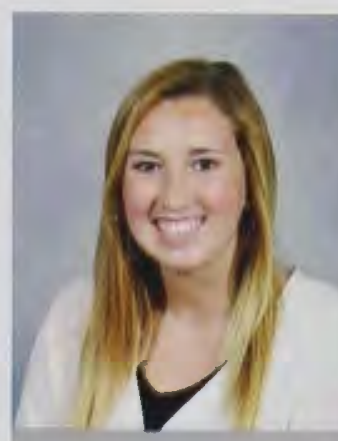

Samantha Rogers

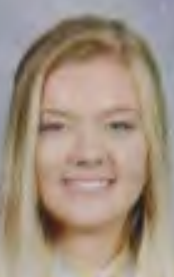

Abigail Russell

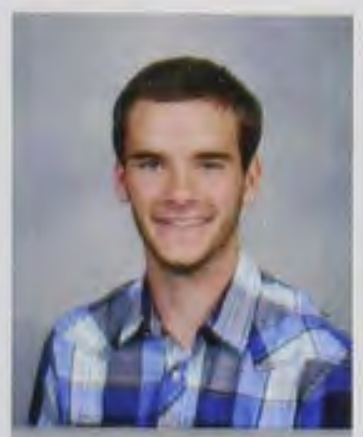

James Ryan

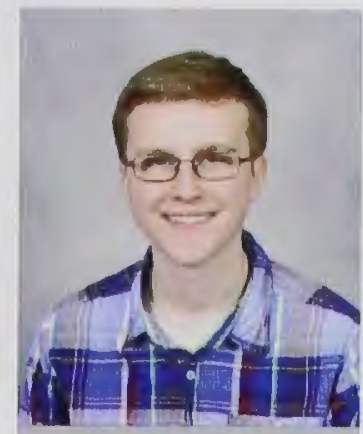

Josiah Parsons

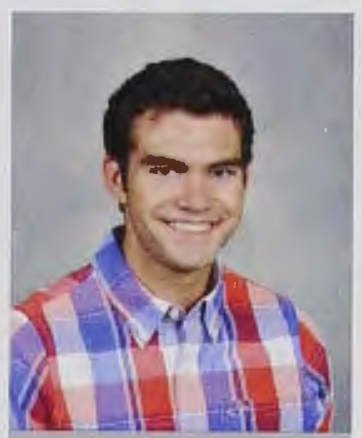

Brent Petersen

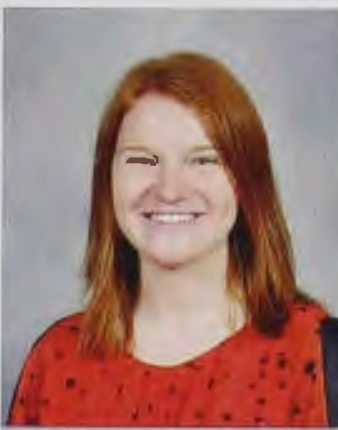

Carolyn Pottle
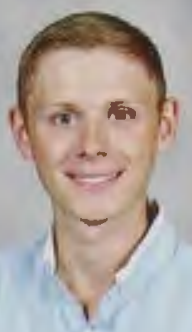

Evan Rayder

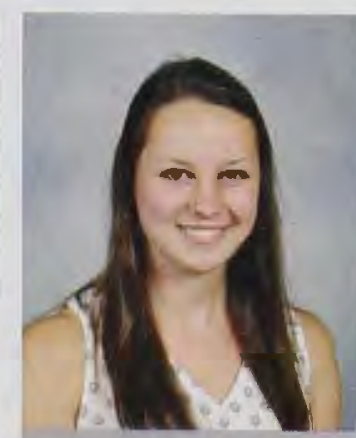

Angela Rosby

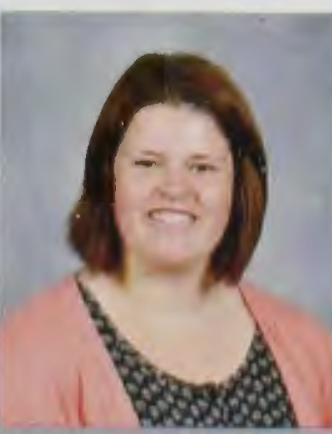

Emily Salch

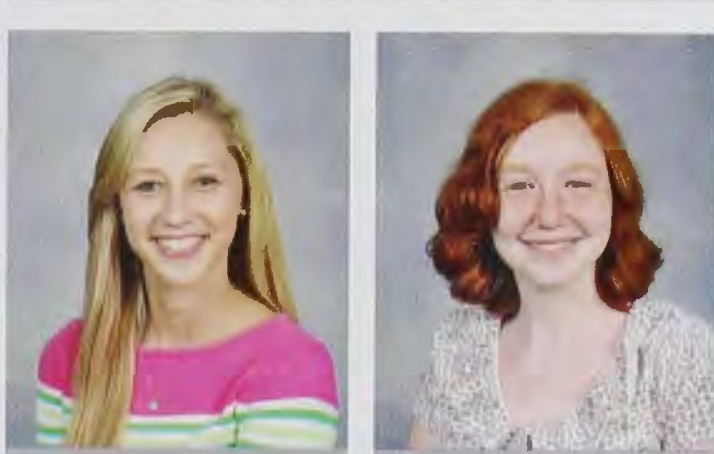

Abigail Rubertus
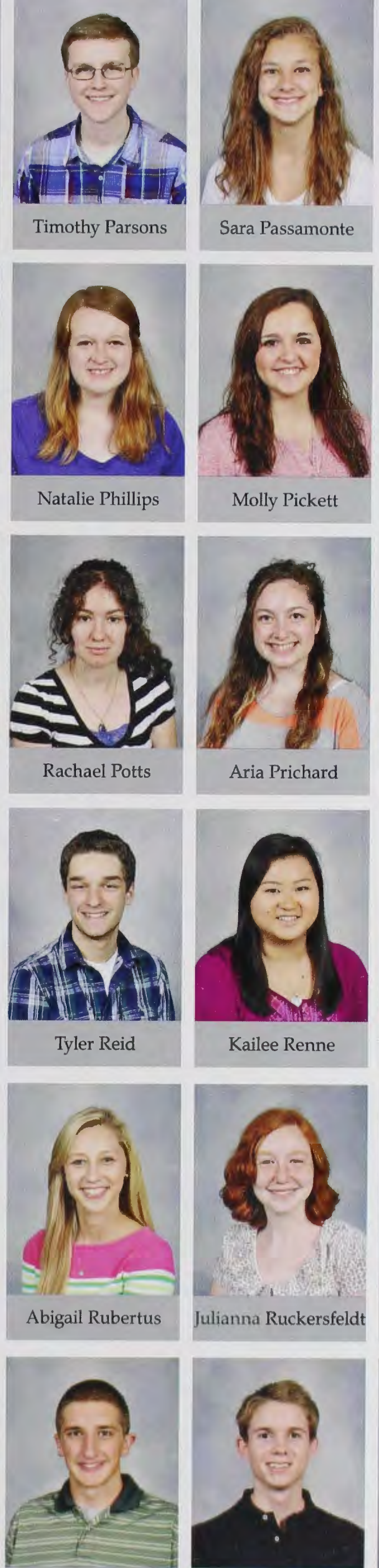

Sara Passamonte

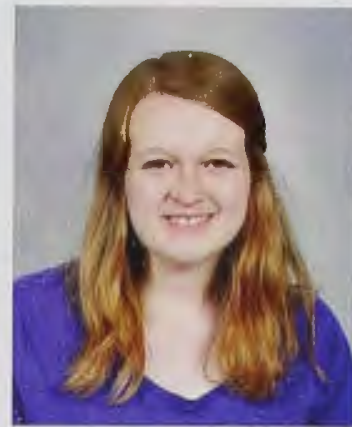

Natalie Phillips

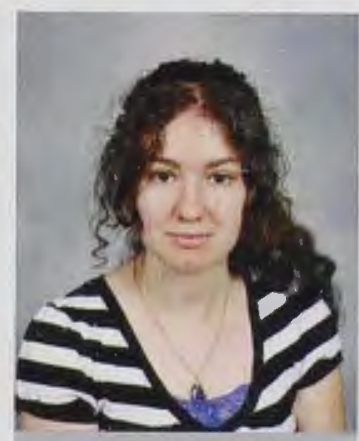

Rachael Potts

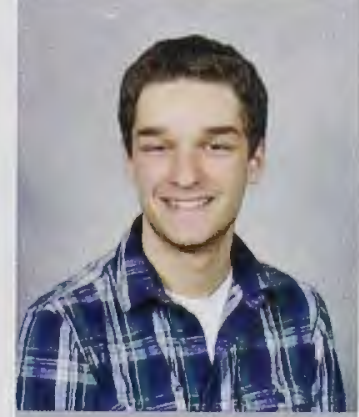

Tyler Reid

Gabriel Sallstrom

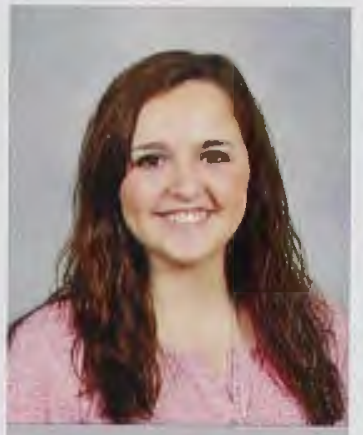

Molly Pickett

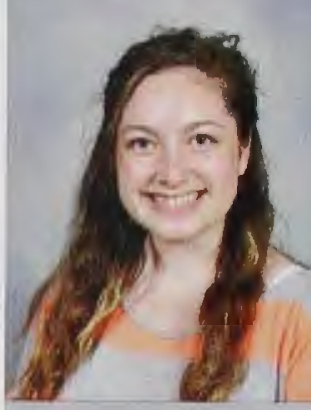

Aria Prichard

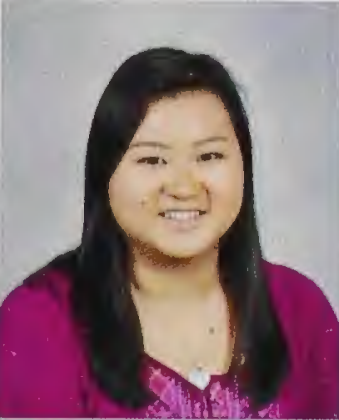

Kailee Renne
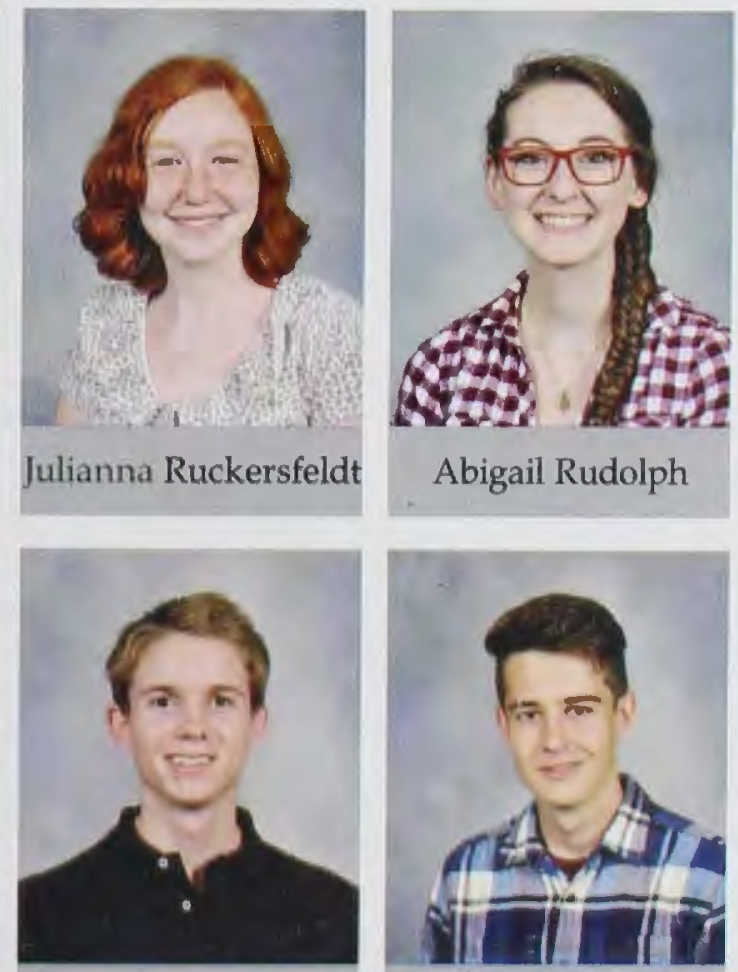

Stephen Samuelsen
Abigail Rudolph

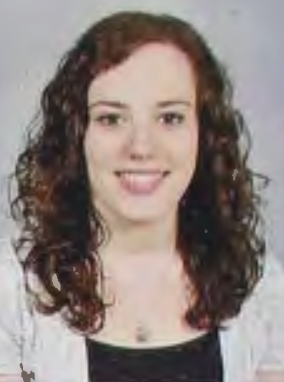

Alexandria Pavlantos

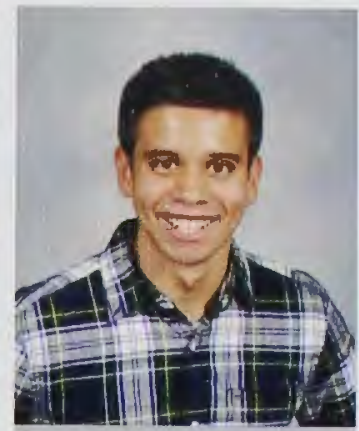

John Piraino

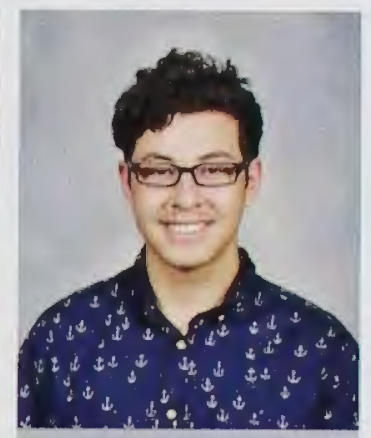

Adrian Quijada

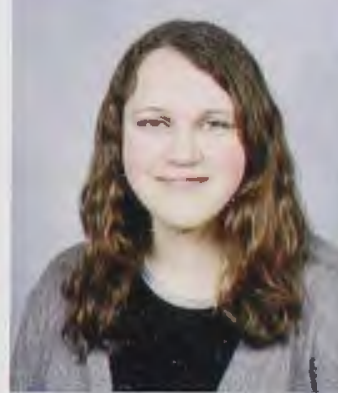

Monica Reyynolds

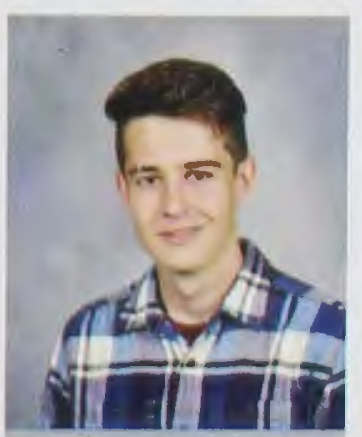

Drayton Santavicca

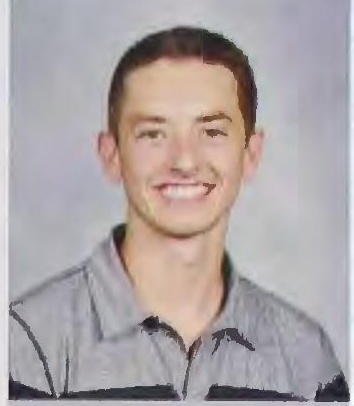

David Pelletier

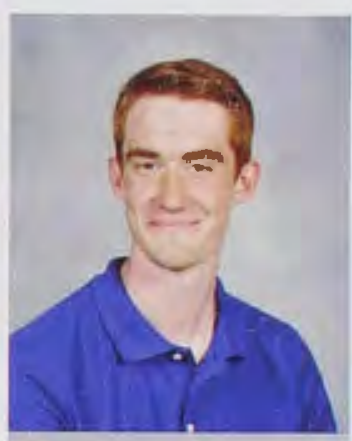

David Pitts

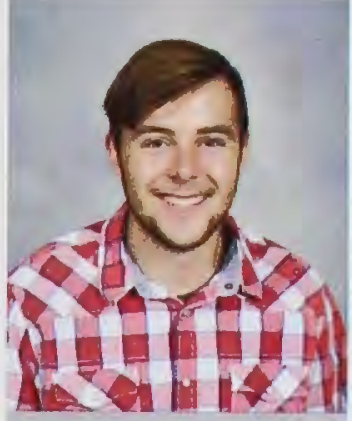

Andrew Rarick

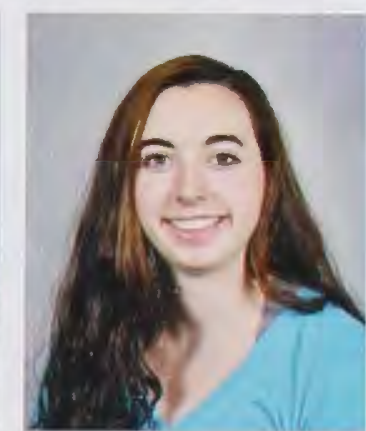

Kaitlyn Ring

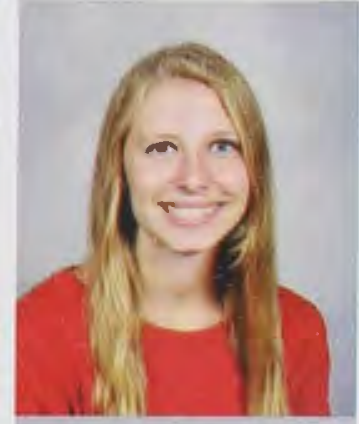

Abigail Ruminski

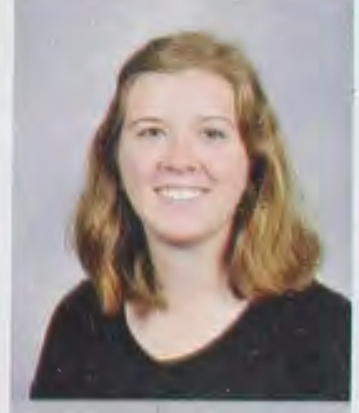

Emily Savard 


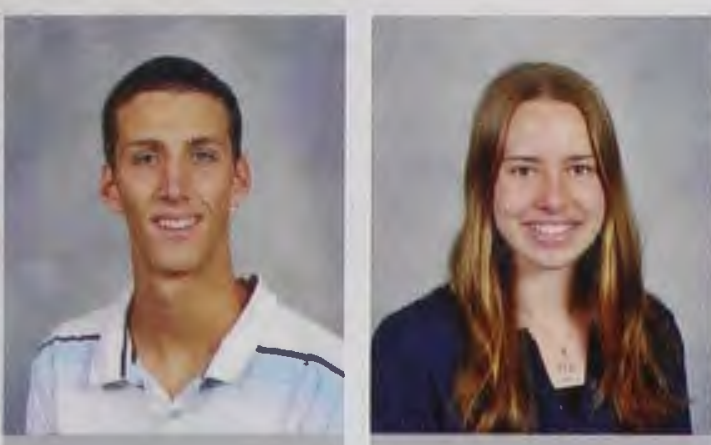

Timothy True

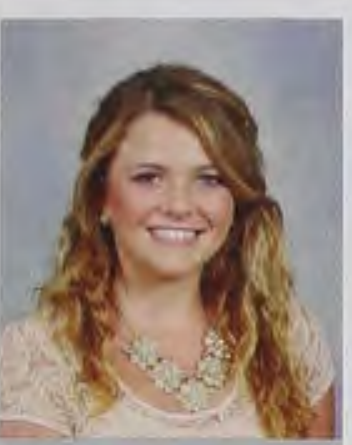

Victoria VanHorn

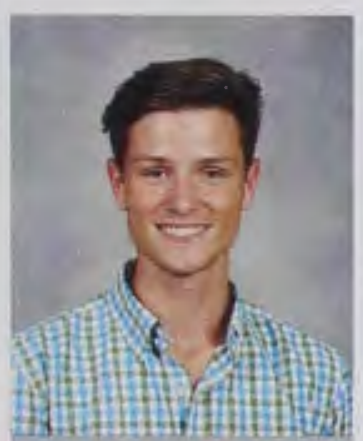

Joseph Vroegop

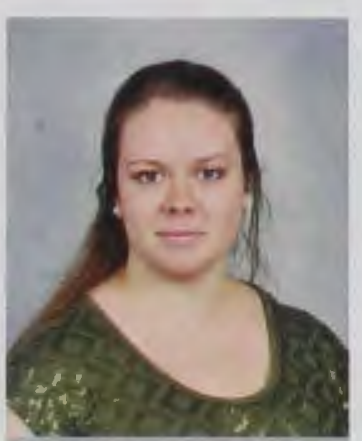

Katrina Weaver

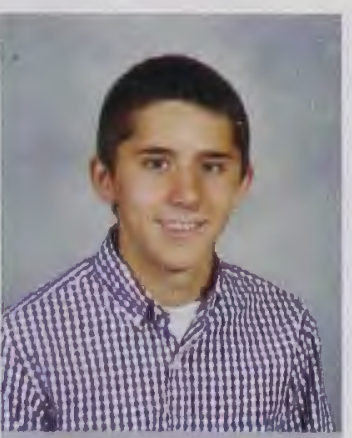

Caleb Williams

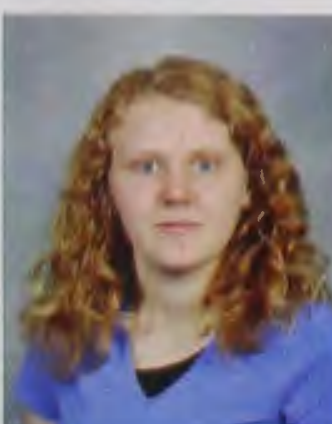

Tineka Witt

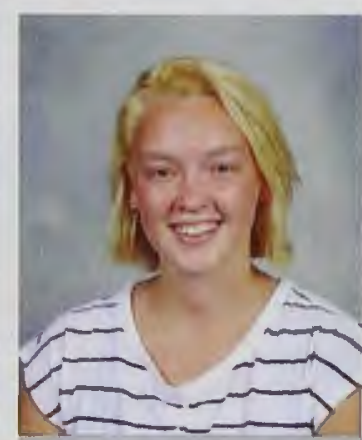

Ashleigh Veltman

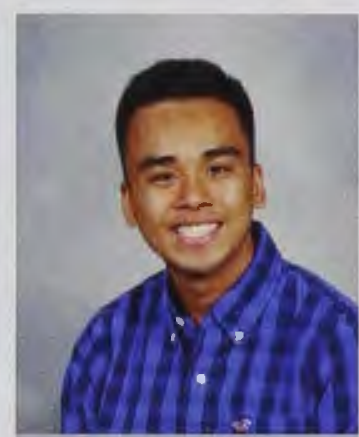

Trieu Vu

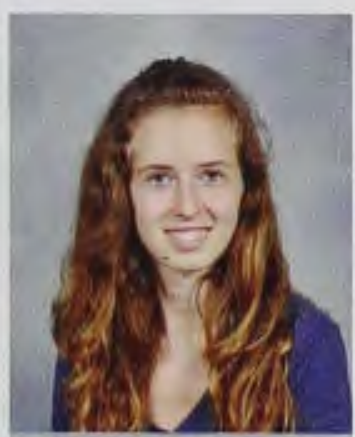

Emma Wessels

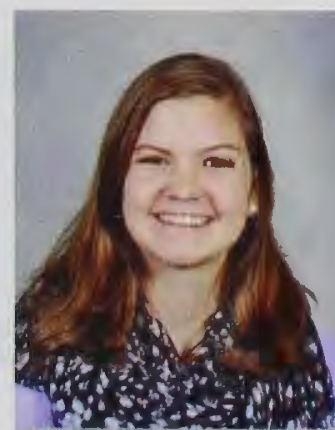

Rebekah Williams

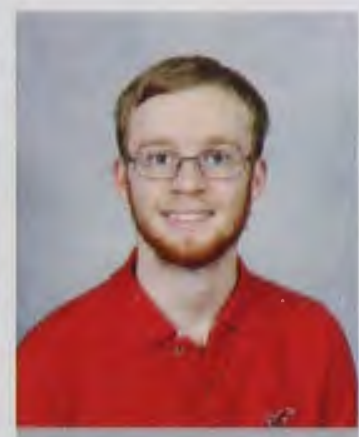

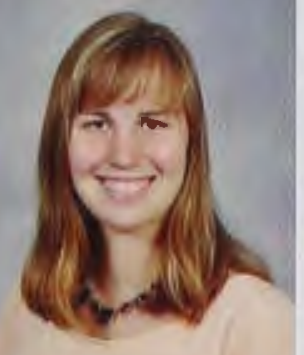

Jennifer Tyson

Joshua Woolverton

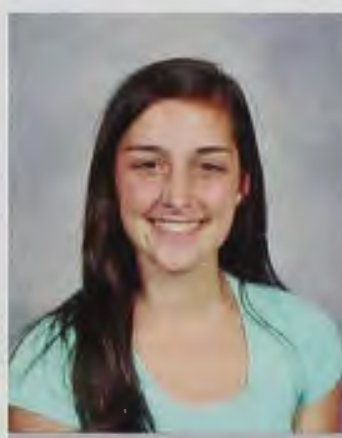

Abbie Vetter
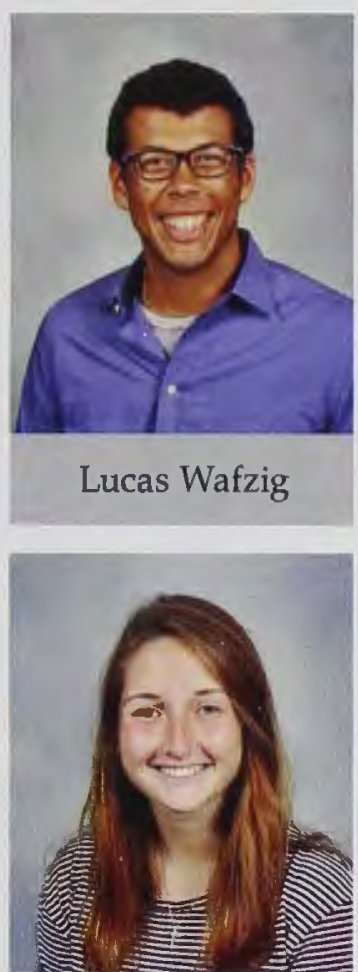

Jessica Westenberg

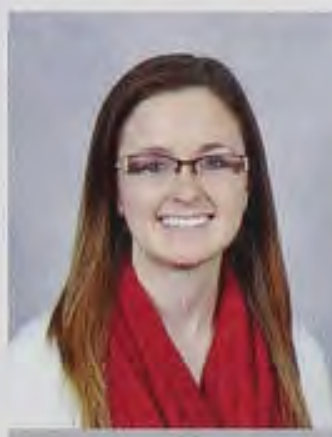

Sarah Williams

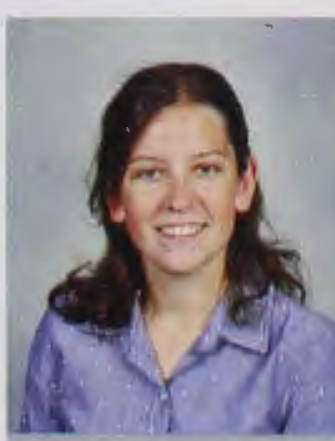

Allie Wright
Lucas Wafzig

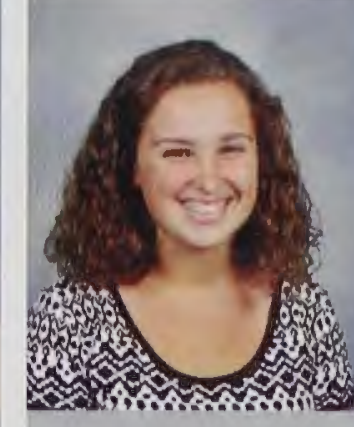

Alison Uhland

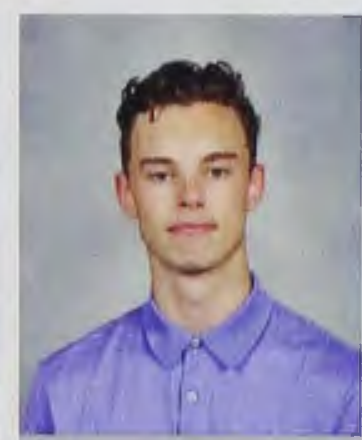

Etienne Viaud-Murat

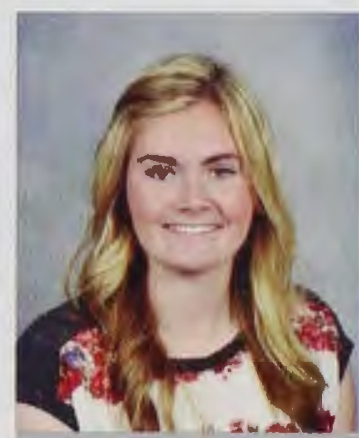

Taylor Waggoner

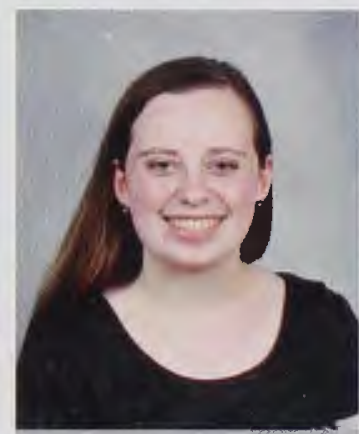

Katelyn Whalen

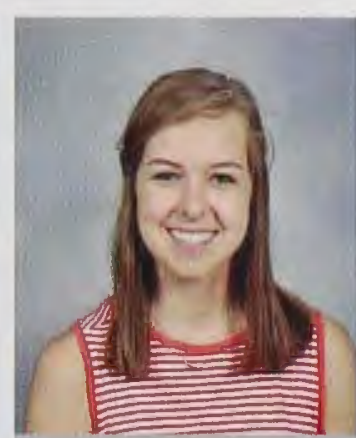

Kelsey Williamson

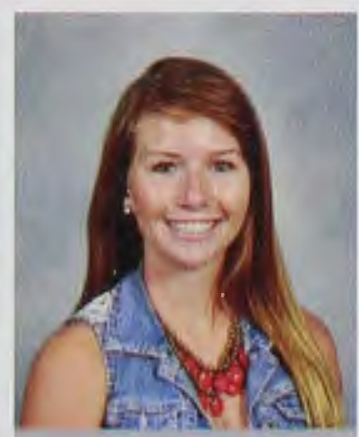

McKenna Wright

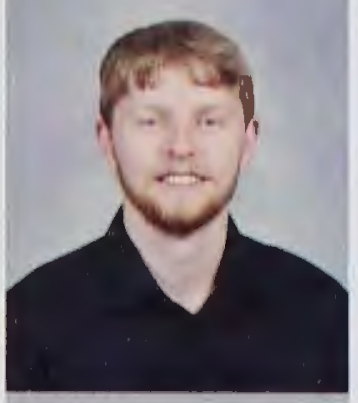

Jonathan Ullom

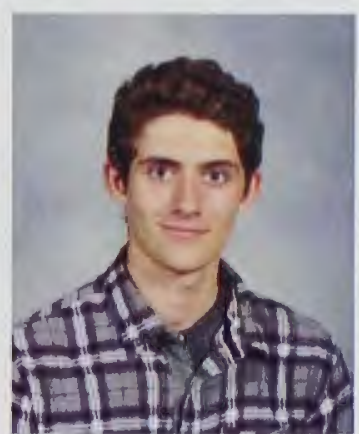

Luke Vincett

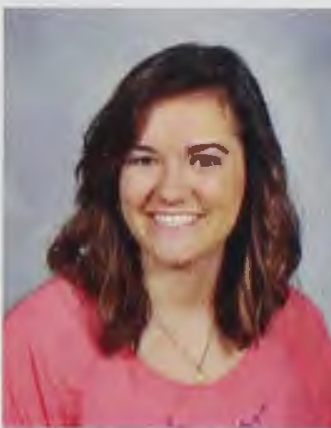

Allyson Wagner

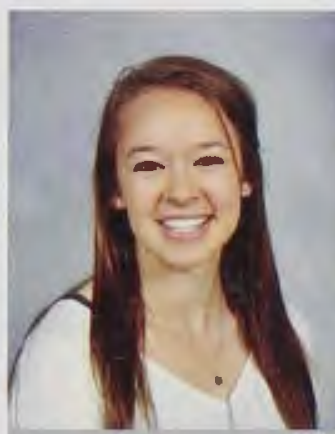

Rebekah Whittles

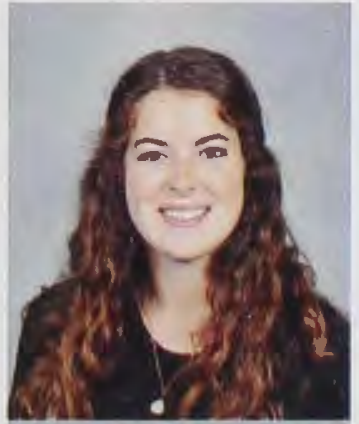

Alisha Willis

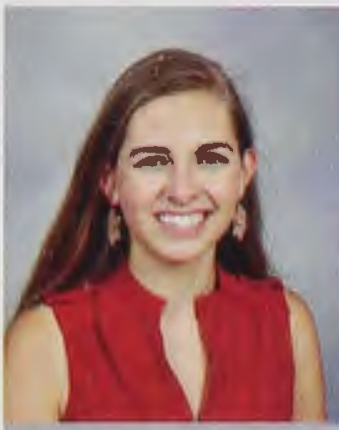

Vanessa Wyn

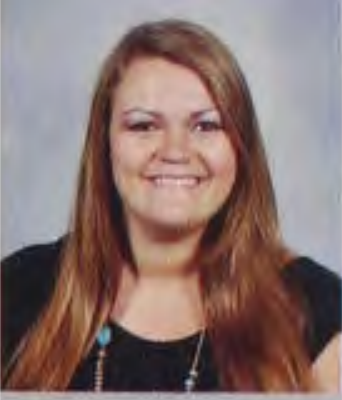

Michelle Van Strien

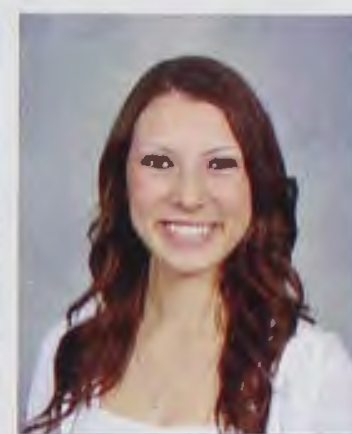

Heidi Vizino

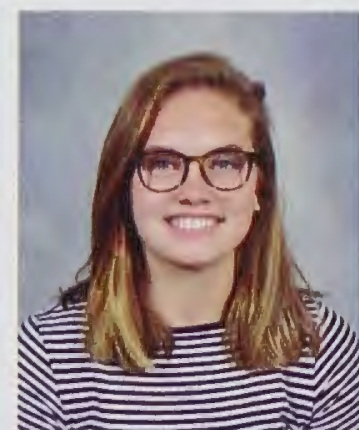

Hannah Ward

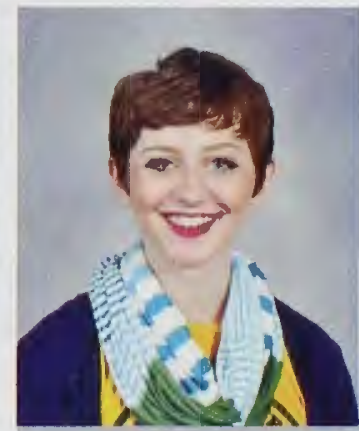

Kimberlyn Wideman

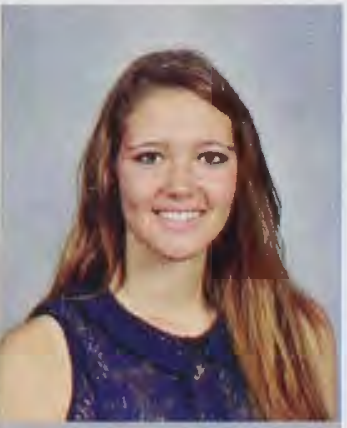

Kristen Wilmot

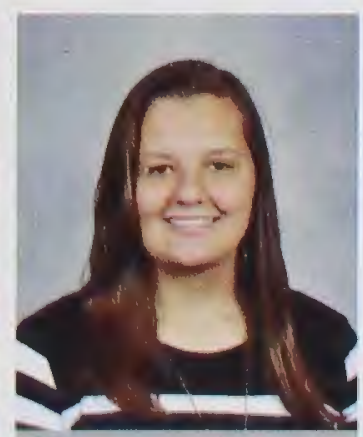

Lane Yoder

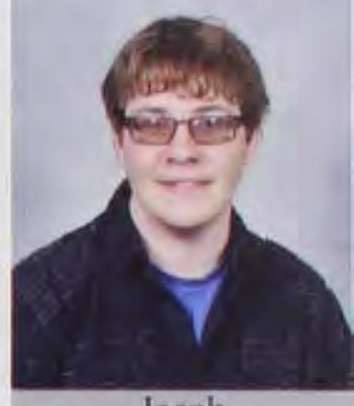

Jacob

Van Veldhuizen

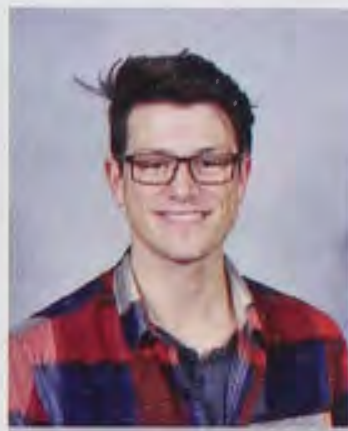

Hayden Vroegop

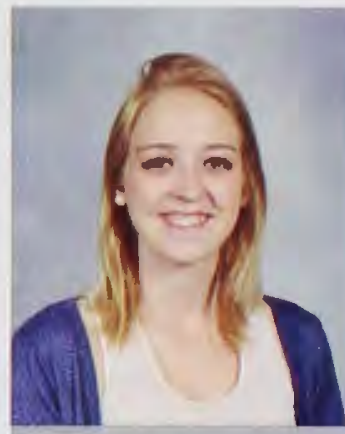

Rebecca Warnshuis

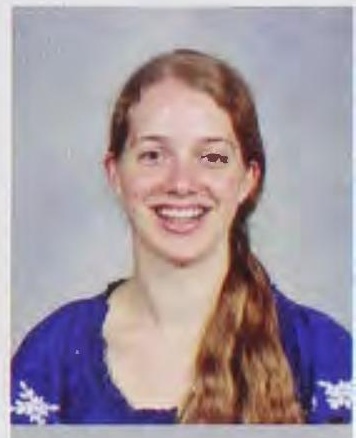

Alicia Williams

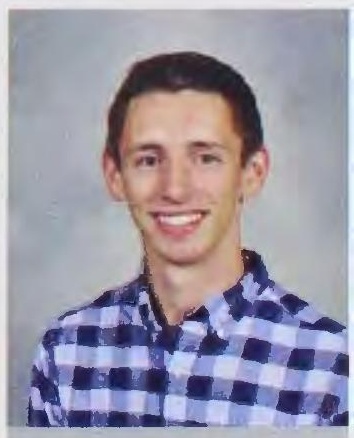

Michael Wilt
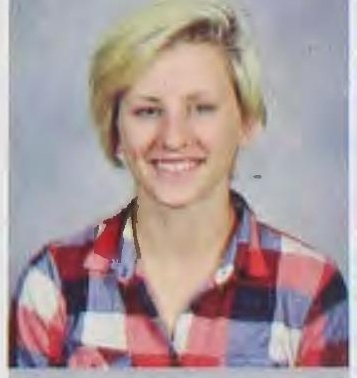

Jennifer Yosinski 
sophomomes

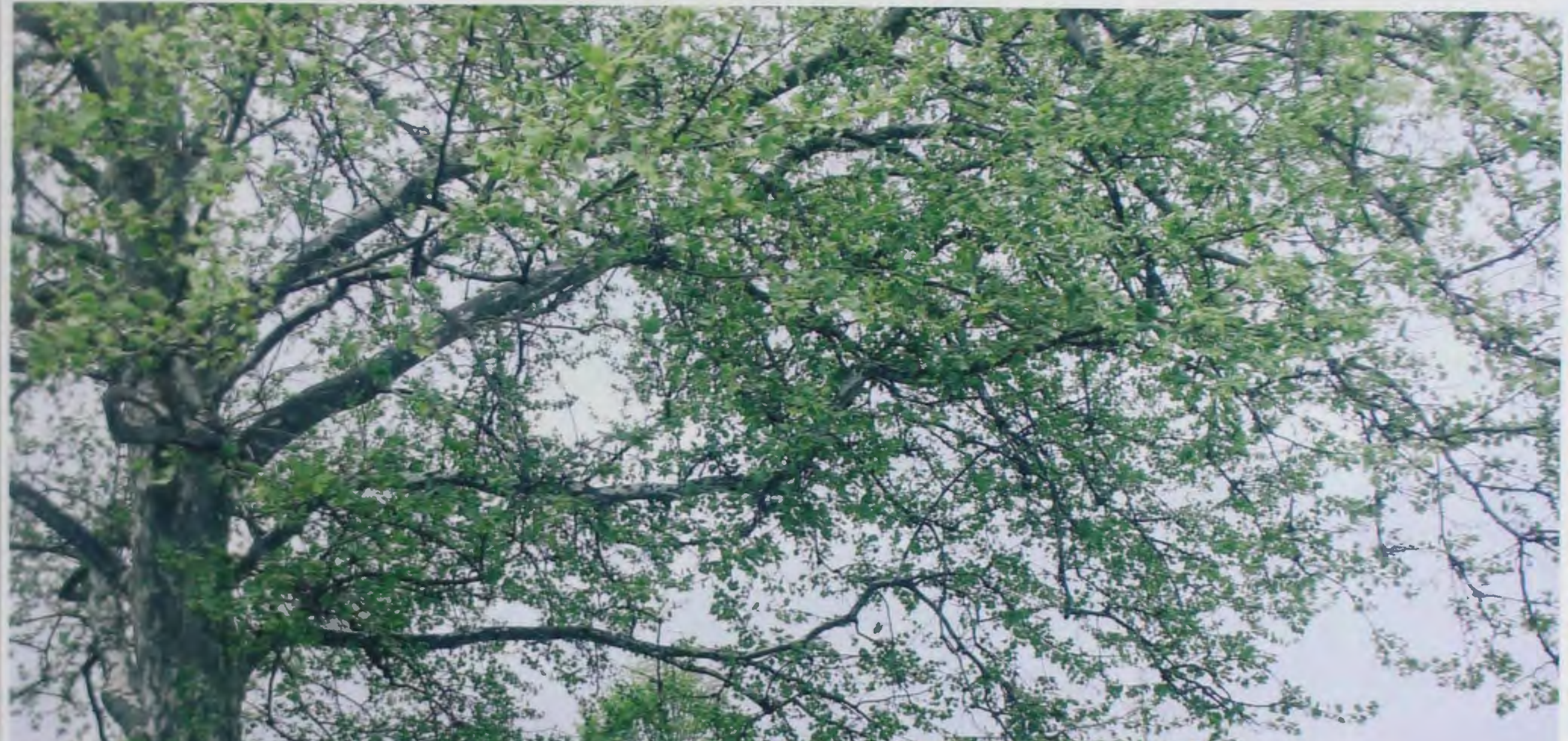

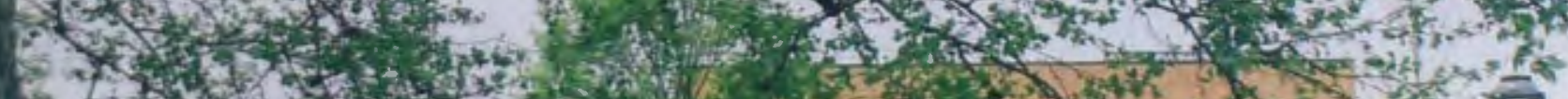

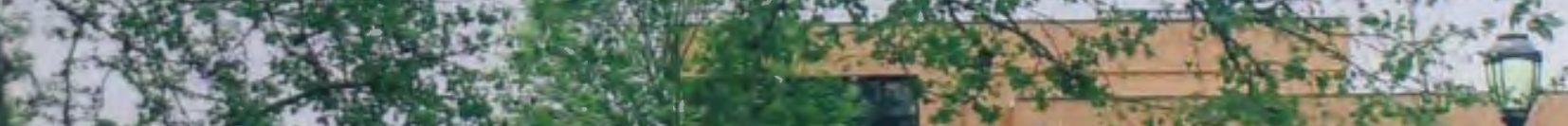

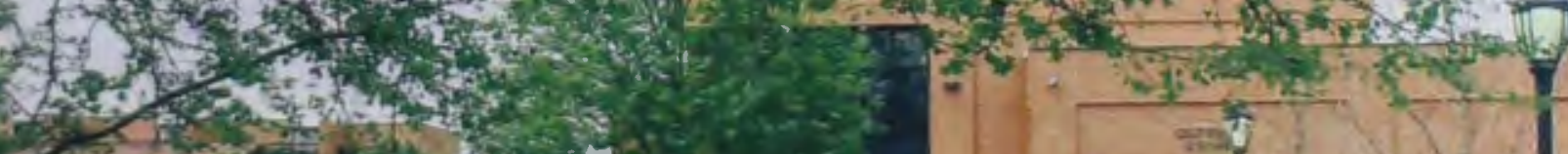
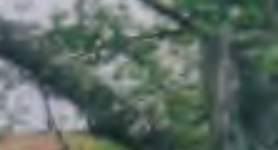
15.
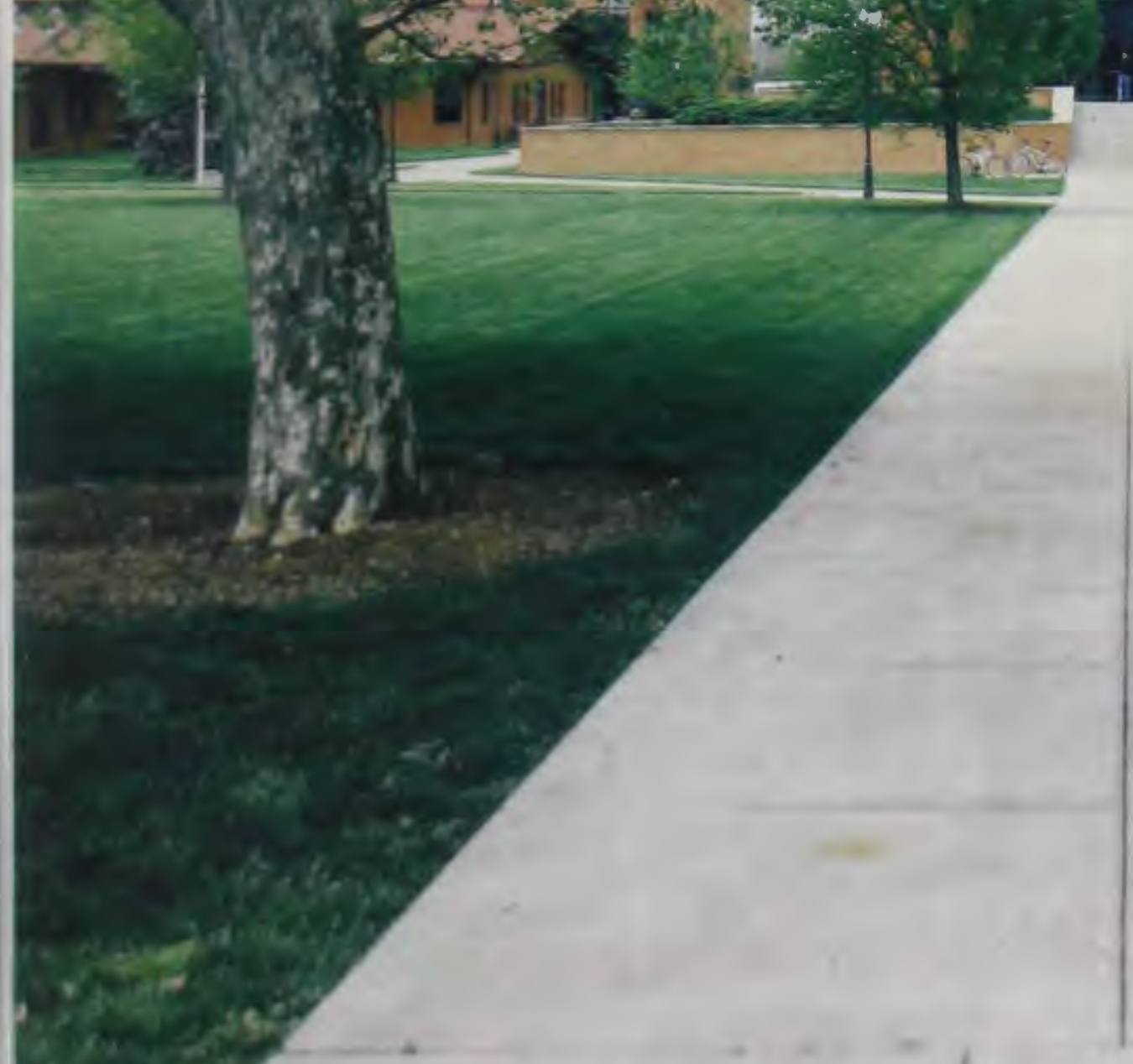
silave
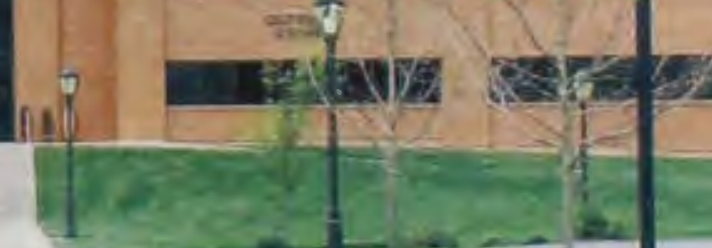


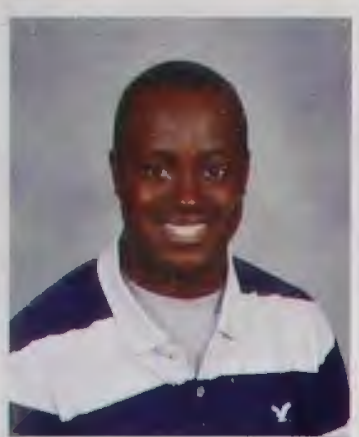

Steven Elizee

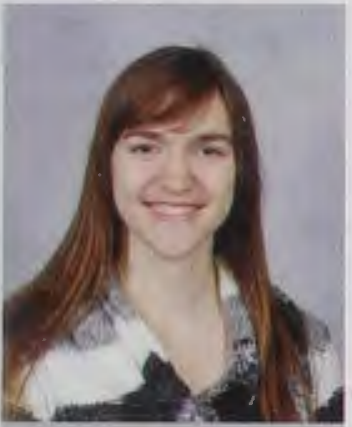

Sara Freeland

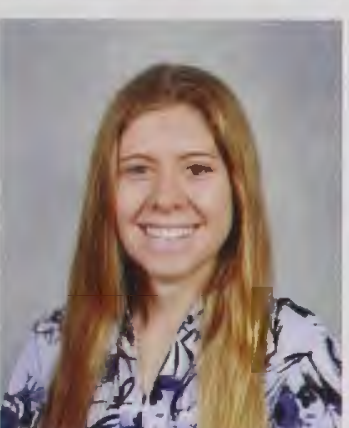

Kaytlin Goodwin
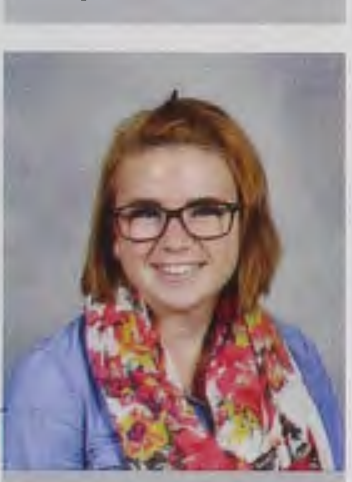

Katelyn Hartsock

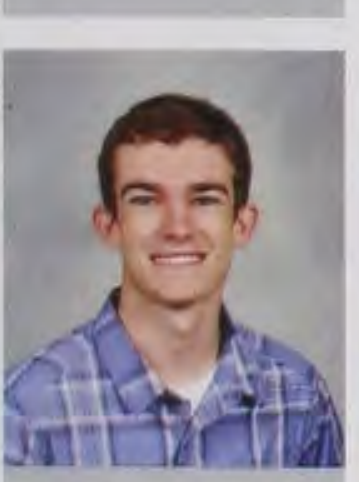

Samuel Herr

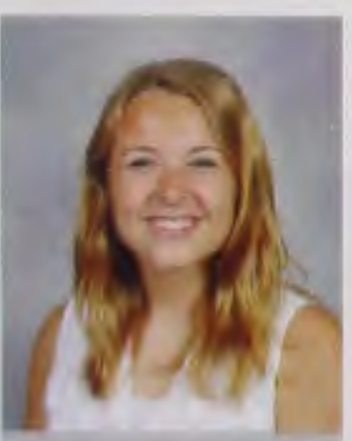

Alyssa Kelly

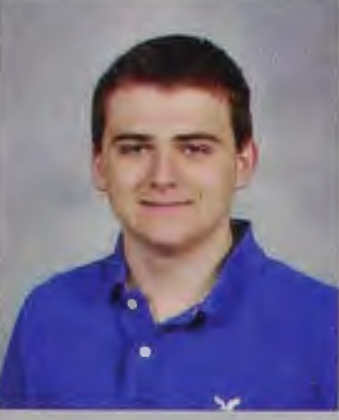

Christian Ellis

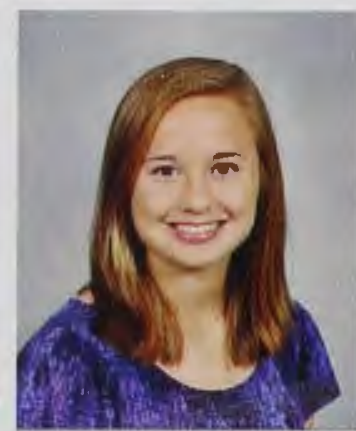

Kjersti Fry

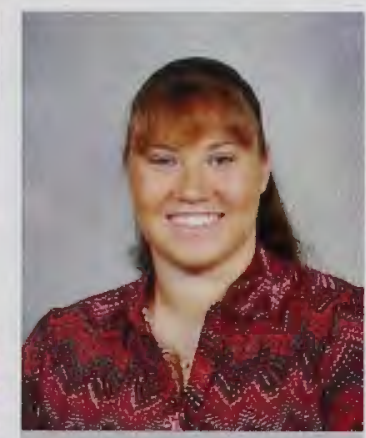

Jenae Gordon

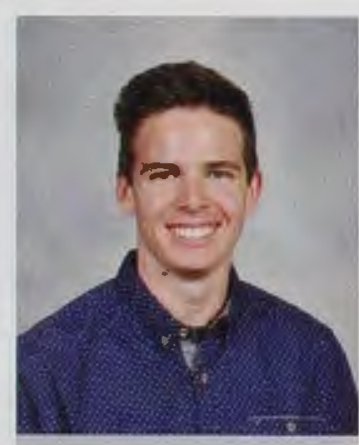

Caleb Hasty

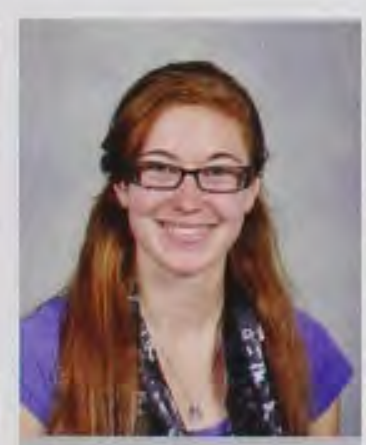

Elizabeth Herting

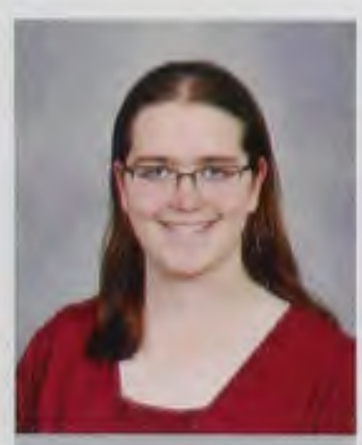

Bethany Khol

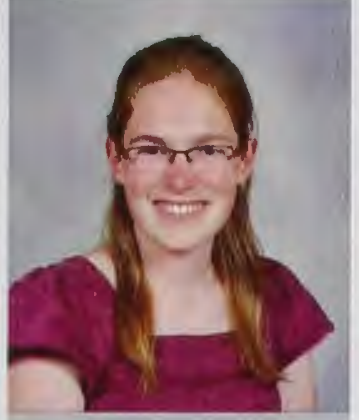

Rebekah Erway

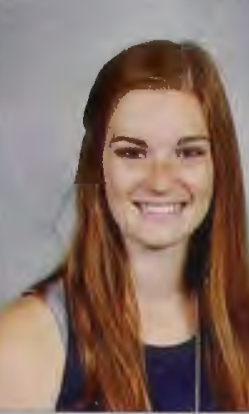

Shannon Garland

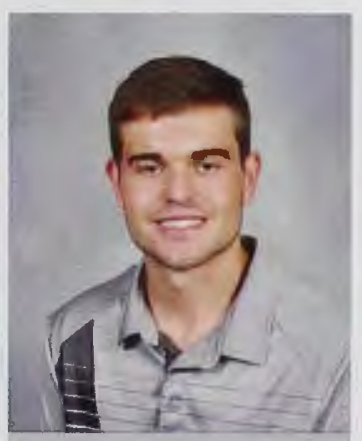

Shad Gordon

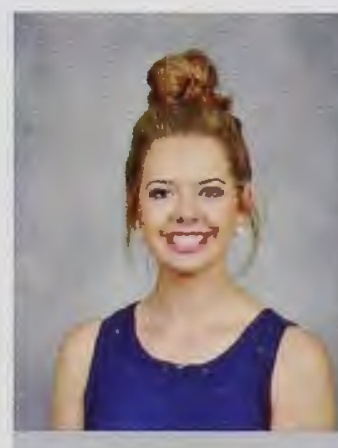

Haley Hauer

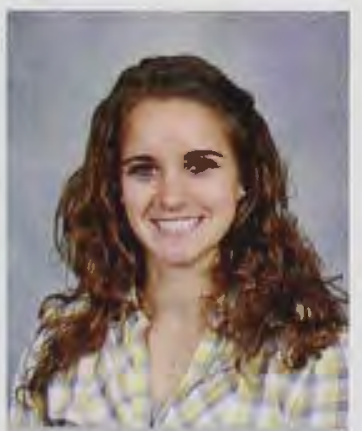

Maggie Huff

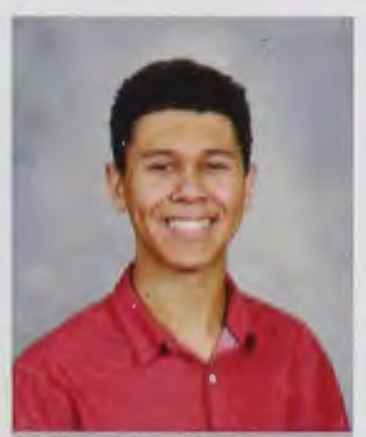

Kyle Kilchrist

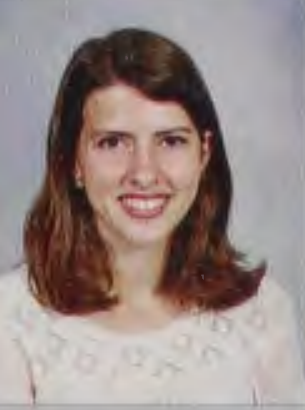

Alanna Esbenshade

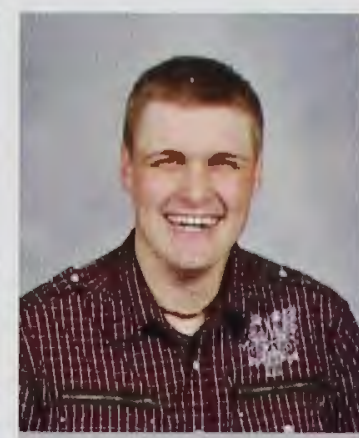

Jacob Gebauer

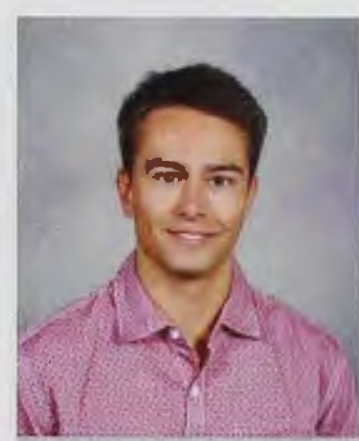

Jacob Grasser

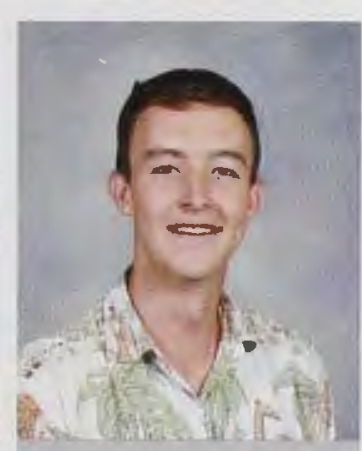

Ryan Hayes

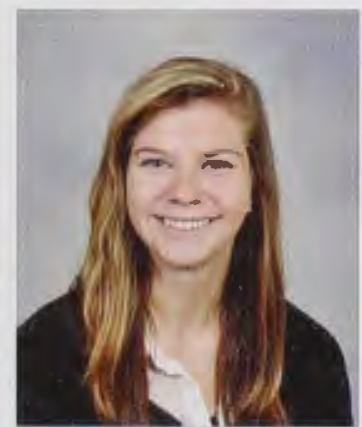

Joy Hughes

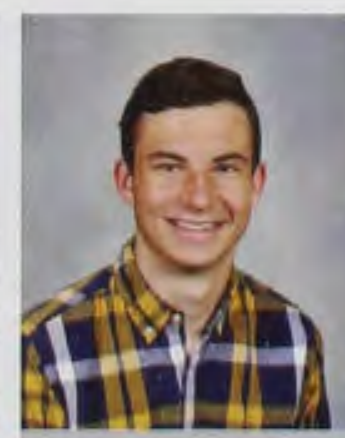

Wesley Kinmel

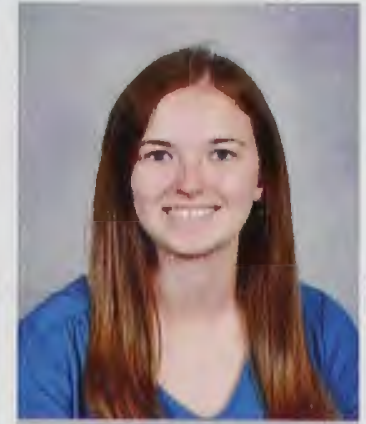

Leah Fisher

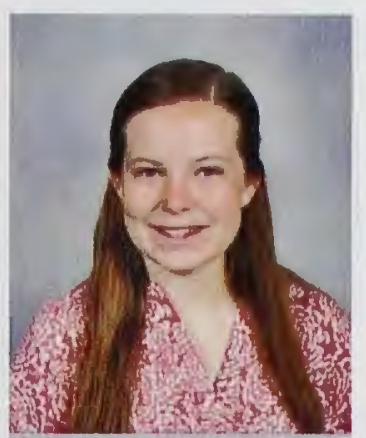

Emily Genet

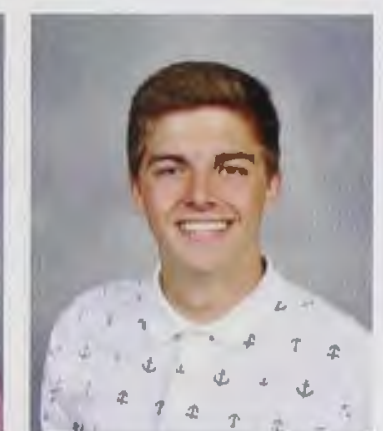

Steven Grout

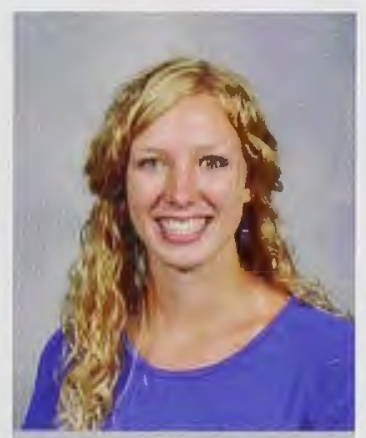

Brienna Hayter

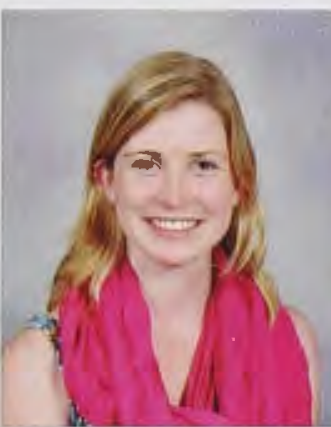

Emily Jenks

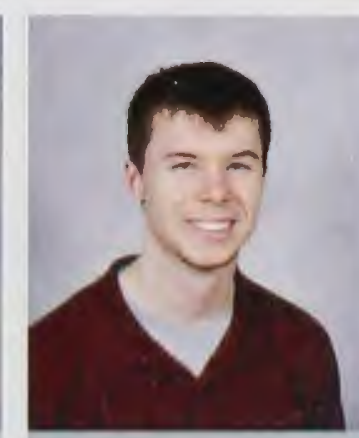

Andrew King

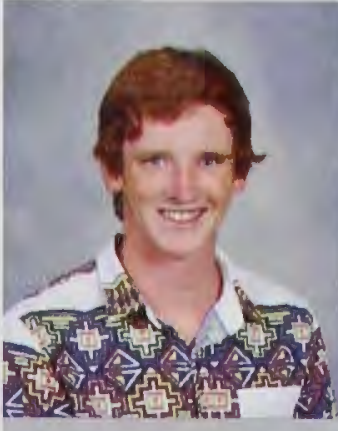

Timothy Flavin

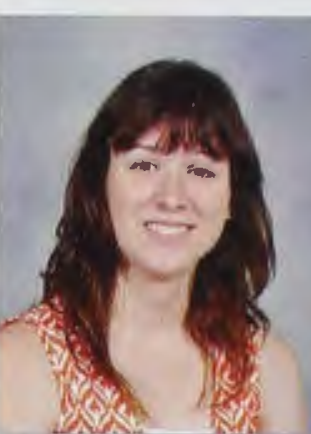

Heidi Gibbs

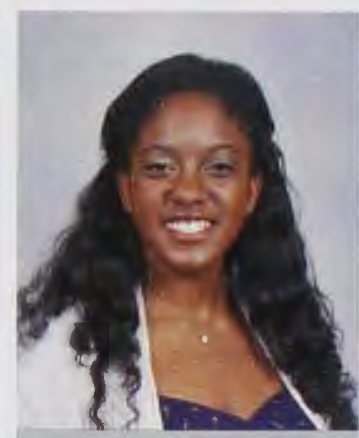

Sharri Hall

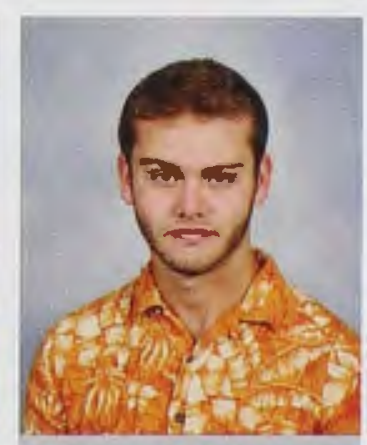

Joshua Heanssler

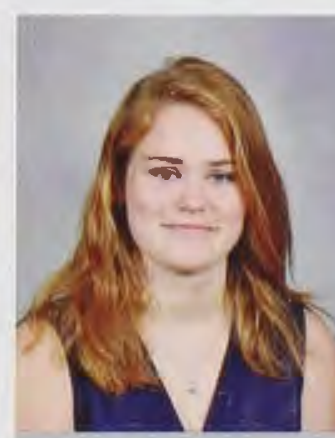

Kassandra Jensen

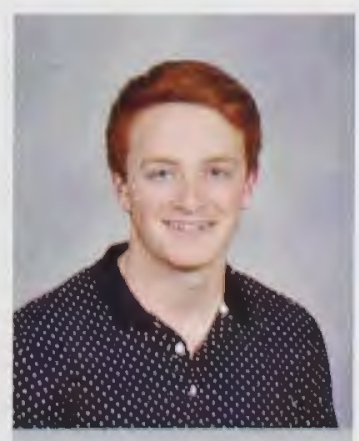

David King

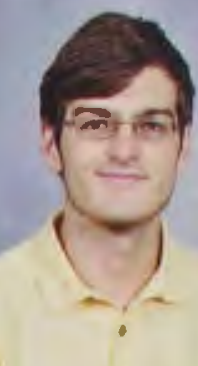

Sean Florer

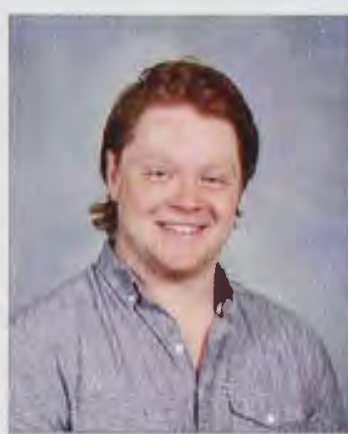

Noah Giles

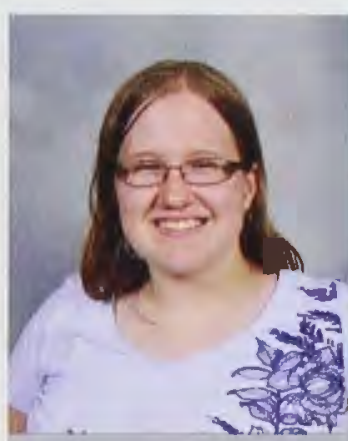

Elizabeth Hansford

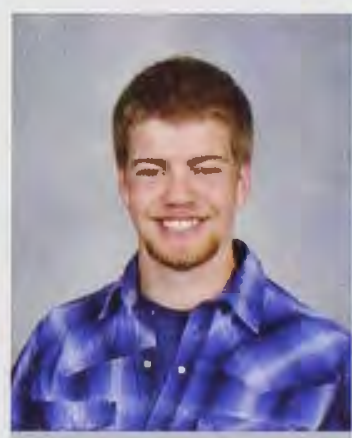

William Heinig

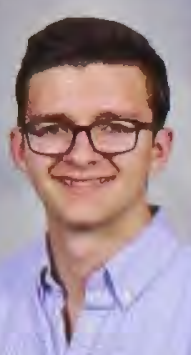

Luke Joy

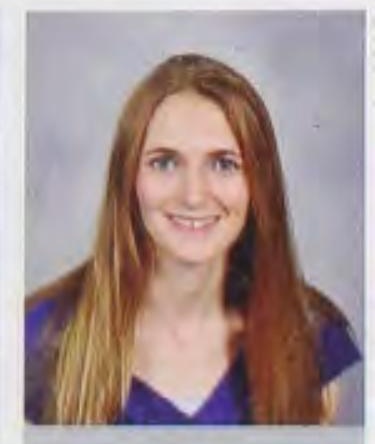

Honor Klassen 


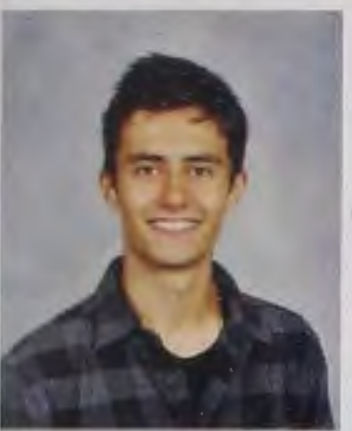

Alexander Paat

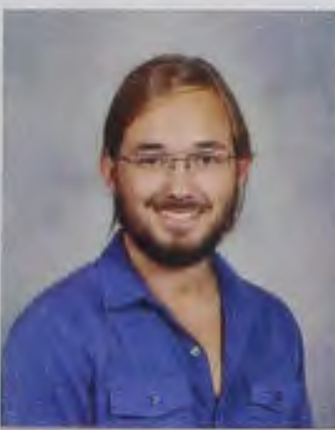

Seth Pierce

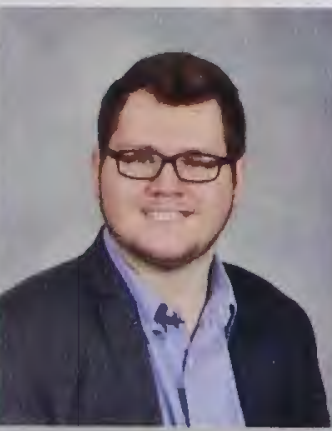

Jacob Reynolds

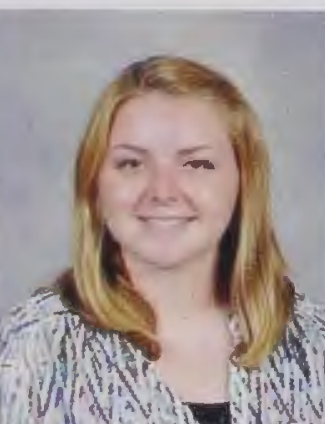

Alivia Rucker

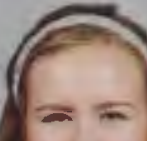

6i) $\Rightarrow 1$

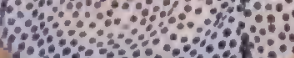

Emily Schmitt

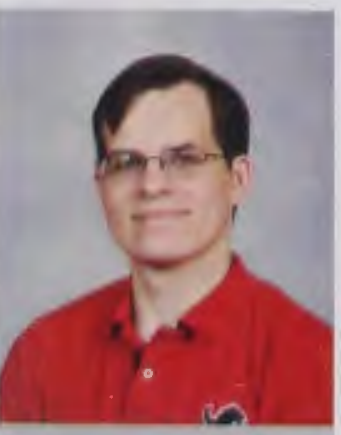

Taylor Shuler

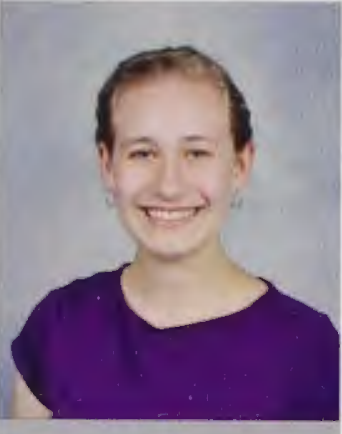

Abigail Patton

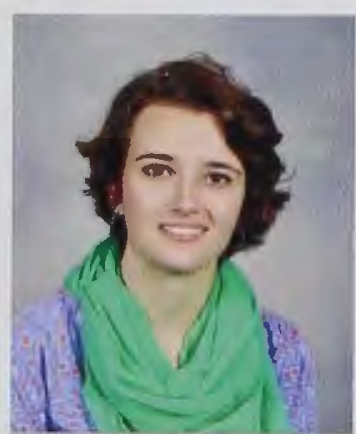

Kristen Pinkerton

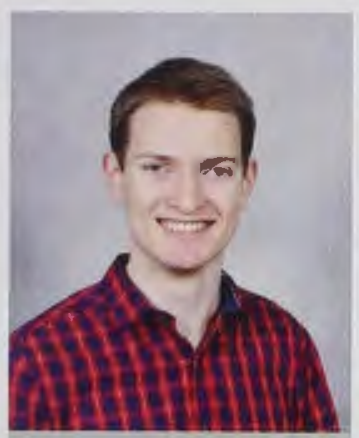

Joseph Richardson

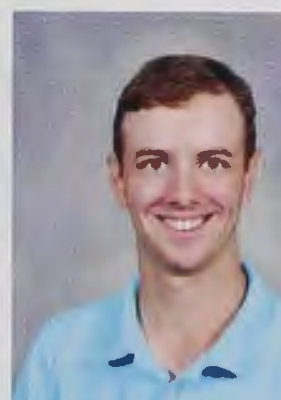

Jesse Russell

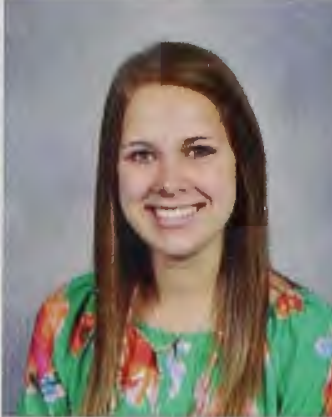

Leslie Pence

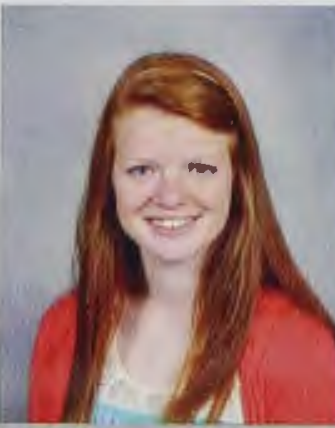

Katelyn Place

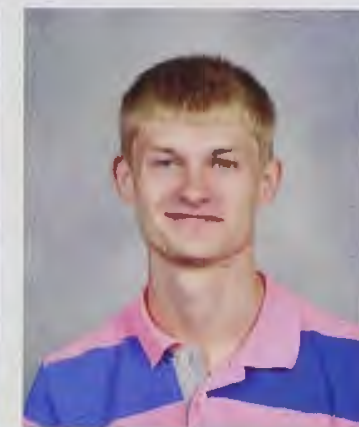

Garrett Rife

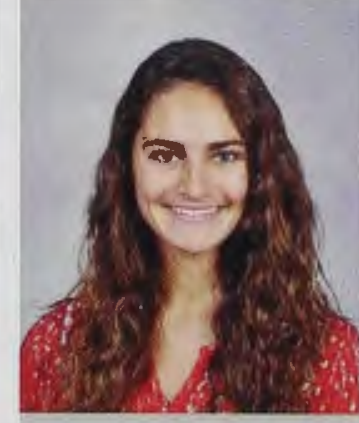

Angelina Sabroso

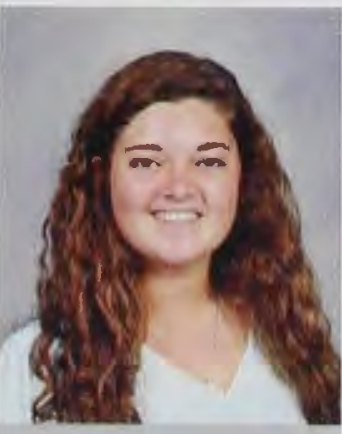

Sarah Schopps

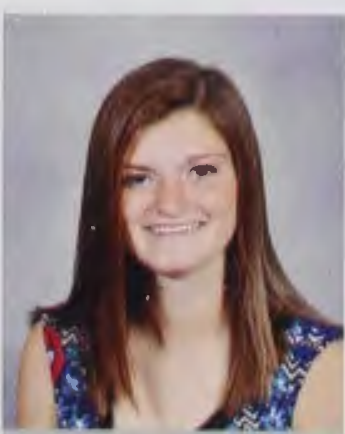

Illyssa Smith

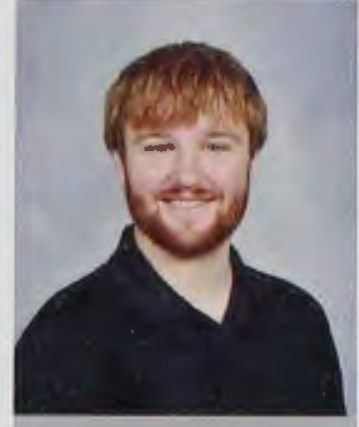

Drake Pensworth

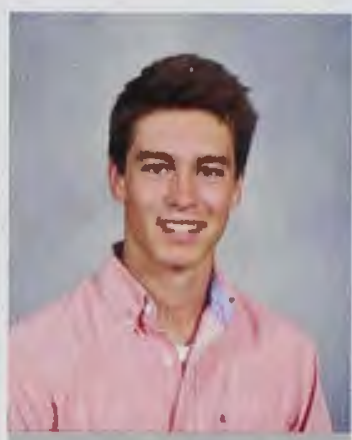

Stephen Proulx

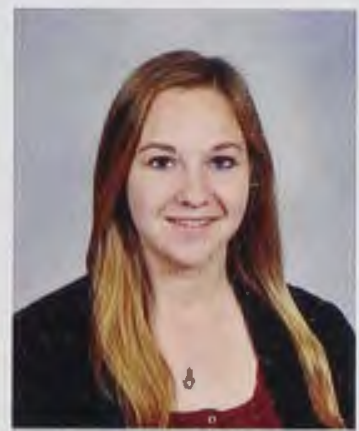

Allison Roberts

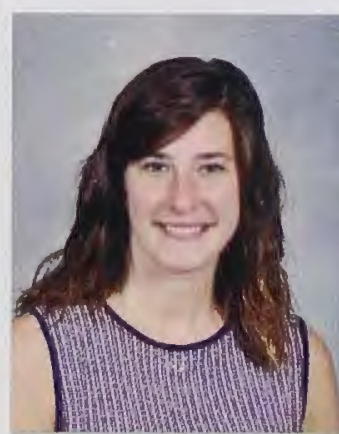

Ashlee Sager

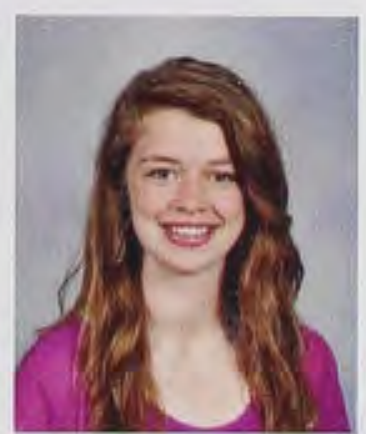

Amy Searl

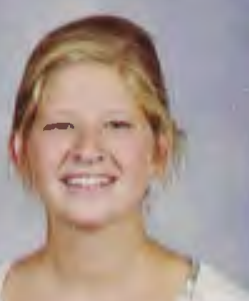

Katie Smith

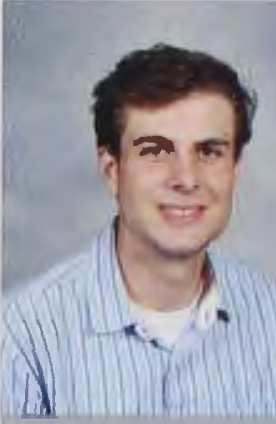

Michael Pereira

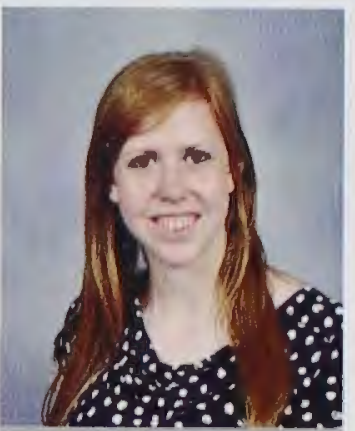

Kayla Ramsey

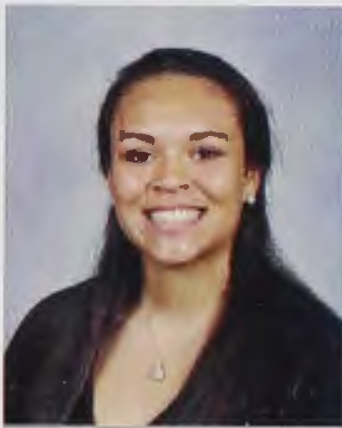

Alexus Roeller

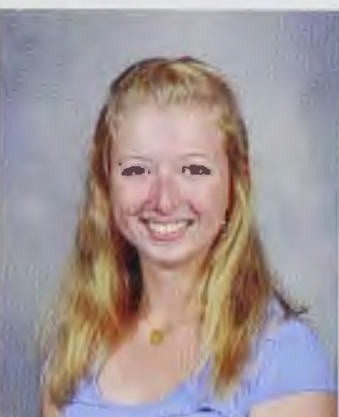

Anna Salisbury

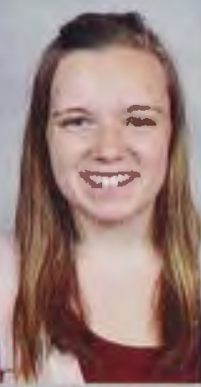

Makayla Segard

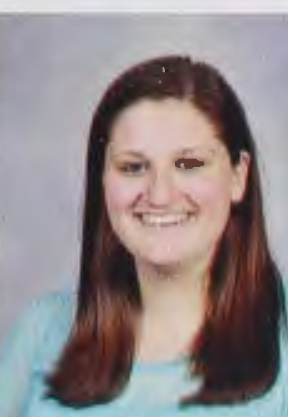

Marissa Smith
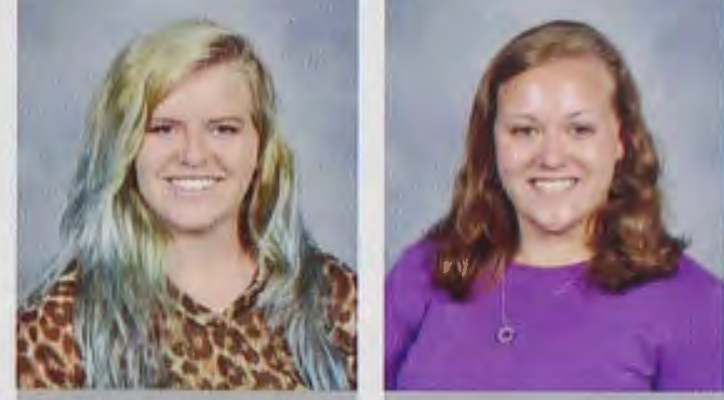

Tasha Peterson

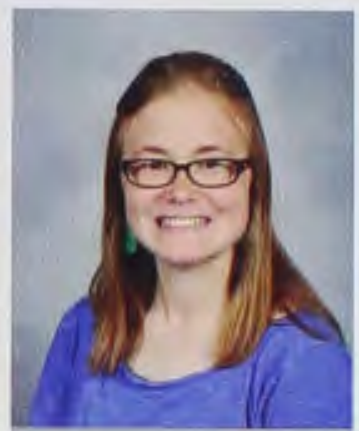

Eleanor Raquet

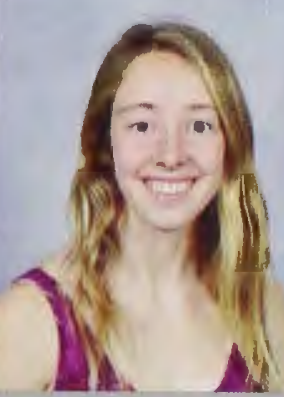

Carly Rose

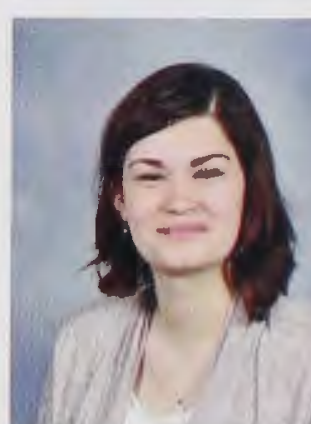

Lindsay Schell

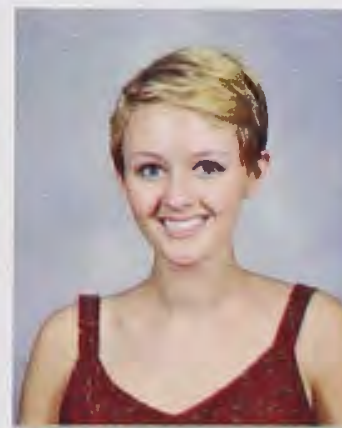

Kristen Shaffer

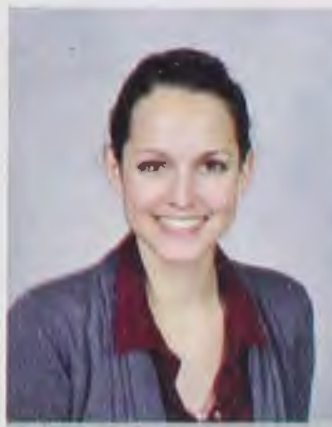

Karolyn Sobo

\section{Hannah Phillips}

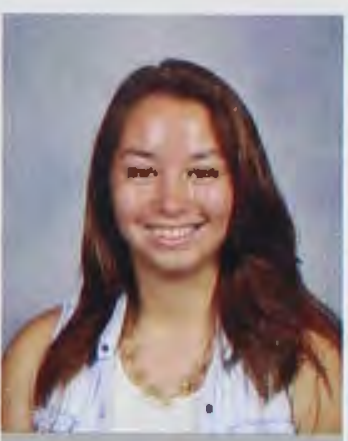

Elyssa Reyes

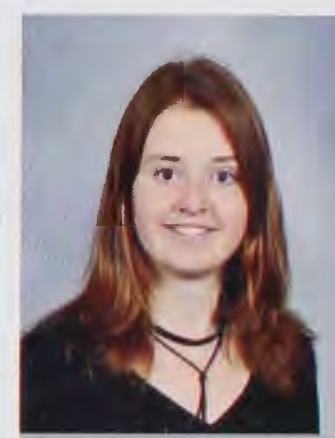

Sarah Rouse

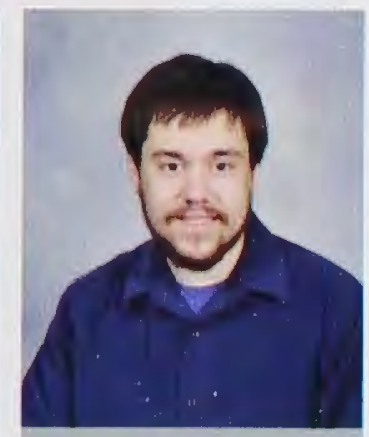

Jules Schieferstein

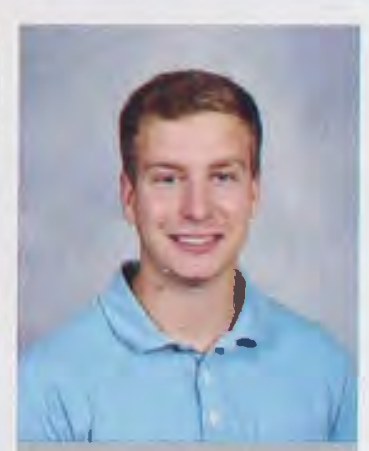

Peter Shields

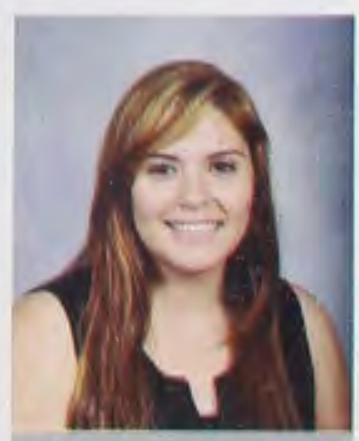

Reina Soczka 

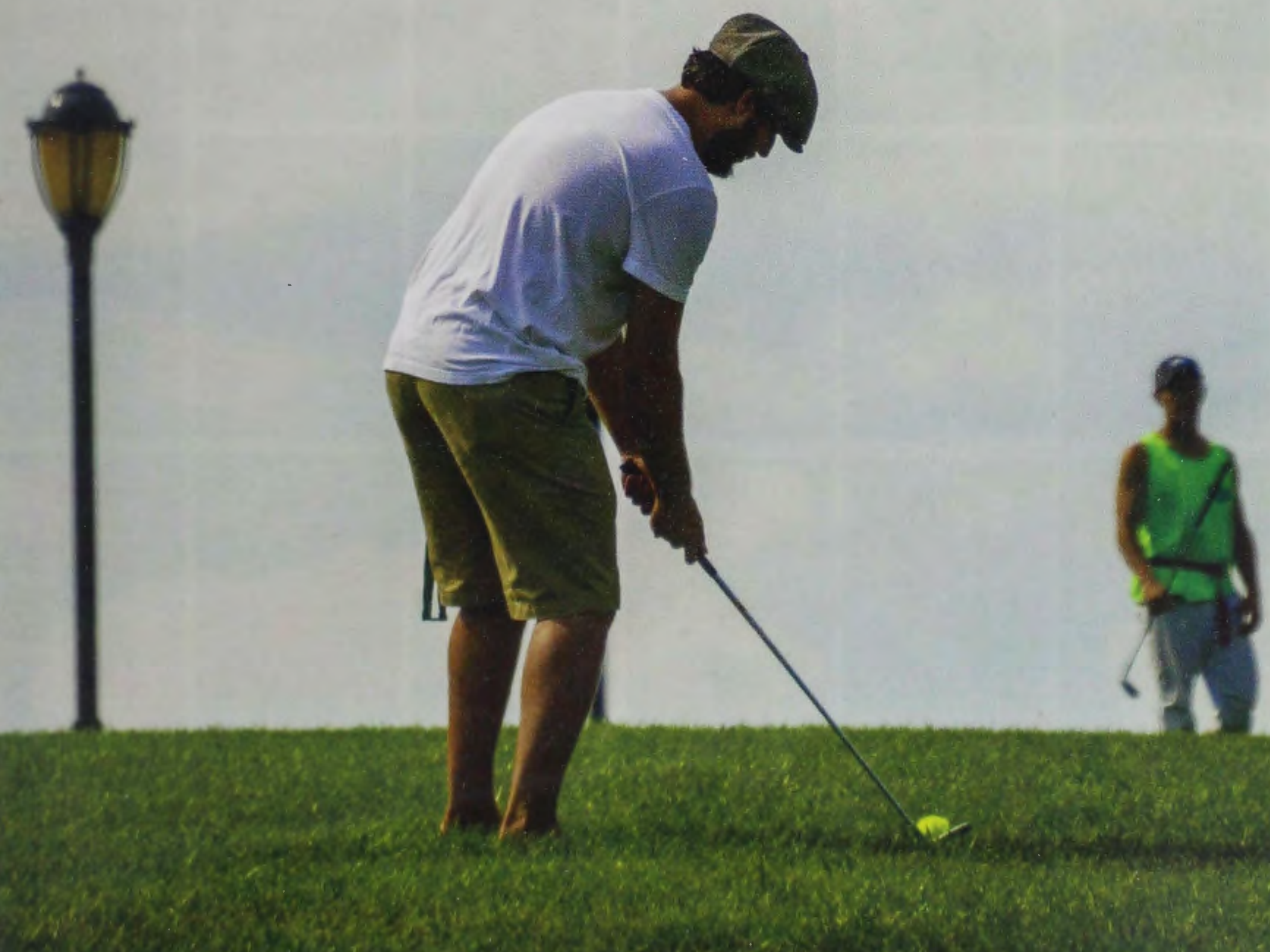

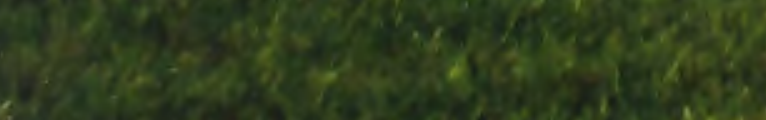
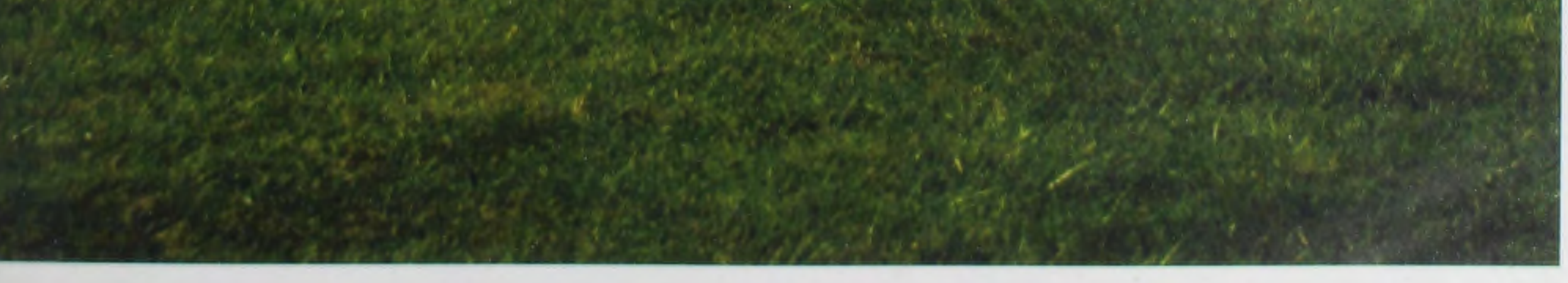


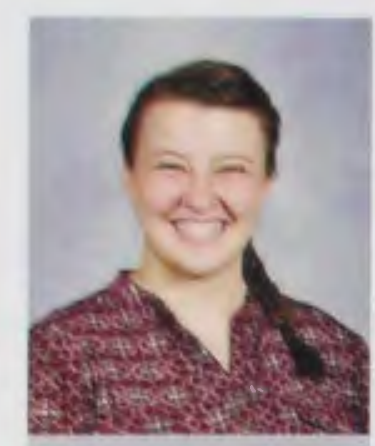

Elizabeth Adams

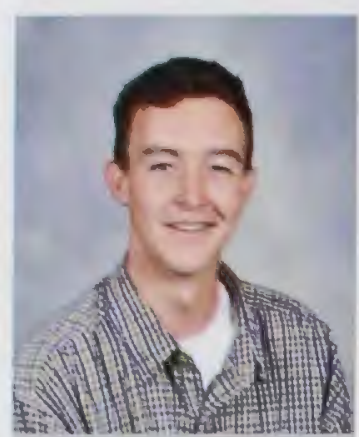

True Baldwin

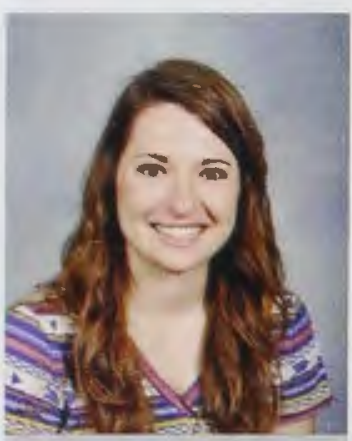

Lauren Bergeron

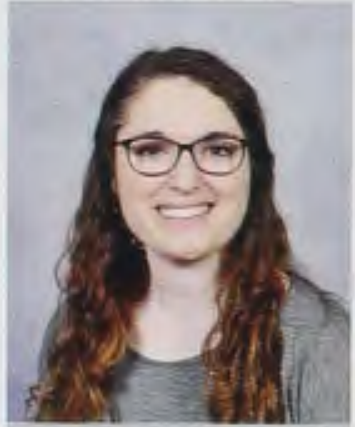

Katelyn Byram

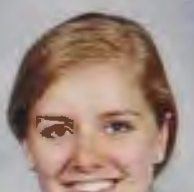

$1-1$

Olivia Clark
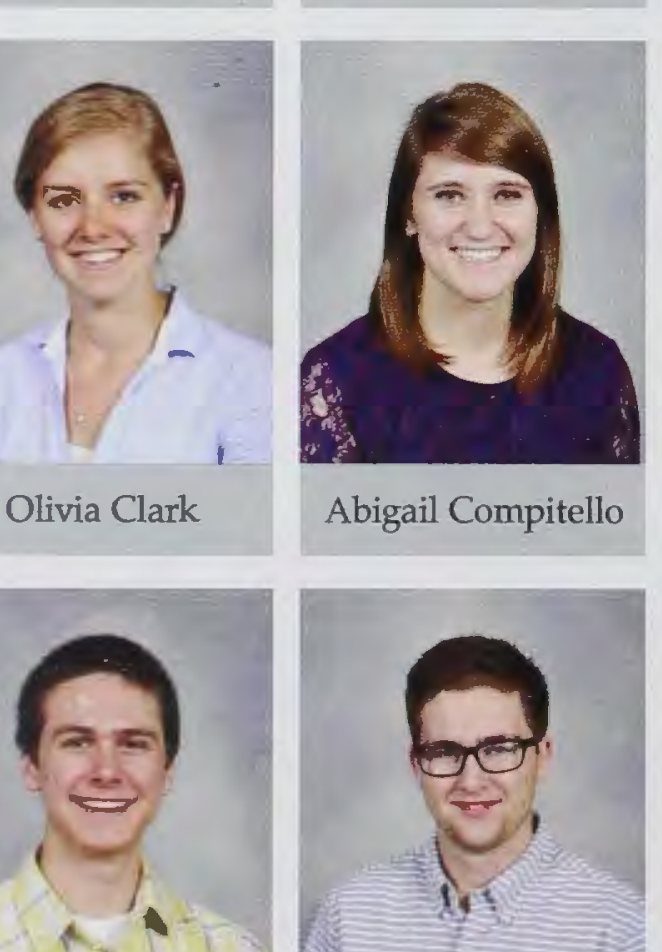

Grant Dennison
Abigail Compitello
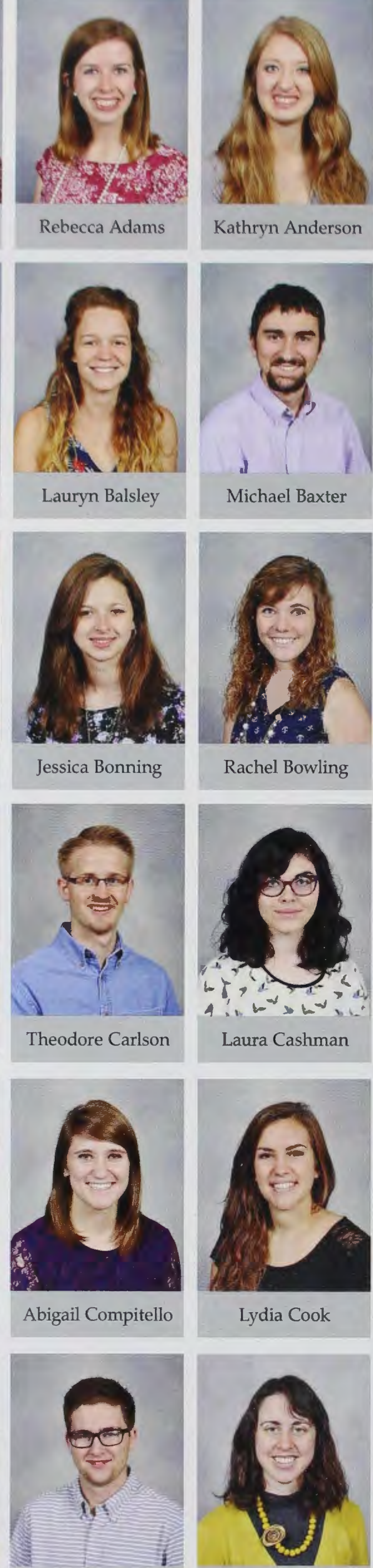

Kathryn Anderson

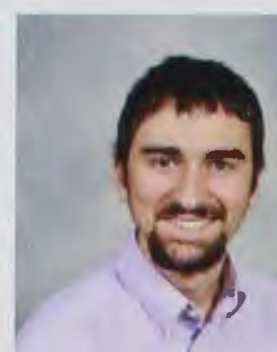

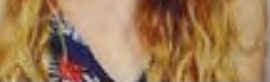

Lauryn Balsley

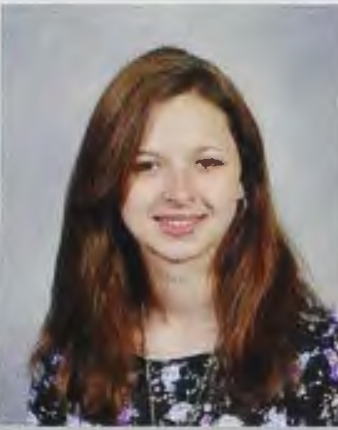

Jessica Bonning

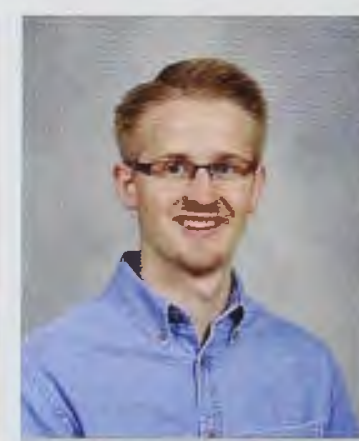

Theodore Carlson

James Dunlap

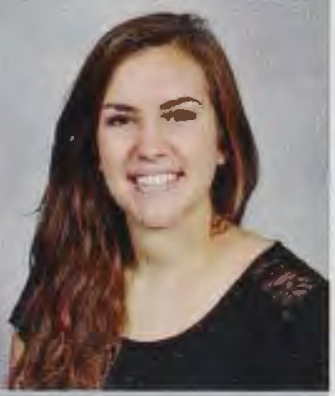

Lydia Cook

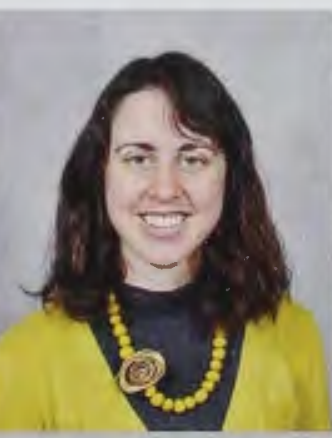

Rebecca Ehnert

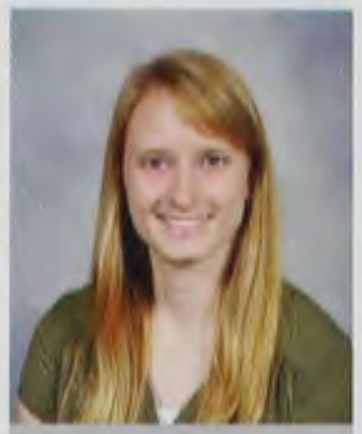

Jill Arthur

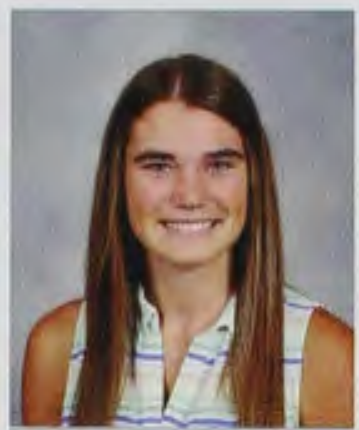

Ashley Beals

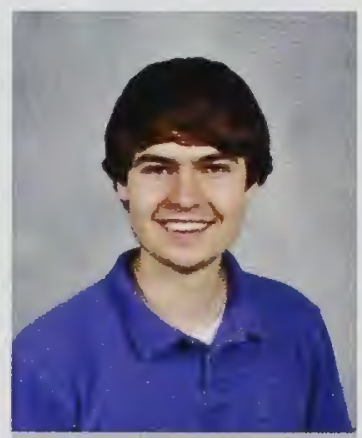

Christopher Brawley

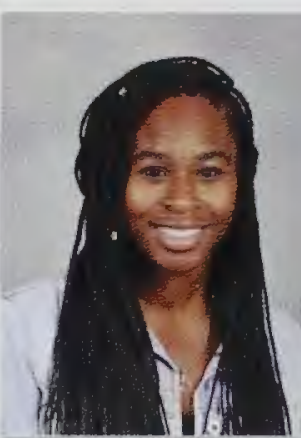

Teara Caston

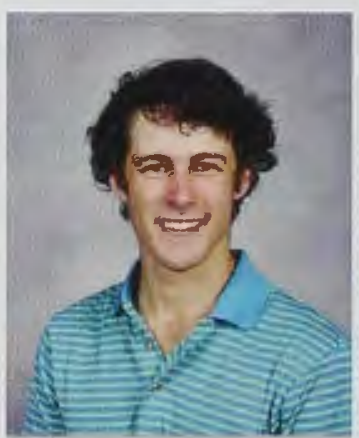

Caleb Curby

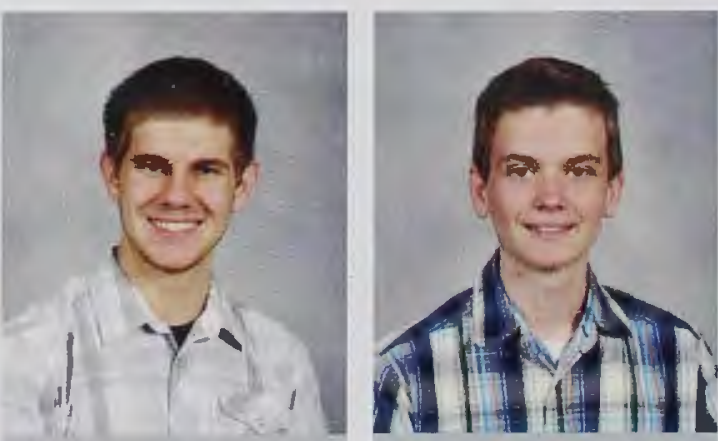

David Elgersma

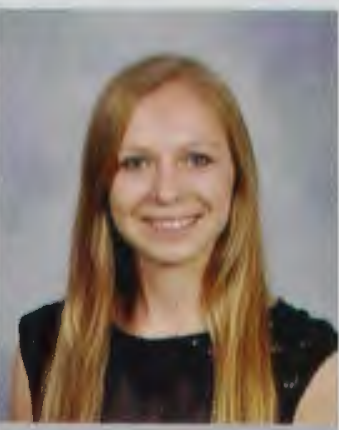

Kayleigh Artz

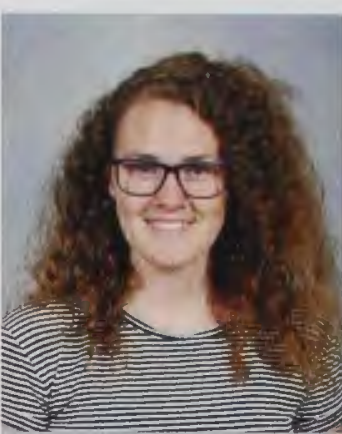

Abbie Belcher

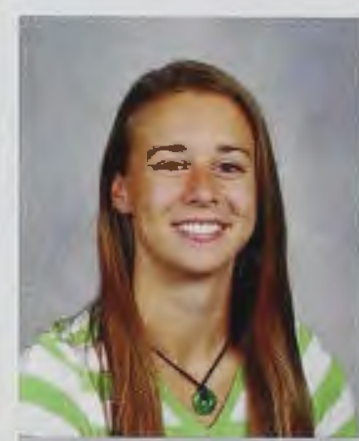

Breanne Brigadoi

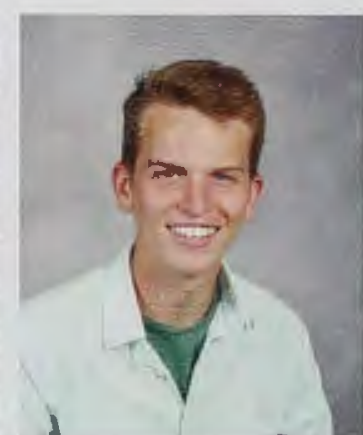

Jesse Childress

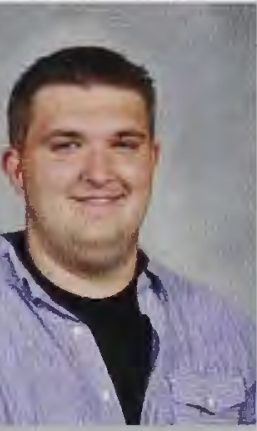

Jonathan Czyzewski

Harrison Elkins

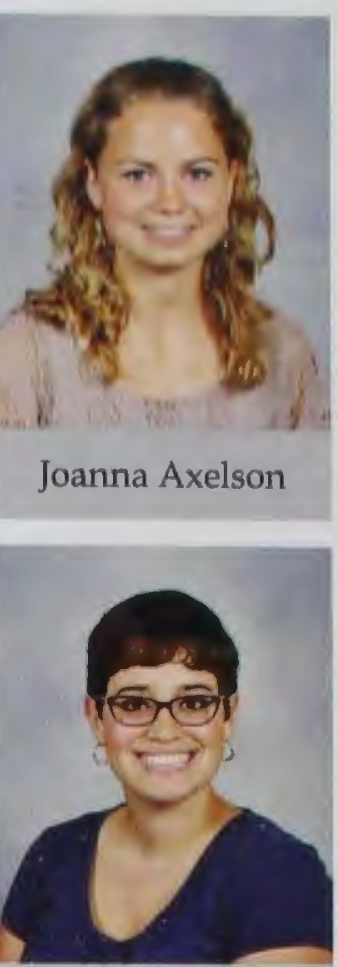

Emilie Belyea

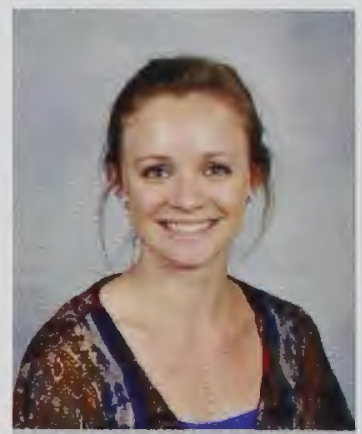

Jillissa Brummel

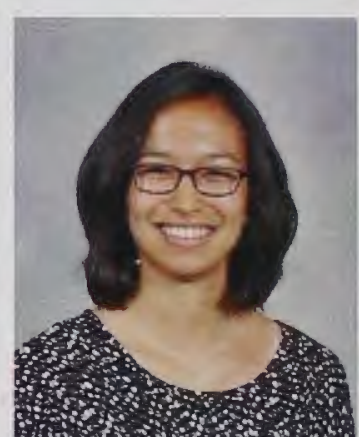

Lindsey Chiu

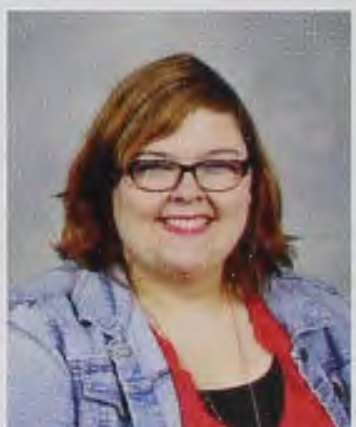

Amanda Davidson

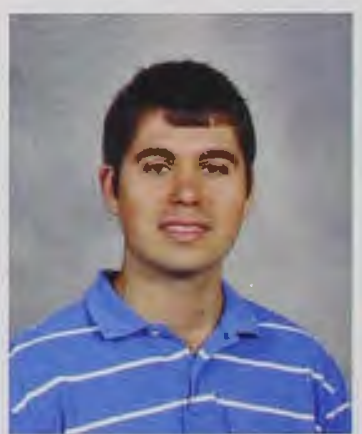

Andrew Ellicott

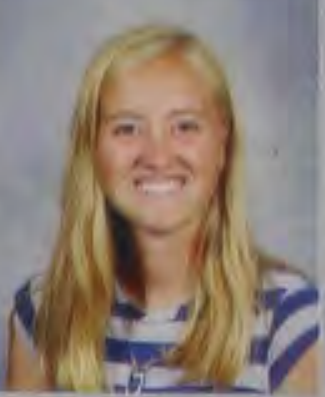

Brooke Baldwin

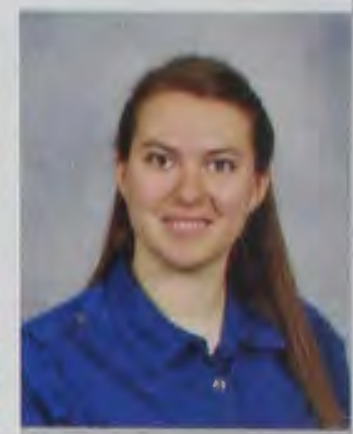

Sara Bennett

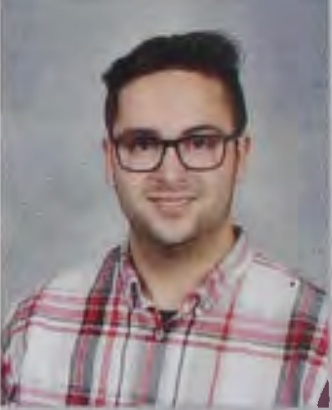

Cameron Busdiecke:

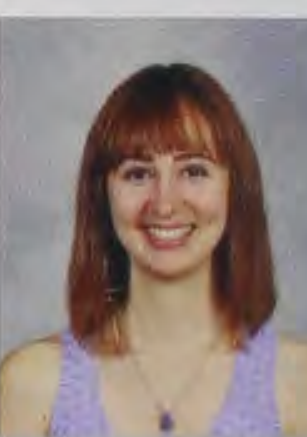

Maria Christini

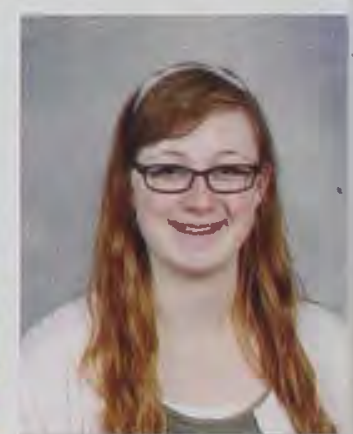

Shannon Davis

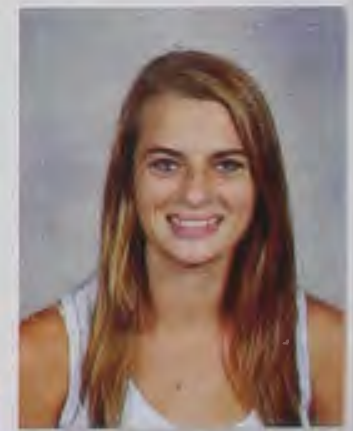

Olivia Esbenshade 


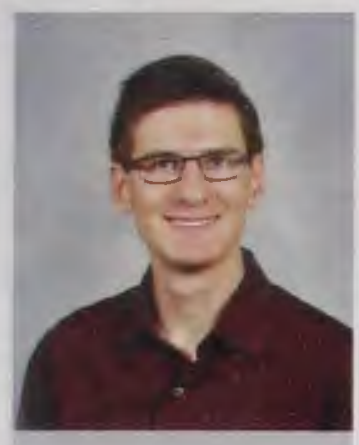

Scott Eshelman

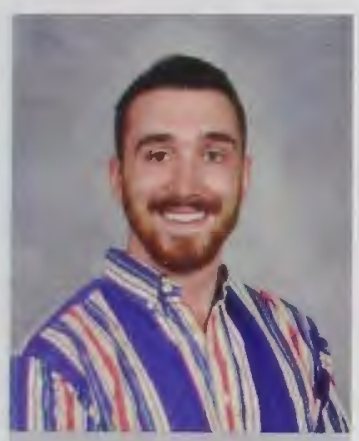

Matthew Grafton

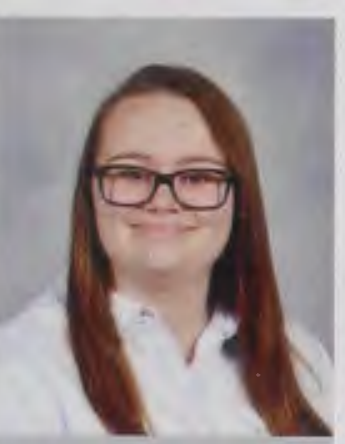

Erin Hayes

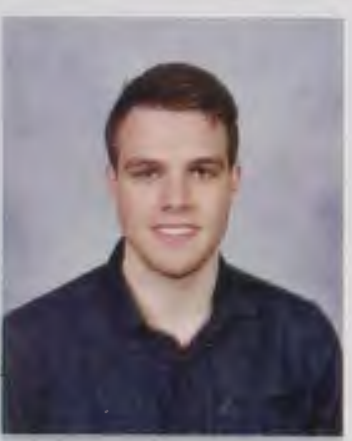

Aaron Hostetler

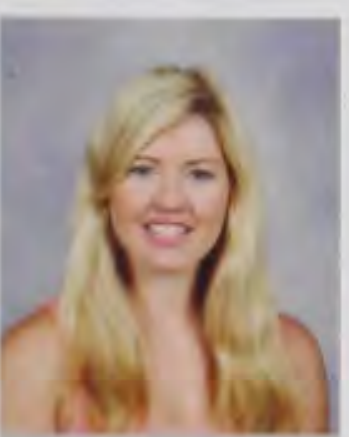

Audra Kearney

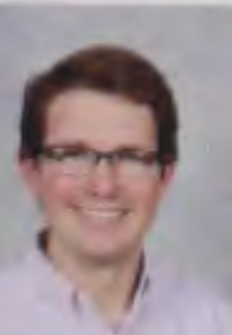

Ionathan I vons

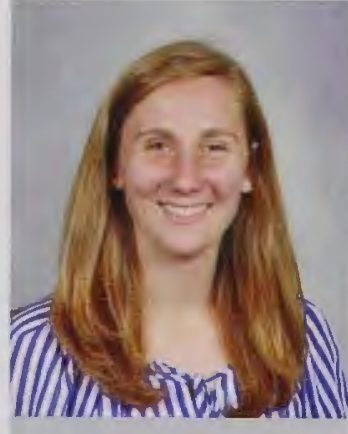

Abigail Fleming

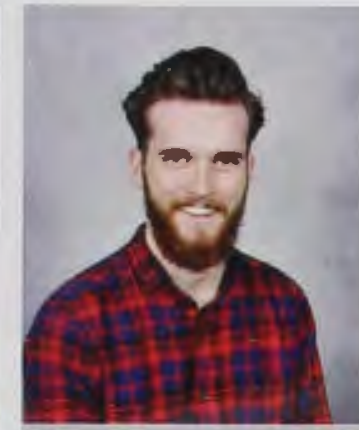

Peyton Green

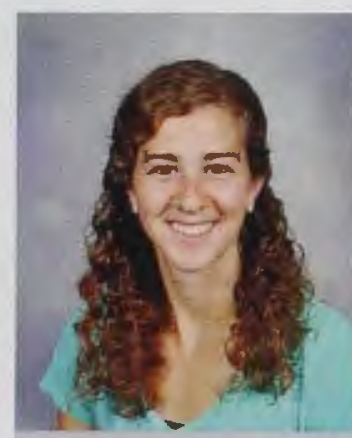

Vivianna Hein

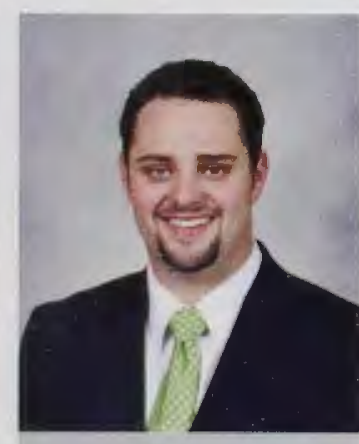

Daniel Hulbert

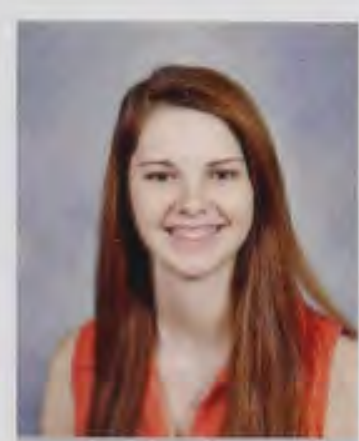

Lauren Kemme

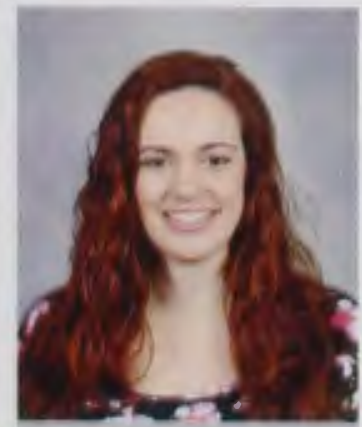

Natalie Macek

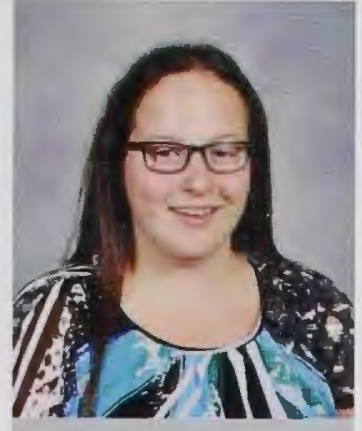

Jacqlyn Fletcher

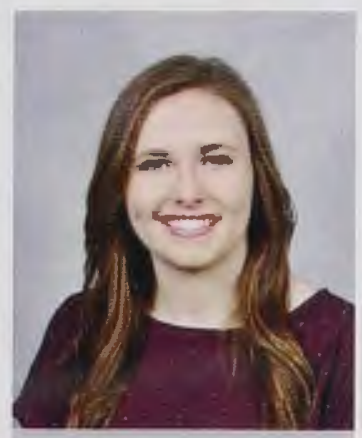

Rebecca Grier

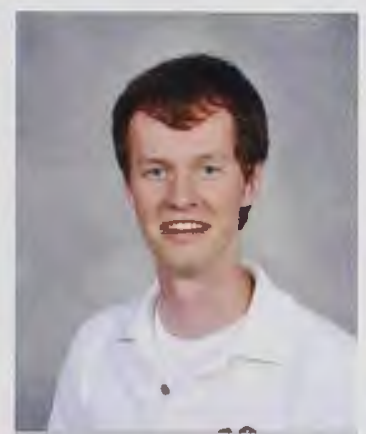

Dillon Henschen

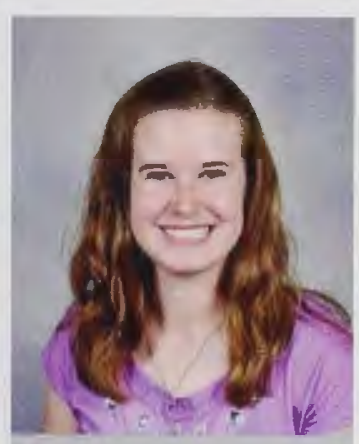

Alison Hunter

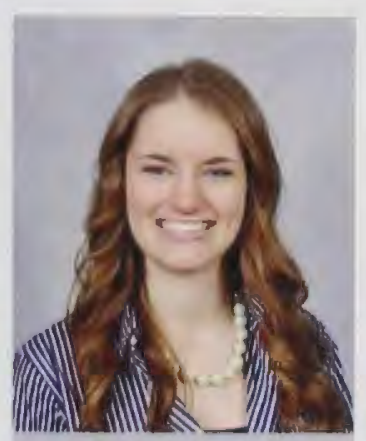

Megan Lawrence

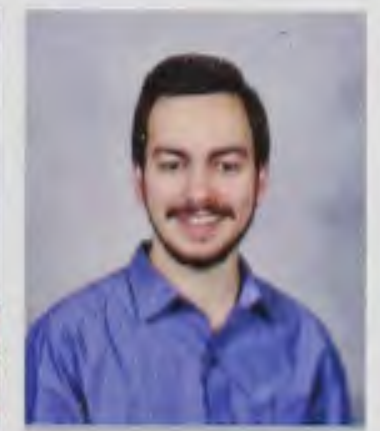

Ryan Marquardt

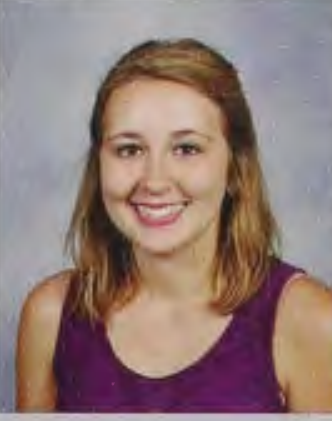

Lindsey Ford

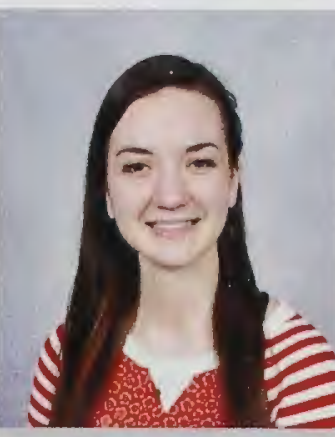

Amy Grudier

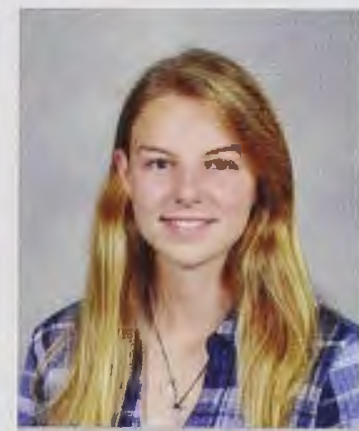

Sarah Hess

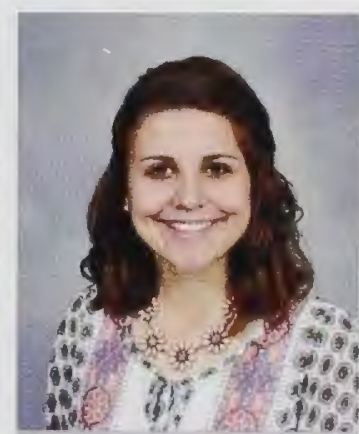

Mallory Jefson

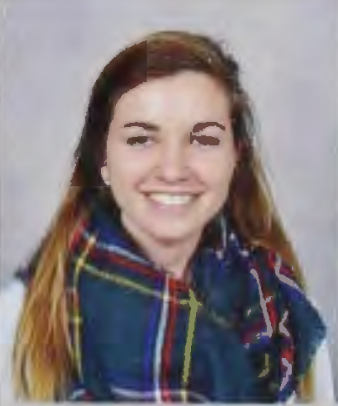

Emma Leppke

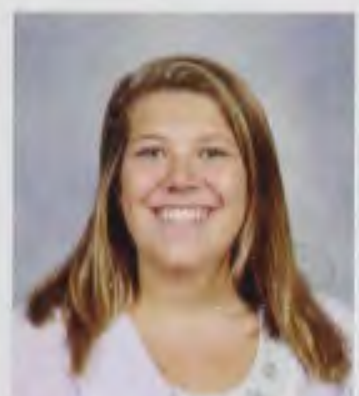

Abbey Martin

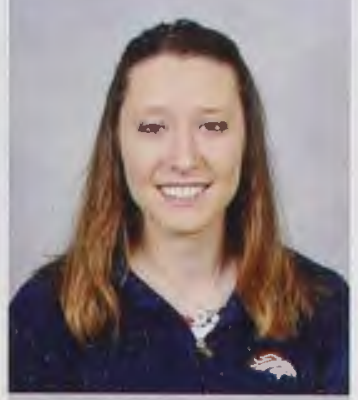

Emily Gentry

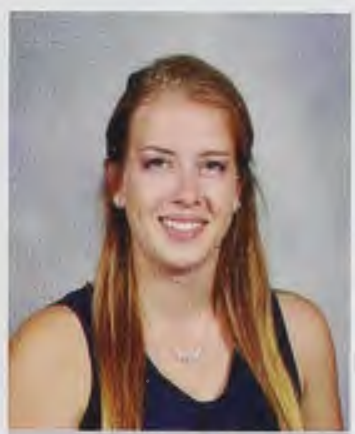

Kasey Hannay

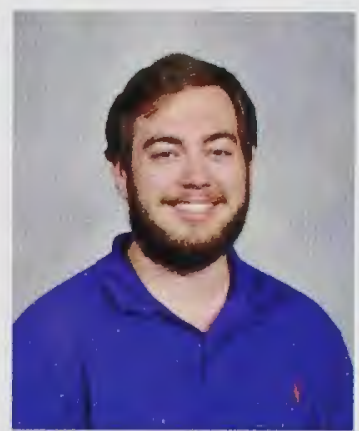

John Hilderbrand

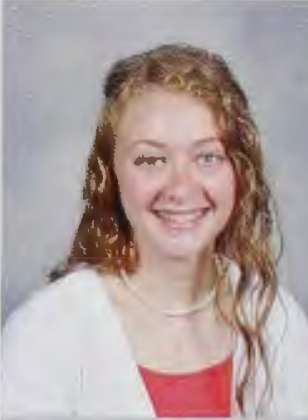

Esther Jones
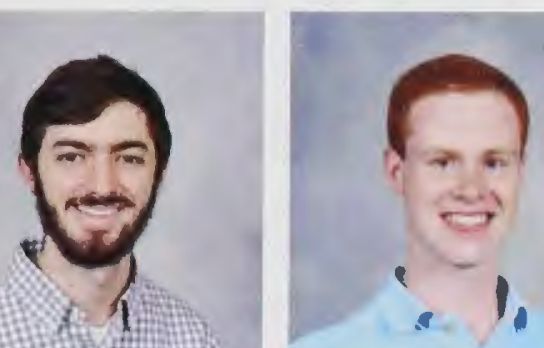

Lynn Long

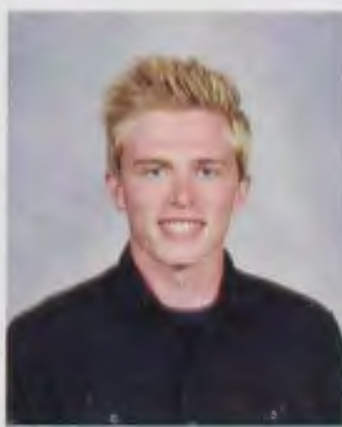

Benjamin McClain

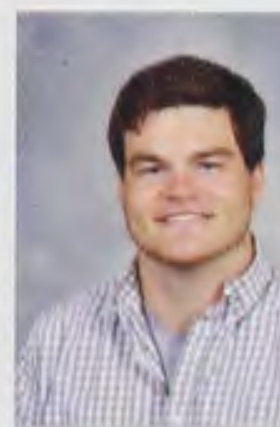

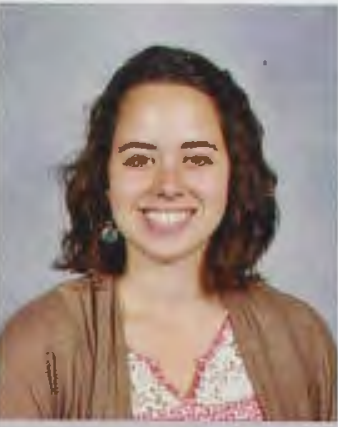

Aubrey Gillette

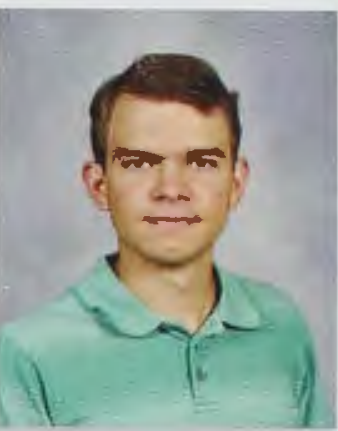

Nathan Harold

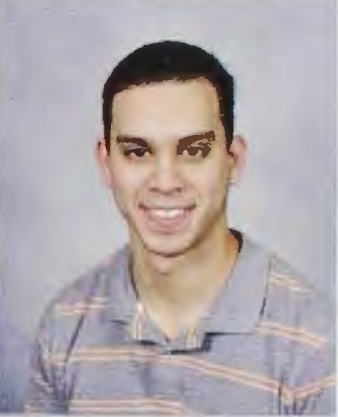

Josiah Hilerio

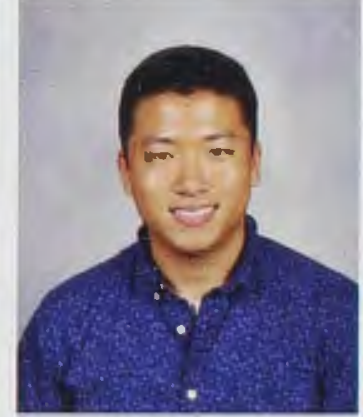

Wesley Kane

Matthew Merical

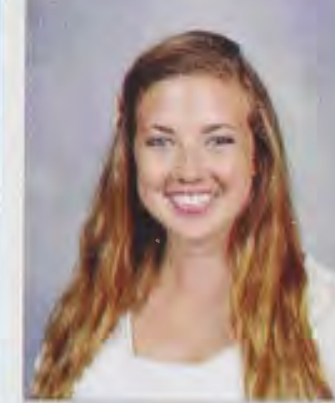

Brooke Lowry

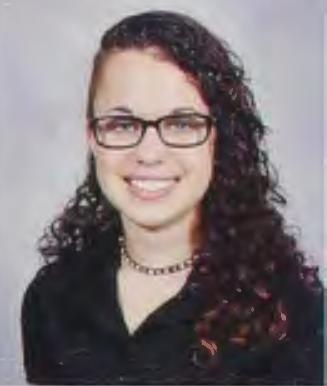

Naomi Harward

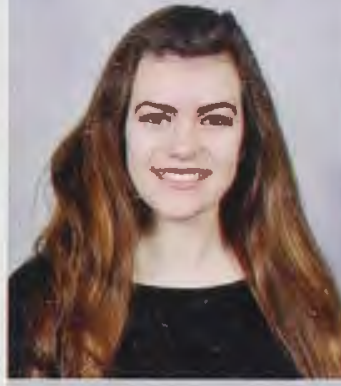

Jessica Holdeman

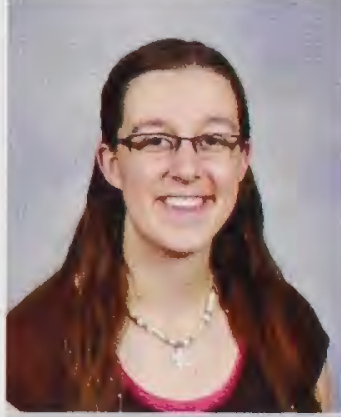

Faith Karg

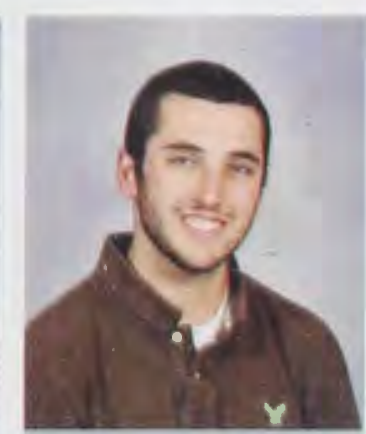

Christian Mickey 


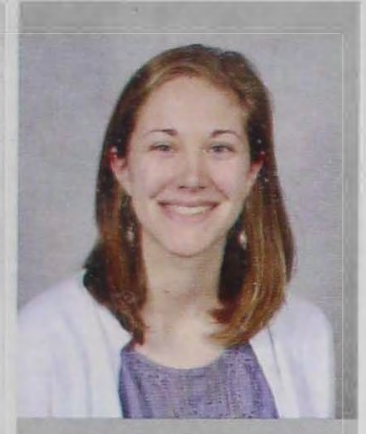

Sarah Taylor

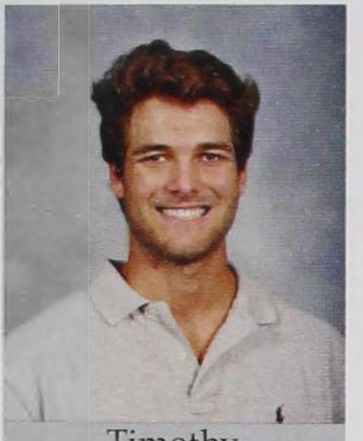

Timothy Vanwingerden

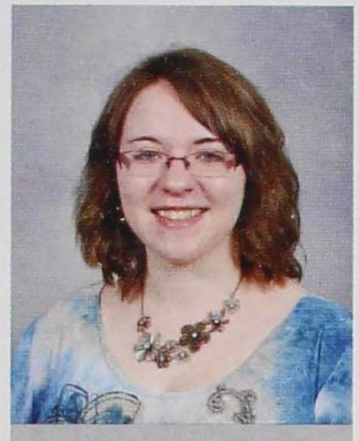

Michaela Wade

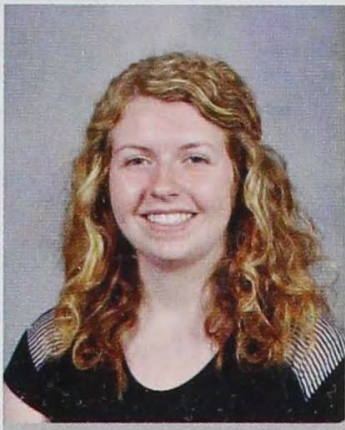

Jessica Wall

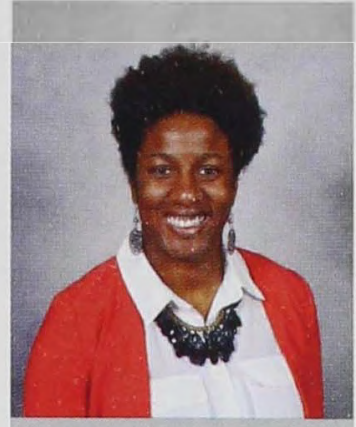

Jervonne Ward

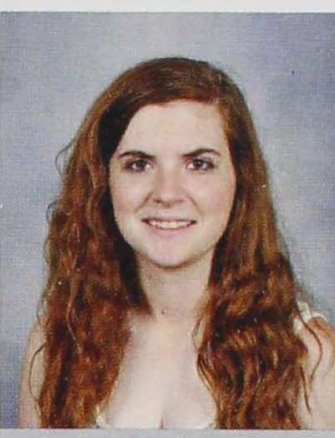

Kelcie Wellendorf

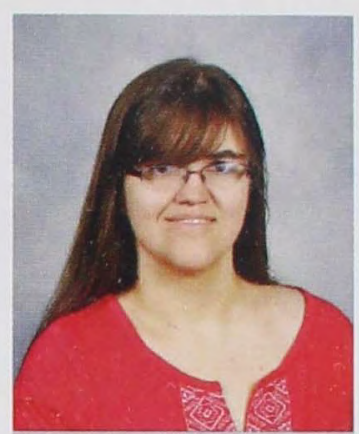

Alathea Westbrook 


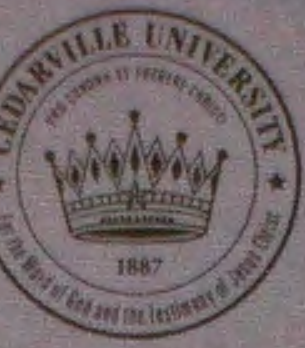

The 120th Annual

COMMENCEMENT

Cedarville University

CLASS of 2016

Saturdey, the Seventh Day of May

Two Thousand and Sixteen

at Ten Q'clock in the Morning

Dodan Field House

Cadanilte University

Cedarville, Ohio

GENERAL ADMISSION

Adrot One

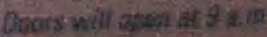

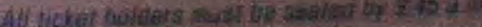





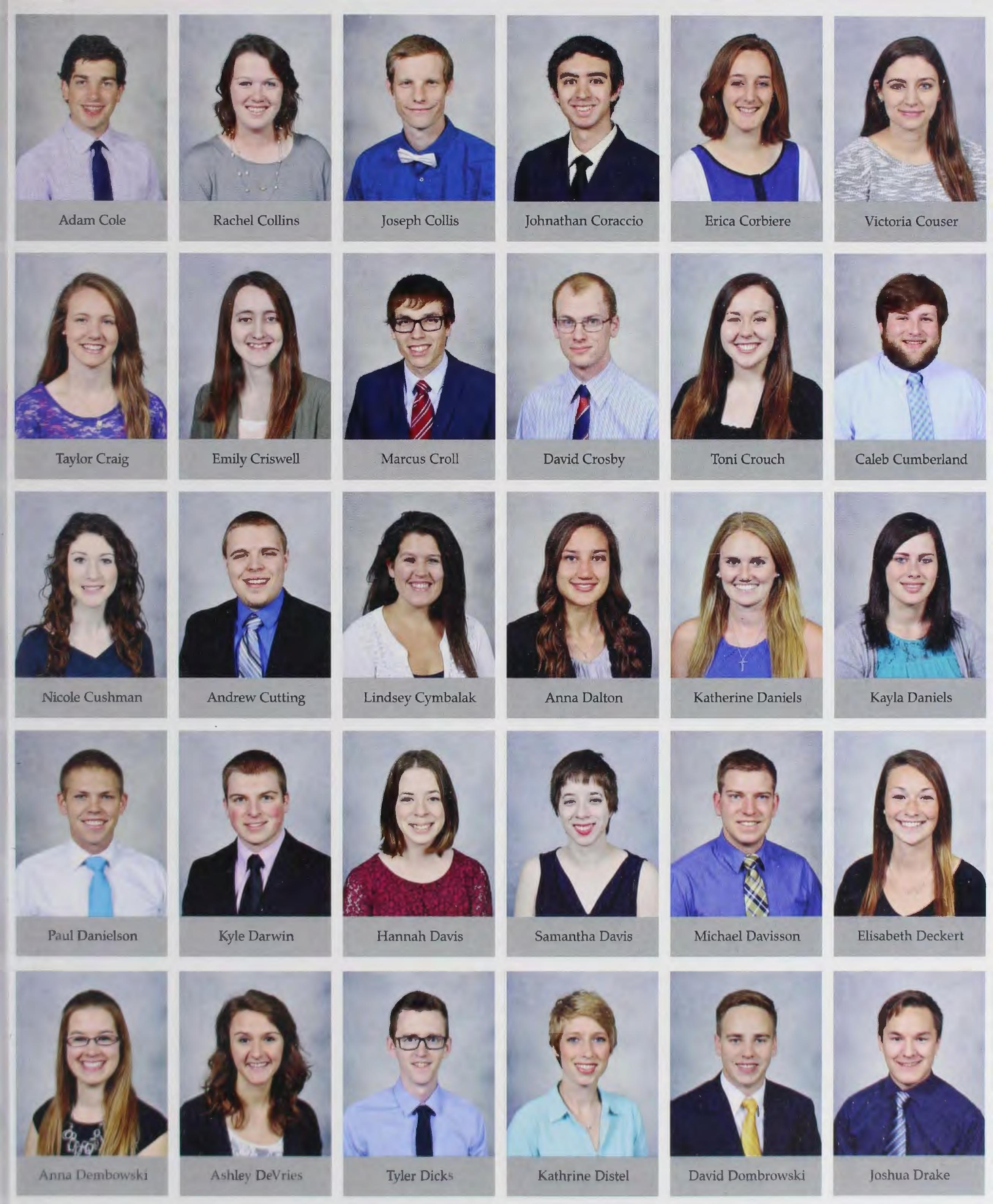




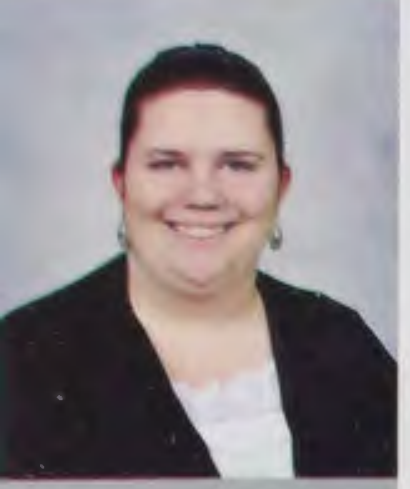

Meghan Duff

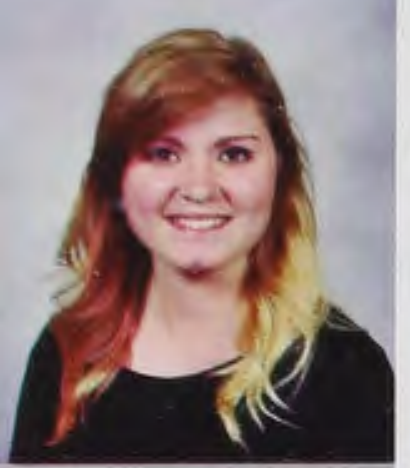

Carissa Engle

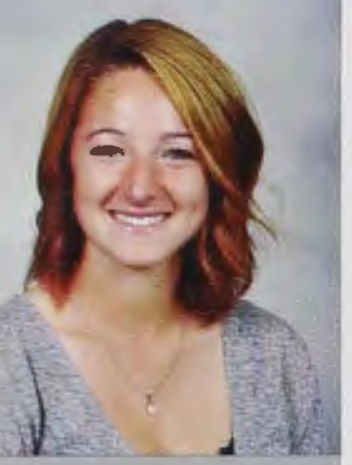

Kylie Faxon

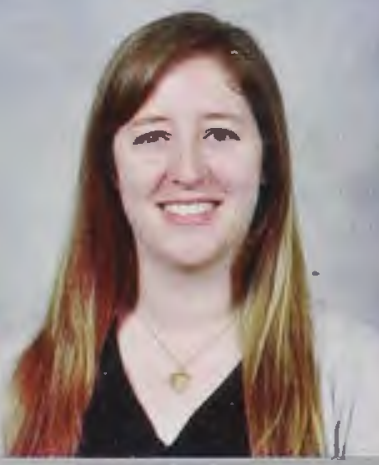

Sarah Fox

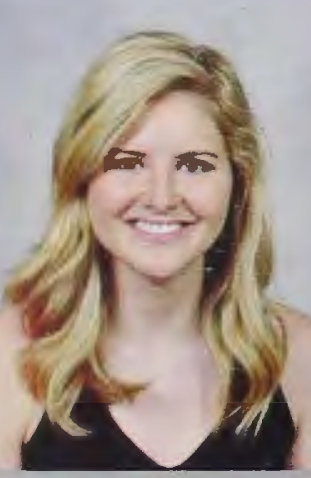

Shannah Gardiner

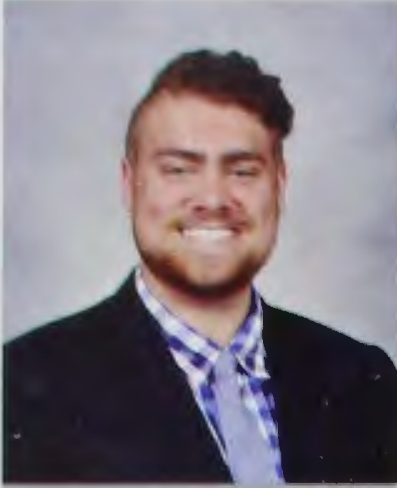

William Duncan

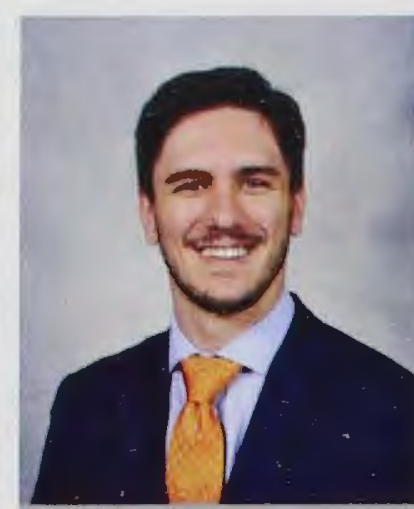

Grant English

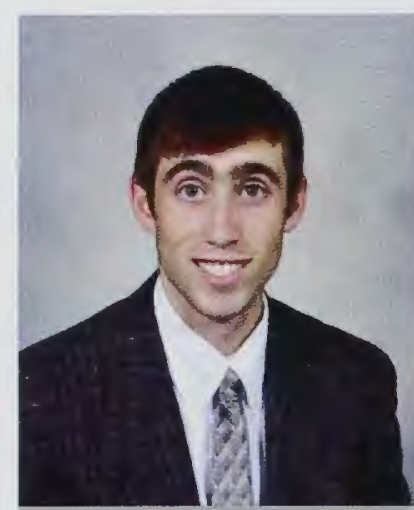

Luke Febrizio

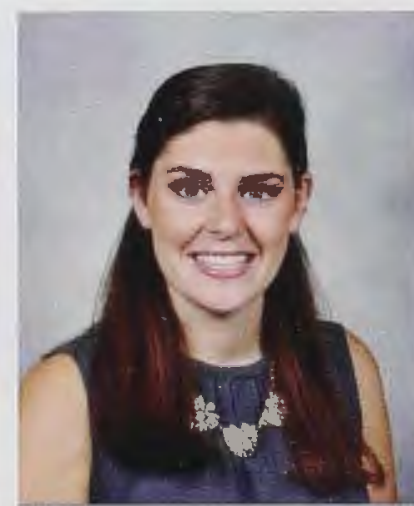

Ellysa Frey

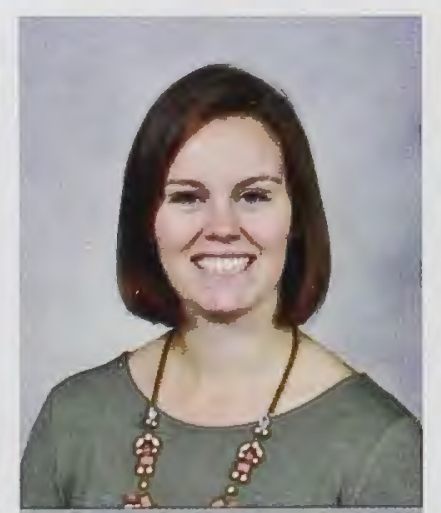

Hannah Gendron

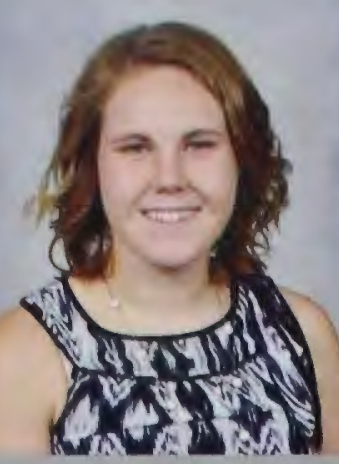

Hannah Eckberg

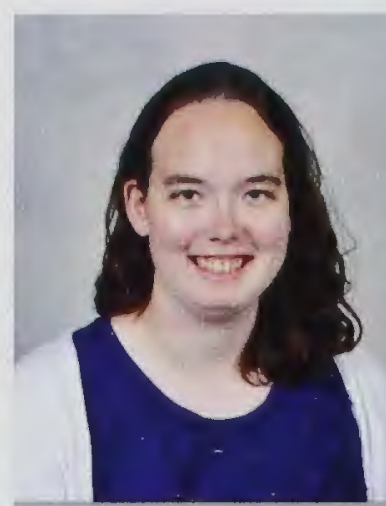

Michelle Eriksen

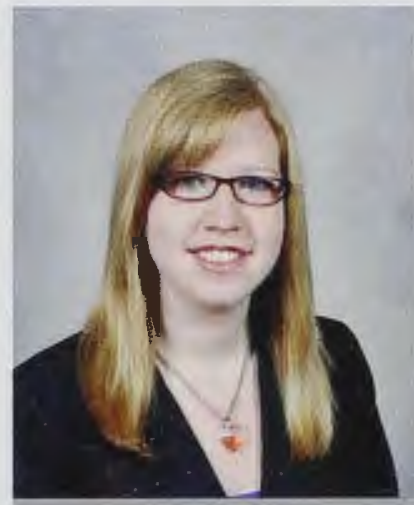

Kathryn Field

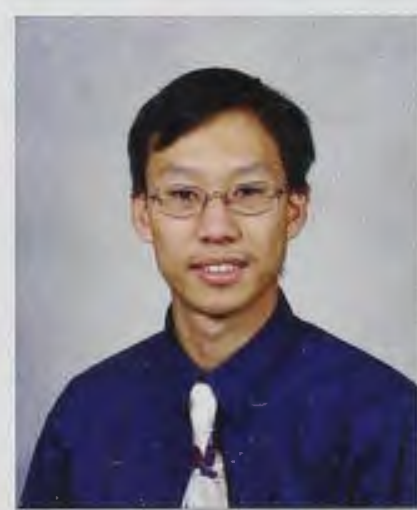

Ezra Fu

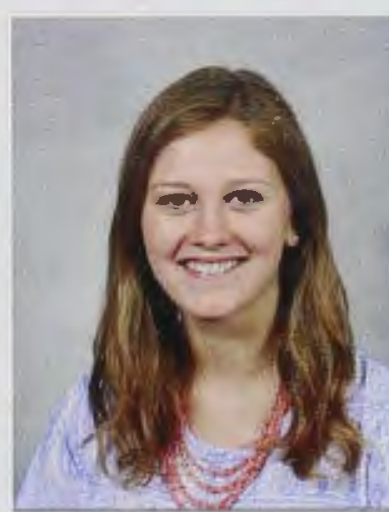

Rebecca Gendron

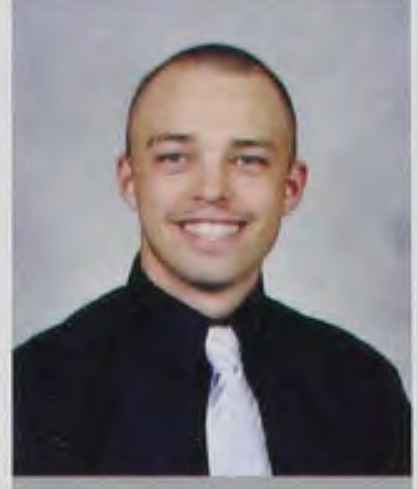

Justin Eckberg

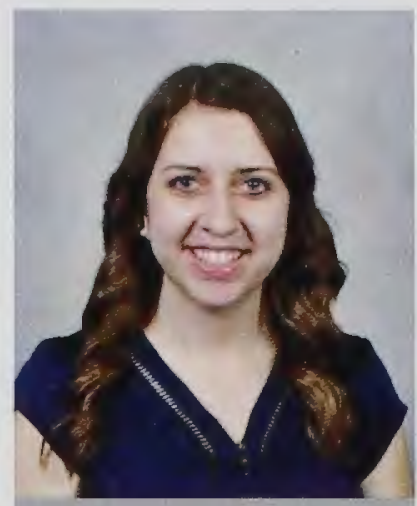

Jessica Evanoff

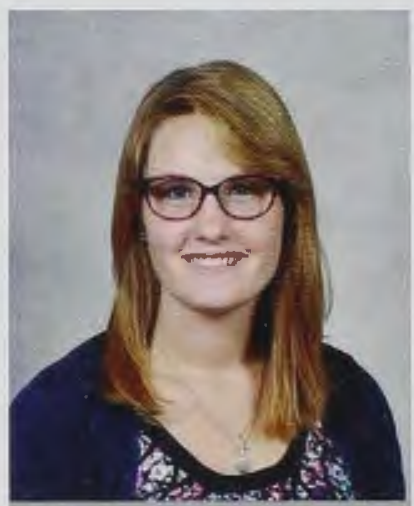

Caitlin Fillmore

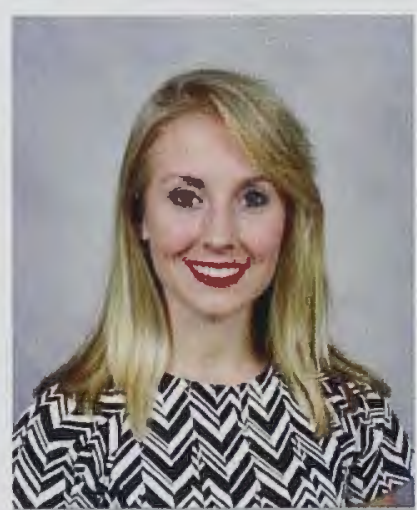

Holly Fudge

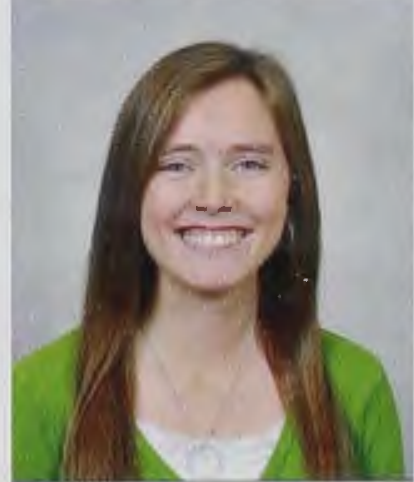

Kelsey Gentry

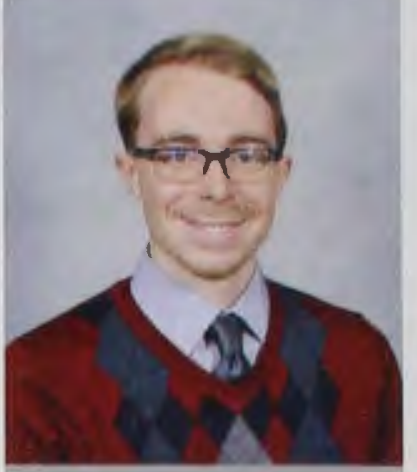

Andrew Eckley

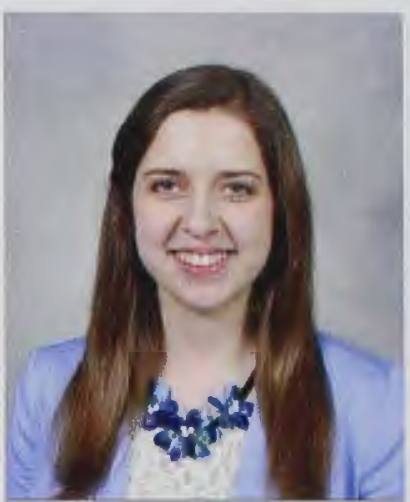

Julia Evanoff

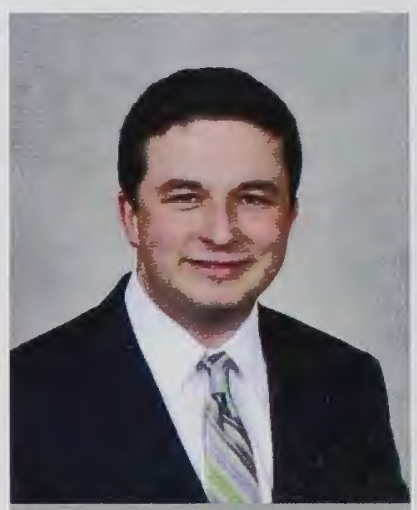

Logan Fitzgerald

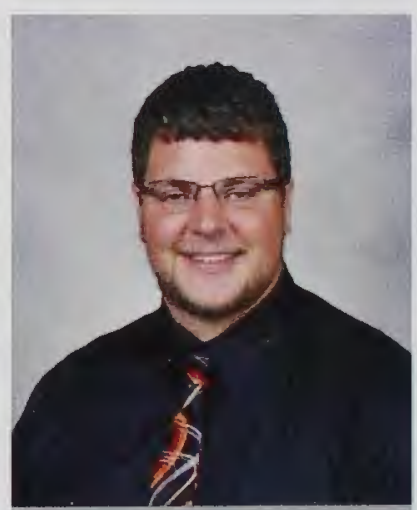

Brian Funk

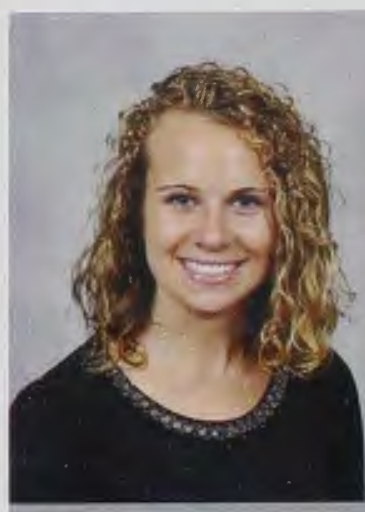

Jordan Gilbert

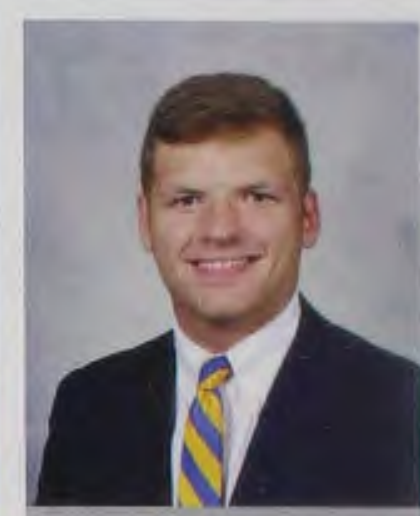

Benjamin Fawcett

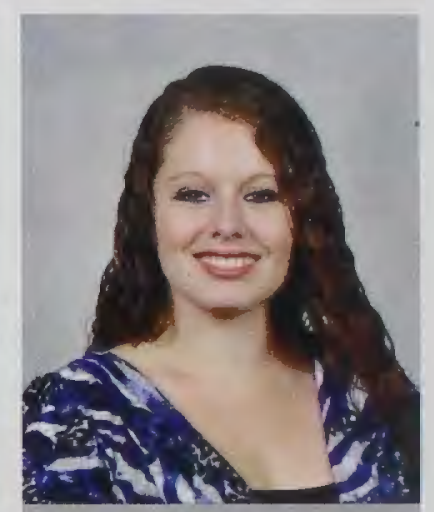

Kaitlyn Fox

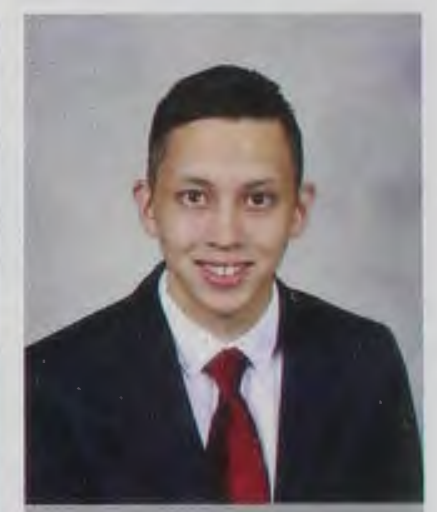

Jonathan Gallardo

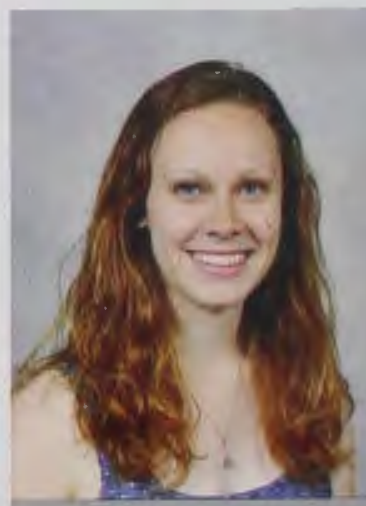

Brenna Gilson 


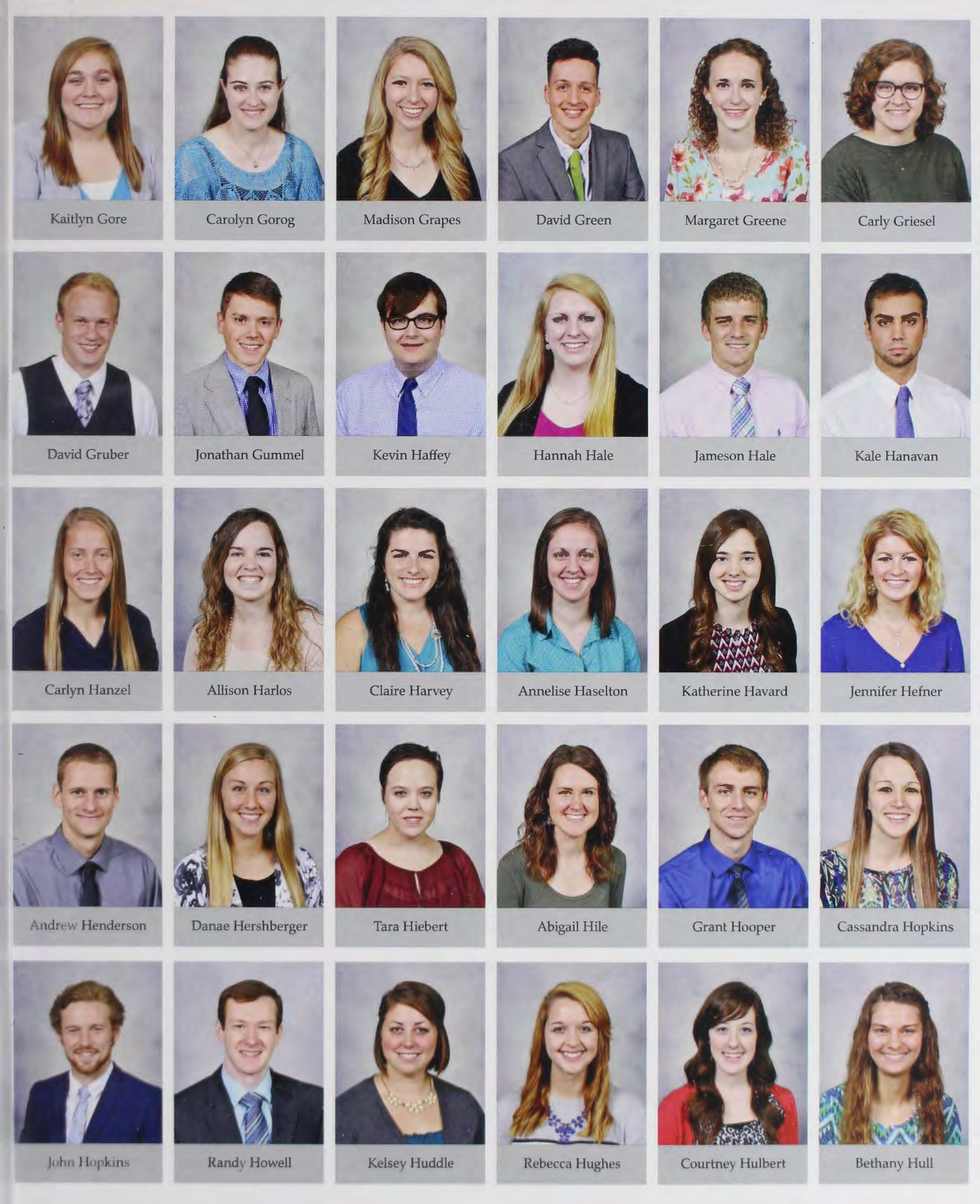




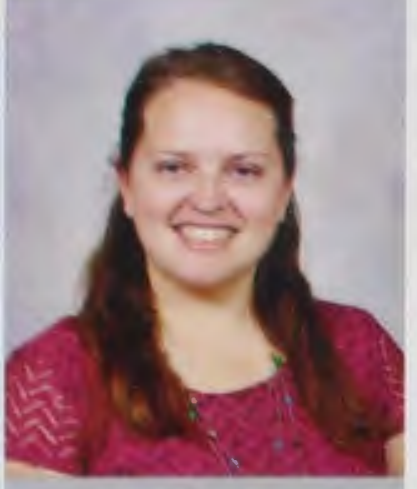

Danielle Hutchison

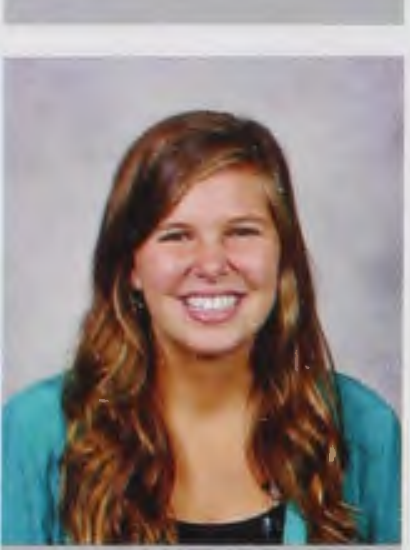

Alicia James

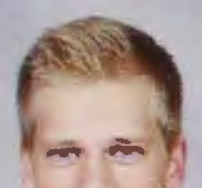

$\stackrel{2}{\longrightarrow}$

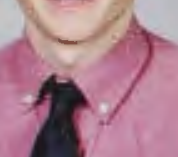

Steven Kauffmann

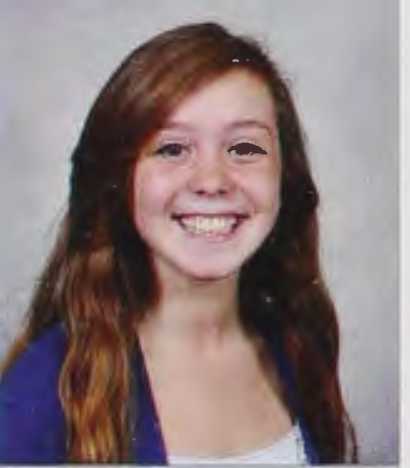

Angelica Kester

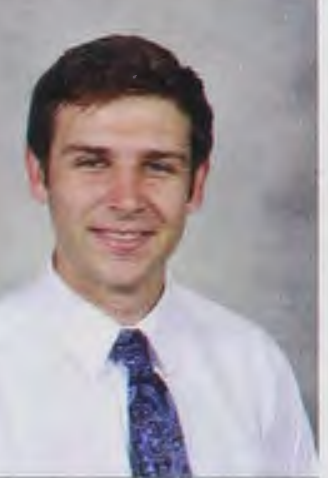

Jared Krater

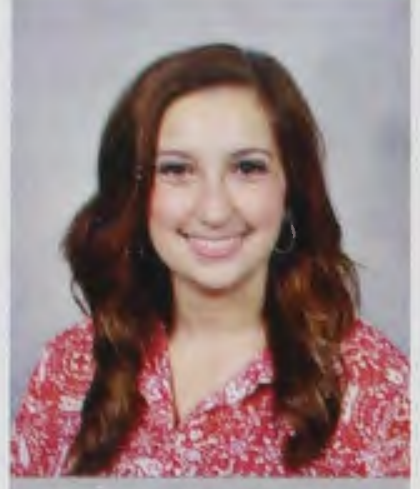

Jennifer Hutchison

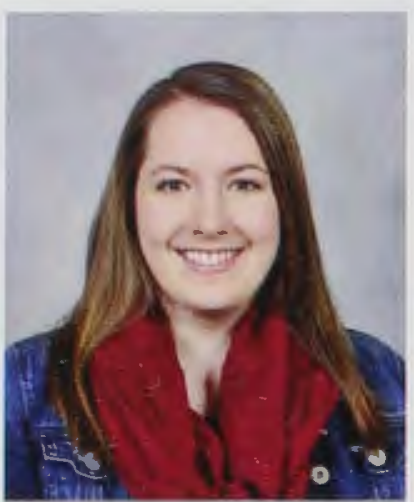

Zoe Jamison

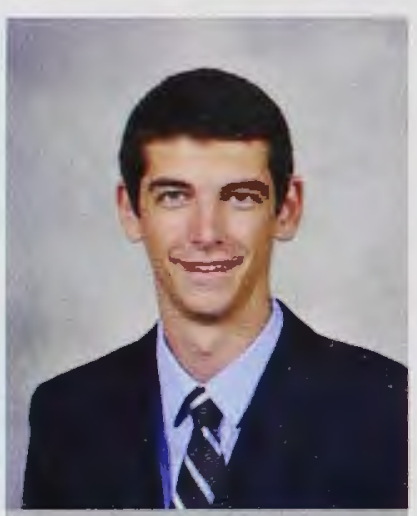

William Keating

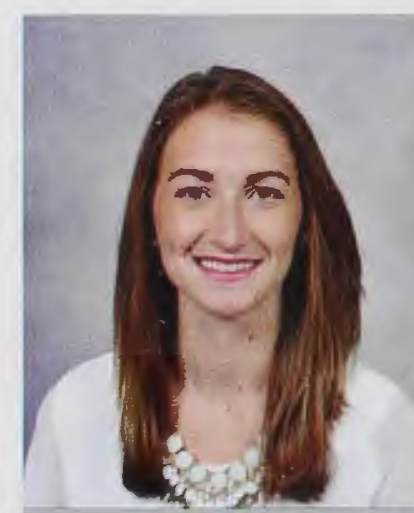

Kathleen Kidd

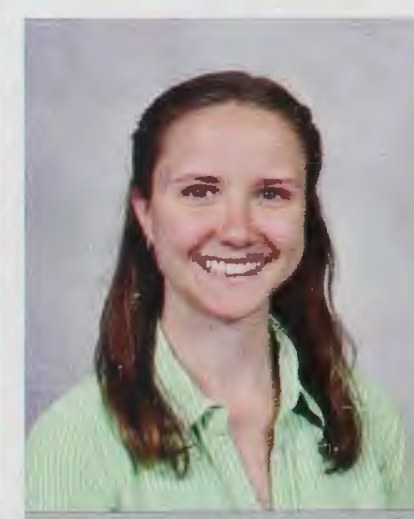

Laura Krizo

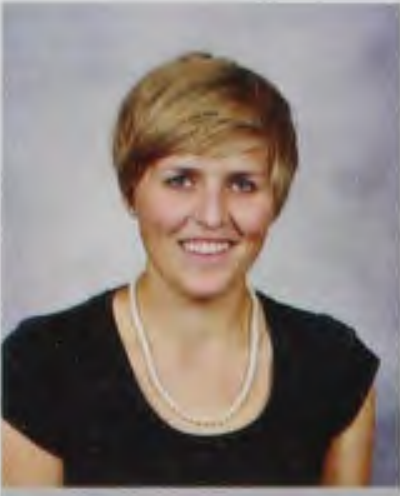

Stephanie Huyser

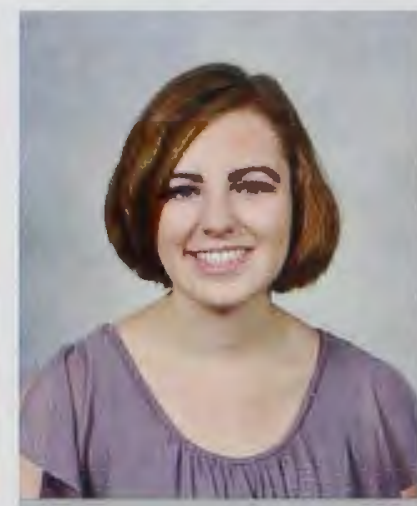

Allison Jensen

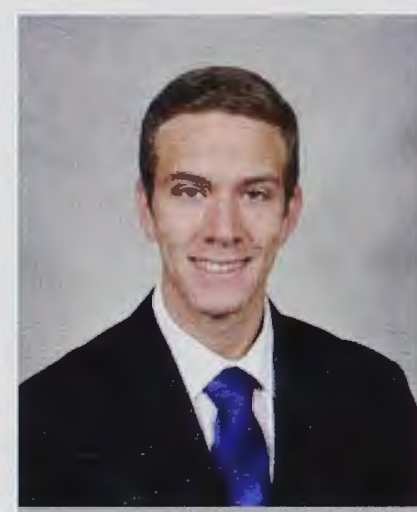

Richard Kelly

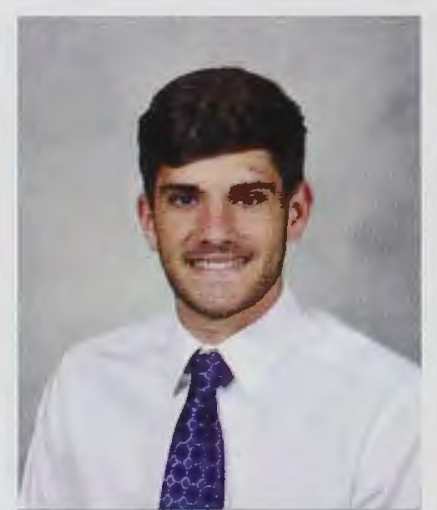

Nathan Kilgore

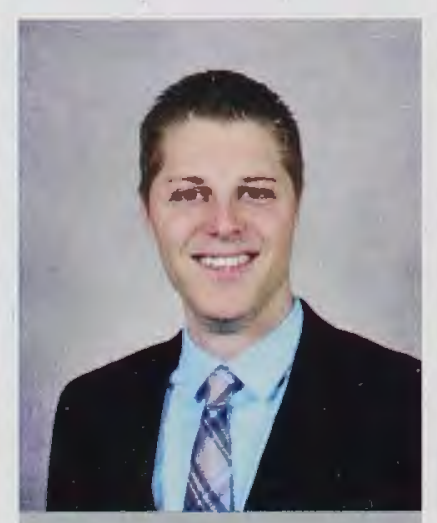

Collin Kueter

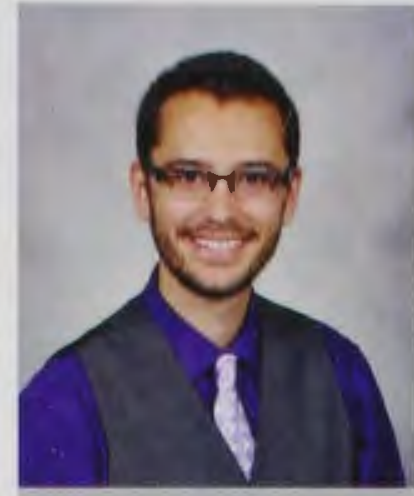

Benjamin Ingis

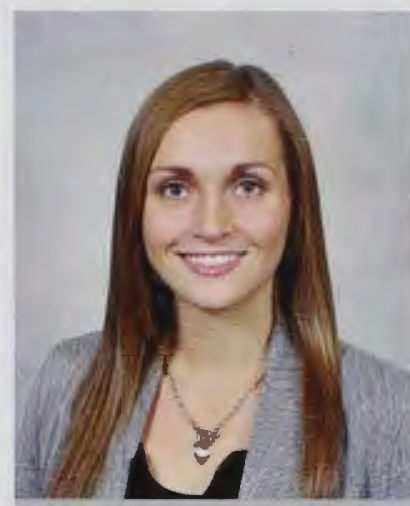

Christa Johnson

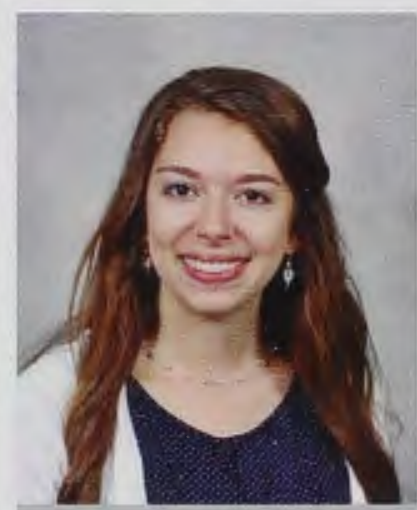

Kaitlyn Kenniv

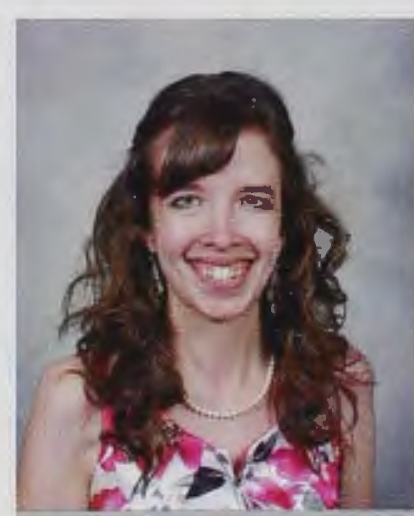

Michelle Kisha

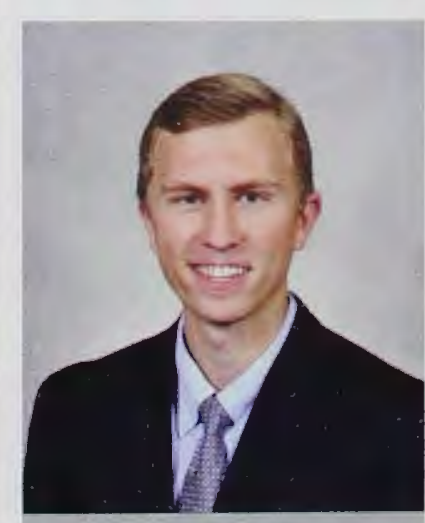

Michael Kuhn

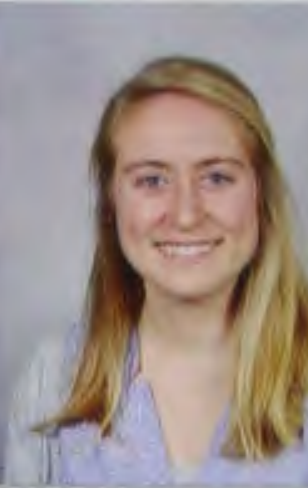

Hannah Ingram

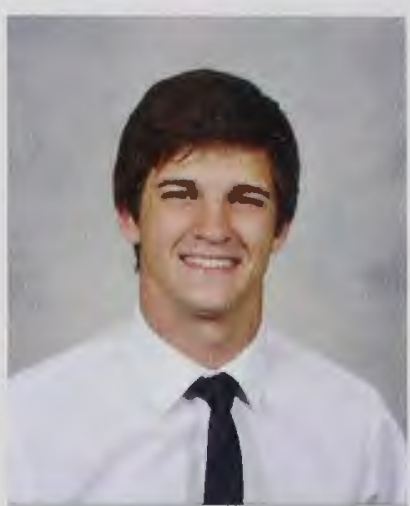

Ryan Johnston

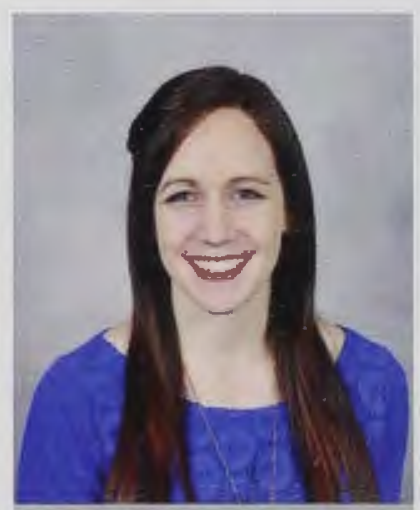

Ellery Kent

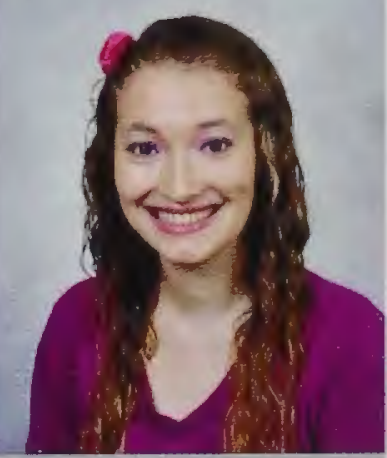

Ayana Kleckner

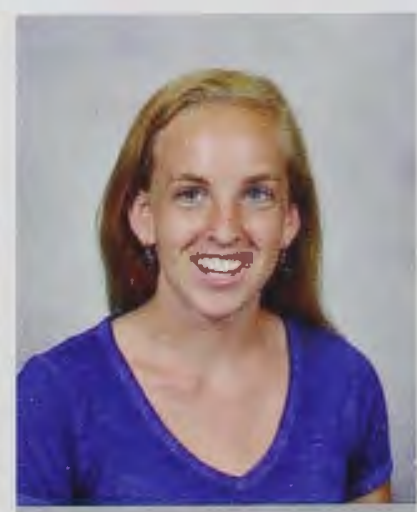

Christine Kuiken

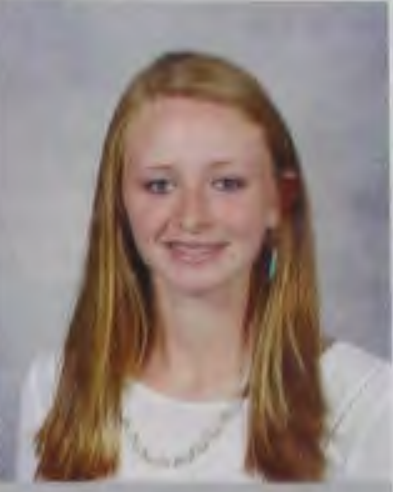

Micaila Susanna Iversen

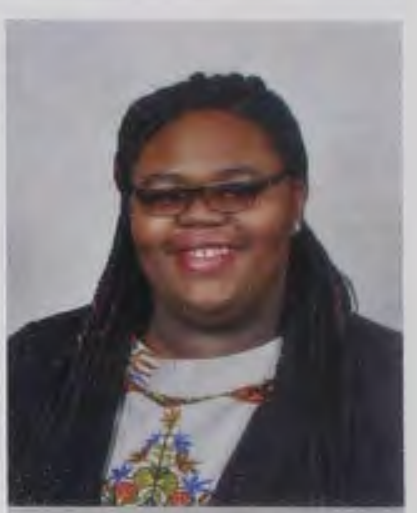

Brittany Jones

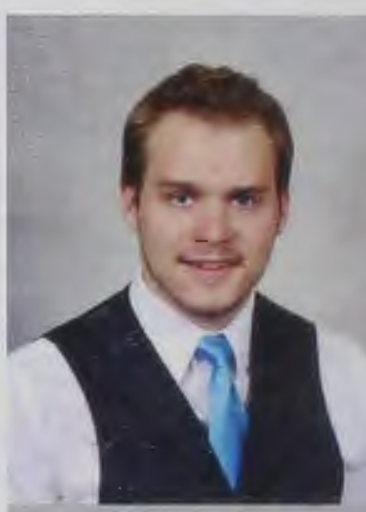

Timothy Kerr

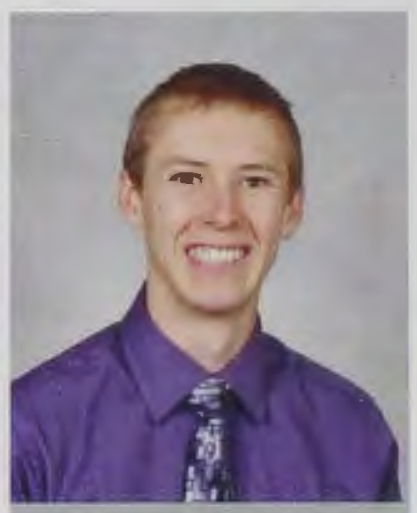

Daniel Kohl

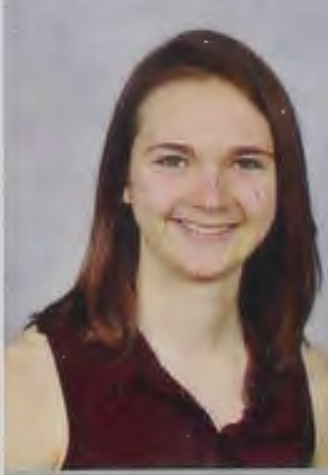

Kristen Laaman 


$$
\begin{aligned}
& \text { EAR日i } \\
& \text { ARARBA } \\
& \text { RABRAR } \\
& \text { A } a=8 \text { ? } \\
& \text { ARRRR }
\end{aligned}
$$




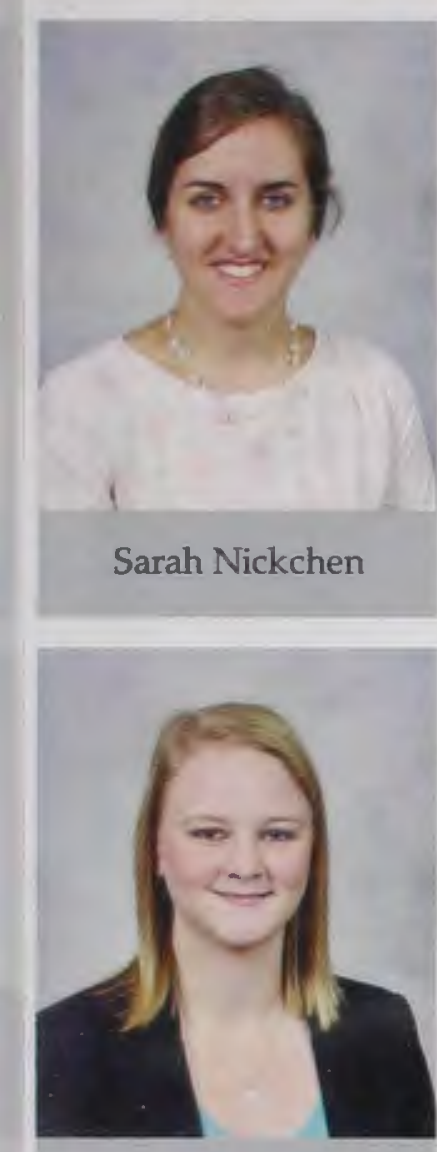

Sarah Orr

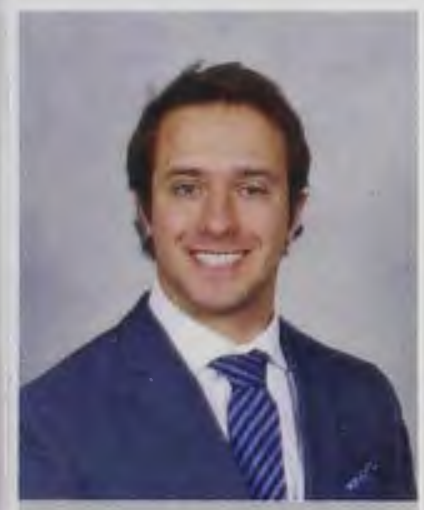

Mitchell Petek

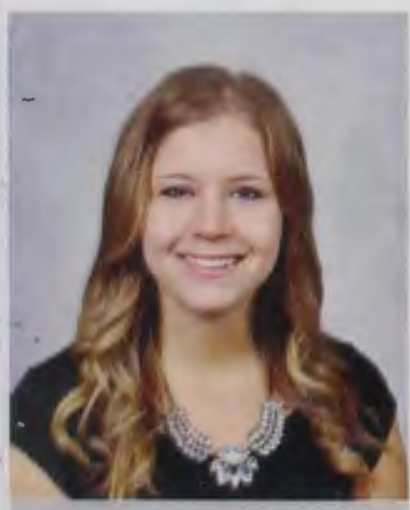

Laura Polsean

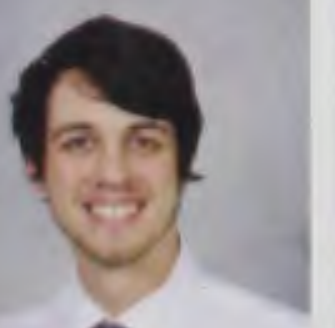

8

Timothy Powers

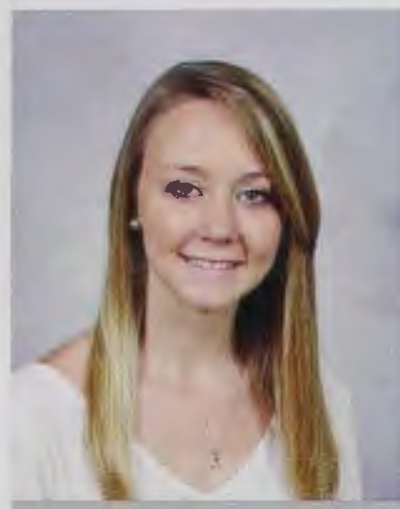

Brenna O'Brien

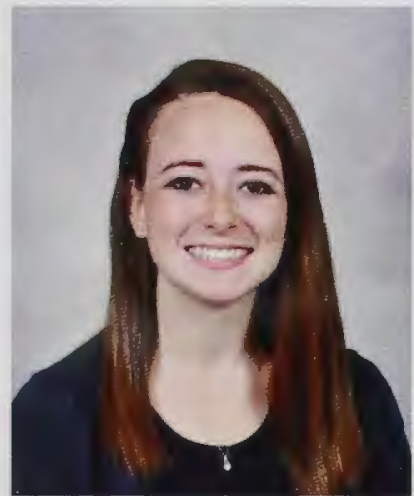

Randi Padilla

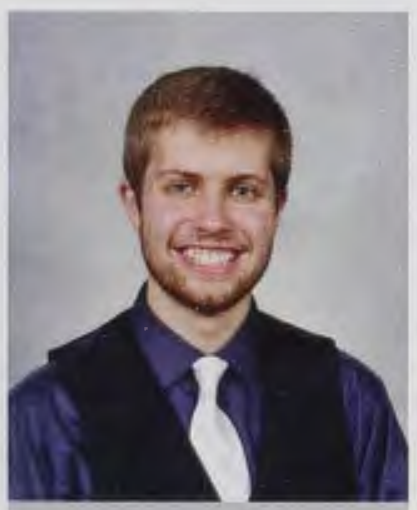

Caleb Peterson

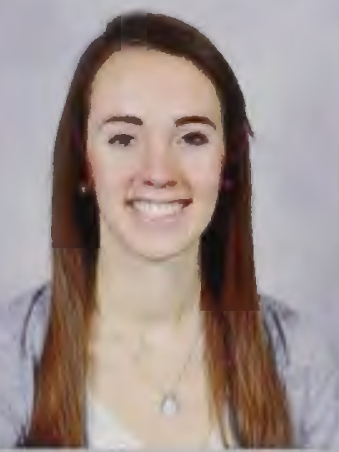

Alyssa Poole

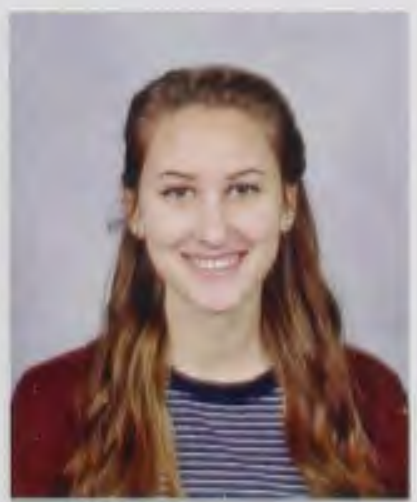

Jenna Pretty

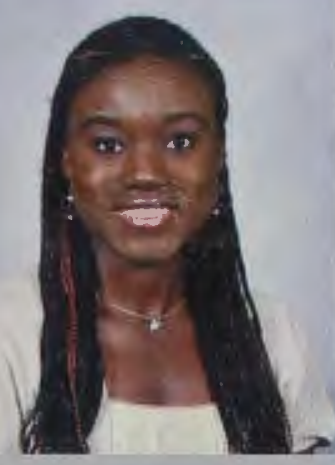

Deborah Obielodan

Sean O'Donnell

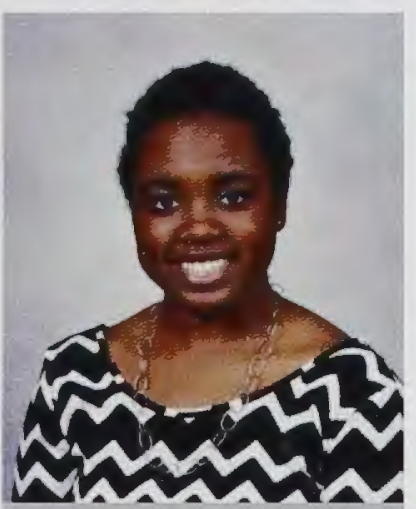

Maya Palmisciano

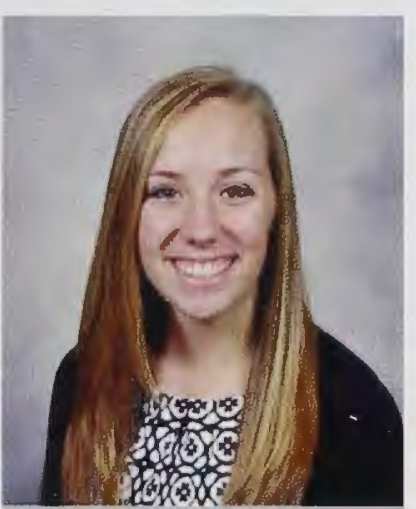

Lindsey Pfeifer

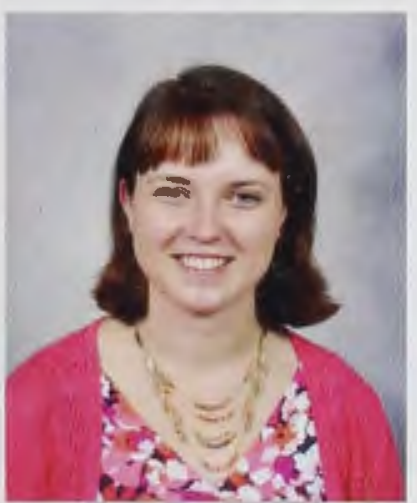

Elizabeth Poore

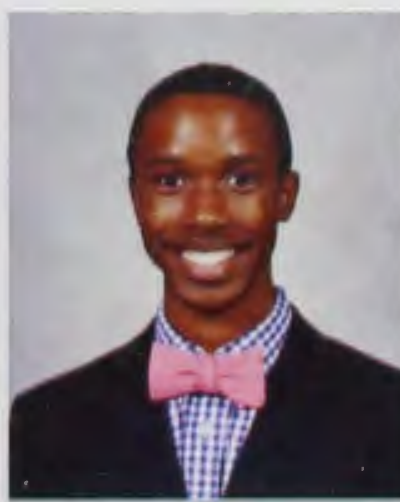

Micah Price

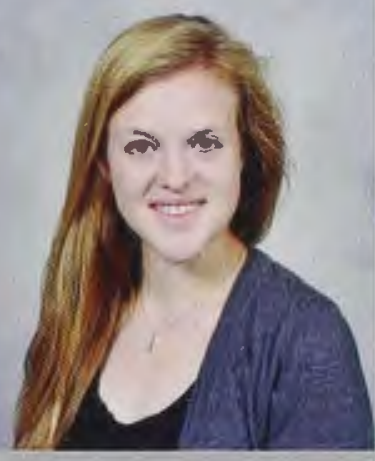

Elise Parsons

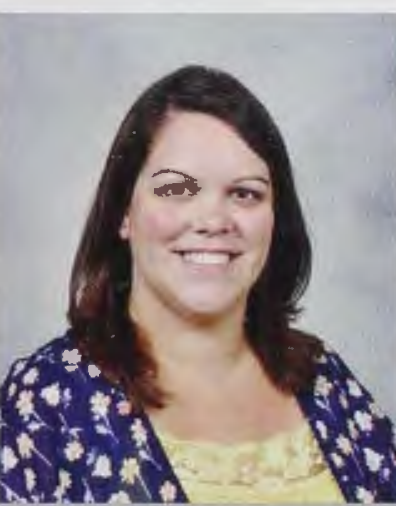

Dawn Phillips

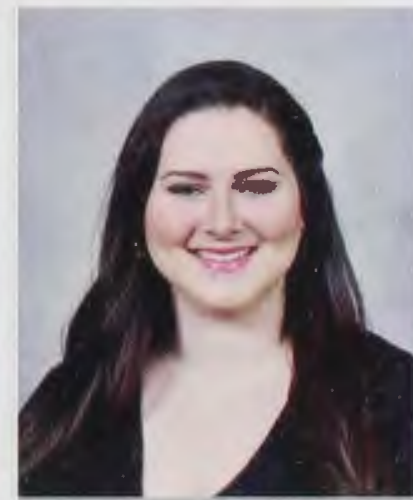

Anna Porter

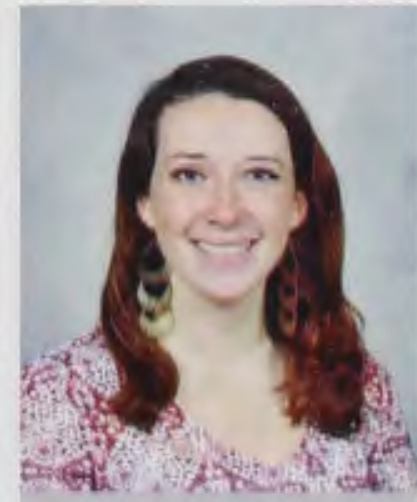

Kelley Pugh

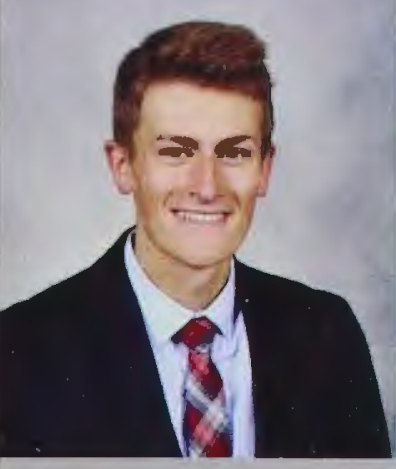

Jacob Olejarczyk

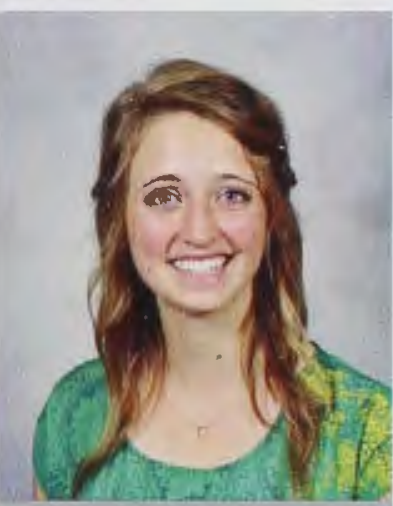

Jennifer Payne

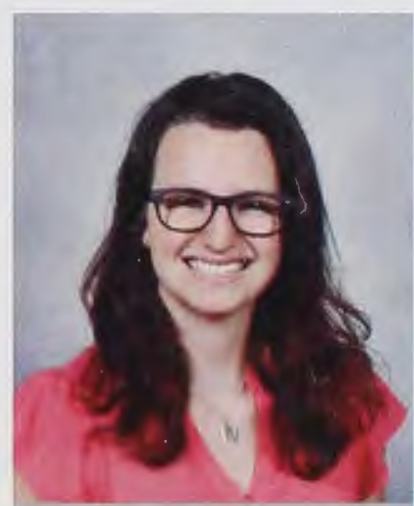

Brielle Pitney

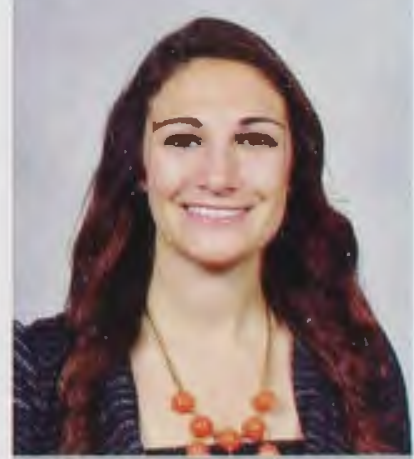

Sarah Powazk

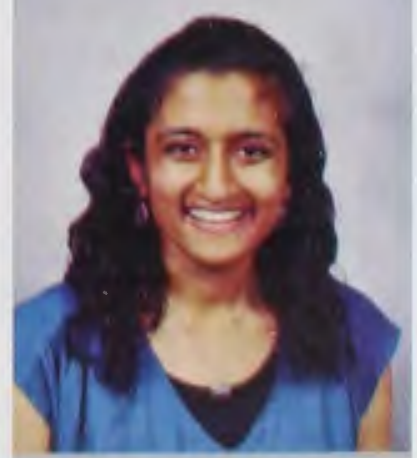

Vineeta Rao

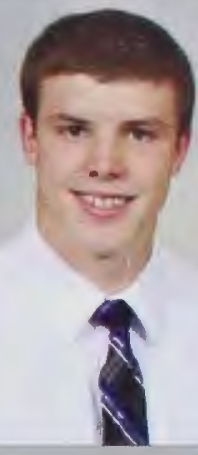

Jesse Orndoff

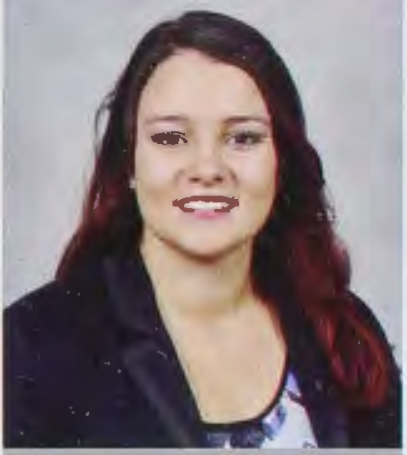

Audree Perry

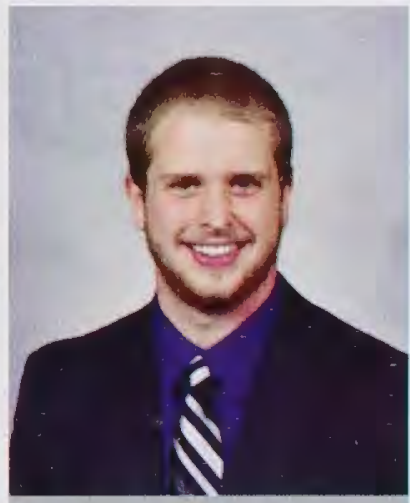

Hunter Poindexter

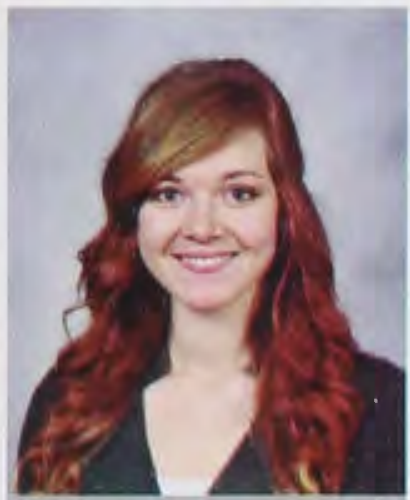

Rijah Powers

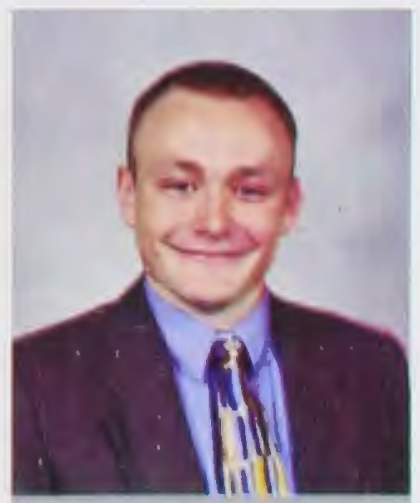

Nathaniel Raquet 


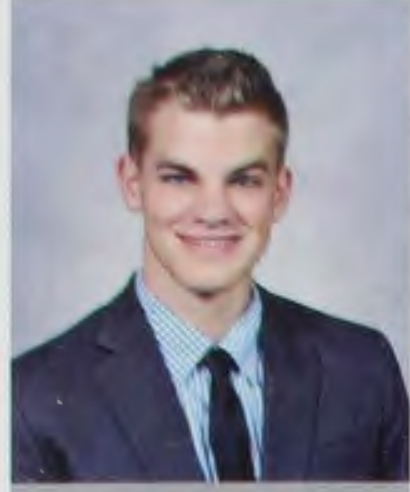

Garrison Reeves

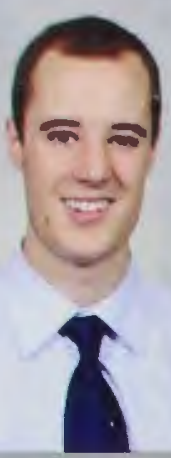

Erik Riggleman

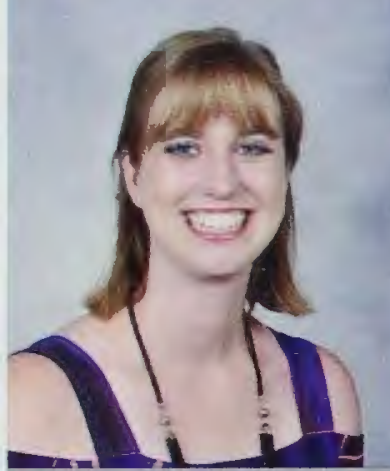

Michaela Ruhlmann

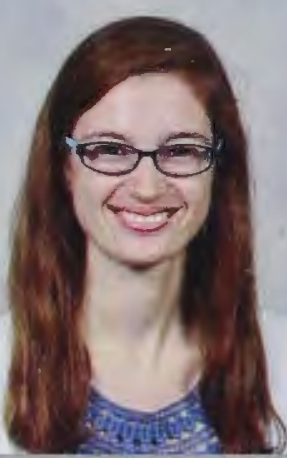

Rachel Schloneger

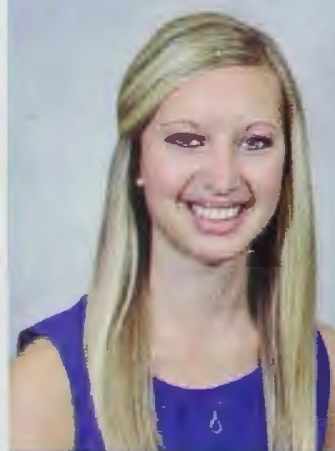

Kara Schutt

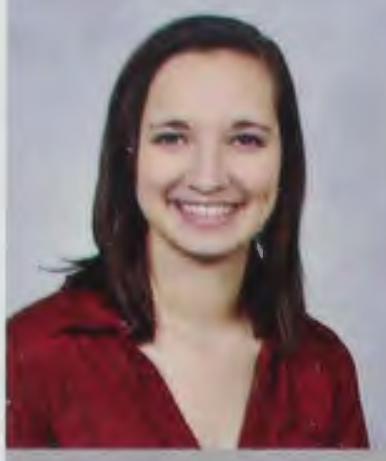

Kristen Reeves

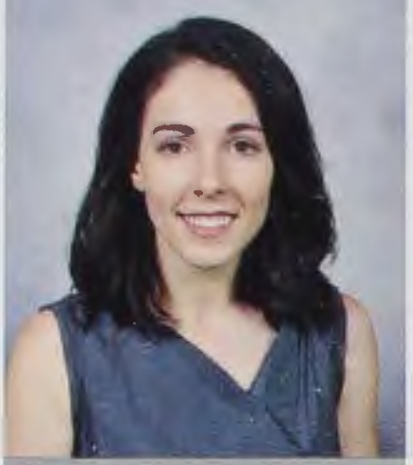

Jocelyn Rivera

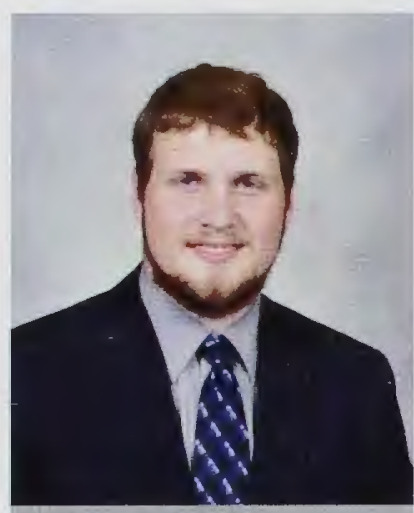

Justin Rumbarger

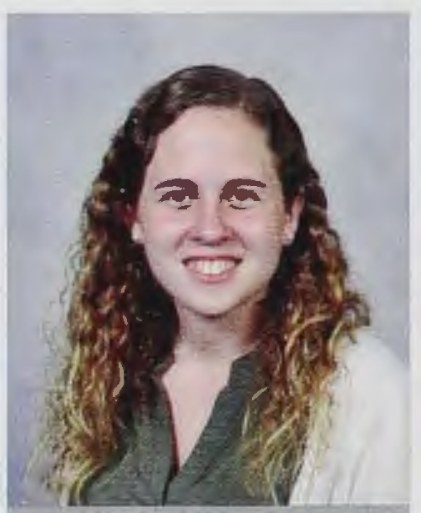

Jessica Schneider

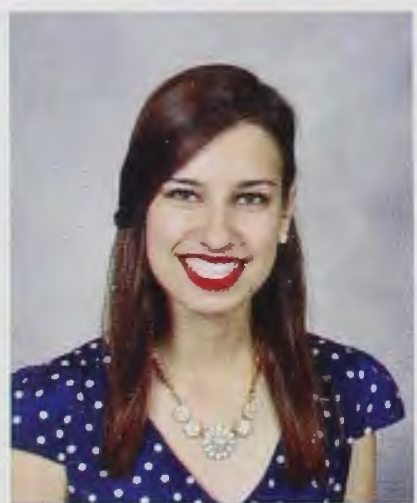

Angela Schweinitz

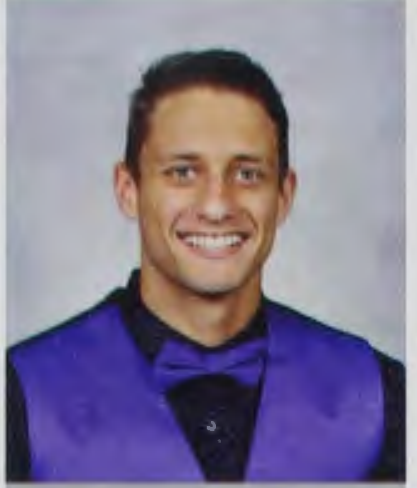

Rodrigo Reis

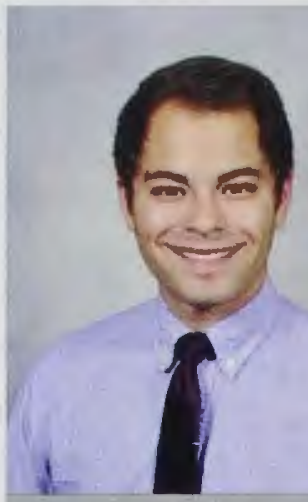

Matthew Rivera

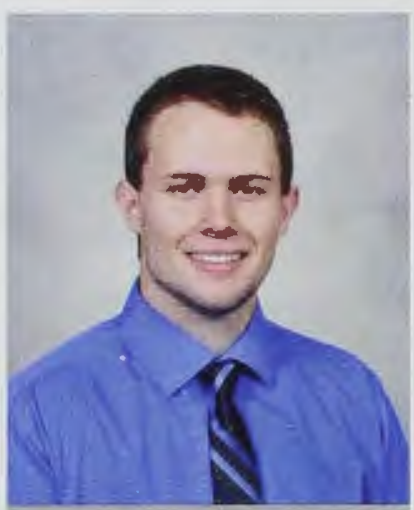

Austin Russell

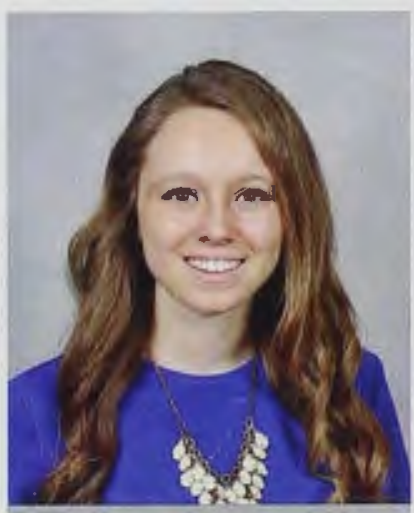

Lorrin Schoeneweis

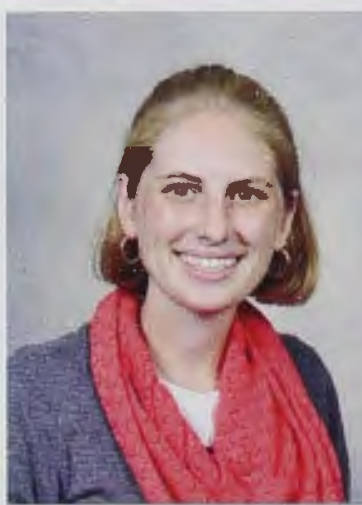

Erin Scott

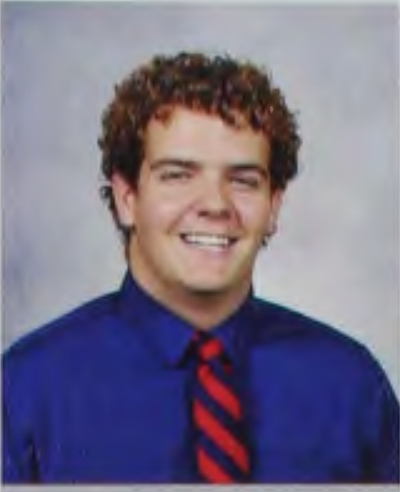

Robert Rhodes

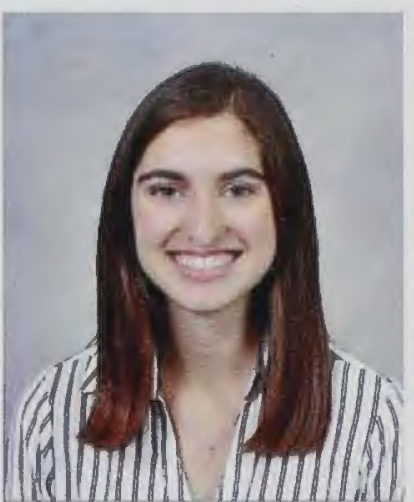

Victoria Rollins

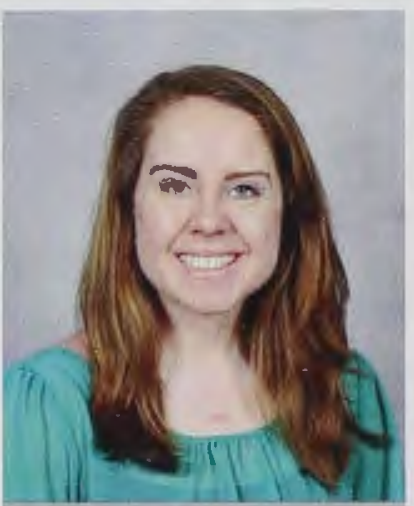

Jade Russell

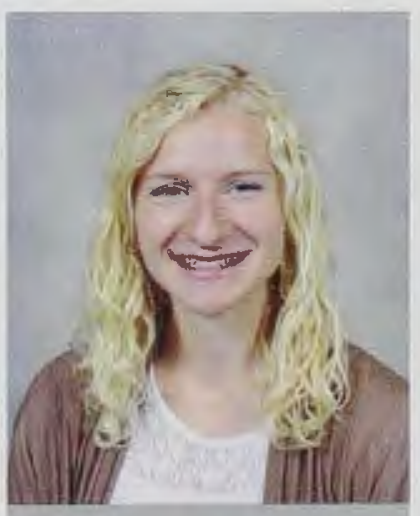

Anna Schoenwald

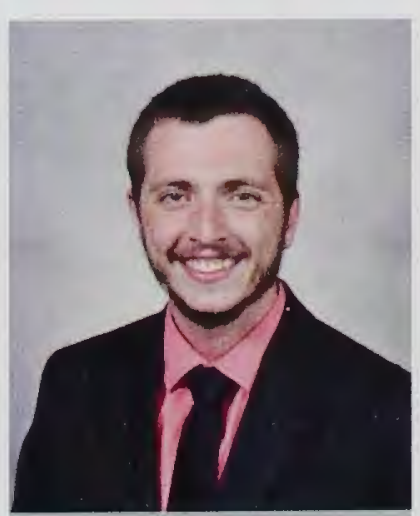

Timothy Shamburger

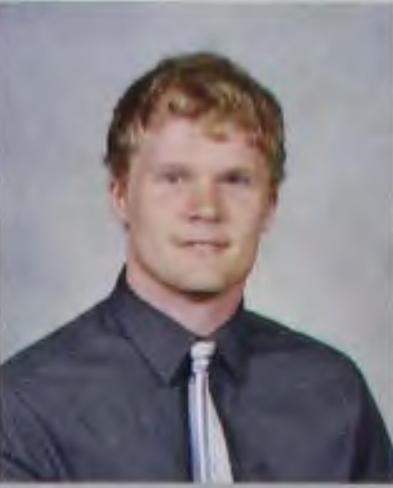

Elijah Richmond

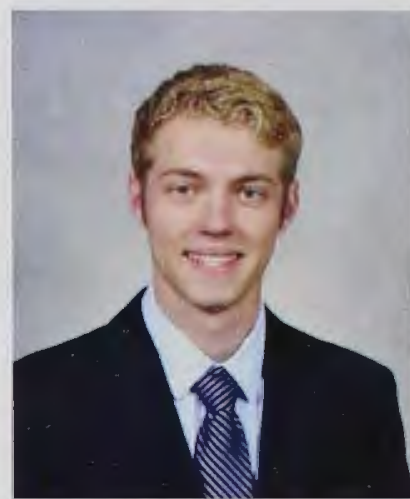

Nathaniel Ross

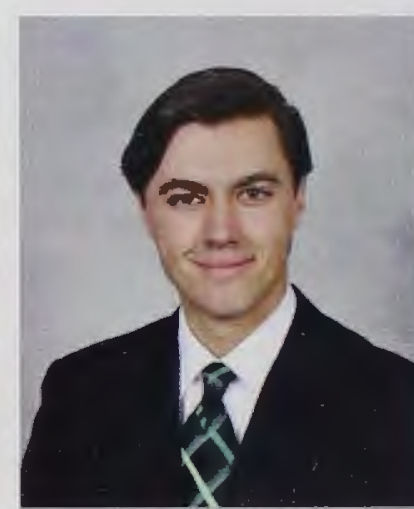

Joel Sabol

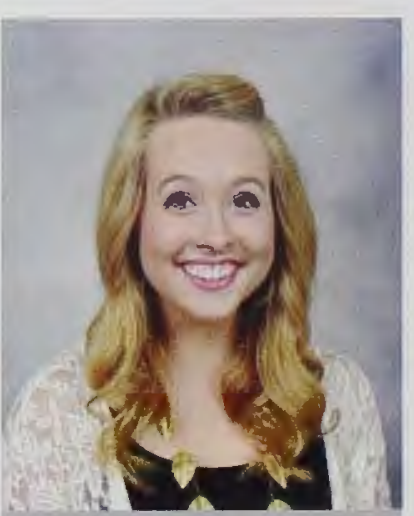

Emily Schumacher

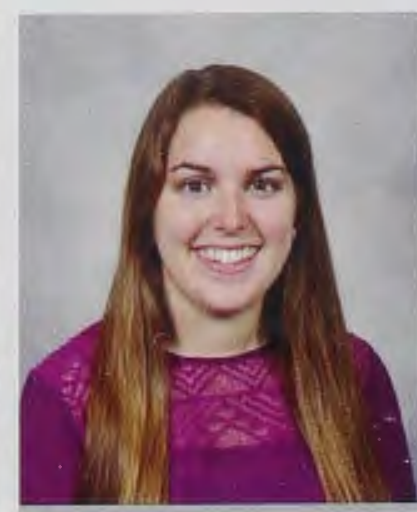

Muriel Shannon

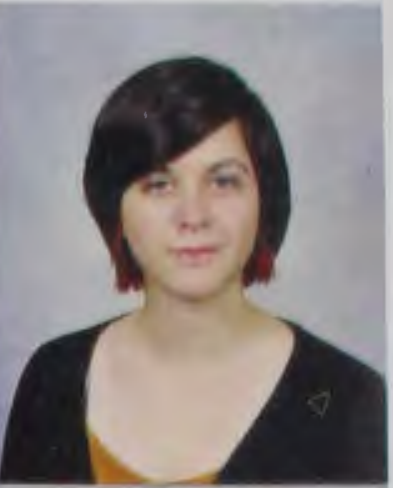

Jane Richmond

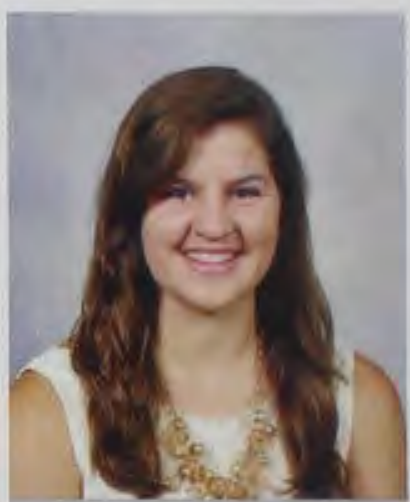

Christine Ruch

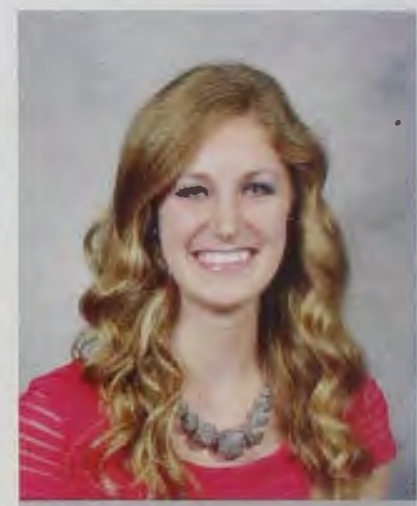

Lindsay Sanders

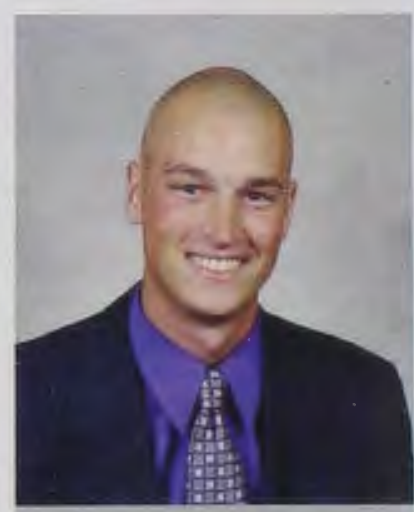

Caleb Schutt

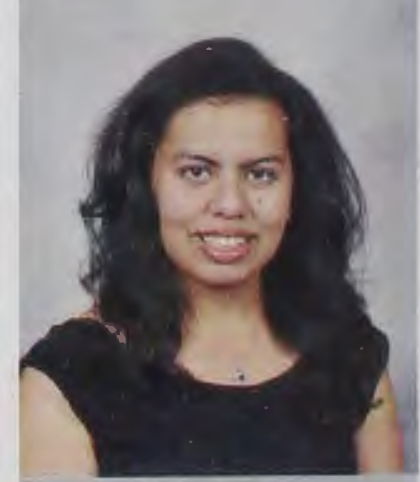

Gloria Shaw Ojeda 


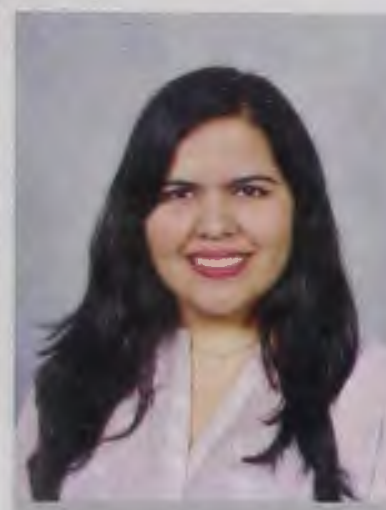

Myriam Shaw Ojeda

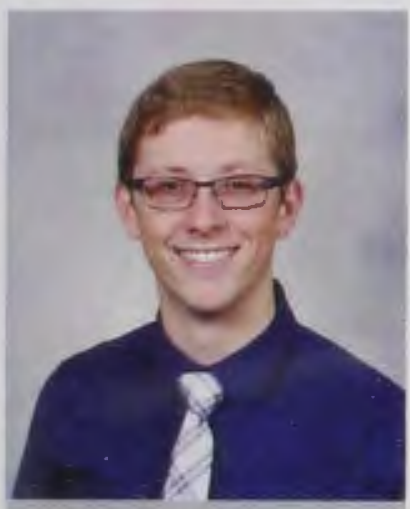

Connor Smith

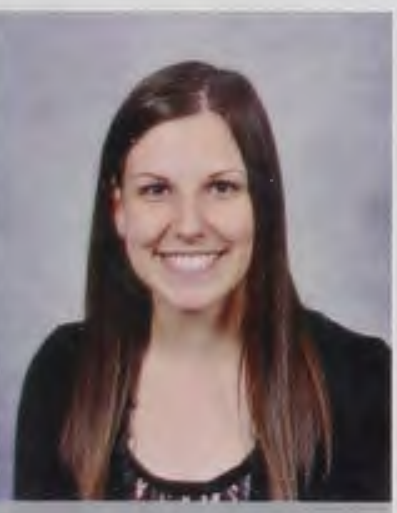

Brianna Sparks

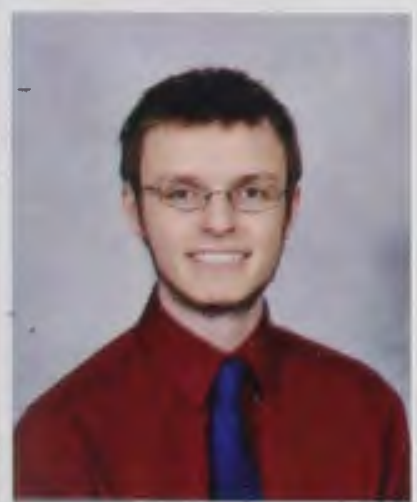

Grant Stannard

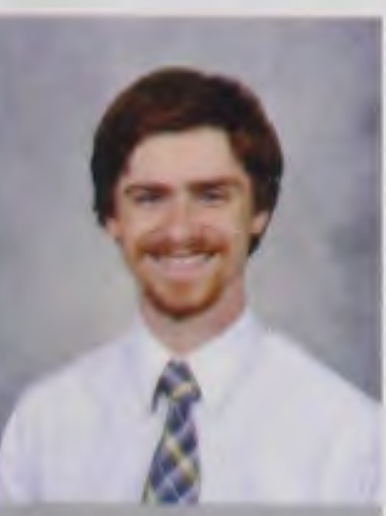

Phillip Stroh

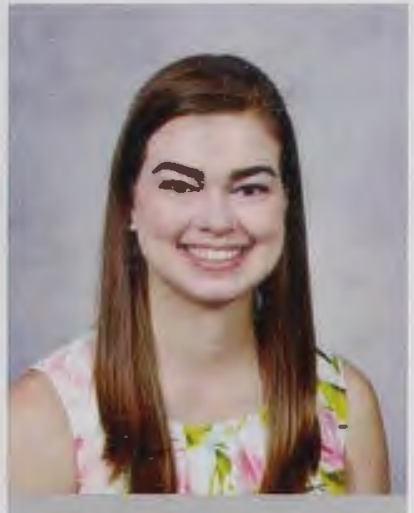

Emily Shinkle

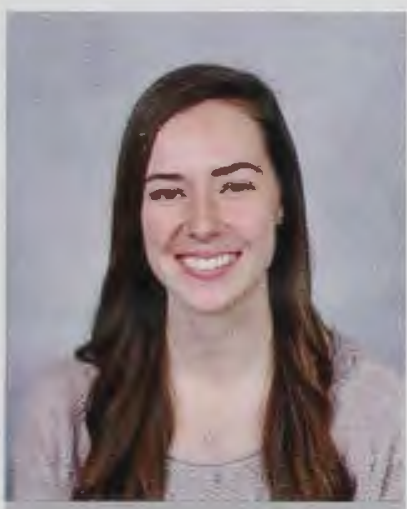

Emily Smith

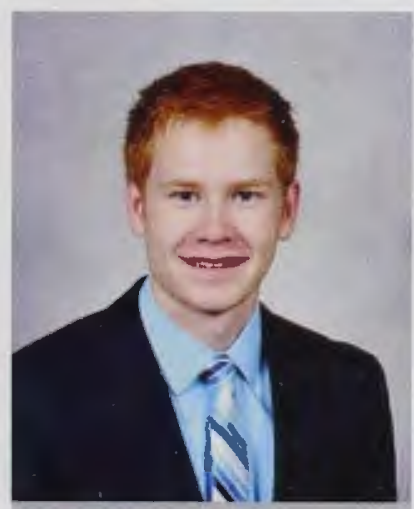

Andrew Spencer

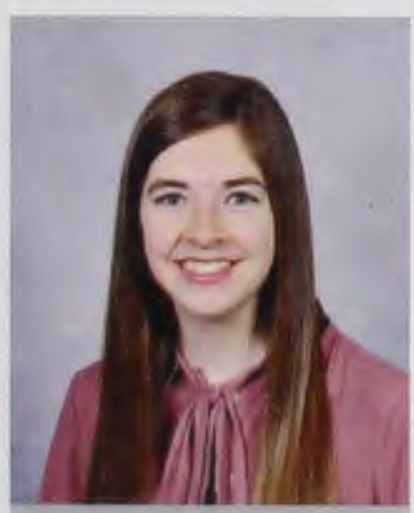

Hannah Steele

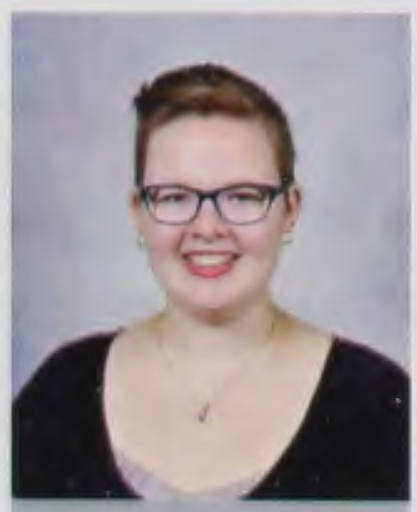

Julie Swanson

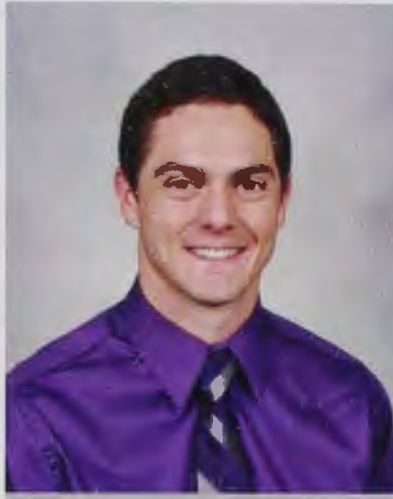

Nolan Shula

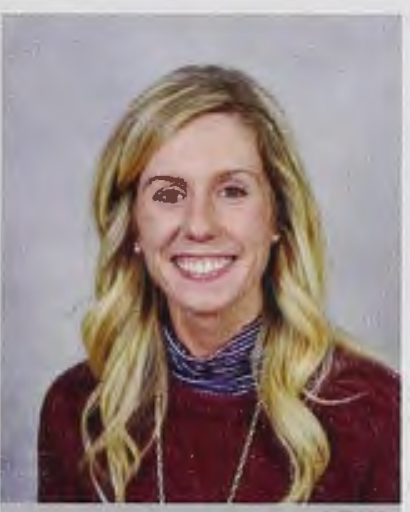

Kalie Smith

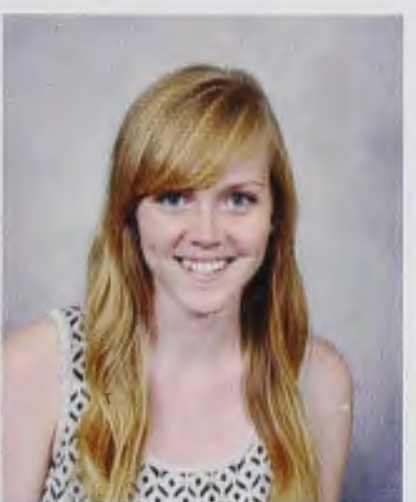

Victoria Spice

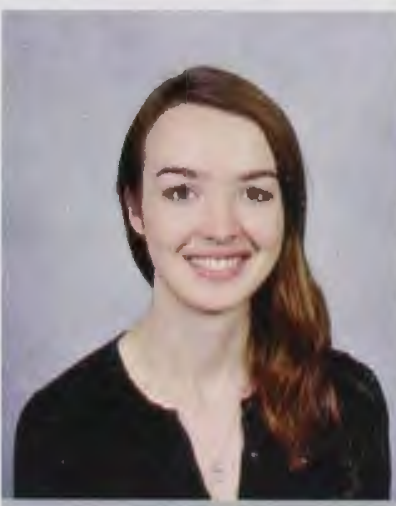

Taylor Stephens

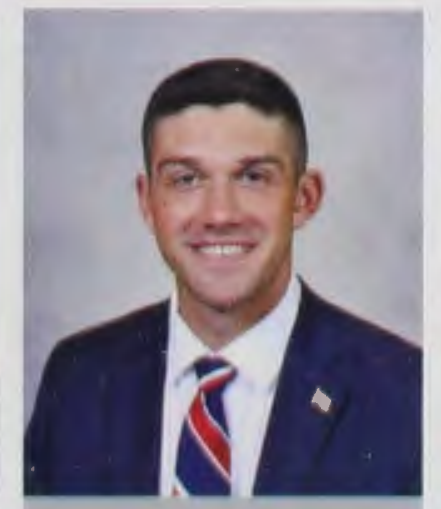

Ryan Swolsky

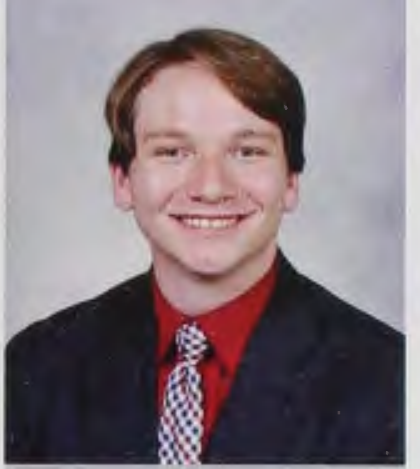

Jonathan Shumaker

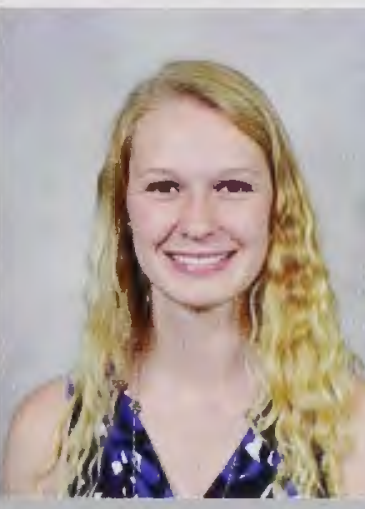

Valerie Sohn

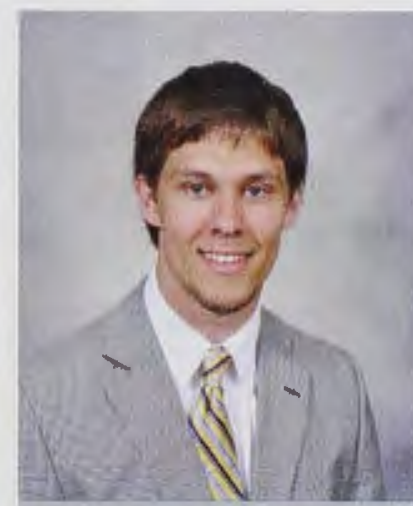

Samuel Spivey

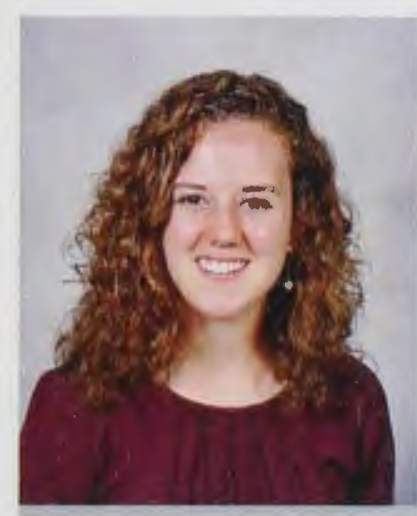

Madison Sternberg

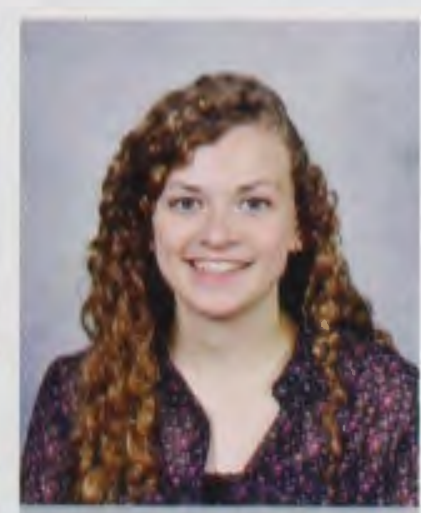

Alisha Symington

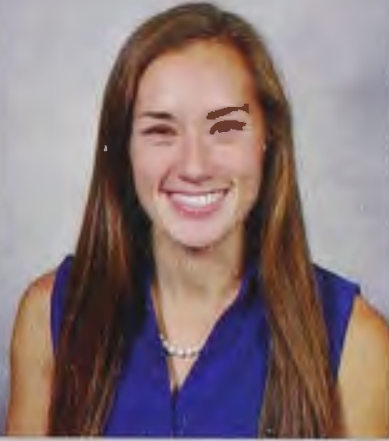

Kathryn Sill

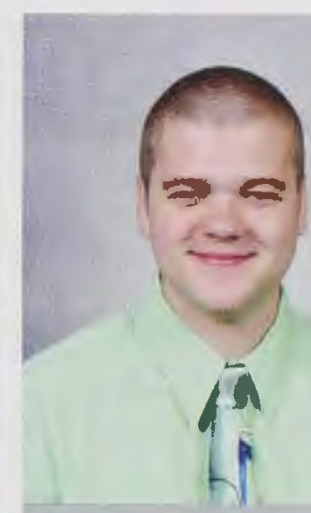

Brandon Solinger

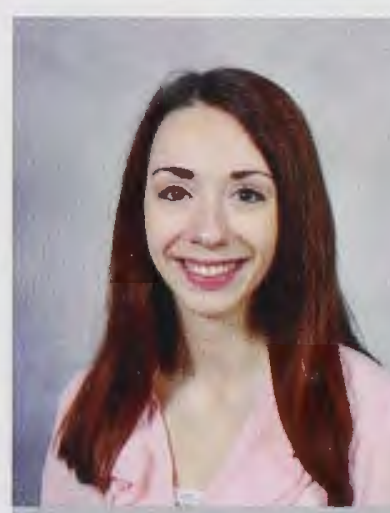

Amanda Stables

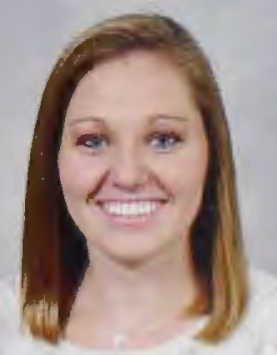

Maggie Stevenson

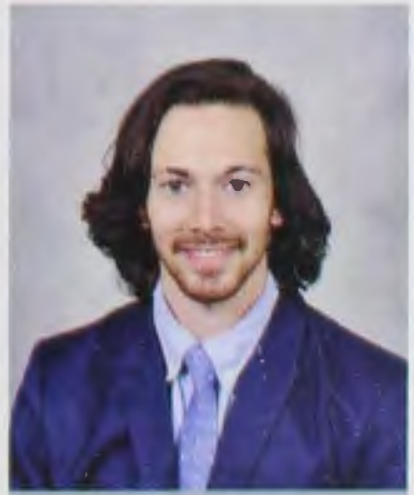

Mark Taggart-Perez

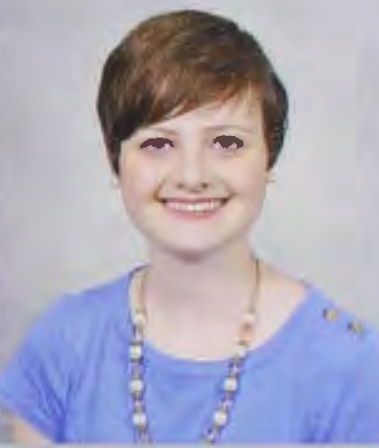

Elise Slone

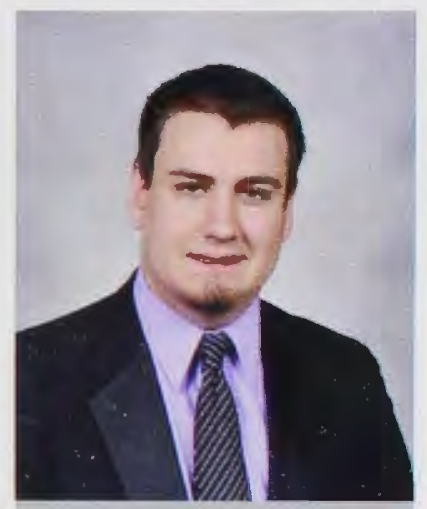

Joshua Sommers

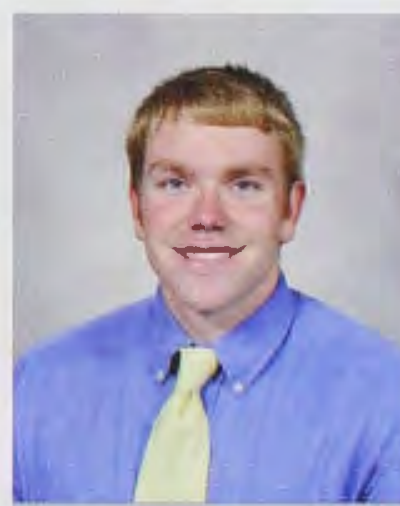

Samuel Stanaford

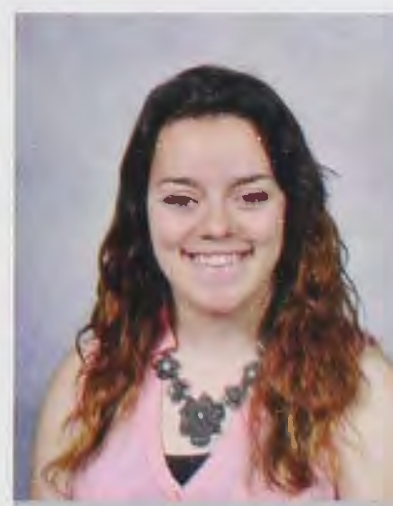

Melody Strayer

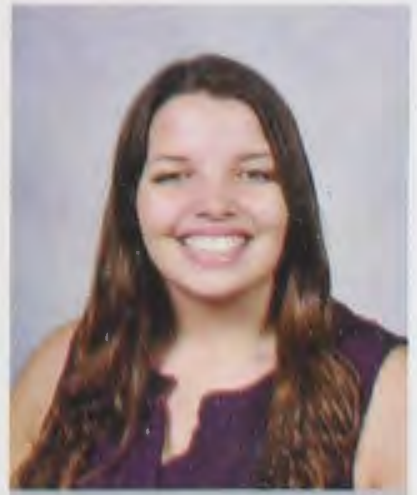

Jessica Tarr 


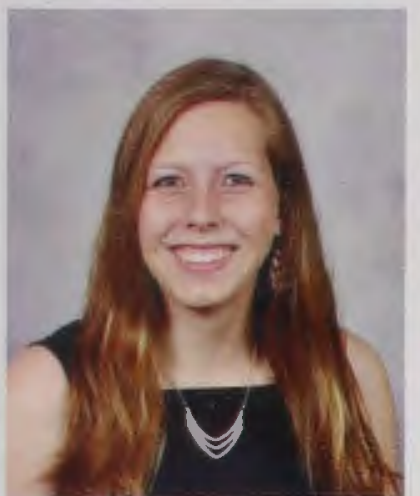

Maggie Weyandt

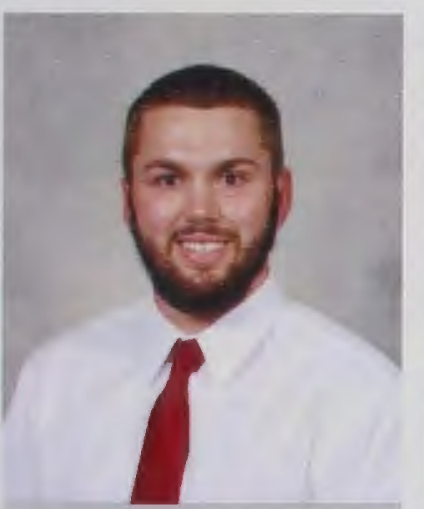

Cody Williams

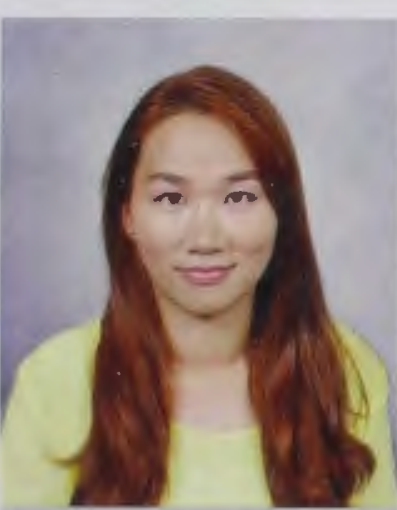

Linhan Xu

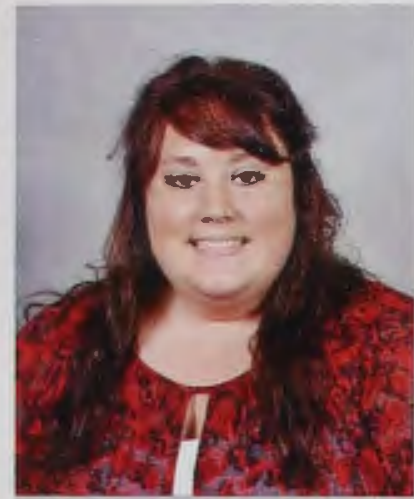

Kelly Whitmer

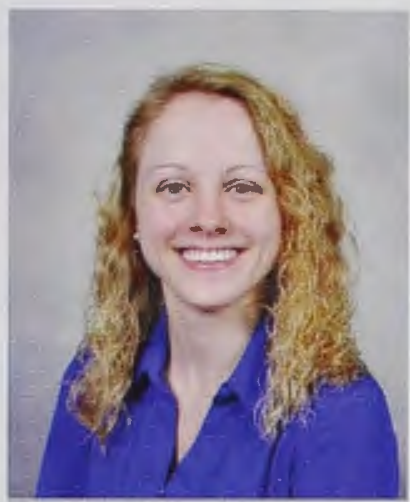

Emily Williams

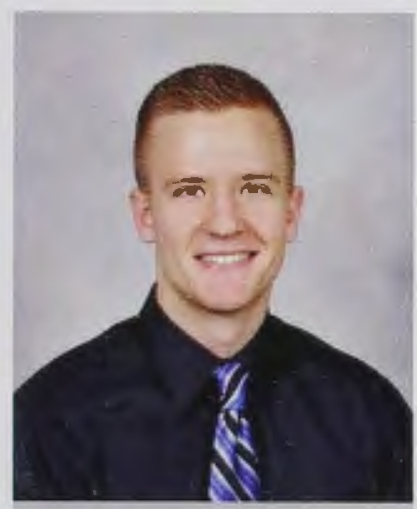

Brendan York

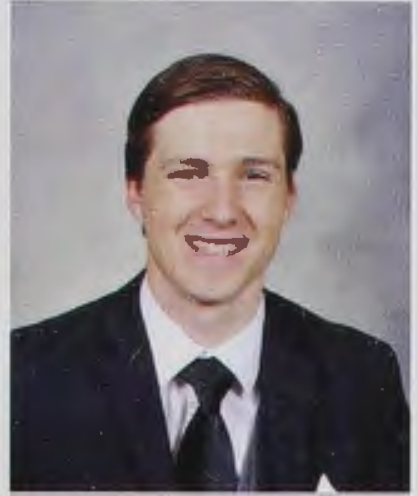

Joshua Wilbers

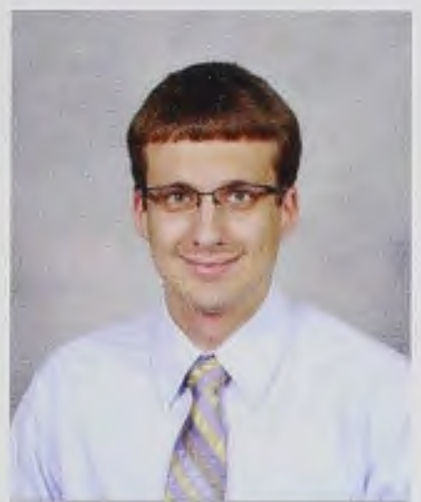

Joshua Wilson

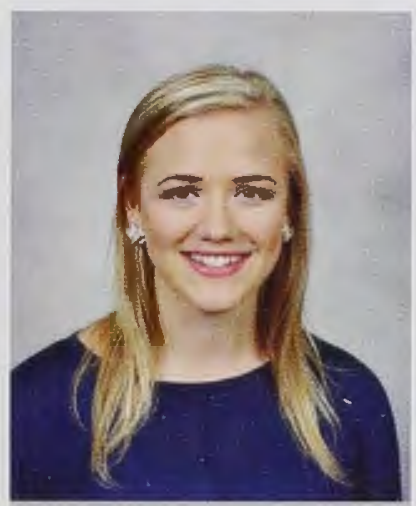

Julie Zavodney

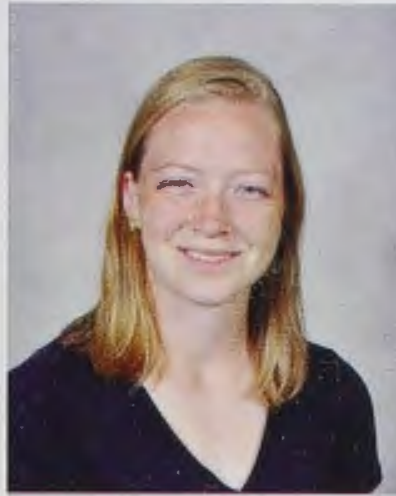

Taylor Wilcox

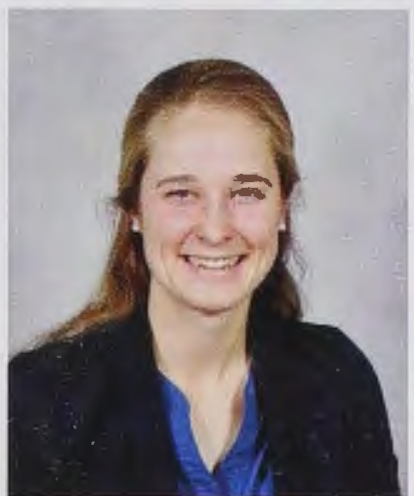

Katherine Wormald

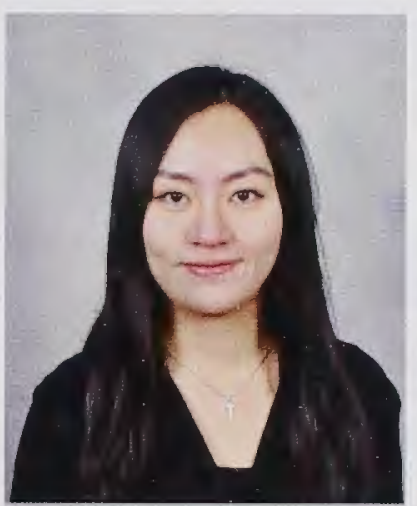

Zengmeizhi Zhong

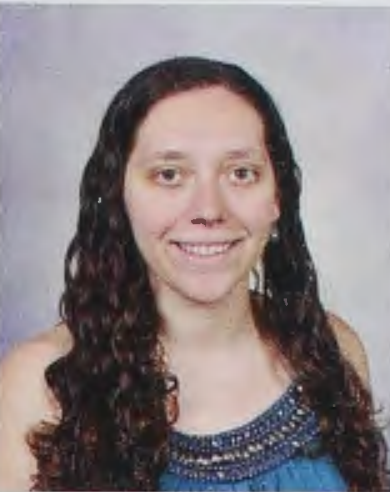

Hannah Willenbrink

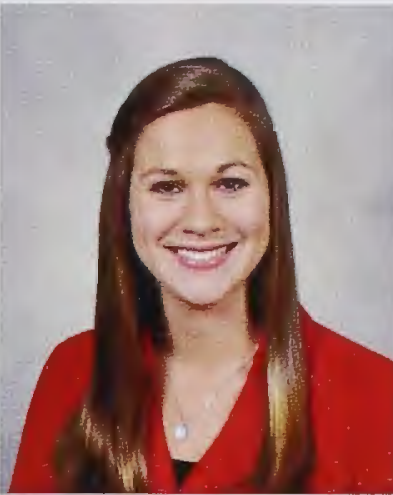

Jenna Wright

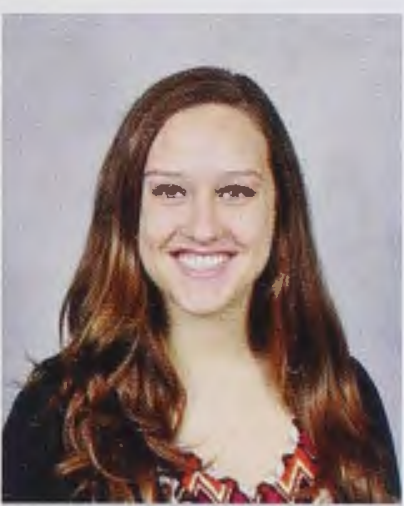

Emma Zuidema

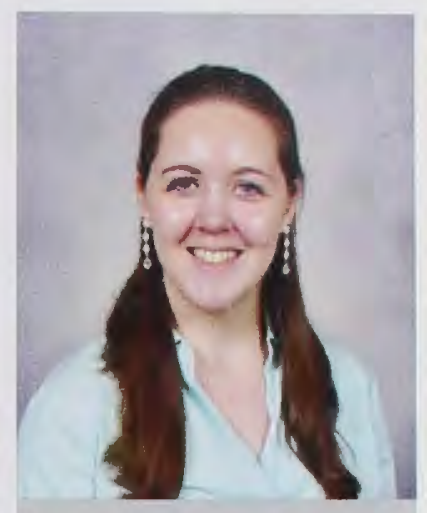

Allison Williams

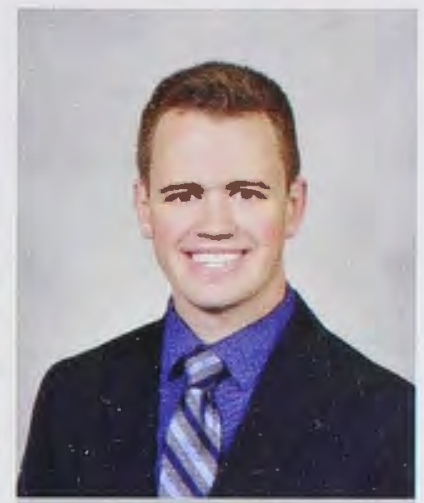

Mark Wynalda 


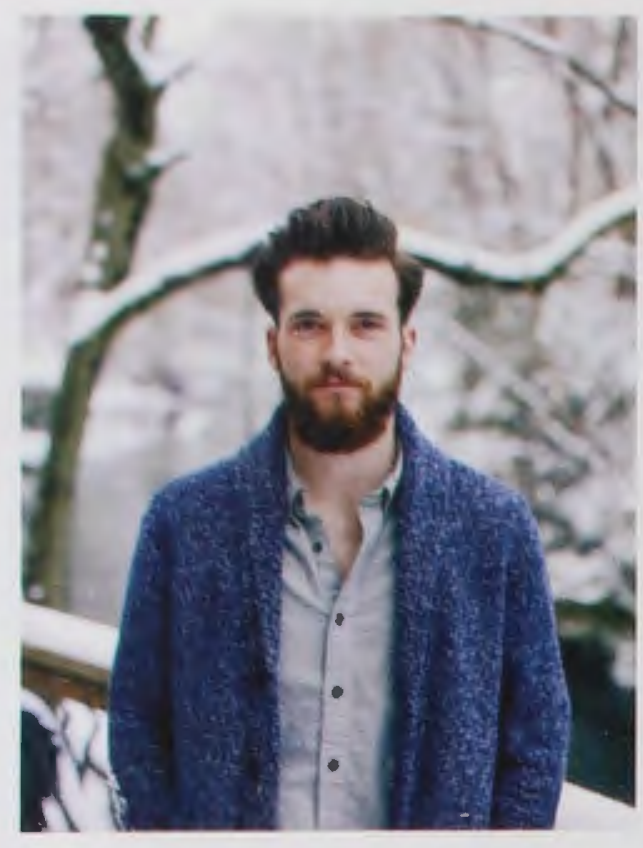

Peyton Green: Layout Editor

Peyton is a Junior graphic design major from Lewes, Delaware. He was responsible for the theme and page design of the book.

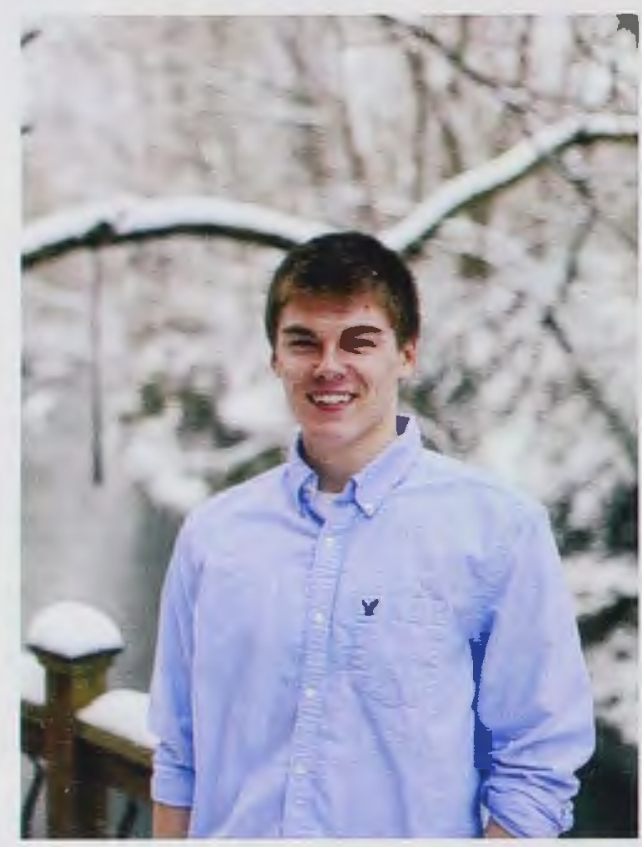

Nick Elder: Layout Assistant

Nick is a Sophomore graphic design major from Cincinnati, Ohio. He helped Peyton with designing annd creating the book this year.

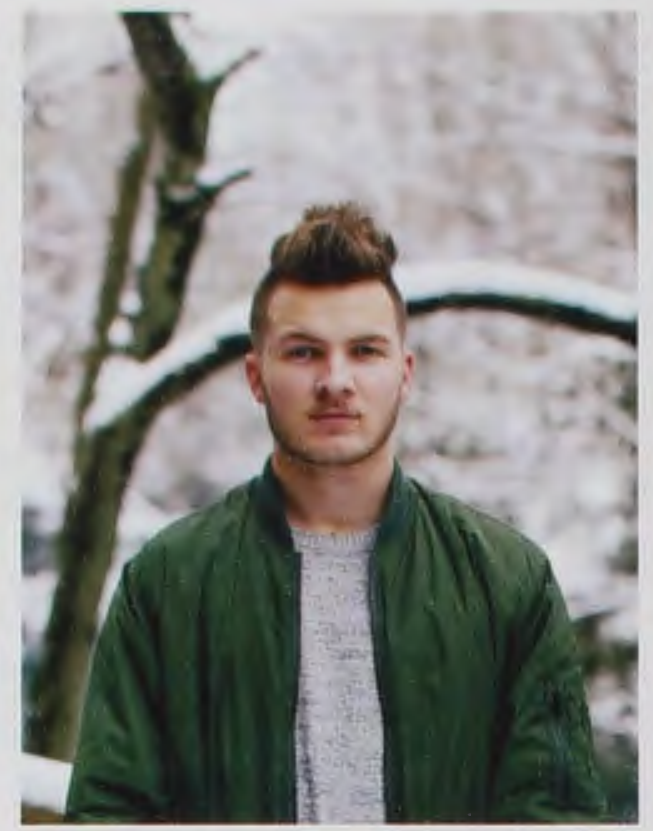

Justin Eisner: Photography Editor Justin is a Sophomore broadcasting and digital media major from Cary, North Carolina. He supervised the photographers and photo-editing for the book.

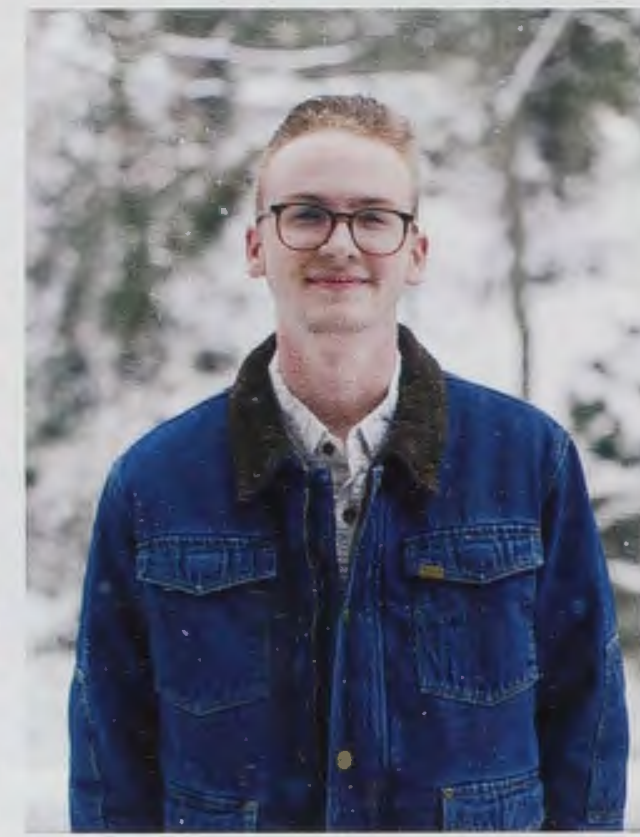

Cole Carpenter: Photographer

Cole is a Freshman broadcasting and digital media major from Dayton, Ohio. He was responsible for many of the photos in the book.

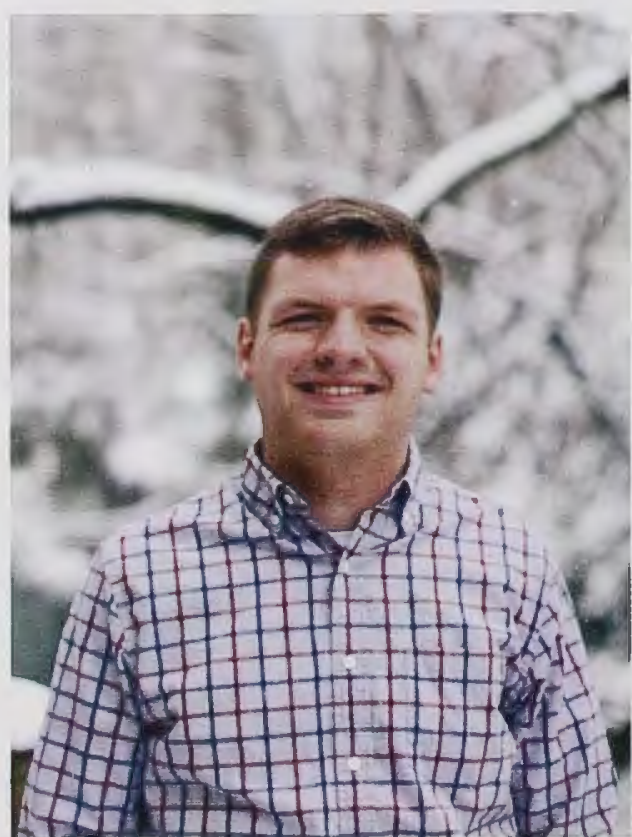

Ben Fawcett: Editor-in-Chief

Ben is a Senior business management major from Cedarville, Ohio. He was responsible for supervising staff and the overall production of the book
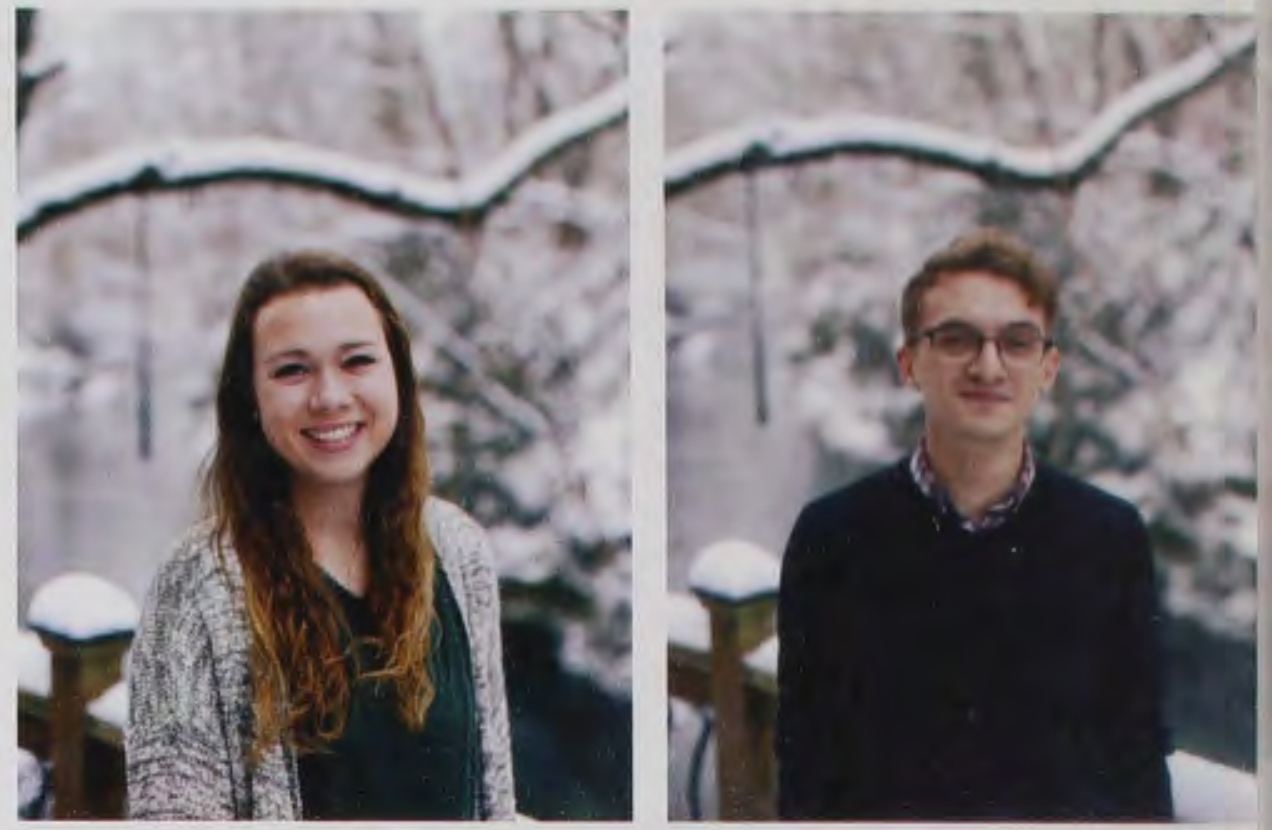

\section{Liz Coy: Administrative Assistant Marc Agee: Copy Editor}

Liz is a Sophomore communication and international studies major from Twinsburg, Ohio. She kept organized and maintained correspondence for the staff.

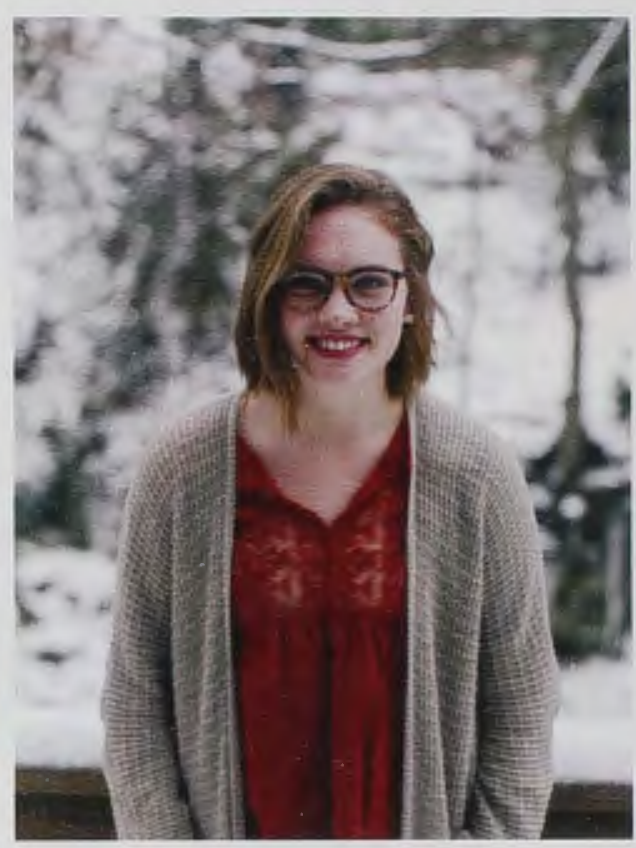

Hannah Ward: Photographer

Hannah is a Freshman industrial design major from Richmond, Michigan. She contributed many of the photos in the book.

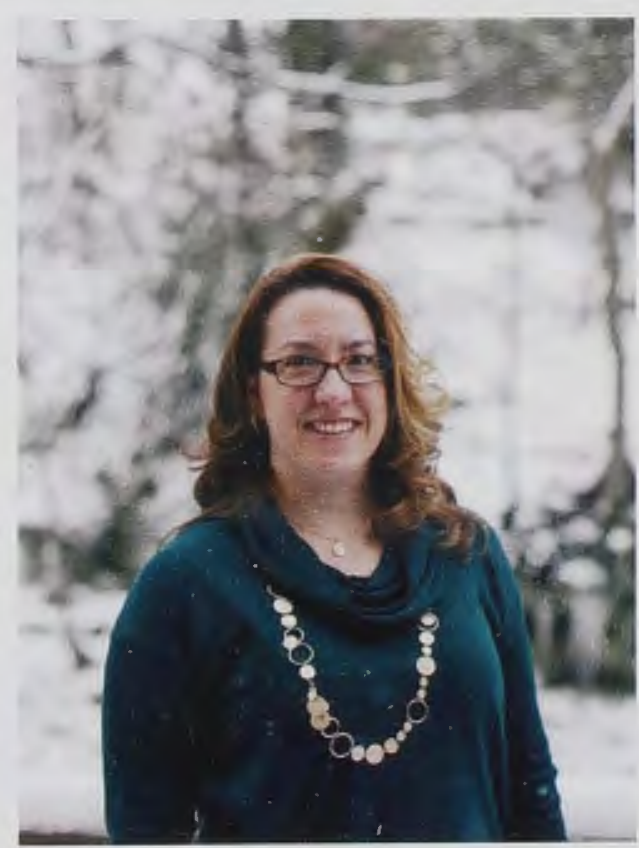

Cyndi Messer: Faculty Advisor

Cyndi Messer is an Associate Professor of English at Cedarville University. She provided resources and advice for the production of the book from Fairborn, Ohio. He was responsible for the writing of stories, captions, and quotes in the book.

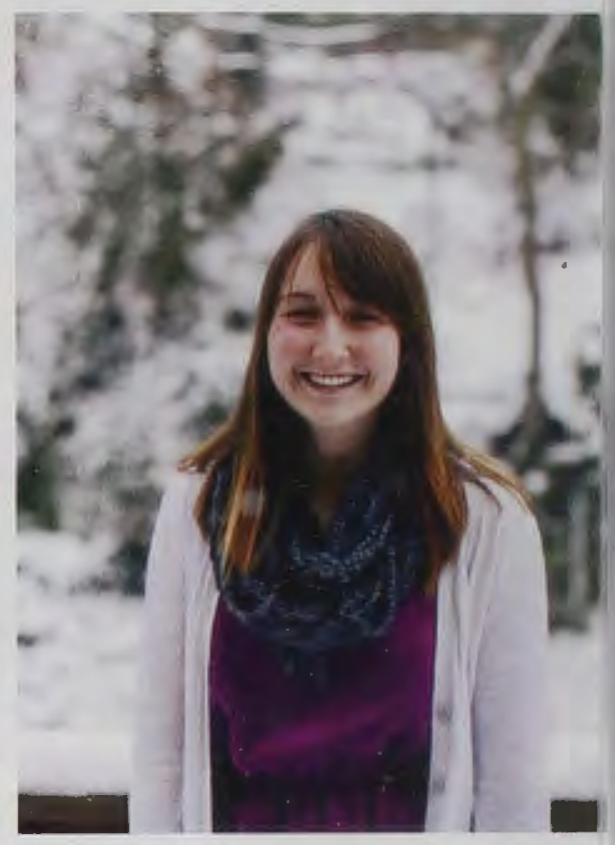

Jessica Westenberg: Copy Assist. Jessica is a Freshman early childhood and special education major from Laingsburg Michigan. She helped Marc with gatherir quotes and writing captions, and contributed stories for the book.
Marc is a Sophomore linguistics major 


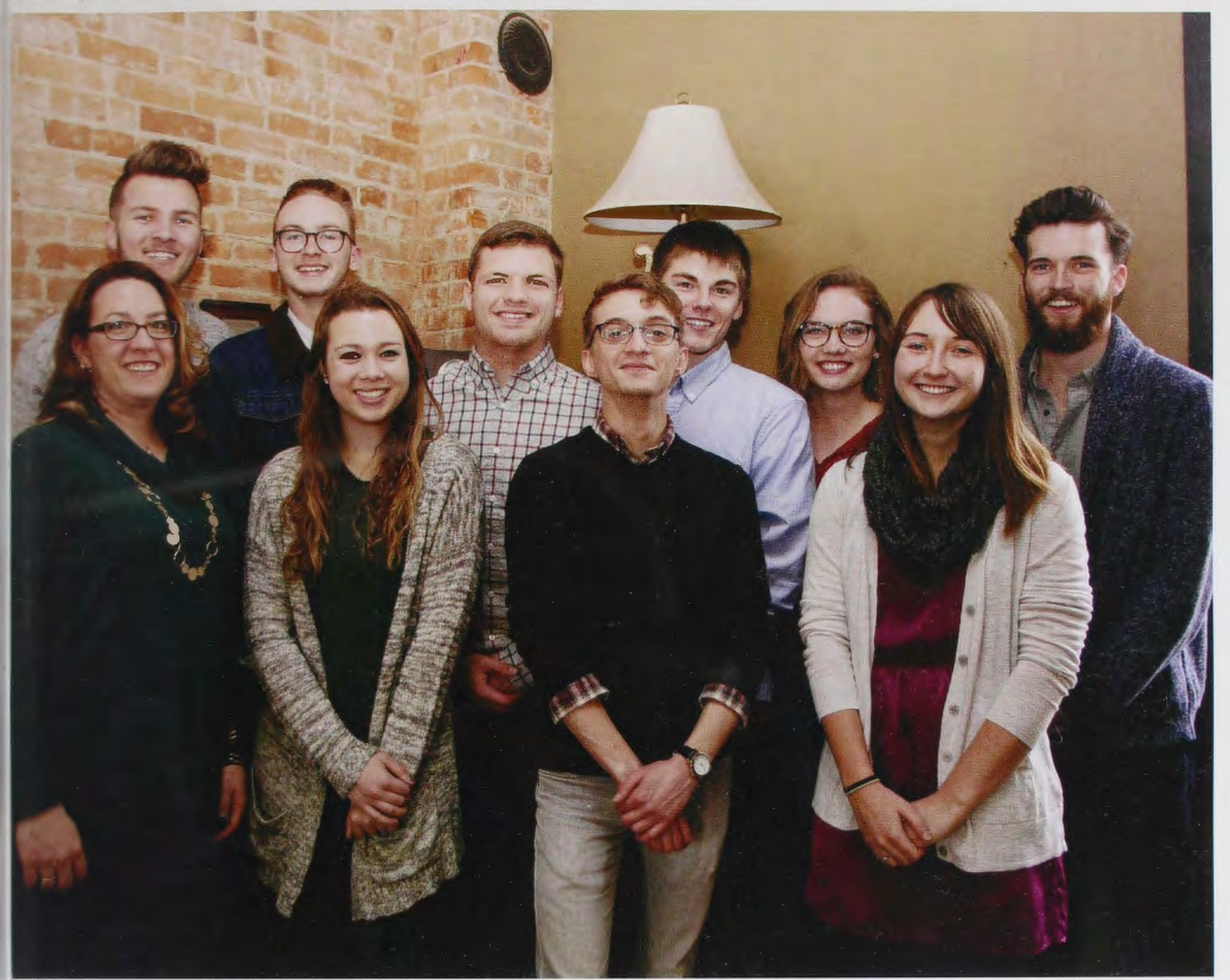

\section{Yearbook Staff}

The yearbook staff began as strangers who were all new to the yearbook. Over the year, they learned together what it took to design and create a yearbook. Through this shared learning, they came to be good friends, who looked forward to seeing each other at meetings, cared about each other's lives, had inside jokes, and helped each other with their work. Walks back to school from the office, getting coffee together, and wondering who was going to be the late one to any given meeting are some of their best memories together. 


\section{Colophon}

\section{Publisber}

The 2016 Miracle was published by Jostens, Inc. It was printed at their plant located in State College, PA. The Jostens representative was Donald Boyd, and the plant coordinator was Crystal Gault.

\section{Cover}

The cover is custom screen and is custom art designed by Peyton Green.

\section{Color}

The 2016 Miracle was printed with four color process ink.

\section{Design}

The 2016 Miracle was designed by Peyton Green.

\section{Theme}

The 2016 Miracle theme was a combined brainstorm of the editorial staff.

\section{Production}

The 2016 Miracle was produced on both HP and Apple Computers. Layouts were designed using Monarch by Jostens. Programs inside Monarch include Adobe InDesign and Adobe Photoshop.

\section{Typography}

The main font used in the book was Garamond Classic. The font was used in regular, bold, and italic styles. Size 10 was used for the captions, while size 12 was used for stories.

\section{Photography}

Photos were taken primarily by Justin Eisner with the help of his photography assistants, Cole Carpenter, and Hannah Ward. Several photos were taken by Scott Huck, photographer for Cedarville University. Jim McAdams provided senior, underclass, and faculty/staff portraits.

\section{Inquiries}

The Miracle Yearbook office may be contacted for additional information about this book's production at:

Miracle, Cedarville University

251 N. Main ST.

Cedarville, Ohio 45314

(937)766-4995

miracle@cedarville.edu 
As my time at Cedarville draws to a close, many transitions are occuring in my life. I'm getting married, I have a new job, and I'm moving to a new town. As all of the aspects of my life are changing, a constant thought that I have is, "What is it that I love about where I am now, and how can I manage to get that in my new circumstance?" For example, I love my job that I have had for 2 years...but why? At the end of the day, it's not making coffee that I love about my job as a barista. It's not the money that makes me love it. There is one aspect that remains.... and that is what I'm learning about.

People. That is the one aspect. People change everything. It's not all about where you live, how much you make, or the status you've achieved. It's about the people that are around you. Even when I've been faced with difficult, bad situations, it was the people that were around me that improved my circumstances.

So as I move from Cedarville, get married, and start a new job, I want to think more about the people I choose to be around more than the places I surround myself with. So I encourage you to consider who it is in your life that makes the places you're in worth being in. Surround yourself with people who will encourage you and want to be a positive influence in your life. Don't just let them pour into you. Pour into them. Take care of people, and they'll take care of you.

benjamin. 


For the Word of God and the Testimony of Jesus Christ 